\title{
Nonequilibrium phenomena in high Landau levels
}

\author{
I. A. Dmitriev \\ Institut für Theorie der Kondensierten Materie, Karlsruhe Institute of Technology, 76128 Karlsruhe, Germany \\ Institut für Nanotechnologie, Karlsruhe Institute of Technology, 76021 Karlsruhe, Germany and \\ loffe Physical Technical Institute, 194021 St. Petersburg, Russia
}

A. D. Mirlin

Institut für Nanotechnologie, Karlsruhe Institute of Technology, 76021 Karlsruhe, Germany Institut für Theorie der Kondensierten Materie, Karlsruhe Institute of Technology, 76128 Karlsruhe, Germany and Petersburg Nuclear Physics Institute, 188300 St. Petersburg, Russia

D. G. Polyakov

Institut für Nanotechnologie, Karlsruhe Institute of Technology, 76021 Karlsruhe, Germany

M. A. Zudov

School of Physics and Astronomy, University of Minnesota, Minneapolis, Minnesota 55455, USA

(Dated: October 31, 2018)

\begin{abstract}
Developments in the physics of $2 \mathrm{D}$ electron systems during the last decade revealed a new class of nonequilibrium phenomena in the presence of a moderately strong magnetic field. The hallmark of these phenomena is magnetoresistance oscillations generated by the external forces that drive the electron system out of equilibrium. The rich set of dramatic phenomena of this kind, discovered in high mobility semiconductor nanostructures, includes, in particular, microwave radiation-induced resistance oscillations and zero-resistance states, as well as Hall field-induced resistance oscillations and associated zero-differential resistance states. The experimental manifestations of these phenomena and the unified theoretical framework for describing them in terms of a quantum kinetic equation are reviewed. This survey also contains a thorough discussion of the magnetotransport properties of $2 \mathrm{D}$ electrons in the linear-response regime, as well as an outlook on future directions, including related nonequilibrium phenomena in other 2D electron systems.
\end{abstract}

Keywords: quantum Hall systems, nonequilibrium transport, two-dimensional electron gas, magnetooscillations

\section{Contents}

I. Introduction

II. Linear transport of $2 \mathrm{D}$ electrons in a moderately strong magnetic field

A. 2D electron gas: Types of disorder

1. Remote donors

2. Background impurities

3. Surface roughness

B. Magnetotransport beyond the Drude theory

1. Quantum magnetoresistance

2. Classical magnetoresistance

3. Classical magnetooscillations of the ac conductivity

C. Quantum magnetooscillations

1. Magnetooscillations of the density of states

2. Shubnikov-de Haas oscillations

3. Quantum magnetooscillations of the ac conductivity

4. Intersubband magnetooscillations

III. Microwave-induced resistance oscillations (MIRO)

A. MIRO: Experimental discovery and basic properties 16

B. Microscopic mechanisms of MIRO

1. Inelastic and displacement mechanisms

2. Saturation of the inelastic contribution at high radiation power and/or in a strong dc field

3. General approach: Quantum kinetic equation
4. Quadrupole and photovoltaic contributions to the photoconductivity

5. Classical mechanisms of MIRO

C. Related microwave-induced phenomena

1. Microwave-induced photovoltaic effects

2. Microwave-induced compressibility oscillations

3. Effects of a parallel magnetic field

4. MIRO in multisubband structures

5. MIRO in spatially modulated systems

6. Microwave-induced $B$-periodic oscillations

IV. Radiation-induced zero-resistance states (ZRS)

A. ZRS: Experimental discovery and basic properties

B. Instabilities of a nonequilibrium $2 \mathrm{D}$ electron gas and the emergence of domains in a strong magnetic field: Phenomenological approach

1. ZRS effective theory: Spontaneous symmetry breaking

2. Earlier results on related problems

C. Microscopic theory; determination of currents and fields in domains

D. Effective theories of the phase transition into ZRS and of ZRS dynamics

V. Hall field- and phonon-induced resistance oscillations (HIRO and PIRO)

A. HIRO: Experimental discovery and basic properties 38

B. Theory of nonlinear dc transport

C. Nonlinear resistivity: Inelastic effects

D. Zero-differential resistance states (ZdRS) 
E. PIRO: Experimental discovery and basic properties

F. PIRO in a strong Hall field

G. Microscopic theory of PIRO

VI. Transport in strong ac and dc fields

A. Fractional MIRO

1. Experiments at high microwave power levels

2. Multiphoton absorption via virtual states

3. Multiphoton absorption via sidebands

4. Multiphoton absorption via real states

B. Nonlinear mixing of MIRO and HIRO

1. Experiments on MIRO in a strong dc field

2. Frequency mixing for magnetooscillations

3. Fractional HIRO

4. AC field-periodic oscillations

VII. Outlook

A. Perspectives

1. Domain structure of ZRS and ZdRS

2. Giant photoresponse at the second harmonic of the cyclotron resonance

3. Nonequilibrium phenomena in separated Landau levels

4. Nonequilibrium transport at low temperature

5. Spin-related phenomena

6. Polarization dependence of MIRO

7. Nonequilibrium magnetotransport in the integer and fractional quantum Hall regime

B. Nonequilibrium magnetotransport phenomena in other $2 \mathrm{D}$ systems

1. Electrons on liquid helium

2. Graphene

VIII. Acknowledgments

References

\section{INTRODUCTION}

Fundamental research on two-dimensional electron gases (2DEGs), as well as on quantum wires and quantum dots made on the base of 2D structures, has largely determined the development of condensed matter physics in the last half-century (Beenakker and van Houten, 1991; Ferry and Goodnick, 1997). Continuous advances in the fabrication of semiconductor structures and the development of experimental techniques and theoretical approaches keep bringing up fascinating new areas of research. On the side of applications, these structures, serving as a basis for the planar semiconductor technology, are of enormous importance for modern nano- and optoelectronics (Alferov, 2001; Kroemer, 2001).

A large body of research findings concerning the character of electronic states, mechanisms of scattering, activated transport in the insulating regime, the cyclotron resonance, quantum magnetooscillations, and many other properties of a 2DEG has been summarized by Ando et al. (1982). The discovery of the integer and fractional quantum Hall (QH) effects in 1980-1982 largely shifted the focus of research towards the range of strong magnetic fields $B$ where the $\mathrm{QH}$ physics is observed (von Klitzing, 1986; Laughlin, 1999; Stormer, 1999; Tsui, 1999). More recently, the behavior of verylow-density 2DEGs at zero $B$ attracted a great deal of attention: the physics of these systems is governed by the interplay of quantum localization and strong interactions, which leads to a metal-insulator transition (Abrahams et al., 2001).

During the last two decades evidence has accumulated that the range of moderately strong $B$, where the cyclotron dynamics already sets in whereas the quantum localization effects and thus the Hall quantization are not developed yet, also reveals a rich variety of important physical phenomena. These include geometric resonances in periodically modulated structures (Weiss et al., 1989, 1991) and magnetoresistance due to quasiclassical memory effects and to interaction-induced quantum corrections to the conductivity [for a review, see (Dmitriev et al., 2008)].

A particularly prominent research arena was opened by the experimental discovery of the microwaveinduced resistance oscillations (MIRO) (Ye et al., 2001; Zudov et al., 2001a) and the zero-resistance states (ZRS) (Mani et al., 2002; Zudov et al., 2003). It became clear soon after these discoveries that a new field of nonequilibrium physics in high Landau levels (LLs) emerged. Most importantly, the observed phenomena demonstrated that the combined effect of the weak Landau quantization and the relatively weak microwave radiation can give rise to very strong changes of the transport properties of a 2DEG. These novel nonequilibrium phenomena revealed unexpected and conceptually interesting physics which has proven to be of interest in many areas of condensed matter physics. In addition to MIRO and ZRS, a number of other closely related phenomena have been observed, such as the Hall field-induced resistance oscillations (HIRO) (Yang et al., 2002a), the phonon-induced resistance oscillations (PIRO) (Zudov et al., 2001b), in particular, under nonequilibrium conditions created by a dc field (Zhang et al., 2008b), the microwaveinduced $B$-periodic oscillations in stronger magnetic fields (Kukushkin et al., 2004), the zero-differential resistance states (ZdRS) (Bykov et al., 2007; Zhang et al., 2008b), as well as the fractional MIRO (Dorozhkin, 2003; Willett et al., 2004; Zudov et al., 2003).

In the decade that has passed since the above discoveries, intensive experimental and theoretical work by many researchers has greatly advanced the understanding of the physics of nonequilibrium phenomena in high LLs. These advances have motivated us to write this review. While several short reviews are available (mainly written on a much earlier stage of development of the research field) (Dmitriev et al., 2008; Durst, 2006; Durst and Girvin, 2004; Fitzgerald, 2003; Lei, 2010; Lyapilin and Patrakov, 2004; Vitkalov, 2009), there is a clear need for a full-scale review article on the subject. In addition to summarizing past achievements, we also review important open questions as well as perspectives for further developments (including related nonequilibrium phenomena in other $2 \mathrm{D}$ systems). Particularly interesting in this respect is the recent discovery of photoinduced dissipationless transport in a $2 \mathrm{D}$ 
electron system on liquid He Konstantinov and Kono, 2009, 2010).

\section{LINEAR TRANSPORT OF 2D ELECTRONS IN A MODERATELY STRONG MAGNETIC FIELD}

We begin by reviewing the transport properties of a 2DEG in the linear-response regime, with emphasis on the effects that are qualitatively sensitive to the specific nature of disorder.

\section{A. 2D electron gas: Types of disorder}

Almost all work on the novel nonequilibrium magnetotransport phenomena at low magnetic field $B$ has relied on ultra-high mobility selectively doped GaAs/AlGaAs heterostructures - with the electron mobility $\mu$ at the level of $\mu \sim 10^{7} \mathrm{~cm}^{2} / \mathrm{V} \mathrm{s}$ (the mean free path $l \sim 10^{2} \mu \mathrm{m}$ ). Therefore, we focus below on the scattering mechanisms that are specific to these realizations of a 2DEG. Note that it is on the structures of this very design and this sample quality that the most spectacular advances in the study of the fractional QH effect have been made in the opposite limit of strong $B$. Typically, the 2DEG in high mobility structures is confined to a single quantum well (QW) in undoped ("pure") GaAs. In the single-interface setup, a triangular-shaped QW is created by the conduction band offset at the interface between AlGaAs and GaAs on one side and by the electric field produced by the charge of dopants, and of the 2DEG itself, on the other. The stability of the 2DEG charge confinement near the interface is supported by the chemical potential fixed by the overall distribution of charges. The double-interface design with a GaAs QW squeezed between AlGaAs layers is also frequently employed.

\section{Remote donors}

The key idea behind the suppression of impurity scattering in the heterostructures is a spatial separation of the layer to which the 2DEG is confined and the ionized donor impurities by an undoped "spacer" with a typical width $d \sim 10^{2} \mathrm{~nm}$. Since the first experiments on the modulation-doped heterostructures (Dingle et al., 1978), the optimization of the structure design has led to the growth of achieved mobilities from about $10^{4} \mathrm{~cm}^{2} / \mathrm{V} \mathrm{s}$ to the values in excess of $3 \times 10^{7} \mathrm{~cm}^{2} / \mathrm{V} \mathrm{s}$ (Pfeiffer and West, 2003; Umansky et al., 2009). Much of this progress has been related to the strong dependence of the momentum relaxation rate $1 / \tau=e / m \mu$ on $d$ for scattering off remote charged impurities (here and below $\hbar=1$ ):

$$
\frac{1}{\tau}=\frac{\pi n_{i}}{8 m\left(k_{F} d\right)^{3}},
$$

where $m$ is the electron mass, $k_{F}$ is the Fermi wavevector of the 2DEG, $n_{i}=\int d z c_{R}(z)(d /|z|)^{3}$, and $c_{R}(z)$ is the three-dimensional density of remote charged impurities located at $|z|>d$ ( $z$ is counted from the interface).

The transport scattering rate at zero temperature is obtained as an integral over the scattering angle $\phi$ :

$$
\frac{1}{\tau}=m \int_{0}^{2 \pi} \frac{d \phi}{2 \pi}(1-\cos \phi) W_{q=2 k_{F}} \sin (\phi / 2),
$$

where $W_{q}$ is the Fourier component of the correlation function of the screened impurity potential in the plane of the 2DEG

$$
W(r)=\langle V(0) V(\mathbf{r})\rangle, \quad \mathbf{r}=(x, y)
$$

at the transferred in-plane momentum $q$. In the limit $k_{F} d \gg 1$ (typically $k_{F} d \sim 10$ ), scattering is predominantly on small angles and Eq. (2) reduces to

$$
\frac{1}{\tau}=\frac{m}{k_{F}^{3}} \int_{0}^{\infty} \frac{d q}{2 \pi} q^{2} W_{q} .
$$

The function $W_{q}$ that describes remote charged impurities and upon substitution in Eq. (4) yields Eq. (10) is

$$
W_{q}=\left(\frac{\pi}{m}\right)^{2} n_{i} e^{-2 q d} .
$$

Equation (5) assumes that (i) $q^{-1}$ is much larger than the 2DEG thickness $w$ (typically $w \sim 10 \mathrm{~nm}$ ); (ii) the only source of screening of the impurity potential is the $2 \mathrm{DEG}$ and the dielectric function $\epsilon(q)$ is given by the $2 \mathrm{D}$ random phase approximation (RPA): $\epsilon(q)=\epsilon_{0}\left(1+2 / q a_{B}\right)$, where $\epsilon_{0}$ is the lattice dielectric constant at the interface and $a_{B}=\epsilon_{0} / m e^{2}$ is the Bohr radius; and (iii) $q^{-1}$ is much larger than $a_{B} \simeq 10 \mathrm{~nm}$. Conditions (i) and (iii) for the characteristic values of $q^{-1} \sim d$ are reasonably well satisfied in the high-mobility structures. The use of the RPA in (ii) relies on the smallness of the WignerSeitz parameter $r_{s}=\left(\pi n_{e} a_{B}^{2}\right)^{-1 / 2}$, where $n_{e}=k_{F}^{2} / 2 \pi$ is the density of the $2 \mathrm{DEG}$. For the typical value of $n_{e}=3 \times 10^{11} \mathrm{~cm}^{-2}, r_{s} \simeq 1$ and the conventional use of the RPA is only marginally justified. However, deviations from the RPA in Eq. (5) lead only to the parameter $m / \pi$ being replaced by the exact compressibility, so that at $r_{s} \sim 1 \mathrm{Eq}$. (5) remains valid up to a factor of order unity. We also note that fluctuations of the random potential created by remote impurities are accurately described by Gaussian statistics (i.e., higher cumulants can be neglected) in view of the typically large parameter $n_{i} d^{2} \gg 1$, independently of the value of $r_{s}$.

Note that the total scattering rate

$$
\frac{1}{\tau_{\mathrm{q}}}=m \int_{0}^{2 \pi} \frac{d \phi}{2 \pi} W_{q=2 k_{F}} \sin (\phi / 2)
$$

(which determines, e.g., the LL broadening induced by disorder, see Sec. II.C) is, in the case of remote impurities, much larger than $1 / \tau$. In the limit $k_{F} d \gg 1,1 / \tau_{\mathrm{q}}$ is rewritten, similarly to Eq. (4), as

$$
\frac{1}{\tau_{\mathrm{q}}}=\frac{2 m}{k_{F}} \int_{0}^{\infty} \frac{d q}{2 \pi} W_{q},
$$


which for $W_{q}$ from Eq. (5) gives $\tau / \tau_{\mathrm{q}}=\left(2 k_{F} d\right)^{2}$. The parametrically large, for $k_{F} d \gg 1$, difference between $\tau$ and $\tau_{\mathrm{q}}$ reflects a diffusive character of electron dynamics on the Fermi surface: the ratio $\tau / \tau_{\mathrm{q}}$ is a characteristic number of small-angle scattering events that is needed to change the direction of momentum by an angle of order $\pi$.

In an important case of "delta-doping," charged impurities are concentrated in a thin layer whose thickness is much smaller than $d$ (down to a few lattice constants), so that $c_{R}(z)$ can be approximated as $n_{i} \delta(z+d)$ with $n_{i}$ the sheet density of these impurities. The $\delta$-layer is grown either by directly adding impurities in AlGaAs or by doping into a short-period AlAs/GaAs superlattice embedded in the alloy (Friedland et al., 1996; Umansky et al., 2009). In the latter case, X-valley electrons in AlAs yield additional screening of disorder and a further reduction of $1 / \tau$. In modern high-mobility GaAs/AlGaAs structures, the growth sequence design typically includes one or two Si-doped $\delta$-layers (these may be followed by other layers with Si dopants which compensate for surface states at the boundary with the vacuum), which are the main supply of electrons to the 2DEG and are thought to be the main source of scattering in the 2DEG as far as the intentionally doped layers are concerned. A setup with two $\delta$-doped layers, one on each side of the $2 \mathrm{DEG}$, has the advantage that it yields larger $n_{e}$ for given $d$. The effect of the increase of $n_{e}$ on $1 / \tau$ is stronger than the enhancement of the strength of disorder. In the case of remote impurities with $n_{e}=n_{i}$, the time $\tau$ for a QW symmetrically doped from both sides is, according to Eq. (1), a factor of $\sqrt{2}$ larger than for the single-side doping. Moreover, the double-side doping has been instrumental in attaining the highest reported mobilities for which the scattering off remote impurities is likely to be of little importance, see Sec. II.A.2.

Equation (11) implies that charged impurities are randomly distributed in the $2 \mathrm{D}$ plane. This assumption appears to overestimate $1 / \tau$ (Buks et al., 1994a, b; Coleridge, 1997; Heiblum et al., 1984) and $1 / \tau_{\mathrm{q}}$ (Coleridge, 1991, 1997) measured in some heterostructures with $d \lesssim 50 \mathrm{~nm}$. The reason why the measured mobility is higher than expected was argued (Buks et al., 1994a, b) to be related to the fact that the Si-dopants in $\mathrm{AlGaAs}$ (in a range of $\mathrm{Al}$ content which includes the typical values of 30-40\%) may exist in two configurations: as shallow donors (which become positively charged after having supplied electrons for the 2DEG) and as DX centers (whose energies lie deeper in the forbidden gap and which can be negatively charged). Buks et al. (1994a, b) interpreted their experimental results to prove that the DX centers are negatively charged in the ground state and demonstrated that correlations in the spatial distribution of the positively and negatively charged impurities may lead to a strong reduction of the scattering rate, similar to the correlations considered earlier (Efros et al., 1990) for the case when not all donor impurities are ionized and some remain neutral. Control of the ratio of the impurity densities in the shallow and DX configurations indeed provided experimental evidence (Buks et al., 1994a b; Coleridge, 1997) for the correlations capable of substantially reducing $1 / \tau$ (by a factor of up to about 6 ), depending on the parameters of the technological process, and a similar behavior was also demonstrated in the measurements of $1 / \tau_{\mathrm{q}}$ (Coleridge, 1997; Shikler et al., 1997).

\section{Background impurities}

Electrostatics of the heterostructure (Stern, 1983) dictates that $n_{e}$ is lowered with increasing $d$ (Heiblum et al., 1984; Umansky et al., 1997). Details of the relation between $n_{e}$ and $d$ depend on the concrete doping design of the heterostructure; however, as long as the electric field in the spacer layer is mainly determined by the $2 \mathrm{DEG}$ charge, $n_{e}$ scales as $1 / d$, which gives for $n_{e}=n_{i}$, according to Eq. (1), $\mu \propto d^{5 / 2}$ (Lee et al., 1983). The growth of $\mu$ with increasing spacer thickness, observed at smaller $d$, typically stops in the ultra-high mobility samples at $d \simeq 60-70 \mathrm{~nm}$ (Umansky et al., 1997). This behavior is usually argued to be associated with a competition between scattering off remote charged impurities [Eq. (1)] and scattering off "background impurities" which are present in a small concentration in the spacer layer and also in the GaAs layer. Being distributed in close vicinity of the 2DEG, the background impurities can lead to large-angle scattering, thus giving the main contribution to $1 / \tau$ at sufficiently large $d$.

The momentum relaxation rate due to scattering off background impurities uniformly distributed with the three-dimensional densities $c_{B}^{<}$on one side of the interface and $c_{B}^{>}$on the other is obtained as

$$
\frac{1}{\tau}=\frac{\pi c_{B}}{m k_{F}^{3} a_{B}^{2}} \ln \left(\min \left\{k_{F}, w^{-1}\right\} a_{B}\right),
$$

where $c_{B}=c_{B}^{<}+c_{B}^{>}$and the corresponding correlation function $W_{q}$ for $q w \ll 1$ is given by

$$
W_{q}=\left(c_{B} / 2 q\right)\left[2 \pi e^{2} / \epsilon(q) q\right]^{2} .
$$

Equation (8) is accurate provided both $k_{F}^{-1}$ and $w$ are much smaller than $a_{B}$. In reality, both spatial scales in high-mobility GaAs/AlGaAs structures are about $a_{B} \simeq$ $10 \mathrm{~nm}$, so that $1 / \tau$ due to scattering off background impurities should then be sensitive to the exact shape of the electron density profile in the direction across the 2DEG plane. A comparison of Eqs. (1) and (8) shows that at $n_{i} / d^{3} \sim c_{B} / a_{B}^{2}$ (up to the logarithmic factor) the two mechanisms of scattering give equal contributions to the total scattering rate and at larger $d$ the scattering on background impurities dominates.

The dependence of $\mu$ on $n_{e}$ varied in situ by a controlled illumination in structures with $d$ larger than 85 and up to $200 \mathrm{~nm}$, exhibited - when fitted to a power law over about one decade in $n_{e}$ within the interval between $10^{10}$ and $2 \times 10^{11} \mathrm{~cm}^{-2}$ - the relation $\mu \propto n_{e}^{x}$ with $x$ in the 
range 0.6-0.7 (Pfeiffer et al., 1989; Shavegan et al., 1988; Umansky et al., 1997). Pfeiffer and West (2003) also emphasized that the power law with $x \simeq 0.7$ holds over two decades in $n_{e}$ for a series of samples with different $n_{e}$ for $\mu>10^{6} \mathrm{~cm}^{2} / \mathrm{V} \mathrm{s}$. These values of $x$ are substantially smaller than $3 / 2$ expected for scattering off remote impurities [Eq. (11)], which is commonly regarded as evidence pointing toward the dominant role of background impurities. Note that Eq. (8) for background impurities also predicts the exponent $3 / 2$ for sufficiently large $n_{e}$. However, in the low-density case $n_{e} \lesssim 10^{11} \mathrm{~cm}^{-2}$, the dependence of $\mu$ on $n_{e}$-according to the numerical calculations (Ando, 1982; Gold, 1989) using variational electron wavefunctions to treat the effect of a finite thickness of the 2DEG - is indeed substantially weaker for background impurities than $\mu \propto n_{e}^{3 / 2}$ [the results by Ando (1982) for background impurities are obtainable from those for remote impurities by putting $d \rightarrow 0$ ]. On the other hand, the numerical variational calculation by Walukiewicz et al. (1984) for $n_{e}>10^{11} \mathrm{~cm}^{-2}$ gave similar dependences of $\mu$ on $n_{e}$ for remote and background impurities.

A systematic experimental study Umansky et al., 1997) of the relative contributions of the scattering mechanisms was performed in single-interface heterostructures grown in a wide range of $d$ and $n_{i}$ and mobilities as high as $\mu \simeq 1.4 \times 10^{7} \mathrm{~cm}^{2} / \mathrm{Vs}$. By measuring the mobility at given $d$ and $n_{e}$ as a function of $n_{i}$ (see also Umansky et al., 2009), experimental evidence was presented that for $d$ larger than $70 \mathrm{~nm}$ the contribution of remote impurities to the total $1 / \tau$ was smaller (in the "best samples" - about 10-15\%) than that of background charges. These results indicate that $\mu$ in the presently available ultra-high mobility structures of various designs is likely to be mainly, or to a large extent, limited by background impurities.

In both Eqs. (1) and (8), $1 / \tau$ was obtained within the Born approximation. While this level of approximation is well justified for remote impurities, for $k_{F} a_{B} \sim 1$ background impurities that are within the distance $a_{B}$ from the $2 \mathrm{DEG}$ and give the main contribution (as far as the background charges are concerned) to the scattering rate are not really weak scatterers. The momentum relaxation time $\tau$ for the background impurities sitting effectively right at the interface is given simply by the time of flight $1 / v_{F} c_{B} a_{B}^{2}$ (where $v_{F}$ is the Fermi velocity) between two of them along the straight line. The presence of the strong scatterers at the interface will have important ramifications in the subsequent sections.

\section{Surface roughness}

Interface roughness is also an important source of disorder in high-mobility GaAs/AlGaAs structures and is argued (Markus et al., 1994; Saku et al., 1996; Umansky et al., 1997) to be probed by the anisotropy displayed by $\mu$ when the current is measured along different directions in the $2 \mathrm{D}$ plane. The anisotropy of interface roughness is viewed as an inherent property of the kinetics of growth of GaAs-based structures. Grown, as is usual in high-mobility structures, on the (001) plane, they exhibit atomic-scale terracing at the interface, with randomly shaped terraces being typically elongated in the [110] direction. Anisotropy of $\mu$ was reported to be in some high-mobility samples as large as 40\% (Markus et al., 1994; Saku et al., 1996; Tokura et al., 1992; Umansky et al., 1997), increasing with electron density. The experimental results indicate that the strength of interface roughness varies depending on the growth conditions: for two ultra-high mobility samples in Saku et al. (1996); Tokura et al. (1992), both having almost the same $\mu \simeq 10^{7} \mathrm{~cm}^{2} / \mathrm{V}$ s along the [110] direction, the anisotropy was about $40 \%$ in one of them and about $10 \%$ in the other. In the "best structures" studied by Umansky et al. (1997) the anisotropy was also reported to be reduced to $5-10 \%$. The experiments suggest that the characteristic correlation radius of the interface inhomogeneities ${ }^{1}$ that are relevant to the mobility is $10 \mathrm{~nm}$ (Markus et al., 1994; Saku et al., 1996; Tokura et al., 1992).

Modeling the interface as a hard wall, the problem of scattering by surface roughness reduces to that of a perturbation imposed on the boundary condition. Spatial fluctuations of the height of the surface $h(\mathbf{r})$ along the $z$ axis create then a $2 \mathrm{D}$ random potential whose correlation function at the transferred momentum $\mathbf{q}$ is (Ando et al., 1982; Prange and Nee, 1968)

$$
W_{\mathbf{q}}=F^{2}\langle h h\rangle_{\mathbf{q}},
$$

where $F=\int d z(\partial U / \partial z) \rho_{\mathrm{D}}(z)=\left.(\partial \chi / \partial z)^{2}\right|_{z=+0} / 2 m$, $U(z)$ is the unperturbed confining potential, $\rho_{\mathrm{D}}(z)$, nonzero for $z>0$, is the unperturbed electron density profile (normalized to unity) in the direction normal to the 2DEG plane, $\chi(z)=\rho_{\mathrm{D}}^{1 / 2}(z)$, and $\langle h h\rangle_{\mathbf{q}}$ is the correlation function of the surface corrugations. Equation (10) is valid for the characteristic amplitude of $h(\mathbf{r})$ much smaller than the 2DEG thickness. Estimates based on Eq. (10) (Ando, 1982; Markus et al., 1994; Saku et al., 1996; Tokura et al., 1992) show that the experimentally observed strong anisotropy can be explained in terms of scattering by interface roughness for realistic parameters of the latter. If the charge of the depletion layer can be neglected compared to the 2DEG charge, then $F \propto n_{e}$ and the dependence of the mobility on $n_{e}$, as it follows from Eq. (10), is $\mu \propto n_{e}^{-2}$, i.e., with increasing $n_{e}$ this

\footnotetext{
1 The issue of anisotropic interface roughness in ultra-high mobility GaAs/AlGaAs structures has attracted attention also in the context of cooperative effects in electron transport at half-filling of high LLs (Cooper et al., 2001; Willett et al., 2001). These experiments probed surface roughness at the interface with the vacuum and were focused on long-range statistically anisotropic fluctuations of the surface height with a much larger correlation radius on the scale of $1 \mu \mathrm{m}$.
} 
mechanism of scattering becomes stronger - in contrast to scattering off charged impurities. The calculation by Markus et al. (1994) shows that the $n_{e}^{-2}$ scaling of $\mu$ (for $F \propto n_{e}$ ) changes to $n_{e}^{-1 / 2}$ if scattering by interface roughness is predominately on small angles.

Although the term "interface roughness" commonly refers to the long-range fluctuations of $h(\mathbf{r})$, there are also inherent imperfections in the interface related to alloy disorder in the AlGaAs part of the heterostructure. These are correlated on the scale of the lattice constant $a_{0}$ and are described for $q a_{0} \ll 1$ by

$$
W_{q}=\Delta_{c}^{2} a_{0}^{3} I,
$$

where $\Delta_{c}$ is the energy scale of the fluctuations and the integral $I=\int d z \rho^{2}(z)$, with $\rho(z)$ being the electron density profile (normalized to unity) across the $2 \mathrm{DEG}$, is taken over the region where alloy scattering is present (Ando, 1982). The integral is estimated as $I \sim \xi^{5} / w^{6}$, where $\xi \sim a_{0}$ is the tunneling length under the barrier on the AlGaAs side of the interface and $w$ is the total thickness of the 2DEG. Although the values of $\Delta_{c}$ and $I$ can be calculated only numerically, early estimates (Ando, 1982) show that for $n_{e}=3 \times 10^{11} \mathrm{~cm}^{-2}$ and the Al fraction in the range $30-40 \%$ alloy scattering at the interface establishes the mobility limit at the level of about $10^{7} \mathrm{~cm}^{2} / \mathrm{V} \mathrm{s}$. This suggests that alloy scattering, commonly deemed irrelevant at the GaAs/AlGaAs interface, may in fact be important in the modern ultra-high mobility heterostructures.

\section{B. Magnetotransport beyond the Drude theory}

At the most basic level, dc transport in a magnetic field $B$ is described by the Drude formula according to which the resistivity tensor $\hat{\rho}(B)$ in two dimensions reads

$$
\hat{\rho}(B)=\frac{m}{e^{2} n_{e}}\left(\begin{array}{cc}
1 / \tau & \omega_{c} \\
-\omega_{c} & 1 / \tau
\end{array}\right),
$$

where $\omega_{c}=e B / m c$ is the cyclotron frequency. Equation (12) can also be derived within the formalism of the Boltzmann kinetic equation which has been very successful in explaining the magnetotransport properties of metals (Lifshitz et al., 1973; Pippard, 1989), the behavior of $\hat{\rho}(B)$ in which is known to depend in an essential way on the shape and topology of the Fermi surface. For an isotropic $2 \mathrm{DEG}$, which is a virtually perfect approximation for the 2DEG in GaAs as far as the band structure is concerned (note, however, the possible importance of anisotropic disorder discussed at the end of Sec. II.A), this theory predicts the simple Drude result (12). A striking feature of Eq. (12) is that the longitudinal resistivity $\rho_{x x}(B)=\rho_{\mathrm{D}} \equiv m / e^{2} n_{e} \tau$ is independent of $B$, so that the deviation

$$
\Delta \rho_{x x}=\rho_{x x}(B)-\rho_{x x}(0),
$$

termed a positive or negative magnetoresistance (MR), depending on the sign of $\Delta \rho_{x x}$, is exactly zero.

\section{Quantum magnetoresistance}

The MR in 2D systems has been studied extensivelyin the last three decades particularly in regard to $T$ dependent contributions coming from the influence of a magnetic field on the quantum corrections, small in the parameter $1 / k_{F} l \lesssim 1$, to the transport coefficients (Altshuler and Aronov, 1985). One quantum contribution to the MR is related to the suppression of the weak-localization correction to $\rho_{\mathrm{D}}$, which occurs in a "classically weak" (in the sense of $\omega_{c} \tau \ll 1$ ) magnetic field and results in a small sharp spike of $\rho_{x x}(B)$ at $B=0$. Another quantum contribution is due to the interaction between degenerate electrons multiply scattered by disorder. In the diffusive regime (where the temperature $T \ll 1 / \tau)$, the Coulomb interaction-induced MR obeys (for $r_{s} \ll 1$ and $\omega_{c} \tau \gg 1$ ) $\Delta \rho_{x x} / \rho_{\mathrm{D}}=$ $-\left(\omega_{c}^{2} \tau^{2} / \pi k_{F} l\right) \ln (1 / T \tau)$, irrespective of the nature of disorder (Altshuler and Aronov, 1985; Girvin et al., 1982; Houghton et al., 1982). The theory of this MR was extended to arbitrary $T \tau$ by Gornyi and Mirlin (2003, 2004). In the ballistic regime $(T \tau \gg 1)$, particularly relevant to high-mobility structures, the MR caused by electron-electron interactions depends in an essential way on the type of disorder, especially for $\omega_{c} \ll$ $T$, where it is strongly suppressed for smooth disorder. For $\omega_{c} \gg T$, if $1 / \tau$ is limited by smooth disorder, the Coulomb interaction-induced MR for $r_{s} \ll 1$ reads (Gornyi and Mirlin, 2003, 2004)

$$
\frac{\Delta \rho_{x x}}{\rho_{\mathrm{D}}}=-\frac{3 \zeta(3 / 2)}{32 \pi^{3 / 2}} \frac{1}{k_{F} l} \frac{\left(\omega_{c} \tau\right)^{2}}{(T \tau)^{1 / 2}}
$$

for $T \tau \ll r_{s}^{-2}$, while for $T \tau \gg r_{s}^{-2}$ it retains the same amplitude as in Eq. (14) but changes sign. If, by contrast, $1 / \tau$ is limited by white-noise disorder but the smooth component of disorder is still strong enough to produce a contribution to the momentum relaxation rate $1 / \tau_{L} \gg$ $\omega_{c}^{3} / k_{F}^{2} v_{F}^{2}$, the MR is enhanced compared to Eq. (14) by a factor $4\left(\tau_{L} / \tau\right)^{1 / 2}$ (Gornyi and Mirlin, 2004).

A crossover in the temperature behavior of the quadratic-in- $B$ MR between the diffusive and ballistic regimes was observed in the intermediate range of $T \tau \sim 1$ in moderate-mobility GaAs/AlGaAs structures by Li et al. (2003) [see also earlier works (Choi et al., 1986; Paalanen et al., 1983)], in close agreement with the theory for smooth disorder. The behavior of the MR in the crossover region was also studied in $\mathrm{Si} / \mathrm{SiGe}$ structures by Olshanetsky et al. (2005). The suppression of the MR as $T$ increases, observed by Galaktionov et al. (2006) in samples similar to those in (Li et al., 2003) in a wider range of $T \tau \gtrsim 1$, agrees favorably with the $T^{-1 / 2}$ scaling characteristic of the ballistic regime. The enhancement of the MR compared to Eq. (14), which can be largely attributed to the interplay of the short- and long-range components of disorder, was reported in a number of experiments (Bockhorn et al., 2011; Galaktionov et al., 2006; 
Olshanetsky et al., 2005), with a particularly strong enhancement observed by Bockhorn et al. (2011) in ultrahigh mobility GaAs/AlGaAs structures.

\section{Classical magnetoresistance}

Quite apart from the quantum MR, it was half a century ago that it was realized that long-range inhomogeneities can have a profound effect on magnetotransport; specifically, that even weak inhomogeneities with a spatial scale larger than the mean free path $l$ can yield a strong MR (Dreizin and Dykhne, 1973; Herring, 1960; Isichenko, 1992). In fact, even in macroscopically homogeneous (on the scale of $l$ ) electron systems, disorder with the correlation radius $d \gg k_{F}^{-1}$ can produce a strong quasiclassical MR (QCMR). The QCMR was discussed in a variety of systems: in the 3D Coulomb plasma (Murzin, 1984; Polvakov, 1986), in the 2D Lorentz-gas model (Baskin et al., 1978; Bobvlev et al., 1995), and in the 2DEG with smooth (Mirlin et al., 1999) and "mixed" (Mirlin et al., 2001; Polyakov et al., 2001) disorder. The essence of this phenomenon is that transport retains signatures of the underlying quasiclassical dynamics of electrons, which are not captured by the Boltzmann-Drude kinetic theory. Specifically, the QCMR is due to correlations in the otherwise random multiple scattering process at the points where the quasiclassical paths self-intersect. The strength of these "memory effects" - neglected in the collision-integral formalism - grows as a power of $d / l$. Below, we briefly discuss the results for the QCMR in a 2DEG.

The QCMR depends in an essential manner (even its sign does) on the correlation properties of disorder. Let us first review the case of Gaussian (in the sense of fluctuation statistics) disorder with the correlation radius $d \gg k_{F}^{-1}$. The MR is in fact nonzero Khveshchenko, 1996; Mirlin et al., 1998) within the collision-integral formalism because of the cyclotron bending of electron paths on the scale of $d$. This gives a small negative $\mathrm{MR}, \Delta \rho_{x x} / \rho_{\mathrm{D}} \sim-\left(d / R_{c}\right)^{2}$, where $R_{c}=v_{F} / \omega_{c}$ is the cyclotron radius. Correlations of diffusive paths at the points of self-intersection give rise to a much stronger positive MR: for $W_{q}$ from Eq. (5) and $\omega_{c} \tau \gg 1$ (Mirlin et al., 1999)

$$
\frac{\Delta \rho_{x x}}{\rho_{\mathrm{D}}}=\frac{2 \zeta(3 / 2)}{\pi}\left(\frac{d}{l}\right)^{3}\left(\omega_{c} \tau\right)^{9 / 2} .
$$

The characteristic momentum transfer $q$ in the scattering processes leading to Eq. (15) is $q \sim 1 / \Lambda \ll 1 / d$, where

$$
\Lambda=2 \pi^{1 / 2} v_{F} \tau /\left(\omega_{c} \tau\right)^{3 / 2}
$$

is the mean-square shift of the guiding center of a cyclotron orbit after one revolution. The ratio $\Delta \rho_{x x} / \rho_{\mathrm{D}}$ in Eq. (15) is much larger than that related to the effect of $B$ on the collision integral for $\omega_{c} \tau \gg(l / d)^{2 / 5}$ (note that $l / d \sim 10^{3}$ in ultra-high mobility structures) and becomes of order unity at $\omega_{c} \tau \sim(l / d)^{2 / 3}$. The latter condition corresponds to $\Lambda \sim d$. As $B$ is increased further, the strong positive MR is followed by an exponential falloff of $\rho_{x x}$ (Fogler et al., 1997):

$$
\ln \left(\rho_{x x} / \rho_{\mathrm{D}}\right) \sim-(d / \Lambda)^{2 / 3}
$$

which is due to the increasing adiabaticity of the electron dynamics and the related classical localization. The MR in Eq. (15), induced by rare $(\Lambda \gg d)$ self-intersections of diffusive trajectories, may be considered as a precursor of the strong (adiabatic) localization. Numerical simulations (Mirlin et al., 1999) confirm the strong QCMR for the case of smooth disorder. The QCMR of composite fermions scattered by effective random magnetic field was argued (Evers et al., 1999) to explain the dependence of $\rho_{x x}(B)$ around a half-filling of LLs (e.g., Smet, 1998).

Rare strong scatterers randomly distributed on top of smooth weak potential fluctuations can profoundly change the magnetotransport properties of a 2DEG (Mirlin et al., 2001; Polyakov et al., 2001). As discussed in Sec. II.A the combination of these two types of disorder - of comparable strength in what concerns the momentum relaxation rate - is likely to be an adequate description of a random potential in high mobility structures. The Drude formula (12) totally fails at $B \neq 0$ when only strong short-range scatterers are present: in an important early work by Baskin et al. (1978), a strong negative MR was shown in the classical 2D Lorentz-gas model. In this model, electrons are scattered by impenetrable hard disks ("voids"). In the limit of the density of the voids $n_{S} \rightarrow \infty$ with the momentum relaxation time $\tau_{S}$ held fixed, the model is exactly solvable (Bobylev et al., 1995) for the resistivity tensor $\hat{\rho}(B)$; in particular, $\rho_{x x} / \rho_{\mathrm{D}} \simeq 9 \pi / 8 \omega_{c} \tau_{S}$ for $\omega_{c} \tau_{S} \gg 1$ and the $\mathrm{MR}$ is exponentially small in the opposite limit. At finite $n_{S}$, the model shows a classical metal-insulator transition at a critical value of $R_{c} \sim n_{S}^{-1 / 2}$ (Baskin et al., 1978; Bobylev et al., 1995) and a quadratic MR in the low- $B$ limit (Cheianov et al., 2004).

When both types of disorder are present, the momentum relaxation rate at zero $B$ is $1 / \tau \simeq 1 / \tau_{L}+1 / \tau_{S}$, where $1 / \tau_{L, S}$ stands for the contributions of smooth disorder $(L)$ and strong scatterers $(S)$. Consider the relevant case of $\tau_{S} \ll \tau_{L}$ but smooth disorder being strong enough to produce $\Lambda$ [Eq. (16) with $\tau \rightarrow \tau_{L}$ ] larger than both correlation radii of disorder $d$ and $a$, where $a$ is the effective radius of strong scatterers. Then (Mirlin et al., 2001)

$$
\Delta \rho_{x x} / \rho_{\mathrm{D}}=-\left(\omega_{c} / \omega_{0}\right)^{2}
$$

for $\omega_{c} \ll \omega_{0}$, where

$$
\omega_{0}=\left(2 \pi n_{S}\right)^{1 / 2} v_{F}\left(2 \tau_{S}^{2} / \tau_{L} \tau_{0 S}\right)^{1 / 4},
$$

with $\tau_{0 S} \sim \tau_{S}$ being the total scattering time for strong scatterers. The ratio $\left(\omega_{c} / \omega_{0}\right)^{2}$ gives the fraction of the area "explored" twice because of the self-intersections of electron paths, which reduces the exploration rate and 

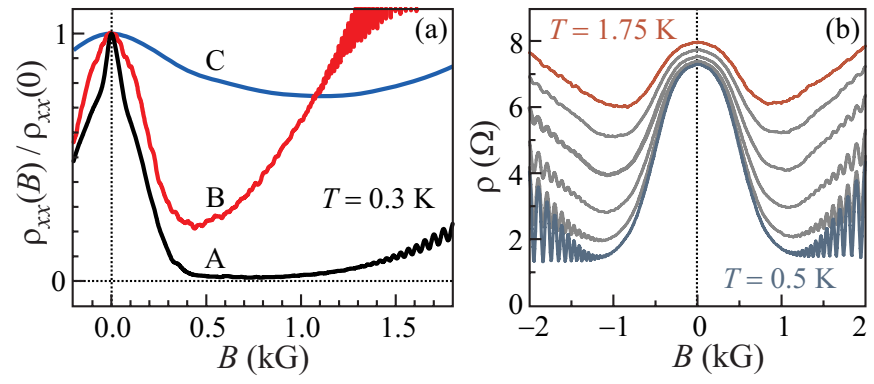

FIG. 1 (a) Magnetic-field dependence of the resistivity $\rho_{x x}$ (in units of $\rho_{x x}$ at $B=0$ ) of three different samples $\mathrm{A}, \mathrm{B}$, and $\mathrm{C}$ with the mobility of (A) $3 \times 10^{7} \mathrm{~cm}^{2} / \mathrm{V} \mathrm{s}$, (B) $1.2 \times 10^{7} \mathrm{~cm}^{2} / \mathrm{V} \mathrm{s}$, and $(\mathrm{C}) 8.6 \times 10^{6} \mathrm{~cm}^{2} / \mathrm{V} \mathrm{s}$ at $T=0.3 \mathrm{~K}$ and the electron density of (A) $2.9 \times 10^{11} \mathrm{~cm}^{-2}$ (B) $4.5 \times 10^{11} \mathrm{~cm}^{-2}$, and (C) $6 \times 10^{11} \mathrm{~cm}^{-2}$. Adapted from Dai et al. (2010). (b) Magneticfield dependence of the resistivity for different temperatures from 0.5 to $1.75 \mathrm{~K}$ in steps of $0.25 \mathrm{~K}$ in a sample with $\mu \simeq$ $5.4 \times 10^{6} \mathrm{~cm}^{2} / \mathrm{V} \mathrm{s}$ and $n_{e} \simeq 1.6 \times 10^{11} \mathrm{~cm}^{-2}$. Adapted from Hatke et al. (2012c).

leads to a longer time between collisions with different strong scatterers, hence the negative sign of the MR. This should be contrasted with the positive MR (15) for onescale smooth disorder, where the passages through the same area increase the scattering rate. For $\omega_{c} \gg \omega_{0}$, the scattering rate is strongly suppressed by the memory effects, which gives the $B^{-4}$ falloff as $B$ increases for $n_{S} R_{c}^{2} \gg 1$ (Mirlin et al., 2001; Polyakov et al., 2001),

$$
\rho_{x x} / \rho_{\mathrm{D}} \sim\left(\tau_{S} / \tau_{L}\right)\left(n_{S} R_{c}^{2}\right)^{2},
$$

and a plateau of $\rho_{x x}$ for $n_{S} R_{c}^{2} \ll 1$ with

$$
\rho_{x x} / \rho_{\mathrm{D}}=\tau_{S} / \tau_{L},
$$

i.e., the magnetic field "switches off" short-range scatterers which give the main contribution to $1 / \tau$ at $B=0$. This behavior of the QCMR has been observed in numerical simulations (Mirlin et al., 2001).

A negative MR with a pronounced resistance minimum at about $0.5 \mathrm{kG}$ was observed in early works on ultra-high mobility structures (Smet, 1997; Umansky et al., 1997). Recently, a strong negative MR in the same range of $B$ was reported in similar structures by Dai et al. (2010) and Hatke et al. (2011b, 2012c). In particular, in one of the samples studied by Dai et al. (2010), namely sample A in Fig. 17a, the resistivity was shown to decrease by a factor of about 50 between $B=0$ and $0.5 \mathrm{kG}$ and exhibit a wide plateau for larger $B$ before the onset of magnetooscillations, in qualitative agreement with the picture of the QCMR for the model of two-component disorder. With decreasing mobility (samples B and C in Fig. 1a), the plateau disappears but the negative MR persists. Analysis of the measured dependence of $\rho_{x x}(B)$ led Dai et al. (2010) to conclude that the observed phenomenon can be consistently described in terms of the QCMR theorized by Mirlin et al. (2001); Polyakov et al. (2001). A huge negative MR, with $\rho_{x x}(B)$ at the minimum of the $B$ dependence being at $T \simeq 0.3-0.5 \mathrm{~K}$ (for samples with different mobilities) several times smaller than at $B=0$, was reported by Hatke et al. (2012c) to be suppressed as temperature increases and disappear at about $2 \mathrm{~K}$. The $T$ dependence for one of the samples is shown in Fig. 1b. According to the analysis performed by Hatke et al. (2012c), the observed $T$ dependence is too strong to be described in terms of the theory of the quantum MR discussed in Sec. II.B.1.

The behavior of $\rho_{x x}(B)$ in the model of two-component disorder proves to be substantially more intricate when, as $B$ increases, $\Lambda$ becomes smaller than $2 d$ (in highmobility structures $d \gg a$ ) and scattering by the smooth random potential acquires the character of an adiabatic drift (Polyakov et al., 2001). Although the adiabaticity of scattering suppresses $\rho_{x x}$ when only smooth disorder is present [Eq. (17)], it can also give rise to the growth of $\rho_{x x}$ with increasing $B$ in the presence of shortrange disorder. This behavior can be most clearly seen in the "hydrodynamic" model where short-range disorder is characterized by white-noise correlations and the problem is mapped onto that of advection-diffusion transport (Isichenko, 1992). In the limit of large $B$, the hydrodynamic model predicts $\rho_{x x} / \rho_{\mathrm{D}} \sim\left(\tau_{S}^{2} v_{F} d / \tau_{L} R_{c}^{2}\right)^{5 / 13}$. Note that for realistic parameters the full-fledged QCMR in the adiabatic regime competes with the effects of Landau quantization (Sec. II.C). A growth of $\rho_{x x}$ with increasing $B$ in the experiments by Dai et al. (2010) and Hatke et al. (2011b) was observed in a range of $B$ well before the onset of oscillations [see also the earlier results (e.g., Galaktionov et al., 2006) on a positive MR observed at sufficiently high $T$ in moderate-mobility structures]. The huge negative MR observed by Dai et al. (2010) and Hatke et al. (2011b, 2012c), as well as the growth of $\rho_{x x}$ at higher $B$, warrants further study. In this respect, it is interesting to note that, experimentally, there appears to be a connection between the huge negative MR and a pronounced anomaly in the photoresponse; for details see Sec. VII.A.2

\section{Classical magnetooscillations of the ac conductivity}

Within the Boltzmann kinetic theory, the dissipative diagonal ac conductivity $\sigma(\omega)=\sigma_{+}(\omega)+\sigma_{-}(\omega)$, which determines the absorption rate for linearly polarized electromagnetic waves, is given by the Drude formula

$$
\sigma_{\mathrm{D}, \pm}(\omega)=\frac{e^{2} n_{e} \tau}{2 m} \frac{1}{1+\left(\omega_{c} \pm \omega\right)^{2} \tau^{2}} .
$$

The quasiclassical correlations in the dynamics of multiple scattering, which lead to the MR discussed in Sec. II.B.2, can also strongly modify the ac response of a 2 DEG at $B \neq 0$. In particular, they yield periodic-in$\omega / \omega_{c}$ oscillations in $\sigma(\omega)$ which are of essentially classical nature. For the model of two-component disorder (Sec. II.B.2), the oscillatory classical correction $\Delta \sigma^{(c)}(\omega)=\sigma^{(c)}(\omega)-\sigma_{\mathrm{D}}(\omega)$ in units of the Drude conductivity $\sigma_{\mathrm{D}}(\omega)$ takes, in the large- $B$ limit, the form of 
sharp resonant features at the harmonics of the cyclotron resonance (CR) at $\omega=M \omega_{c}$ with $|M|=1,2, \ldots$, the amplitude of which is given (Dmitriev et al., 2004) by

$$
\frac{\Delta \sigma^{(c)}\left(M \omega_{c}\right)}{\sigma_{\mathrm{D}}\left(M \omega_{c}\right)}=-\frac{a\left(\omega_{c} \tau_{L}\right)^{1 / 2}}{\sqrt{3 \pi}|M| \Lambda}
$$

where $a=1 / n_{S} v_{F} \tau_{S}$, and the width $\Gamma_{M}=3 M^{2} / 2 \tau_{L}+$ $1 / \tau_{0 S}$. Equation (23) is valid for $\Gamma_{M} \ll \omega_{c}$. In the opposite limit of strongly damped oscillations, $\Delta \sigma^{(c)}(\omega)$ reads (Dmitriev et al., 2004)

$$
\frac{\Delta \sigma^{(c)}(\omega)}{\sigma_{\mathrm{D}}(\omega)}=-\frac{a}{\pi^{1 / 2} \Lambda} \cos \frac{2 \pi \omega}{\omega_{c}} \exp \left(-\frac{3 \pi \omega^{2}}{\omega_{c}^{3} \tau_{L}}\right) .
$$

The behavior of $\sigma^{(c)}(\omega)$ is illustrated in Fig. 2, where also the quantum oscillations related to Landau quantization (Sec. II.C.3), are shown. One sees that the classical oscillations can be much more pronounced than the quantum ones for $\tau_{\mathrm{q}} \ll \tau_{L}$ (which is the case in high-mobility structures). Resonant features, of similar origin, at the harmonics of the CR were also discussed for the case of a random antidot array (Polvakov et al., 2002). The CR in the opposite limit of a smooth random potential was considered by Fogler and Shklovskii (1998) who found, in particular, a sharp jump, as $B$ is increased, in the CR broadening from the Drude width $1 / \tau$ to the width of separated LLs $\left(\omega_{c} / \tau\right)^{1 / 2}$ at the crossover to the adiabatic localization regime.

It is also worth noting that the ac conductivity exhibits magnetooscillations that are not associated with either the long-time correlations in disorder-induced scattering [Eq. (24)] or Landau quantization but are entirely due to electron-electron interactions (Sedrakvan and Raikh, 2008). Namely, they are related to the sensitivity of screening of the impurity potential to the cyclotron bending of quasiclassical electron paths. However, in contrast to the oscillations in Fig. 2, the period of the interactioninduced oscillations in $1 / \omega_{c}$ is much larger (by a factor of the order of $\left.\left(\epsilon_{F} /|\omega|\right)^{1 / 2} \gg 1\right)$ than $1 /|\omega|$, so that for given $\omega$ these oscillations might only develop for much smaller $B$ and much smaller $T$.

\section{Quantum magnetooscillations}

In our discussion so far, we neglected the effects related to Landau quantization by assuming that the electron $\operatorname{DOS} \nu(\varepsilon)$ at $\varepsilon$ equal to the Fermi energy $\varepsilon_{F}$ does not depend on $B$. In this section, we review the oscillatory behavior of the transport coefficients of a $2 \mathrm{DEG}$ with varying $\varepsilon_{F} / \omega_{c}$ and $\omega / \omega_{c}$-which arises from Landau quantization and is collectively known as "quantum magnetooscillations" - in the linear-response regime [see Shoenberg (1984) for a review of the basic concepts and of the results for magnetooscillations in 3D metals, and Ando et al. (1982) for an early review of magnetooscillations in a 2DEG].

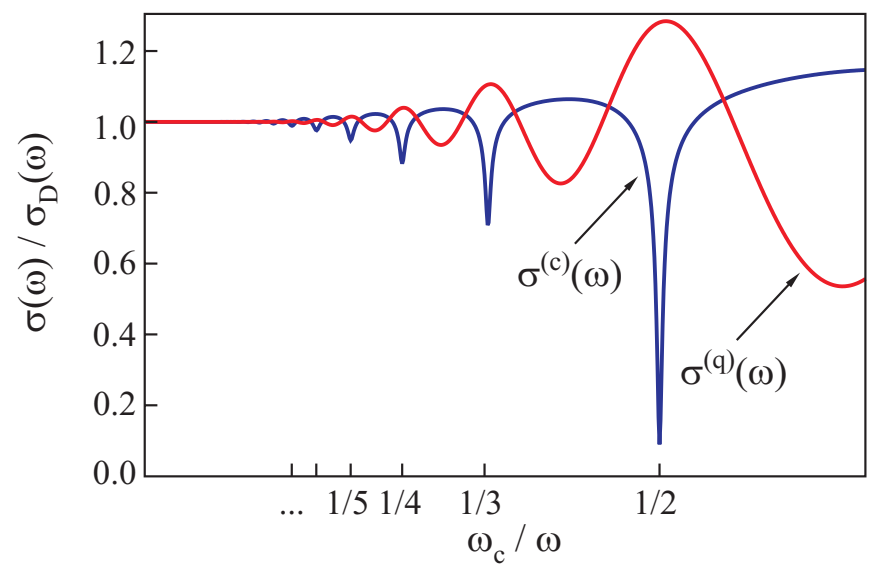

FIG. 2 Classical (c) and quantum (q) oscillatory ac conductivity [normalized to the Drude conductivity $\sigma_{\mathrm{D}}(\omega)$ ] vs $\omega_{c} / \omega$ for $\omega / 2 \pi=100 \mathrm{GHz}, \tau_{L}=0.6 \mathrm{~ns}, \tau_{L} / \tau_{\mathrm{q}}=50, \tau_{S} / \tau_{L}=0.1$, and $a / \Lambda=0.25$ at $\omega_{c} / \omega=1 / 2$. From Dmitriev et al. (2004).

\section{Magnetooscillations of the density of states}

In a clean noninteracting 2DEG the DOS per spin is a sum of delta-functions at energies equal to those of LLs:

$$
\nu(\varepsilon)=\left(2 \pi \lambda_{B}^{2}\right)^{-1} \sum_{N} \delta\left(\varepsilon-\varepsilon_{N}\right),
$$

where $\lambda_{B}=\left(m \omega_{c}\right)^{-1 / 2}$ is the magnetic length and $\varepsilon_{N}=(N+1 / 2) \omega_{c}$. As throughout the review, we concentrate on the limit of moderately strong magnetic fields in which the number of occupied LLs is large. For $N \gg 1$, the broadening of the $N$ th peak in Eq. (25) by disorder changes only weakly when $N$ changes by unity. The DOS of the $N$ th $\operatorname{LL} \nu^{(N)}(\varepsilon)$ is then written as

$$
\nu^{(N)}(\varepsilon)=-\frac{1}{2 \pi^{2} \lambda_{B}^{2}} \operatorname{Im} \frac{1}{\varepsilon-\varepsilon_{N}-\Sigma(\varepsilon)}
$$

where $\Sigma(\varepsilon)$ is the self-energy of the disorder-averaged retarded Green's function in the LL representation. The approximation is that $\Sigma(\varepsilon)$ is treated as independent of $N$. The singular character of Eq. (25) implies that $\Sigma(\varepsilon)$ cannot be calculated at the level of the Born approximation when LLs are separated, i.e., when disorder is so weak that $-\operatorname{Im} \Sigma\left(\varepsilon_{N}\right) \lesssim \omega_{c}$.

The mathematically simplest scheme to calculate $\Sigma(\varepsilon)$ is the self-consistent Born approximation (SCBA) (Ando and Uemura, 1974; Laikhtman and Altshuler, 1994; Raikh and Shahbazvan, 1993) in which $\Sigma(\varepsilon)$ obeys

$$
\Sigma(\varepsilon)=\frac{\omega_{c}}{2 \pi \tau_{\mathrm{q}}} \sum_{N} \frac{1}{\varepsilon-\varepsilon_{N}-\Sigma(\varepsilon)},
$$

where $1 / \tau_{\mathrm{q}}$ is the total (quantum) scattering rate [Eq. (6)] at $B=0$. The SCBA gives in the limit $\omega_{c} \tau_{\mathrm{q}} \gg 1$, where LLs are well separated:

$$
\nu^{(N)}(\varepsilon)=\nu_{0} \tau_{\mathrm{q}} \sqrt{\frac{2 \omega_{c}}{\pi \tau_{\mathrm{q}}}-\left(\varepsilon-\varepsilon_{N}\right)^{2}}
$$


if the argument of the square root is positive and zero otherwise, with $\nu_{0}=m / 2 \pi$ being the DOS per spin at $B=0$. Benedict and Chalker (1986) and Carra et al. (1989) showed that the SCBA - and thus the semicircle shape of the broadened LLs in Eq. (28) - are exact for white-noise disorder in the limit $N \rightarrow \infty$. For a nonzero correlation length $d$ of Gaussian (in the sense of fluctuation statistics) disorder, it was recognized by Raikh and Shahbazyan (1993) that the SCBA is a parametrically accurate approximation for $N \gg 1$ provided $d \ll \lambda_{B}$. The hard gaps in between are an artefact of the SCBA, but the exponential tails of the DOS which fill the gaps smear only slightly (for $N \gg 1$ ) the boundaries of the semicircles (Benedict, 1987; Efetov and Marikhin, 1989). The gaps predicted by the SCBA close at $\omega_{c} \tau_{\mathrm{q}}=$ $\pi / 2$ (Ando, 1974b; Laikhtman and Altshuler, 1994).

Apart from the conditions $N \gg 1$ and $d \ll \lambda_{B}$, the SCBA also assumes that disorder is weak; specifically, that at zero $B$ the conventional Born approximation is valid, which for Gaussian disorder means ${ }^{2} d \ll v_{F} \tau_{\mathrm{q}}$. Moreover, the SCBA crucially assumes that the nonGaussian component of fluctuations of the random potential can be neglected. The Gaussian character of disorder implies that individual impurities are weak and their concentration $n_{i}$ obeys $n_{i} \max \left\{d^{2}, S\right\} \gg 1$, where $S$ is the area over which disorder is averaged because of the quantum uncertainty of electron paths. For separated LLs, the characteristic $S$ is given by the area $2 \pi \lambda_{B}^{2}$ occupied by one electronic state, which is assumed within the SCBA to be much larger than $d^{2}$. The DOS for disorder with non-Gaussian statistics of fluctuations was considered beyond the SCBA by Ando $(1974 \mathrm{a}, \mathrm{b})$, by Brézin et al. (1984) for $N=0$, and by Benedict and Chalker (1986) for separated LLs in the limit $N \gg 1$. In particular, it was found in these works that within a model of deltafunction impurities there develops a singularity in the DOS at $\varepsilon=\varepsilon_{N}$ for $2 \pi n_{i} \lambda_{B}^{2}<1$, independently of the strength of the impurities.

If $\omega_{c} \tau_{\mathrm{q}} \ll 1$, Landau quantization only leads to a weak modulation of the DOS and the SCBA predicts that $\nu^{(N)}(\varepsilon)$ in this limit is described by a Lorentzian with $\operatorname{Im} \Sigma(\varepsilon)=-1 / 2 \tau_{\mathrm{q}}$. The DOS is then represented, by means of the Poisson summation formula, as a sum over harmonics in the following form: ${ }^{3}$

$$
\nu(\varepsilon)=\nu_{0}\left[1+2 \sum_{k=1}^{\infty}(-\delta)^{k} \cos \frac{2 \pi k \varepsilon}{\omega_{c}}\right]
$$

\footnotetext{
${ }^{2}$ According to Eq. (7) with $W_{q}$ from Eq. (5) and $n_{e}=n_{i}$, this condition can only be marginally satisfied for randomly distributed remote impurities.

${ }^{3}$ Equation (29) describes the asymptotic behavior of the amplitude of the $k$ th harmonic in the limit $\omega_{c} \tau_{\mathrm{q}} \ll 1$ (with exponential accuracy). In the exact SCBA formula, valid for arbitrary $\omega_{c} \tau_{\mathrm{q}}$ (Vavilov and Aleiner, 2004), each harmonic contains an extra factor $g_{k}=k^{-1} L_{k-1}^{1}\left(2 \pi k / \omega_{\mathrm{c}} \tau_{\mathrm{q}}\right)$, where $L_{k-1}^{1}$ is the generalized Laguerre polynomial.
}

where the Dingle factor (Shoenberg, 1984)

$$
\delta=\exp \left(-\pi / \omega_{c} \tau_{\mathrm{q}}\right)
$$

The first harmonic - the least damped-yields the leading oscillatory correction $\Delta \nu(\varepsilon)$ to the DOS:

$$
\Delta \nu(\varepsilon)=-2 \nu_{0} \delta \cos \left(2 \pi \varepsilon / \omega_{c}\right)
$$

For $d \gg \lambda_{B}$, the SCBA fails and instead the quasiclassical approximation in which the DOS is represented as

$$
\nu^{(N)}(\varepsilon)=\left(2 \pi \lambda_{B}^{2}\right)^{-1}\left\langle\delta\left[\varepsilon-\varepsilon_{N}-\bar{V}(\mathbf{r})\right]\right\rangle
$$

is valid (Raikh and Shahbazyan, 1993). Here

$$
\bar{V}(\mathbf{r})=\int_{0}^{2 \pi} \frac{d \theta}{2 \pi} V\left(x+R_{c} \cos \theta, y+R_{c} \sin \theta\right)
$$

is the effective random potential acting on the guiding center of the cyclotron orbit. For the case of Gaussian statistics of fluctuations of $V(\mathbf{r})$, the shape of $\nu^{(N)}(\varepsilon)$ is also Gaussian and is given (Mirlin et al., 1996; Raikh and Shahbazvan, 1993) by ${ }^{4}$

$$
\nu^{(N)}(\varepsilon)=\frac{1}{2 \pi \lambda_{B}^{2}}\left(\frac{\tau_{\mathrm{q}}}{\omega_{c}}\right)^{1 / 2} \exp \left[-\frac{\pi \tau_{\mathrm{q}}}{\omega_{c}}\left(\varepsilon-\varepsilon_{N}\right)^{2}\right]
$$

for $\lambda_{B} \ll d \ll R_{c}$ and by

$$
\nu^{(N)}(\varepsilon)=\frac{1}{2 \pi \lambda_{B}^{2}}[2 \pi W(0)]^{-1 / 2} \exp \left[-\frac{\left(\varepsilon-\varepsilon_{N}\right)^{2}}{2 W(0)}\right] \text {. }
$$

for $d \gg R_{c}$ [in which limit $\left.\bar{V}(\mathbf{r}) \simeq V(\mathbf{r})\right]$. In Eq. (35), $W(0)$ is the variance of $V(\mathbf{r})$ [Eq. (3) ]. Note that the broadening grows with increasing $B$ and saturates at $d \gg R_{c}$. Equations (34) and (35) describe in effect inhomogeneous broadening and are valid for both separated and overlapping LLs.

The sum of $\nu^{(N)}(\varepsilon)$ from Eq. (34) over $N$ gives the sum over harmonics

$$
\nu(\varepsilon)=\nu_{0}\left[1+2 \sum_{k=1}^{\infty}(-1)^{k} \delta^{k^{2}} \cos \frac{2 \pi k \varepsilon}{\omega_{c}}\right] .
$$

Note that the only difference between Eqs. (29) and (36) is in the power of the factor $\delta$ : it is $k$ in the former case and $k^{2}$ in the latter. It follows that the damping of the leading $(k=1)$ oscillatory correction in the limit of overlapping LLs is given by Eq. (31) in both cases, despite the

\footnotetext{
4 The Gaussian shape of LLs was also obtained in an early work by (Gerhardts, 1975) within the cumulant expansion of the selfenergy in the time domain to first order in $W_{q}$. However, the control parameters of the approximation were not correctly specified there. In particular, Eq. (34) was argued by (Gerhardts, 1975) to be valid for disorder with the correlation length $d \rightarrow 0$ (i.e., in the limit $d \ll \lambda$ ), whereas the actual condition is $d \gg \lambda$.
} 
shape of LLs being quite different (Lorentzian vs Gaussian). If $d \gg R_{c}$, the exponent of the damping factor for the $k$ th harmonic is proportional to $k^{2}$, similarly to Eq. (36), with the $k=1$ term in $\nu(\varepsilon)$ given by

$$
\Delta \nu(\varepsilon)=-2 \nu_{0} \exp \left[-2 \pi^{2} W(0) / \omega_{c}^{2}\right] \cos \left(2 \pi \varepsilon / \omega_{c}\right) .
$$

The Dingle plot [the term conventionally used to describe the behavior of $\ln \Delta \nu(\varepsilon)$ plotted in the low- $T$ limit as a function of $1 / B$ ] is seen to be quadratic in Eq. (37), in contrast to Eq. (31) where it is linear. The quadratic Dingle plot $^{5}$ in the DOS can thus be used to probe the presence of long-range (with the correlation radius $d \gtrsim$ $R_{c}$ ) inhomogeneities in the sample.

\section{Shubnikov-de Haas oscillations}

The magnetooscillations of the DOS give rise to the oscillatory behavior of the dc transport coefficients as $\varepsilon_{F} / \omega_{c}$ is varied - the Shubnikov-de Haas (SdH) effect. Within the SCBA (Ando and Uemura, 1974), the conductivity tensor for $N \gg 1$ was obtained for shortrange $\left(d \ll k_{F}^{-1}\right)$ disorder by Ando $(1974 \mathrm{~b})$ for the diagonal conductivity $\sigma_{x x}$ and by Ando et al. (1975) for the Hall conductivity $\sigma_{x y}$. Note that the use of the SCBA to treat also long-range disorder with $d \gg \lambda_{B}$ in the early works (Ando et al., 1982) is not justified since this condition violates the applicability of the SCBA (Raikh and Shahbazyan, 1993). A generalization of the SCBA approach which provides a framework for studying transport in the case of disorder with an arbitrary correlation length $d \ll \lambda_{B}$ was developed by Dmitriev et al. (2003). An essential ingredient of the theory describing long-range disorder $\left(d \gtrsim k_{F}^{-1}\right)$ is the inclusion of vertex corrections in averaging over disorder. The result is that the theory for all $d \ll \lambda$ can be formulated solely in terms of the oscillatory DOS $\nu(\varepsilon)$ and the transport scattering rate $\tau_{B}(\varepsilon)$ which is renormalized by Landau quantization as follows:

$$
\frac{1}{\tau_{B}(\varepsilon)}=\frac{1}{\tau} \frac{\nu(\varepsilon)}{\nu_{0}}
$$

Expressed in terms of $\nu(\varepsilon)$ and $\tau_{B}(\varepsilon), \sigma_{x x}$ (neglecting the Zeeman splitting) reads (Dmitriev et al., 2003)

$$
\sigma_{x x}=e^{2} v_{F}^{2} \int d \varepsilon\left(-\frac{\partial f_{\varepsilon}^{T}}{\partial \varepsilon}\right) \frac{\nu(\varepsilon) \tau_{B}(\varepsilon)}{1+\omega_{c}^{2} \tau_{B}^{2}(\varepsilon)},
$$

where $f_{\varepsilon}^{T}$ is the thermal distribution function. Equation (39) describes both separated and overlapping LLs and

\footnotetext{
${ }^{5}$ If the damping of the oscillations in the DOS is solely due to remote impurities, a finite range of $B$ within which LLs are not separated and the Dingle plot is quadratic exists provided disorder is sufficiently strong; specifically, if the "out-scattering length" $v_{F} \tau_{\mathrm{q}} \ll d$. In ultra-high mobility samples, $v_{F} \tau_{\mathrm{q}}$ is typically of the order of $d$.
}

is valid also in the crossover in between. Note that, compared to the Drude formula $\sigma_{\mathrm{D}}=\left(e^{2} n_{e} \tau / m\right) /\left(1+\omega_{c}^{2} \tau^{2}\right)$, Landau quantization manifests itself in Eq. (39) in two ways: in the renormalization $\tau \rightarrow \tau_{B}(\varepsilon)$ and in the appearance of the additional factor $\nu(\varepsilon) / \nu_{0}$ in the numerator [where, in view of Eq. (38), the DOS oscillations cancel out]. As $B$ is varied, $\sigma_{x x}$ at zero $T$ is represented in the limit of separated LLs as a series of peaks the height of which is given by $\sigma_{x x}^{\max } \simeq \sigma_{\mathrm{D}}\left(2 \omega_{c} \tau_{\mathrm{q}} / \pi\right) \gg \sigma_{\mathrm{D}}$. For overlapping LLs, the oscillatory correction $\Delta \sigma$ to $\sigma_{\mathrm{D}}$ reads

$$
\frac{\Delta \sigma}{\sigma_{\mathrm{D}}} \simeq \frac{2 \omega_{c}^{2} \tau^{2}}{1+\omega_{c}^{2} \tau^{2}} \frac{\Delta \nu\left(\varepsilon_{F}\right)}{\nu_{0}} \mathcal{F}\left(\frac{2 \pi^{2} T}{\omega_{c}}\right),
$$

where $\Delta \nu(\varepsilon)$ is given by Eq. (31) and the factor $\mathcal{F}(x)=$ $x / \sinh x$ describes the thermal averaging of the oscillations. Importantly, in the case of long-range disorder $\left(k_{F} d \gg 1\right)$, the magnetic field may be "classically strong" in the sense of $\omega_{c} \tau \gg 1$ and at the same time, if $\omega_{c} \tau_{\mathrm{q}} \ll 1$, lead to only weak $\mathrm{SdH}$ oscillations even at $T=0$. Note also that for $T \gg \omega_{c} \gg \tau_{\mathrm{q}}^{-1}, \tau^{-1}$ the thermal averaging over the contributions of separated LLs in Eq. (39) leads to a nonoscillatory contribution to the MR

$$
\rho_{x x} / \rho_{\mathrm{D}}=(8 / 3 \pi)\left(2 \omega_{c} \tau_{\mathrm{q}} / \pi\right)^{1 / 2} .
$$

Extending Eq. (38) to the Hall conductivity yields

$$
\sigma_{x y}=-\frac{e n_{e} c}{B}+\frac{e^{2} v_{F}^{2}}{\omega_{c}} \int d \varepsilon\left(-\frac{\partial f_{\varepsilon}^{T}}{\partial \varepsilon}\right) \frac{\nu(\varepsilon)}{1+\omega_{c}^{2} \tau_{B}^{2}(\varepsilon)},
$$

where the first term describes a collisionless drift of electrons in crossed electric and magnetic fields. The oscillatory MR for overlapping LLs is then given by ${ }^{6}$

$$
\frac{\Delta \rho}{\rho_{\mathrm{D}}} \simeq 2 \frac{\Delta \nu\left(\varepsilon_{F}\right)}{\nu_{0}} \mathcal{F}\left(\frac{2 \pi^{2} T}{\omega_{c}}\right)
$$

for arbitrary $\omega_{c} \tau \ll \tau / \tau_{\mathrm{q}}$. It is worth noting that the main contribution to the oscillatory MR (43) comes from the oscillations of $\sigma_{x x}$ for $\omega_{c} \tau \gg 1$ and from the oscillations of $\sigma_{x y}$ for $\omega_{c} \tau \ll 1$. In the limit of white-noise disorder $\left(\tau=\tau_{\mathrm{g}}\right)$, Eqs. (39) and (42) agree with those derived by Ando (1974b, Eq. (2.12)) and by Ando et al. (1975), Eq. (3.26)), respectively. ${ }^{7}$ In the case of longrange disorder, they confirm the form of $\mathrm{SdH}$ oscillations hypothesized by Coleridge et al. (1989).

It is worth noting that the Fermi energy $\varepsilon_{F}$ for a fixed electron density $n_{e}$ also exhibits magnetooscillations. These are qualitatively important for the dependence of the MR on $B$ in the case of separated LLs at

\footnotetext{
6 Inelastic electron-electron scattering (or, for that matter, any scattering whose strength is proportional to $\varepsilon^{2}+\pi^{2} T^{2}$ ) does not lead to an additional exponential damping of the $\mathrm{SdH}$ oscillations for $T \gg \omega_{c}$ (Adamov et al., 2006; Martin et al., 2003).

7 The expansion of $\sigma_{x y}$ to first order in $\delta$ obtained by Ando et al. (1975) was also derived by means of a different representation of the Kubo formula for Hall transport by Isihara and Smrčka (1986).
} 
zero $T$ : it is because of the oscillations of $\varepsilon_{F}$ that $\rho_{x x}$ as a function of $B$ does not have gaps similar to those in the dependence of $\nu(\varepsilon)$. For overlapping LLs, the oscillations of $\varepsilon_{F}$ yield only a subleading (in $\omega_{c} / \varepsilon_{F} \ll 1$ ) oscillatory term in the MR. The thermal averaging for $T \gg \omega_{c}$ suppresses the oscillations of $\varepsilon_{F}$ exponentially, similarly to Eq. (40).

As discussed in Sec. II.A, disorder in high-mobility heterostructures is a mix of long- and short-range components characterized by vastly different correlation radii. Because of the large ratio $\tau / \tau_{\mathrm{q}}$ for scattering off the longrange component of disorder, it is possible that the mobility $\mu$ and the Dingle factor $\delta$ are determined by different sources of disorder. Specifically, it is likely that, in ultra-high mobility samples, $\mu$ is limited by background impurities and interface roughness, whereas the damping of the magnetooscillations is mainly due to scattering off remote impurities.

Note also that, in real samples, there may exist ultralong range inhomogeneities with a correlation radius larger than $R_{c}$ in the regime of $\mathrm{SdH}$ oscillations. These inhomogeneities may not affect the mobility but will provide an additional damping of the oscillations of $\Delta \rho / \rho$ in Eq. (43), which is described by the exponential factor in Eq. (37). If $W(0) \gg \omega_{c} / \tau_{q}$, the damping of the $\mathrm{SdH}$ oscillations will be mainly determined by these macroscopic inhomogeneities and the Dingle plot for the MR will be quadratic in $1 / B$, as was indeed reported for some $\mathrm{SdH}$ measurements (e.g., Coleridge, 1991, 1997).

So far, the magnetooscillations of the MR and the DOS have been directly related to each other, being characterized by the same Dingle factors. One important reason why this is not true in general is the suppression of the transport coefficients by localization effects. These come in two varieties: classical and quantum. An example of classical localization is the adiabatic drift along equipotential lines of a smooth random potential, which results in the exponential suppression (Fogler et al., 1997) of $\sigma_{x x}$ with increasing $B$ [Eq. (17)]. Oscillations of the MR are then associated with the oscillatory DOS for only a subset of electron paths; specifically, for those giving the main contribution to $\sigma_{x x}$. The statistical properties of disorder present along the "conducting" paths do not necessarily coincide with those on average, which leads in general to different Dingle factors for the DOS and the MR. The difference was shown (Evers et al., 1999) to be very pronounced for the case of a spatially random magnetic field.

Quantum localization becomes strong when $\sigma_{x x}$ drops with increasing $B$ to a value of the order of the conductance quantum $e^{2} / 2 \pi$, at which field the $\mathrm{QH}$ effect sets in. ${ }^{8}$ Importantly, if remote impurities, distributed ran-

\footnotetext{
8 Strictly speaking, single-particle states of a $2 \mathrm{DEG}$ at $B \neq 0$ are localized except those at a discrete set of critical energies - this localization constitutes the essence of the QH effect. However, the temperature needed to observe localization is exponentially small if $\sigma_{x x} \gg e^{2} / 2 \pi$ in the absence of localization, so that in
}

domly with the density $n_{i}=n_{e}$, are the only source of disorder, the cyclotron frequency for the crossover field $B_{\text {loc }}$ obeys $\omega_{c} \tau_{\mathrm{q}} \sim\left(k_{F} d\right)^{-1 / 2} \ll 1$, i.e., strong localization develops when LLs are still well overlapped and the DOS does not exhibit any gaps. If, however, the shortrange component of disorder substantially decreases $\tau$ while not affecting $\tau_{\mathrm{q}}$ (which is the likely situation in ultra-high mobility structures, see above), $B_{\text {loc }}$ shifts upward. It follows that it is only because of the admixture of sufficiently strong short-range disorder that developed SdH oscillations can be observed in high mobility samples before the crossover to the $\mathrm{QH}$ regime.

At this point, it is also worth emphasizing that quantum magnetooscillations of the MR may not be related to those of the DOS at all. Even if the latter are neglected, the quantum interference of diffusive waves for $\sigma_{x x} \gg e^{2} / 2 \pi$ gives rise to the $\mathrm{QH}$ oscillations $\Delta \sigma_{x x} \propto$ $\cos \left(2 \pi g_{x y}\right) \exp \left(-2 \pi g_{x x}\right)$, where $g_{x x, x y}$ is $\sigma_{x x, x y}$ in units of $e^{2} / 2 \pi$ (Pruisken, 1990). These are the oscillations into which the QH effect transforms as $B$ is decreased-not into the $\mathrm{SdH}$ oscillations, contrary to the common belief. Note that the period of the QH oscillations corresponds to one flux quantum through the area $\min \left\{l^{2}, R_{c}^{2}\right\}$. That is, the $B$-dependent interference occurs within the area the size of which is not related to (and is smaller than) the localization length, so that the observation of these oscillations for $\sigma_{x x} \gg e^{2} / 2 \pi$ does not require exponentially low $T$-in contrast to the QH plateaus. See Mirlin et al. (1998) and Evers et al. (1999) for a discussion of the interplay of the $\mathrm{SdH}$ and $\mathrm{QH}$ oscillations in the case of a random magnetic field.

\section{Quantum magnetooscillations of the ac conductivity}

The oscillations of the DOS induced by Landau quantization have important consequences also for ac transport. Specifically, the Drude formula in Eq. (22) for $\sigma_{ \pm}(\omega)$, which describes a single peak of the CR, and Eq. (39) for the oscillatory $\sigma_{x x}$ at $\omega=0$ generalize $^{9}$ to (Dmitriev et al., 2003)

$$
\begin{aligned}
& \sigma_{ \pm}(\omega)=\frac{e^{2} v_{F}^{2}}{2 \omega} \\
& \times \int d \varepsilon \frac{\left(f_{\varepsilon}^{T}-f_{\varepsilon+\omega}^{T}\right) \nu(\varepsilon) \tau_{B}^{-1}(\varepsilon+\omega)}{\left[\tau_{B}^{-2}(\varepsilon)+\tau_{B}^{-2}(\varepsilon+\omega)\right] / 2+\left(\omega \pm \omega_{c}\right)^{2}}
\end{aligned}
$$

reality the $\mathrm{QH}$ effect becomes pronounced only when $\sigma_{x x}$ per spin drops down to the values of a few conductance quanta.

9 Note that Eq. (44) differs in an essential way from the expression for $\sigma_{ \pm}(\omega)$ obtained by Ando (1975) (for the case of shortrange disorder). In particular, the $\varepsilon$-dependent factors in the integrand of Eq. (44) do not reduce near the CR to the simple product $\left(f_{\varepsilon}^{T}-f_{\varepsilon+\omega}^{T}\right) \nu(\varepsilon) \nu(\varepsilon+\omega)$ in the limit of separated LLs, as suggested by Ando (1975) for the CR lineshape. 
At zero $T$, for the Fermi energy lying between two separated LLs, Eq. (44) gives a CR peak whose height ${ }^{10}$

$$
\sigma_{-}\left(\omega_{c}\right)=\left(e^{2} n_{e} \tau / m\right)\left(2 / \pi \omega_{c} \tau_{\mathrm{q}}\right)^{1 / 2}
$$

is much smaller than that following from the Drude theory [Eq. (22)]. In the case of long-range disorder $\left(\tau \gg \tau_{\mathrm{q}}\right)$, the CR lineshape from Eq. (44) is given by

$$
\frac{\sigma_{-}(\omega)}{\sigma_{-}\left(\omega_{c}\right)}=f\left(\frac{\omega-\omega_{c}}{\Delta_{\mathrm{CR}}}\right)
$$

where

$$
f(x)=1-\frac{x^{2}}{2 \sqrt{1+x^{2}}} \ln \frac{\sqrt{1+x^{2}}+1}{\sqrt{1+x^{2}}-1}
$$

and

$$
\Delta_{\mathrm{CR}}=\left(2 \omega_{c} \tau_{\mathrm{q}} / \pi\right)^{1 / 2} / \tau .
$$

The width of the CR for separated LLs $\Delta_{\mathrm{CR}}$ is seen to be much larger than $1 / \tau$. At the same time, in the case of long-range disorder, the CR as a function of $\omega$ is much narrower than the peaks in the DOS.

Landau quantization in combination with disorder leads to the emergence of $\mathrm{CR}$ harmonics at $\omega=M \omega_{c}$ with $|M|=2,3, \ldots$ (Ando, 1975). For separated LLs, Eq. (44) gives for the height of the $M$ th peak

$$
\sigma\left(M \omega_{c}\right)=\frac{4}{3 \pi} \frac{e^{2} n_{e}}{m \omega_{c}}\left(\frac{2}{\pi \omega_{c} \tau_{\mathrm{q}}}\right)^{1 / 2} \frac{\tau_{\mathrm{q}}}{\tau} \frac{M^{2}+1}{\left(M^{2}-1\right)^{2}},
$$

which is seen to be smaller by the factor of $\tau_{\mathrm{q}} / \tau$ than the result (Ando, 1975) for the case of short-range disorder. In contrast to the CR peak, the width of the higherharmonic peaks is given by the width of the peaks in the DOS. Thus, if one imagines that the correlation radius of disorder is increased at fixed $\tau$, the CR peak becomes higher and narrower, while the tendency for the higherharmonic peaks is the opposite.

For strongly overlapping LLs, the dynamic response of the 2DEG is given by the CR, which is described for $\omega_{c} \tau_{\mathrm{q}} \ll 1$ by the Drude formula (22), and by the $\omega$ dependent analog of the $\mathrm{SdH}$ oscillations (40). To first order in $\delta$, the oscillatory correction $\Delta \sigma_{+}(\omega)$ to the Drude conductivity reads (Dmitriev et al., 2003)

$$
\frac{\Delta \sigma_{ \pm}(\omega)}{\sigma_{\mathrm{D}, \pm}(\omega)} \simeq \frac{\Delta \nu\left(\varepsilon_{F}\right)}{\nu_{0}} \mathcal{F}\left(\frac{2 \pi^{2} T}{\omega_{c}}\right) \chi_{1}
$$

where

$$
\chi_{1}=\frac{\Omega_{ \pm}^{2}}{1+\Omega_{ \pm}^{2}} \frac{\omega_{c}}{\pi \omega}\left(\sin \frac{2 \pi \omega}{\omega_{c}}+\frac{1+3 \Omega_{ \pm}^{2}}{\Omega_{ \pm}^{3}} \sin ^{2} \frac{\pi \omega}{\omega_{c}}\right)
$$

10 The peak height found by Ando (1975) for short-range disorder is larger than that given by Eq. (45) at $\tau=\tau_{\mathrm{q}}$ by a factor of $4 / 3$.

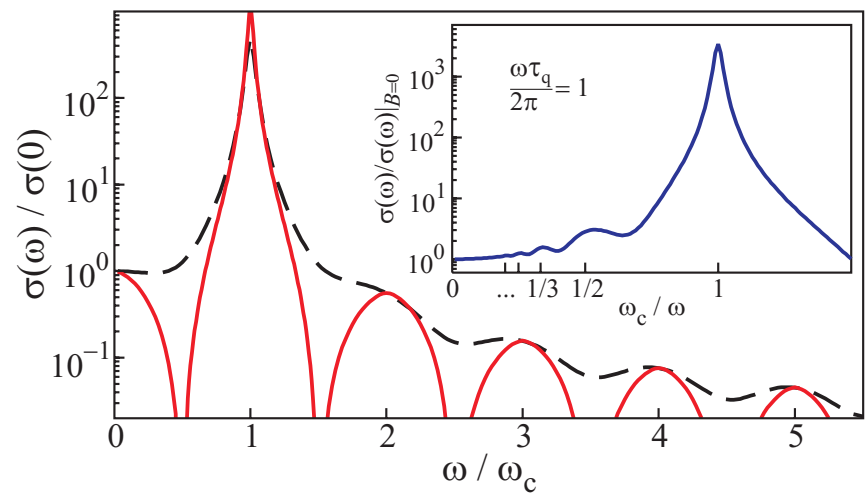

FIG. 3 Magnetooscillations of the ac conductivity of a 2DEG with smooth disorder $\left(\tau / \tau_{\mathrm{q}}=10\right)$ in the limit $T \gg T_{D}$, in units of the dc conductivity, as a function of $\omega / \omega_{c}$ for fixed $\omega_{c} \tau_{\mathrm{q}} / \pi=3.25$ (solid line) and 1 (dashed). Inset: the conductivity vs $\omega_{c} / \omega$ for fixed $\omega \tau_{\mathrm{q}} / 2 \pi=1$ in units of the ac conductivity at $B=0$. From Dmitriev et al. (2003).

and $\Omega_{ \pm}=\left(\omega \pm \omega_{c}\right) \tau$. In the dc limit, Eq. (50) reduces to Eq. (40). If $T$ is much larger than the Dingle temperature $T_{D}=1 / 2 \pi \tau_{\mathrm{q}}$, the main source of damping of the oscillations with varying $\varepsilon_{F} / \omega_{c}$ is the thermal averaging. In ultra-high mobility heterostructures, $T_{D} \sim 10^{2} \mathrm{mK}$ and for the typical measurement temperature $T \sim 1 \mathrm{~K}$ the linear-in- $\delta$ oscillations (50) are completely washed out.

Unlike the oscillations with $\varepsilon_{F} / \omega_{c}$, which constitute the essence of the $\mathrm{SdH}$ effect in the dc case, the oscillations with $\omega / \omega_{c}$ survive the thermal averaging. Specifically, they survive in the even-order terms in the expansion of $\sigma_{ \pm}(\omega)$ in powers of $\delta$, in which the intermodulation of the DOS oscillations at energies separated by $\omega$ does not go away upon averaging over energy. For strongly overlapping LLs, the oscillatory behavior of $\sigma_{ \pm}(\omega)$ as $\omega / \omega_{c}$ is varied at $T \gg T_{D}$ comes from the correction $\Delta \sigma_{+}(\omega)$ of order $\delta^{2}$, which in the high- $T$ limit is given by (Dmitriev et al., 2003)

$$
\Delta \sigma_{ \pm}(\omega) / \sigma_{\mathrm{D}, \pm}(\omega) \simeq 2 \delta^{2} \chi_{2}
$$

with

$$
\begin{aligned}
\chi_{2} & =\frac{\Omega_{ \pm}^{2}}{\left(1+\Omega_{ \pm}^{2}\right)^{2}} \\
& \times\left[\left(\Omega_{ \pm}^{2}-3\right) \cos \frac{2 \pi \omega}{\omega_{c}}+\frac{3 \Omega_{ \pm}^{2}-1}{\Omega_{ \pm}} \sin \frac{2 \pi \omega}{\omega_{c}}\right] .
\end{aligned}
$$

As noted already in the discussion of the $\mathrm{SdH}$ effect, in the case of long-range disorder, for a classically strong magnetic field with $\omega_{c} \tau \gg 1$, the modulation of the DOS is still small as long as $\omega_{c} \tau_{\mathrm{q}} \ll 1$. Then, away from the CR (for $\left|\Omega_{ \pm}\right| \gg 1$ ) Eq. (52) reduces to the simple form

$$
\Delta \sigma_{ \pm}(\omega) / \sigma_{\mathrm{D}, \pm}(\omega) \simeq 2 \delta^{2} \cos \left(2 \pi \omega / \omega_{c}\right) .
$$

The overall behavior of the $T$-independent oscillations of $\sigma(\omega)$ is illustrated in Fig. 3 . 
Intimately related to the independence of Eqs. (52)(54) on $T$ is the fact that the oscillations with $\omega / \omega_{c}$ are not sensitive to the presence of macroscopic inhomogeneities (which may complicate the determination of $\tau_{\mathrm{q}}$ from $\mathrm{SdH}$ experiments, as discussed at the end of Sec. II.C.2). The measurement of the damping of the $\omega / \omega_{c}-$ oscillations as a function of $B$ in the high- $T$ regime may thus be the most reliable means of extracting $\tau_{\mathrm{q}}$ from the magneto-oscillatory behavior of the transport coefficients. Note, however, that the CR harmonics-and the related periodic modulation of $\sigma_{ \pm}(\omega)$ with $\omega / \omega_{c}-$ may be associated not only with Landau quantization but also with the quasiclassical memory effects discussed in Sec. II.B.3. In fact, the latter can produce strong oscillations of $\sigma_{ \pm}(\omega)$ with an amplitude comparable to the Drude conductivity $\sigma_{\mathrm{D}, \pm}(\omega)$ [see Fig. 2, for more details of the comparison between these two sources of the magnetooscillations in the ac conductivity and the dc photoconductivity see Dmitriev et al. (2004)].

The oscillations of $\sigma_{ \pm}(\omega)$ as $\omega / \omega_{c}$ is varied on the low- $B$ side of the $C R$, as well as the oscillations of the dynamic conductivity with $\varepsilon_{F} / \omega_{c}$, have been observed in the early studies by Abstreiter et al. (1976) in the far-infrared light transmission experiments on a low-mobility 2DEG in Si inversion layers. However, only recently both these types of magnetooscillations have also been observed in microwave absorption experiments on high-mobility GaAs/AlGaAs heterostructures (Fedorych et al., 2010). One reason why the measurements of $\sigma_{ \pm}(\omega)$ in the absorption/transmission experiments may be substantially complicated is that the conductivity expresses the current as a response to the total (screened) electric field whereas what is probed in this type of experiments is a response to the (unscreened) field of the incident electromagnetic wave. As a result, the behavior of $\sigma_{ \pm}(\omega)$ in the absorption/transmission may be masked by the excitation of magnetoplasmons (Fedorych et al., 2010; Studenikin et al., 2007; Wirthmann et al., 2007).

One more point - specific to a high-mobility 2DEG with $\left|\sigma_{x x}(\omega) \pm i \sigma_{x y}(\omega)\right| \gg c n_{r}$, where $c$ is the speed of light in the vacuum and $n_{r}$ is the refractive index of the medium on the sides of the $2 \mathrm{DEG}$ - is a strong reflection of the electromagnetic wave caused by the dynamical screening inside the 2DEG (Chiu et al., 1976; Fal'ko and Khmel'nitskii, 1989). In a magnetic field, the enhanced reflection leads to a suppression and an additional broadening of the CR (Chiu et al., 1976; Mikhailov, 2004) in the absorption/transmission coefficient. For a linearly polarized wave normally incident on the $2 \mathrm{DEG}$, the absorption coefficient $\mathrm{A}=\mathrm{A}_{+}+\mathrm{A}_{-}$and

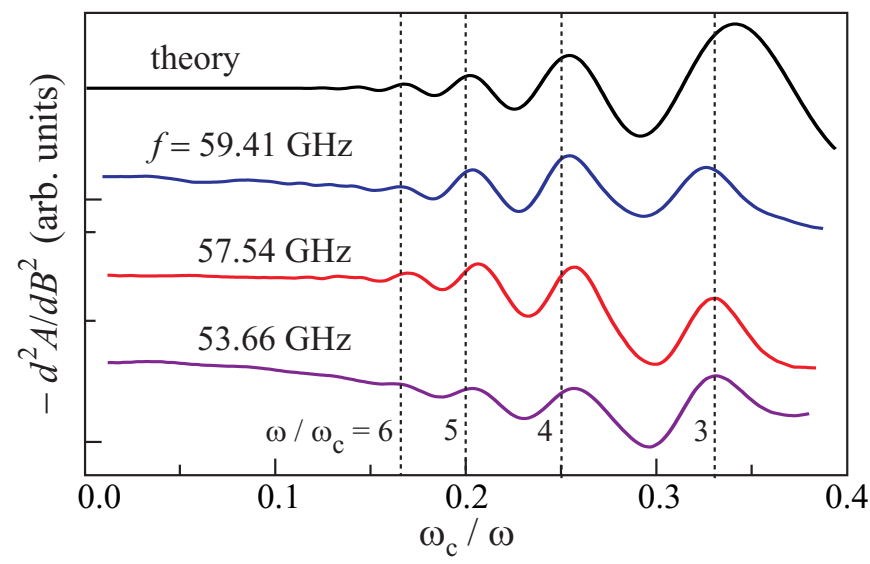

FIG. 4 Absorption coefficient A, differentiated twice with respect to $B$, as a function of $\omega_{c} / \omega$ for fixed $\omega$. Three lower curves, marked by different radiation frequencies $\omega / 2 \pi$, were taken at $T=2 \mathrm{~K}$ in a sample with $n_{e} \simeq 3.6 \times 10^{11} \mathrm{~cm}^{-2}$ and $\mu \simeq 5 \times 10^{6} \mathrm{~cm}^{2} / \mathrm{Vs}$. The upper curve is the calculated dependence of $-d^{2} \mathrm{~A} / d B^{2}$ on $\omega_{c} / \omega$ at fixed $\omega$ according to Eqs. (54) and (55) with $\tau_{\mathrm{q}}=9.1 \mathrm{ps}$ determined from the oscillatory-photoconductivity experiment. The curves are offset for clarity. From Fedorvch et al. (2010).

the transmission coefficient $\mathrm{T}=\mathrm{T}_{+}+\mathrm{T}_{-}$are given by ${ }^{11}$

$$
\mathrm{A}_{ \pm}=\operatorname{Re} g_{ \pm} /\left|1+g_{ \pm}\right|^{2}, \quad \mathrm{~T}_{ \pm}=1 / 2\left|1+g_{ \pm}\right|^{2},
$$

where $g_{ \pm}=2 \pi\left(\sigma_{x x} \pm i \sigma_{x y}\right) / c n_{r}$ (neglecting the difference in the dielectric properties of GaAs and AlGaAs). ${ }^{12}$ In the limit where the Drude formula for $g_{ \pm}$can be applied,

$$
\mathrm{A}_{\mathrm{D}, \pm}=\frac{2 \gamma \tau}{1+\Omega_{ \pm}^{2}} \mathrm{~T}_{\mathrm{D}, \pm}=\frac{\gamma \tau}{(1+\gamma \tau)^{2}+\Omega_{ \pm}^{2}}
$$

and the dynamical-screening-induced broadening

$$
\gamma=2 \pi e^{2} n_{e} / m c n_{r}
$$

In ultra-high mobility GaAs/AlGaAs heterostructures, the product $\gamma \tau$ may be as large as a few tens. A sub-

11 Note that Eqs. (55) describe scattering by the 2DEG itself. To relate them to the absorption and transmission coefficients of the electromagnetic wave incident on the sample, one should take into account multiple Fresnel reflections from the boundaries of the latter. Changes of the refractive index on scales smaller than the wavelength of the radiation are averaged. If the wavelength inside the sample $2 \pi c / \omega n_{r}$ is much larger than the thickness of the sample, one can neglect the polarization of the medium around the 2DEG and put $n_{r} \rightarrow 1$ in $g_{ \pm}$and $\gamma$. When the distance from the 2DEG to the top surface of the heterostructure is much smaller than the wavelength (which is a typical situation), scattering by the 2DEG and the top surface (Fedorych et al., 2010) is described by Eqs. (55) with the change $n_{r} \rightarrow\left(1+n_{r}\right) / 2$ in $g_{ \pm}$with a simultaneous multiplication of $\mathrm{A}_{ \pm}$ and $\mathrm{T}_{ \pm}$by $2 n_{r} /\left(1+n_{r}\right)$ and $4 n_{r} /\left(1+n_{r}\right)^{2}$, respectively.

12 To avoid notational confusion: the abbreviation $\sigma_{ \pm}$is used in this review to denote $\operatorname{Re}\left(\sigma_{x x} \pm i \sigma_{y x}\right) / 2$ (as, e.g., in Dmitriev et al., 2003), whereas in a number of relevant papers (e.g., Fedorvch et al., 2010) $\sigma_{ \pm}$denotes $\sigma_{x x} \pm i \sigma_{y x}$. 
stantially enhanced - compared to the transport scattering rate - broadening of the $\mathrm{CR}$ in the absorption (for magnetic fields in which the LLs were not much separated) was reported in a high-mobility structure by Studenikin et al. (2005), similar to the very strong broadening of the CR in the transmission through ultra-high mobility structures (Smet et al., 2005; ;ung et al., 2009). The oscillatory behavior of the absorption coefficient as a function of $\omega / \omega_{c}$, observed by Fedorvch et al. (2010), was related therein to the oscillations of the conductivity tensor through Eq. (55). The low- $B$ portion of the data shows good agreement with the simple asymptotic

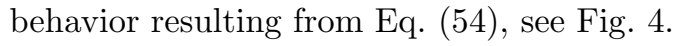

\section{Intersubband magnetooscillations}

The mechanism in Sec. II.C.3 that relates the $\omega / \omega_{c^{-}}$ oscillations of $\sigma(\omega)$-which survive the thermal averaging [Eq. (52)] - to the intermodulation of the DOS oscillations at energies separated by $\omega$ can also lead to $T$-independent magnetooscillations in dc transport. For that, one needs two (or more) parallel 2D electron gases, allowing for the exchange of electrons between them, with the bottoms of their energy bands located at different energies. One system of this type is a QW with two lowest subbands occupied by electrons, for which Polvanovsky (1988) and Leadley et al. (1992) pointed out that there should exist oscillations of the MR periodic in $\Delta / \omega_{c}$, where $\Delta$ is the subband spacing, not suppressed by the thermal averaging. The SCBA approach to describe $\mathrm{SdH}$ oscillations in $\sigma_{x x}$ was generalized for short-range disorder to the case of two subbands by Raikh and Shahbazvan (1994) (see, however, footnote 14 below). The SCBA theory of SdH oscillations in the two-subband conductivity tensor for an arbitrary type of disorder was developed by Raichev (2008).

The correction $\Delta \sigma_{\text {miso }}$ to the dissipative Drude conductivity, which describes the magnetointersubband oscillations (MISO), is a sum of two terms $\Delta \sigma_{\text {miso, } \alpha}$ with $\alpha=1,2$, coming from electrons in the $\alpha$ th subband. For $\omega_{c} \tau_{\alpha} \ll 1$, the partial contributions $\Delta \sigma_{\text {miso, } \alpha}$ are given for white-noise disorder by (Averkiev et al., 2001; Raichev, 2008)

$$
\Delta \sigma_{\mathrm{miso}, \alpha}=\frac{2 e^{2} n_{\alpha} \tau_{\alpha}^{2}}{m \tau_{12}}\left(1-\frac{2 \tau_{\alpha}}{\tau_{12}}\right) \delta_{1} \delta_{2} \cos \frac{2 \pi \Delta}{\omega_{c}},
$$

where $n_{\alpha}$ and $1 / \tau_{\alpha}$ are the electron density and the zero$B$ scattering rate in the $\alpha$ th subband, $1 / \tau_{\alpha}$ includes both intra- and intersubband scattering, $1 / \tau_{12}$ is the zero- $B$ intersubband scattering rate, and $\delta_{\alpha}$ is given by Eq. (30) with $\tau_{\alpha}$ substituted for $\tau_{\mathrm{q}}$. Equation (58) follows ${ }^{13}$ directly from Eq. (39). Importantly, Eq. (38) does not

13 The MISO amplitude resulting from Eq. 39) and the one obtained by Raichev (2008) agree with each other for arbitrary hold for the relation between the oscillatory DOS $\nu_{\alpha}(\varepsilon)$ and the oscillatory scattering rate $\tau_{B, \alpha}(\varepsilon)$ in the $\alpha$ th subband, since $1 / \tau_{B, \alpha}(\varepsilon)$ contains the rate of intersubband scattering, whereas $\nu_{\alpha}(\varepsilon)$ does not (in the leading approximation in $1 / \varepsilon_{F, \alpha} \tau_{12}$, where $\varepsilon_{F, \alpha}$ is the Fermi energy in the $\alpha$ th subband counted from its bottom). ${ }^{14}$ This is the reason why the preexponential factor in Eq. (58) does not vanish at $B \rightarrow 0$, in contrast to Eq. (52) at $\omega=0$. As a result, the MISO contribution to the MR $\Delta \rho_{\text {miso }} / \rho_{\mathrm{D}} \simeq-\Delta \sigma_{\text {miso }} \rho_{\mathrm{D}}$ at $\omega_{c} \tau_{\alpha} \ll 1$ is mainly given by the oscillatory dissipative component of the conductivity tensor (in contrast to the oscillatory dc MR in the single-subband case, see Sec. II.C.2).

Within the SCBA, Eqs. (39) and (42) allow one to describe MISO for long-range disorder as well. Similar to Sec. II.C.2 the scattering rate in the exponent of $\delta_{\alpha}$ remains then the quantum scattering rate, while $1 / \tau_{B, \alpha}(\varepsilon)$ becomes the momentum relaxation rate. However, in general, if scattering is not isotropic, the zero- $B$ momentum relaxation rate in the $\alpha$ th subband $1 / \tau_{\alpha}$ is related to the intra- and intersubband scattering rates in a nonlinear manner (Ando et al., 1982; Mamani et al., 2009b; Raichev, 2008; Zaremba, 1992). It is only in one simple case, when the intersubband scattering is isotropic, that $1 / \tau_{\alpha}$ is still given by a sum of the momentum relaxation rate for (not necessarily isotropic) scattering within the subband and the intersubband scattering rate $1 / \tau_{12}$. In this case, ${ }^{15}$ Eq. (58) for $\omega_{c} \tau_{\alpha} \ll 1$ is reproduced also for long-range disorder.

The cumbersome expressions for the MISO amplitudes in $\sigma_{x x, x y}$ for the general case of anisotropic scattering (Raichev, 2008) simplify significantly in the limit of a classically strong magnetic field $\left(\omega_{c} \tau_{\alpha} \gg 1\right)$ but overlapping LLs $\left(\delta_{\alpha} \ll 1\right)$-which conditions are only compatible in the case of long-range disorder. In this limit (Mamani et al., 2009b),

$$
\Delta \sigma_{\text {miso }}=\frac{2 e^{2}\left(n_{1}+n_{2}\right)}{m \omega_{c}^{2} \bar{\tau}_{12}} \delta_{1} \delta_{2} \cos \frac{2 \pi \Delta}{\omega_{c}}
$$

$\omega_{c} \tau_{\alpha}$, whereas the one obtained by Averkiev et al. (2001) coincides with them only for $\omega_{c} \tau_{\alpha} \rightarrow 0$.

14 At order $\mathcal{O}\left(\delta_{1} \delta_{2}\right)$, there are two contributions to MISO for $\omega_{c} \tau_{\alpha} \ll 1$ : one comes from the thermal averaging of the cross term between the oscillations in $\nu_{\alpha}(\varepsilon)$ and those in $\tau_{B, \alpha}(\varepsilon)$, the other-from the averaging of $\tau_{B, \alpha}(\varepsilon)$ alone. In Eq. (58), the former brings -1 to the expression in the brackets, the latter brings $2\left(1-\tau_{\alpha} / \tau_{12}\right)$. In the early work by Raikh and Shahbazvan (1994) [see also an extension of the theory to essentially similar oscillations in biased bilayer graphene (Mkhitaryan and Raikh, 2011)], only the former contribution was taken into account.

15 The isotropy condition for intersubband transitions requires that the Fermi wavelength in each of the subbands be much larger than $\min \{w, d\}$. As discussed in Sec. II.A this condition is only marginally satisfied in high-mobility GaAs/AlGaAs structures. 
and, correspondingly, the MISO term ${ }^{16}$ in the MR

$$
\frac{\Delta \rho_{\text {miso }}}{\rho_{\mathrm{D}}}=\frac{2}{\bar{\tau}_{12}} \frac{n_{1} \tau_{1}+n_{2} \tau_{2}}{n_{1}+n_{2}} \delta_{1} \delta_{2} \cos \frac{2 \pi \Delta}{\omega_{c}},
$$

where $1 / \bar{\tau}_{12}$ is given by an integral over the scattering angle $\phi$ [cf. Eq. (2)]:

$$
\frac{1}{\bar{\tau}_{12}}=m \int_{0}^{2 \pi} \frac{d \phi}{2 \pi}\left(1-\frac{2 \sqrt{n_{1} n_{2}}}{n_{1}+n_{2}} \cos \phi\right) W_{q}^{(12)},
$$

with $W_{q}^{(12)}$ being the Fourier component of the correlation function of the intersubband matrix element of the random potential at the transferred in-plane momentum $q=\left[2 m\left(\varepsilon_{F, 1}+\varepsilon_{F, 2}-2 \sqrt{\varepsilon_{F, 1} \varepsilon_{F, 2}} \cos \phi\right)\right]^{1 / 2}$.

Oscillations similar to MISO occur also in a double QW with the lowest subband states split by tunneling or, for that matter, in any other system whose energy spectrum exhibits two or more series of LLs, which are offset with respect to each other, in the presence of scattering between the series. Experimentally, MISO were studied in two-subband single QWs (Bvkov et al., 2010a; Coleridge, 1990; Goran et al., 2009; Leadlev et al., 1992, 1989; Rowe et al., 2001; Sander et al., 1998), in double (Bvkov, 2008b; Mamani et al., 2008) and triple (Wiedmann et al., 2009b) QWs, and in wide QWs split in two layers electrostatically Wiedmann et al., 2010a). MISO were also studied theoretically in 2D layers with electron states split by spin-orbit interaction (Langenbuch et al., 2004). The behavior of MISO in nonequilibrium conditions is discussed in Secs. III.C.4 and V.C

\section{MICROWAVE-INDUCED RESISTANCE OSCILLATIONS (MIRO)}

\section{A. MIRO: Experimental discovery and basic properties}

When a sufficiently high-mobility 2DEG is subject to a weak magnetic field and illuminated by microwave radiation, the longitudinal magnetoresistivity $\rho(B)$ exhibits giant oscillations (Zudov et al., 1997, 2001a), termed the microwave-induced resistance oscillations (MIRO), see Fig. [5. In a Corbino disk-shaped 2DEG, experiments revealed corresponding microwave-induced conductance oscillations (Yang et al., 2003). Most commonly, MIRO are observed in dc measurements, with radiation being delivered to the 2DEG via oversize waveguides [although other means, such as planar microwave transmission lines patterned on top of the 2DEG (Ye et al., 2001) and dipole antennas (Willett et al., 2004) have also been successfully implemented]. Recently, they were also observed by

16 The expression for $\Delta \rho_{\text {miso }}$ in terms of the thermally averaged product $\nu(\varepsilon) \nu(\varepsilon+\Delta)$ yielding Eq. (60) was proposed by Coleridge (1990), although it was erroneously also suggested there that MISO are suppressed by the thermal averaging if $T \gg \omega_{c}$.

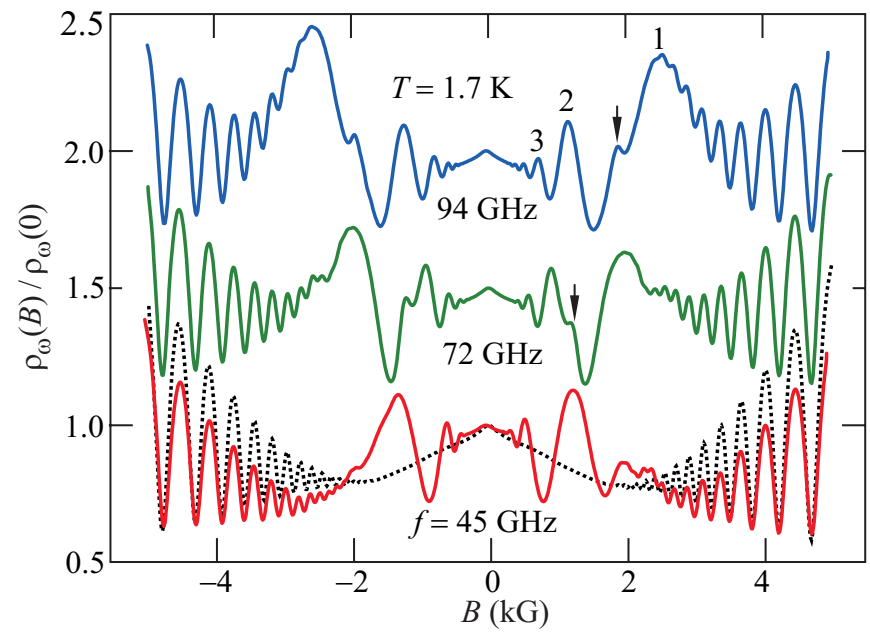

FIG. 5 Magnetoresistivity with (solid lines) and without (dashed) microwave irradiation for different frequencies $f=$ 45,72 , and $94 \mathrm{GHz}$, normalized to its value at $B=0$. The traces for different $f$ are vertically offset for clarity. Integers show the order of MIRO peaks. The arrows mark the magnetoplasmon resonance. The data were obtained at $T \simeq 1.7 \mathrm{~K}$ in a $200 \mu \mathrm{m}$ wide Hall bar sample with $n_{e} \simeq 2.0 \times 10^{11} \mathrm{~cm}^{-2}$ and $\mu \simeq 3.0 \times 10^{6} \mathrm{~cm}^{2} / \mathrm{V}$ s. Adapted from Zudov et al. (2001a).

Bykov et al. (2010b) and Andreev et al. (2011) in contactless measurements in a capacitively coupled 2DEG. MIRO have been studied not only in high-mobility singlesubband 2DEGs but in a variety of other 2D systems, including 2DEGs patterned with a triangular antidot lattice (Yuan et al., 2006), see Sec.III.C.5. two-subband 2DEGs (Bykov et al., 2010c; Wiedmann et al., 2008), see Sec.III.C.5 and hole systems based on C-doped GaAs/AlGaAs QWs (Du et al., 2004). Phenomenologically similar oscillations were recently discovered in a nondegenerate electron system on surface of liquid ${ }^{3} \mathrm{He}$ (Konstantinov and Kono, 2009), see Sec.VII.B.1.

Period and phase. The radiation-induced oscillatory part $\delta \rho_{\omega}$ of the photoresistivity $\rho_{\omega}$ at a radiation frequency $\omega$ oscillates with the ratio

$$
\epsilon_{\mathrm{ac}} \equiv \omega / \omega_{\mathrm{c}},
$$

see Fig.6b. In contrast to PIRO and HIRO (Sec.V), the MIRO period is not sensitive to the carrier density $n_{e}$. MIRO maxima $\left(\epsilon_{\mathrm{ac}}^{+}\right)$and minima $\left(\epsilon_{\mathrm{ac}}^{-}\right)$are roughly symmetrically offset from the harmonics of the $\mathrm{CR}$ at $\epsilon_{\mathrm{ac}}=n^{17}$ and occur at

$$
\epsilon_{\mathrm{ac}}^{ \pm} \simeq n \mp \varphi_{\mathrm{ac}}, n=1,2,3, \ldots .
$$

In accordance with theoretical predictions for the regime of overlapping LLs (Sec.III.B.1.a), the observations sug-

17 Early experiments Ye et al., 2001; Zudov et al., 1997, 2001a) loosely associated the low-order oscillation maxima with integer $\epsilon_{\mathrm{ac}}=n$. Now it is established that at $\epsilon_{\mathrm{ac}}=n$ there are zeroresponse nodes where the photoresistivity $\delta \rho_{\omega}=0$. 

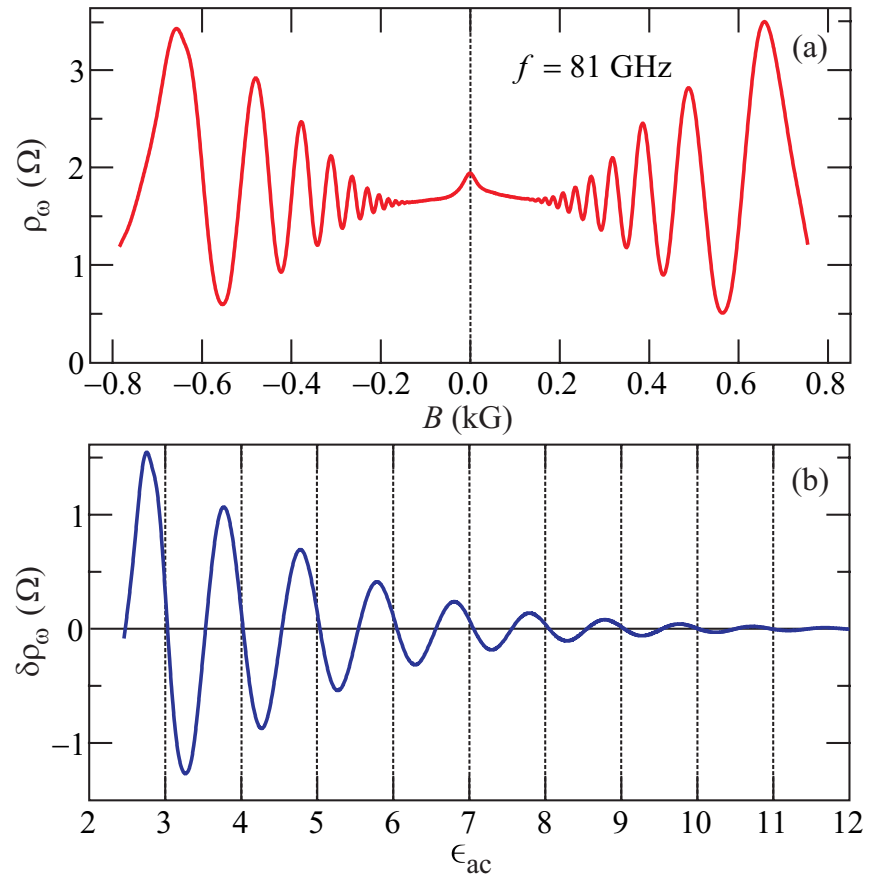

FIG. 6 (a) Magnetoresistivity oscillations with varying $B$ at fixed $f=81 \mathrm{GHz}$. (b) Microwave-induced correction $\delta \rho_{\omega}$ to the resistivity as a function of $\epsilon_{\mathrm{ac}}$, obtained by subtracting the slowly varying background. The data were obtained at $T \simeq$ $1.5 \mathrm{~K}$ in a $100 \mu \mathrm{m}$ wide Hall bar sample with $n_{e} \simeq 3.0 \times 10^{11}$ $\mathrm{cm}^{-2}$ and $\mu \simeq 1.2 \times 10^{7} \mathrm{~cm}^{2} / \mathrm{V} \mathrm{s}$.

gest that $\delta \rho_{\omega} \propto-\sin 2 \pi \epsilon_{\mathrm{ac}}$ for $\epsilon_{\mathrm{ac}} \gtrsim 2$, i.e., the phase $\varphi_{\mathrm{ac}}$ in Eq. (63) is $1 / 4$. This value of the phase was reported as universal in many experiments (Mani, 2004b, 2005, 2007a.b, 2008; Mani et al., 2010, 2009, 2004a, b, 2002, 2004c,d). Other experiments (Dai et al., 2010; Hatke et al., 2008a, b, 2011b c, d; Studenikin et al., 2005, 2007; Zhang et al., 2007a; Zudov, 2004; Zudov et al., $2006 \mathrm{a}, \mathrm{b}$ ) found that the lower-order maxima and minima are pushed toward the harmonics of the CR and are best described by $\varphi_{\text {ac }}$ which is considerably smaller than $1 / 4$. Such a phase reduction is expected in the regime of separated LLs (Dmitriev et al., 2005; Studenikin et al., 2005; Vavilov and Aleiner, 2004; Zudov, 2004), see Sec.III.B.1.a. Moreover, the phase decreases with increasing radiation power, as discussed next.

Power dependence. At sufficiently low microwave power $P$, one expects that the amplitude $A_{\omega}$ [as defined in Eq. [64)] of the oscillatory photoresistivity $\delta \rho_{\omega}$ is linear in $P$. While the linear dependence of $A_{\omega}$ on $P$ was clearly observed by Hatke et al. (2011b); Zudov et al. (2003), a number of experiments found a strongly sublinear dependence (Mani et al., 2010, 2004b; Studenikin et al., 2004; Willett et al., 2004; Ye et al., 2001). The apparently conflicting experimental reports can be reconciled by the existence of two distinct regimes of weak and strong power, characterized by the linear and sublinear scaling of $A_{\omega}$ with $P$, respectively. The experimental data in Fig. 7
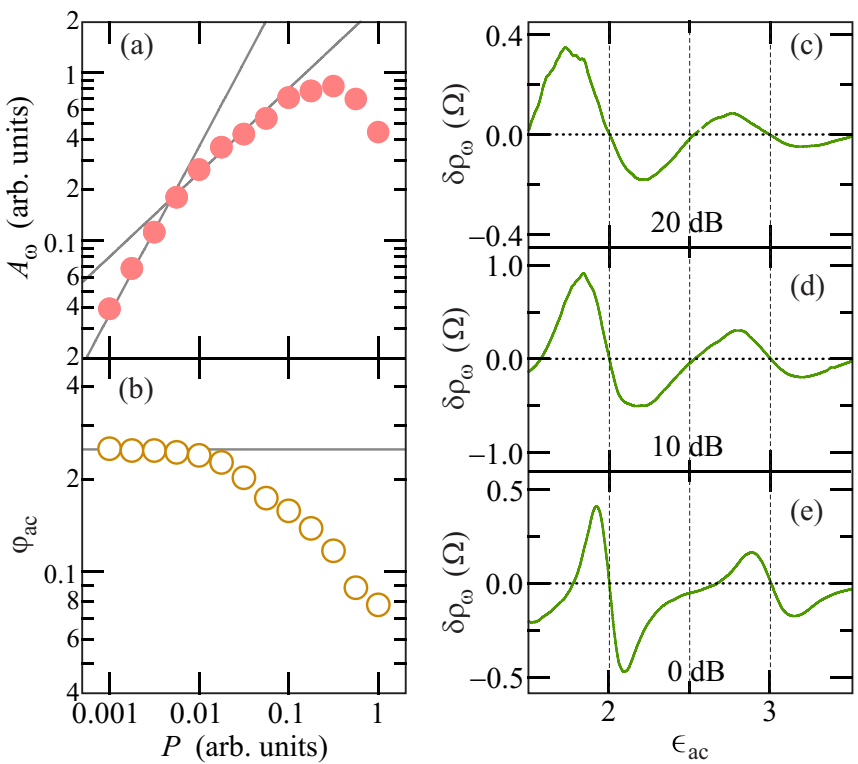

FIG. 7 (a) Amplitude $A_{\omega}$ and (b) phase $\varphi_{\text {ac }}$ measured at the second MIRO maximum vs microwave power $P$. Straight lines in part (a) represent, on the log-log scale, the linear and square-root dependences on $P$. (c)-(e) Photoresistivity $\delta \rho_{\omega}$ as a function of $\omega_{\mathrm{c}}$ at fixed $\omega / 2 \pi=33 \mathrm{GHz}$ for different $P$. The traces are labeled according to the attenuation levels. The data were obtained at $T \simeq 1.5 \mathrm{~K}$ in a $200 \mu \mathrm{m}$ wide Hall bar sample with $n_{e} \simeq 2.9 \times 10^{11} \mathrm{~cm}^{-2}$ and $\mu \simeq 2.4 \times 10^{7} \mathrm{~cm}^{2} / \mathrm{V} \mathrm{s}$. Adapted from Hatke et al. (2011a).

show that the linear dependence of $A_{\omega} \propto P$ crosses over with increasing $P$ into $A_{\omega} \propto P^{1 / 2}$, accompanied by a strong reduction of the phase $\varphi_{\mathrm{ac}}$ compared to its value $\varphi_{\mathrm{ac}}=1 / 4$ in the linear regime. Such behavior is expected for the inelastic mechanism of MIRO, see Sec.III.B.2. For the displacement mechanism, a similar effect is expected at the crossover to the multiphoton regime of the photoresponse, see Sec.VI.

Temperature dependence. Most experiments show that MIRO are best observed at temperature $T \sim 0.5-1 \mathrm{~K}$, get strongly suppressed with increasing $T$, and become almost invisible at $T \sim 4-7 \mathrm{~K}$. The overall behavior in the regime of weak oscillations, illustrated in Fig.8, is consistent with the theoretical predictions, see Fig.13b,c and the discussion in Secs. III.B.1.e III.B.1.f, and III.B.1.g [the $T$ dependence of MIRO in the regime of ZRS is discussed in Sec.IV.A. At low $B$ and $P$, the $B$ dependence of MIRO almost perfectly follows

$$
\delta \rho_{\omega}=-A_{\omega} \epsilon_{\mathrm{ac}} \sin \left(2 \pi \epsilon_{\mathrm{ac}}\right) \exp \left(-2 \pi / \omega_{\mathrm{c}} \tau_{\mathrm{q}}^{*}\right),
$$

where $A_{\omega}$ is nearly $B$-independent for $\epsilon_{\mathrm{ac}} \gtrsim 2$, see, e.g., Fig.8 8 . Such fits of the $B$-traces of MIRO at different $T$ generally reveal that both $\tau_{\mathrm{q}}^{*}$ in the factor $e^{-2 \pi / \omega_{\mathrm{c}} \tau_{\mathrm{q}}^{*}}$ and $A_{\omega}$ depend on $T$. Specifically, the exponential factor in Eq. (64) is well described by a quadratic-in- $T$ dependence of $1 / \tau_{\mathrm{q}}^{*}(T)=1 / \tau_{\mathrm{q}}+\alpha T^{2}$, see Fig. $8 \mathrm{~b}$, which is attributed to the LL broadening induced by electronelectron interactions (Sec.【II.B.1.f). A similar expo- 

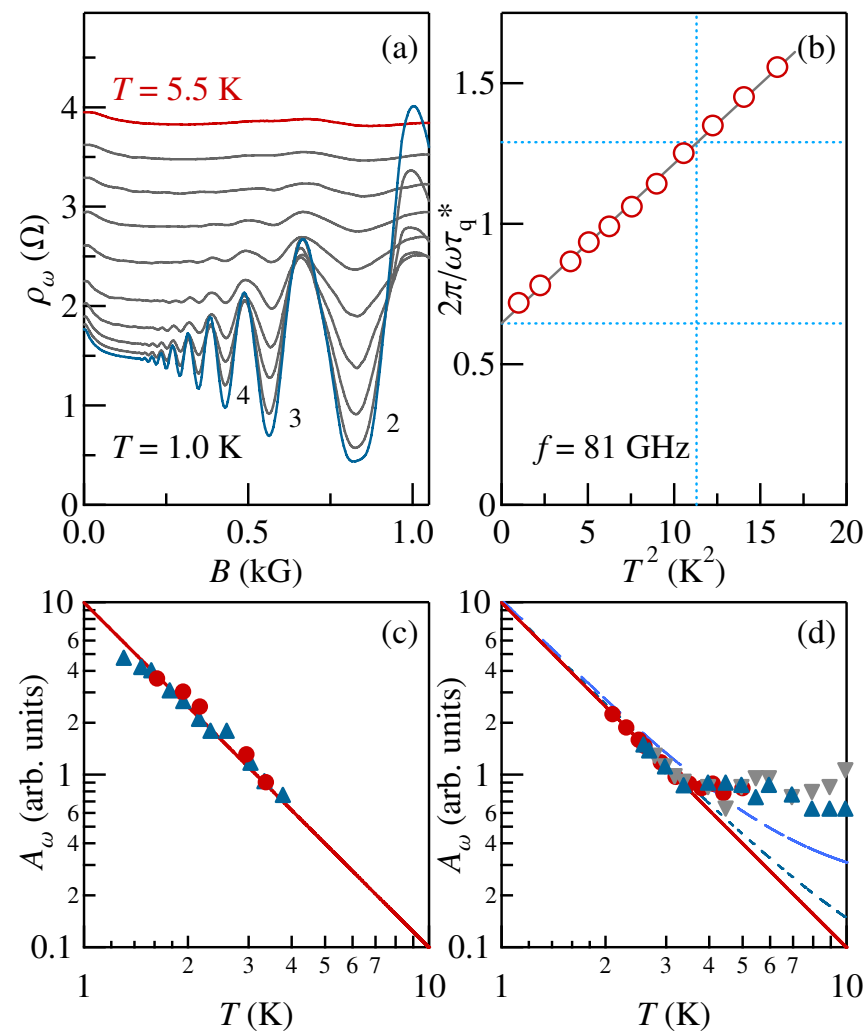

FIG. 8 (a) Evolution of MIRO with varying $T$ and (b) the $T$ dependence of $2 \pi / \omega \tau_{\mathrm{a}}^{*}$ extracted from these data by fitting them to Eq. (64) (Hatke et al., 2009c). (c)-(d) The $T$ dependence of $A_{\omega}[$ Eq. (64)] obtained by (c) Studenikin et al. (2007) and (d) Wiedmann et al. (2010b).

nential dependence on $T$ was also reported for HIRO (Hatke et al., 2009b), PIRO (Hatke et al., 2009a), and MISO (Mamani et al., 2008). Studenikin et al. (2005, 2007); Wiedmann et al. (2010b) found $A_{\omega} \propto T^{-2}$ at $T<3 \mathrm{~K}$, see Fig. 86, d, consistent with the inelastic mechanism of MIRO (Sec.III.B.1.e). The saturation of the dependence of $A_{\omega}$ on $T$ observed by Wiedmann et al. $(2010 \mathrm{~b})$ at $T>3 \mathrm{~K}$, see Fig.8 $8 \mathrm{~d}$, and the results of Hatke et al. (2009c), where $A_{\omega}$ almost independent of $T$ was reported, indicate that the displacement contribution to MIRO [which produces $A_{\omega}=\operatorname{const}(T)$ and strongly depends on the correlation properties of disorder, see Sec.III.B.1.d may remain relevant down to low $T$.

Frequency dependence. Over the past decade MIRO have been observed in a wide range of radiation frequencies, from $3 \mathrm{GHz}$ (Willett et al., 2004) to $1.5 \mathrm{THz}$ (Wirthmann et al., 2007). However, the majority of experiments employed frequencies from 30 to $150 \mathrm{GHz}$, which appears to be the optimum range for a typical 2DEG. At lower frequencies, MIRO are shifted towards weaker $B$ and are therefore suppressed by the Dingle factor. At higher frequencies, the oscillation amplitude decays as well (Studenikin et al., 2007; Tung et al., 2009; Yang et al., 2003), as illustrated in Fig.9 for the pho- toconductance measured in a Corbino disk Yang et al., 2003). At fixed both $\epsilon_{\mathrm{ac}} \geq 2$ and the microwave intensity, the observed decay is consistent with $\delta \rho_{\omega} \propto \omega^{-4}$, in agreement with Eqs. (74), (75), (92), and (99) below.

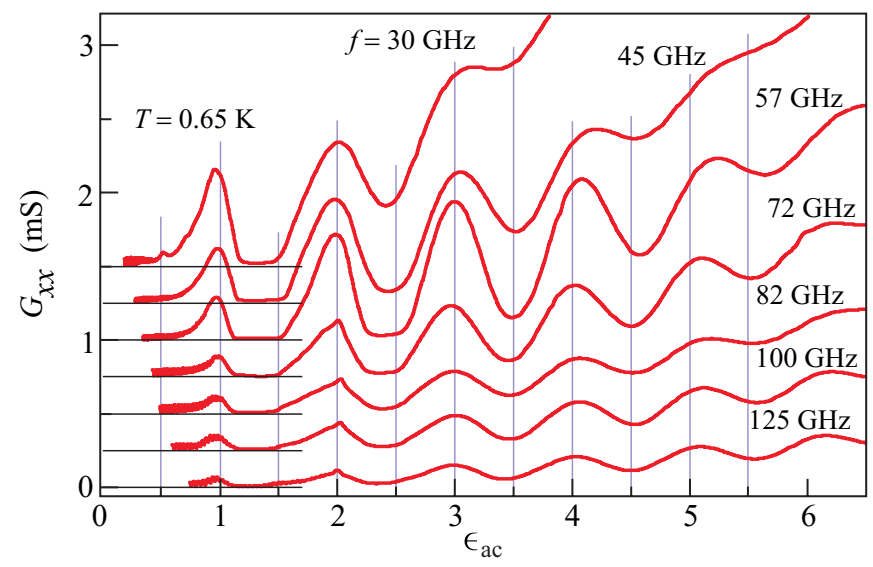

FIG. 9 Conductance $G_{x x}$ of a Corbino sample $\left(n_{e}=3.55 \times\right.$ $\left.10^{11} \mathrm{~cm}^{-2}, \mu=1.28 \times 10^{7} \mathrm{~cm}^{2} / \mathrm{V} \mathrm{s}\right)$ as a function of $\omega / \omega_{\mathrm{c}}$ for different fixed $\omega$. The traces, labeled according to $f=$ $2 \pi \omega$, are offset in steps of $0.25 \mathrm{mS}$. Adapted from Yang et al. (2003).

\section{B. Microscopic mechanisms of MIRO}

Most experimental findings concerning MIRO can be explained as a combined effect of Landau quantization and external fields either on the momentum relaxation (displacement mechanism) or on the energy distribution of electrons within disorder-broadened LLs (inelastic mechanism). Section III.B.1 describes both effects in terms of quasiclassical kinetics of the guiding centers of cyclotron orbits. Section III.B.2 deals with the main mechanism of MIRO saturation which establishes the conditions for the observation of ZRS and fixes the amplitude of a dc electric field in spontaneously formed domains. A quantum kinetic equation formalism is described in Sec.III.B.3. This formalism provides a solid foundation for the approach used in Secs. III.B.1 and III.B.2 and serves as a basic tool for the theoretical description of other nonequilibrium phenomena throughout the review. Additional quadrupole and photovoltaic contributions to MIRO (these govern, in particular, magnetooscillations of the Hall part of the conductivity) are discussed in Sec.III.B.4 Alternative mechanisms of MIRO, not related to the Landau quantization, are shortly discussed in Sec.III.B.5.

\section{Inelastic and displacement mechanisms}

Initially MIRO were attributed (Durst et al., 2003; Vavilov and Aleiner, 2004) to the displacement mechanism which accounts for spatial displacements of 
quasiclassical electron orbits due to radiation-assisted scattering off disorder. Because of Landau quantization, which leads to a periodic modulation in the DOS $\nu(\varepsilon) \simeq \nu\left(\varepsilon+\omega_{\mathrm{c}}\right)$ (Sec.II.C.1), the preferred direction of these displacements with respect to the symmetrybreaking dc field oscillates with $\omega / \omega_{\mathrm{c}}$. This results in MIRO with a phase and a period which agree with those observed in experiment. Photoconductivity oscillations governed by the displacement mechanism were in fact predicted long ago (Rvzhii, 1970; Rvzhii et al., 1986) in the limit of separated LLs and a strong dc field. This mechanism was further studied using various approaches and approximations in a number of theoretical works Anderson and Brinkman, 2003; Auerbach and Pai, 2007; Dmitriev et al., 2009b, 2007a; Durst et al., 2003; Kashuba, 2006a, b; Khodas and Vavilov, 2008; Lee and Leinaas, 2004; Lei and Liu, 2003; Park, 2004; Rvzhii et al., 2004; Rvzhii and Suris, 2003; Shi and Xie, 2003; Torres and Kunold, 2005; Vavilov and Aleiner, 2004; Volkov and Takhtamirov, 2007). Soon after the experimental observations of MIRO, Dmitriev et al. (2003) proposed that the dominant contribution to MIRO was due to the inelastic mechanism associated with radiation-induced changes in the occupation numbers of electron states. Similar ideas were discussed by Dorozhkin (2003); however, the calculation there was not directly applicable to the experimentally relevant systems, in particular, due to an unrealistic model of inelastic relaxation. Later studies confirmed that the inelastic mechanism generally dominates the observed MIRO (Dmitriev et al., 2005) at low $T$, while the displacement mechanism can be relevant at higher $T$ and only if a sufficient amount of short-range impurities is present in the system (Dmitriev et al., 2009b; Khodas and Vavilov, 2008), or else, in the limit of a strong de field (Khodas and Vavilov, 2008) or high microwave power (Dmitriev et al., 2007a).

In this section, we formulate a description of both mechanisms in terms of migration of the guiding centers of cyclotron orbits. A systematic way to obtain the same results within the quantum kinetic approach is sketched in Sec.III.B.3. Quasiclassically, each scattering event leads to a shift of the guiding center $\Delta \boldsymbol{R}_{\varphi_{1} \varphi_{2}}=$ $R_{c} \mathbf{e}_{z} \times\left(\boldsymbol{n}_{\varphi_{1}}-\boldsymbol{n}_{\varphi_{2}}\right)$, where $\boldsymbol{n}_{\varphi_{k}}=\left(\cos \varphi_{k}, \sin \varphi_{k}\right)$ with $k=1,2$ are the unit vectors in the direction of motion before and after the collision, see Fig. 10 .

For a macroscopically homogeneous 2DEG subjected to a dc electric field $\boldsymbol{E}=\mathbf{e}_{x} E$, the dissipative current

$$
j_{d}=2 \nu_{0} e \int_{-\infty}^{x} d x_{1} \int_{x}^{\infty} d x_{2}\left(W_{x_{1} \rightarrow x_{2}}-W_{x_{2} \rightarrow x_{1}}\right)
$$

is expressed in terms of the probabilities $W_{x_{1} \rightarrow x_{2}}$ of the guiding-center shifts $x_{1} \rightarrow x_{2}$ along the $\mathbf{e}_{x}$ axis $\Delta X_{\varphi_{1} \varphi_{2}}=\mathbf{e}_{x} \cdot \Delta \boldsymbol{R}_{\varphi_{1} \varphi_{2}}=R_{c}\left(\sin \varphi_{1}-\sin \varphi_{2}\right)$. The probabilities are given by the integrals over the initial and
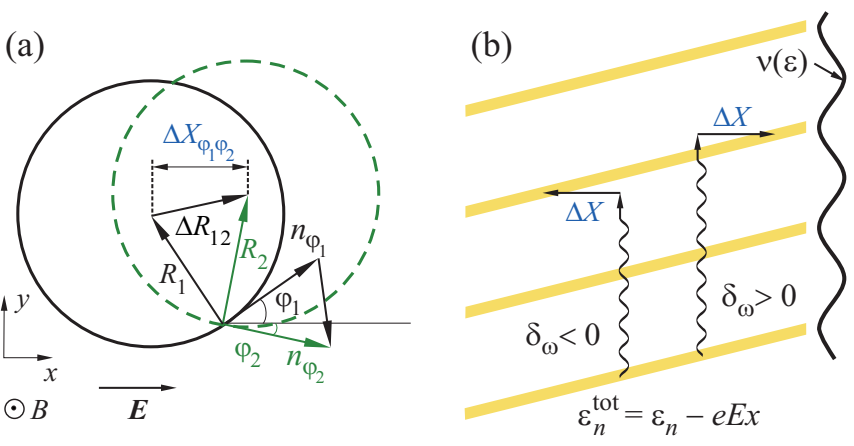

FIG. 10 (a) Shift of the guiding center of a cyclotron orbit due to quasielastic scattering off disorder. (b) Schematics of the correlations between the direction of the cyclotron-orbit shifts $\Delta X$ in the process of photon absorption and the sign of the detuning $\delta_{\omega}=\omega / \omega_{\mathrm{c}}-2$ for the second harmonic of the CR. The yellow stripes mark the DOS maxima $\varepsilon_{n}=(n+1 / 2) \omega_{\mathrm{c}}$ in LLs tilted by a dc field.

final energies in the scattering event

$$
\begin{aligned}
& W_{x_{1} \rightarrow x_{2}}=\left\langle\int d \varepsilon_{1} \int d \varepsilon_{2} \mathcal{M}_{\varepsilon_{1} \varepsilon_{2}} \delta\left(x_{1}-x_{2}+\Delta X_{\varphi_{1} \varphi_{2}}\right)\right. \\
& \left.\times\left[\Gamma_{\varphi_{1} \varphi_{2}}^{(\mathrm{el})} \delta\left(\Delta \varepsilon_{12}^{\mathrm{tot}}\right)+\Gamma_{\varphi_{1} \varphi_{2}}^{(\mathrm{ph})} \sum_{ \pm} \delta\left(\Delta \varepsilon_{12}^{\mathrm{tot}} \pm \omega\right)\right]\right\rangle_{\varphi_{1} \varphi_{2}},
\end{aligned}
$$

where the angular brackets denote averaging over the initial and final angles $\varphi_{1,2}$. The delta functions in the square brackets express conservation of the total electron energy $\Delta \varepsilon_{12}^{\text {tot }}=\varepsilon_{1}-\varepsilon_{2}+e E \Delta X_{\varphi_{1} \varphi_{2}}=0$ in the elastic channel $\left(\propto \Gamma_{\varphi_{1} \varphi_{2}}^{(\mathrm{el})}\right)$ and its change by $\pm \omega$ in the photon-assisted scattering channel $\left(\propto \Gamma_{\varphi_{1} \varphi_{2}}^{(\mathrm{ph})}\right)$. Quantum magnetooscillations originate from the factor

$$
\mathcal{M}_{\varepsilon \varepsilon^{\prime}}=\tilde{\nu}_{\varepsilon} \tilde{\nu}_{\varepsilon^{\prime}} f_{\varepsilon}\left(1-f_{\varepsilon^{\prime}}\right)
$$

where $\tilde{\nu}_{\varepsilon}=\nu(\varepsilon) / \nu_{0}$ with $\nu_{0}=m / 2 \pi$ is the dimensionless DOS in disorder-broadened LLs and $f_{\varepsilon}$ is the nonequilibrium distribution function in the steady state. In the homogeneous case, both $\tilde{\nu}_{\varepsilon}$ and $f_{\varepsilon}$ are functions of the local kinetic energy $\varepsilon$. Since the disorder-induced broadening of LLs is determined by $\tau_{\mathrm{q}} \ll \tau$ (Sec. II.C.1), the effect of the external fields on $\tilde{\nu}_{\varepsilon}$ is negligible in the relevant range of the $\mathrm{dc}$ and microwave field strength. By contrast, the modification of the distribution function is crucially important. The nonequilibrium occupation of electron states is governed by the kinetic equation

$$
\begin{aligned}
& \left\langle\tilde{\nu}_{\varepsilon}^{-1} \Gamma_{\varphi \varphi^{\prime}}^{(\mathrm{ph})} \sum_{ \pm}\left(\mathcal{M}_{\varepsilon \varepsilon^{\prime} \pm \omega}-\mathcal{M}_{\varepsilon^{\prime} \pm \omega \varepsilon}\right)\right\rangle_{\varphi \varphi^{\prime}} \\
& +\left\langle\tilde{\nu}_{\varepsilon}^{-1} \Gamma_{\varphi \varphi^{\prime}}^{(\mathrm{el})}\left(\mathcal{M}_{\varepsilon \varepsilon^{\prime}}-\mathcal{M}_{\varepsilon^{\prime} \varepsilon}\right)\right\rangle_{\varphi \varphi^{\prime}}=\operatorname{St}_{\mathrm{in}}\left\{f_{\varepsilon}\right\},
\end{aligned}
$$

where $\varepsilon^{\prime}=\varepsilon+e E \Delta X_{\varphi \varphi^{\prime}}$. To close the set of equations, the type of inelastic scattering and the expressions for the rates $\Gamma^{(\mathrm{el})}$ and $\Gamma^{(\mathrm{ph})}$ need to be specified. The calculation in Sec.III.B.1.e below shows that the inelastic scattering 
integral can be approximated in the form

$$
\operatorname{St}_{\text {in }}\left\{f_{\varepsilon}\right\}=\left(f_{\varepsilon}^{T}-f_{\varepsilon}\right) / \tau_{\text {in }},
$$

which describes thermalization to the local Fermi distribution $f_{\varepsilon}^{T}$ with the effective rate $1 / \tau_{\text {in }}$. The scattering rates $\Gamma_{\varphi \varphi^{\prime}}^{(\mathrm{ph})}$ and $\Gamma_{\varphi \varphi^{\prime}}^{(\mathrm{el})}$ for transitions $\varphi \rightarrow \varphi^{\prime}$ are given by (Khodas and Vavilov, 2008)

$$
\Gamma_{\varphi \varphi^{\prime}}^{(\mathrm{ph})}=\frac{P_{\varphi+\varphi^{\prime}}}{2 \tau_{\varphi-\varphi^{\prime}}} \sin ^{2} \frac{\varphi-\varphi^{\prime}}{2}, \quad \Gamma_{\varphi \varphi^{\prime}}^{(\mathrm{el})}=\frac{1}{\tau_{\varphi-\varphi^{\prime}}}-2 \Gamma_{\varphi \varphi^{\prime}}^{(\mathrm{ph})} .
$$

The last term in $\Gamma_{\varphi \varphi^{\prime}}^{(\mathrm{el})}$ describes the microwave-induced modification of the elastic scattering. For the isotropic 2DEG, the disorder-induced scattering rate $\tau_{\varphi_{1}-\varphi_{2}}^{-1}$ is expressible in the most general case as a series in angular harmonics

$$
\tau_{\varphi_{1}-\varphi_{2}}^{-1}=\sum_{n=-\infty}^{\infty} \tau_{n}^{-1} e^{i n\left(\varphi_{1}-\varphi_{2}\right)}, \quad \tau_{n}=\tau_{-n} .
$$

For the two-component disorder model, the coefficients $\tau_{n}$ are discussed in Sec.III.B.1.d For the microwave field (screened by the 2DEG, see Sec.III.B.1.c) of the form

$$
\boldsymbol{E}_{\omega}(t)=E_{\omega} \sum_{ \pm} \operatorname{Re}\left(s_{ \pm} \mathbf{e}_{ \pm} e^{i \omega t}\right)
$$

where $2^{1 / 2} \mathbf{e}_{ \pm}=\mathbf{e}_{x} \pm i \mathbf{e}_{y}$ and $\left(s_{+}, s_{-}\right)$is the complex vector of unit length which determines the polarization of the field, the dimensionless power $P_{\theta}$ in Eq. (70) is written as

$$
\begin{aligned}
& P_{\theta}=\mathcal{P}-2 \operatorname{Re}\left(\mathcal{E}_{+} \mathcal{E}_{-}^{*} e^{i \theta}\right), \\
& \mathcal{P}=\left|\mathcal{E}_{+}\right|^{2}+\left|\mathcal{E}_{-}\right|^{2}, \\
& \mathcal{E}_{ \pm}=s_{ \pm} e v_{F} E_{\omega} \omega^{-1}\left(\omega \pm \omega_{c}\right)^{-1} .
\end{aligned}
$$

The golden-rule approach formulated above describes a rich variety of phenomena in strong dc and microwave fields. We first discuss it in the case of MIRO and calculate the linear direct current at high temperature, namely for $2 \pi^{2} T / \omega_{\mathrm{c}} \gg 1$, to order $E E_{\omega}^{2}$. The current reads

$$
j_{d}=\sigma_{\mathrm{D}}\left\langle\tilde{\nu}_{\varepsilon}^{2}\right\rangle_{\varepsilon} E+\sigma^{\mathrm{dis}} E+\sigma^{\mathrm{in}} E .
$$

Here the first term-describing the linear dark conductivity - follows directly from Eq. (39) and $\langle\ldots\rangle_{\varepsilon}$ denotes the energy averaging over the period $\omega_{\mathrm{c}}$. The two other terms are microwave-induced corrections, at this order in the external fields completely independent of each other. The displacement contribution, proportional to $\sigma^{\text {dis }}$, originates from the photon-assisted displacements in Eqs. (65), (66) if one substitutes the equilibrium function $f_{\varepsilon}^{(T)}$ for $f_{\varepsilon}$. In the limit $2 \pi^{2} T / \omega_{\mathrm{c}} \gg 1$, a straightforward calculation yields

$$
\begin{aligned}
\sigma^{\mathrm{dis}} & =\sigma_{\mathrm{D}} \frac{\tau}{4 \tau_{\star}}\left[\mathcal{P}-\operatorname{Re}\left(\mathcal{E}_{+} \mathcal{E}_{-}^{*}\right)\right]\left(\mathcal{R}_{1}-\mathcal{R}_{3}\right), \\
\mathcal{R}_{1} & =\omega \partial_{\omega}\left\langle\tilde{\nu}_{\varepsilon} \tilde{\nu}_{\varepsilon+\omega}\right\rangle_{\varepsilon} \\
\mathcal{R}_{3} & =\left\langle\tilde{\nu}_{\varepsilon}^{2}-\tilde{\nu}_{\varepsilon} \tilde{\nu}_{\varepsilon+\omega}\right\rangle_{\varepsilon}
\end{aligned}
$$

where $\tau_{\star}^{-1}$ is expressed in terms of the partial contributions (71) to the disorder-induced scattering rate as follows (the angle brackets denote averaging over $\theta$ ):

$$
\tau_{\star}^{-1}=2\left\langle\tau_{\theta}^{-1}(1-\cos \theta)^{2}\right\rangle_{\theta}=3 \tau_{0}^{-1}-4 \tau_{1}^{-1}+\tau_{2}^{-1} .
$$

The inelastic contribution to $j_{d}$, proportional to $\sigma^{\text {in }}$, accounts for the microwave-induced change $\delta f_{\varepsilon}=f_{\varepsilon}-f_{\varepsilon}^{T}$ of the distribution function. For $E \rightarrow 0$, the last term in Eq. (68) vanishes and $\varepsilon=\varepsilon^{\prime}$. To first order in $E_{\omega}^{2}$,

$$
\begin{aligned}
\delta f_{\varepsilon} & =\mathcal{P} \frac{\tau_{\text {in }}}{4 \tau} \sum_{ \pm}\left(f_{\varepsilon \pm \omega}^{T}-f_{\varepsilon}^{T}\right) \tilde{\nu}_{\varepsilon \pm \omega} \\
& \simeq \mathcal{P} \frac{\omega \tau_{\text {in }}}{4 \tau}\left(\tilde{\nu}_{\varepsilon+\omega}-\tilde{\nu}_{\varepsilon-\omega}\right) \partial_{\varepsilon} f_{\varepsilon}^{T} .
\end{aligned}
$$

The approximation in the last line is valid for $T \gg \omega$.

At order $E E_{\omega}^{2}$ in $j_{d}$, the correction (81) of order $E_{\omega}^{2}$ should be substituted into Eqs. (65)-(67) taken at first order in $E$, which gives

$$
\begin{aligned}
& \sigma^{\text {in }}=-\sigma_{\mathrm{D}} \int d \varepsilon \tilde{\nu}_{\varepsilon}^{2} \partial_{\varepsilon} \delta f_{\varepsilon}=\sigma_{\mathrm{D}} \frac{\tau_{\text {in }}}{4 \tau} \mathcal{P} \mathcal{R}_{2}, \\
& \mathcal{R}_{2}=\omega \partial_{\omega}\left\langle\tilde{\nu}_{\varepsilon}^{2}\left(\tilde{\nu}_{\varepsilon+\omega}+\tilde{\nu}_{\varepsilon-\omega}\right)\right\rangle_{\varepsilon} .
\end{aligned}
$$

Next we summarize the most important properties of MIRO that follow from the above results.

a. Period and phase. These are determined by the factors $\mathcal{R}_{i}$ defined in Eqs. (78), (79), and (83). In the limit of overlapping LLs, the DOS is given by Eq. (31), $\tilde{\nu}(\varepsilon)=$ $1-2 \delta \cos \left(2 \pi \varepsilon / \omega_{c}\right)$ with $\delta=\exp \left(-\pi / \omega_{c} \tau_{\mathrm{q}}\right) \ll 1$, so that

$$
\begin{aligned}
& \mathcal{R}_{1}=\mathcal{R}_{2} / 4=-4 \delta^{2} \frac{\pi \omega}{\omega_{\mathrm{c}}} \sin \frac{2 \pi \omega}{\omega_{\mathrm{c}}}, \\
& \mathcal{R}_{3}=4 \delta^{2} \sin ^{2} \frac{\pi \omega}{\omega_{\mathrm{c}}} .
\end{aligned}
$$

We see that, for both the displacement and inelastic mechanisms, MIRO are proportional to $\sin \left(2 \pi \omega / \omega_{\mathrm{c}}\right)$ with a negative coefficient in front of it in accord with the experimental findings (for the displacement mechanism, the phase of MIRO also agrees with the observed one for large $\pi \omega / \omega_{c}$, when $\mathcal{R}_{3}$ can be neglected compared to $\left.\mathcal{R}_{1}\right)$.

The phase of the oscillations can be qualitatively understood as follows. Owing to the oscillatory behavior of $\tilde{\nu}_{\varepsilon} \tilde{\nu}_{\varepsilon \pm \omega+e E \Delta X}$, which enters Eq. (66) via the factor (67), the average displacement of a cyclotron orbit $\Delta X$ (Fig. 10b) is positive (negative) for a small positive (negative) detuning $\delta_{\omega}=\omega / \omega_{c}-N$ from the $N$ th harmonic of the CR. The uphill drift for $\delta_{\omega}>0$ produces a contribution to the dissipative current (65) which is directed against the electric field, i.e., $\sigma^{\text {dis }}<0$. For the inelastic mechanism, a small positive detuning $\delta_{\omega}$ increases (decreases) the occupation of states lying right above (below) the maxima of $\tilde{\nu}(\varepsilon)$ compared to equilibrium (Fig. 11), while the negative detuning leads to the opposite sign of the nonequlibirum correction to the distribution function. The phase difference between the oscillations of $\nu(\varepsilon)$ and those of $\partial_{\varepsilon} \delta f_{\varepsilon}$ in Eq. (82) is such 

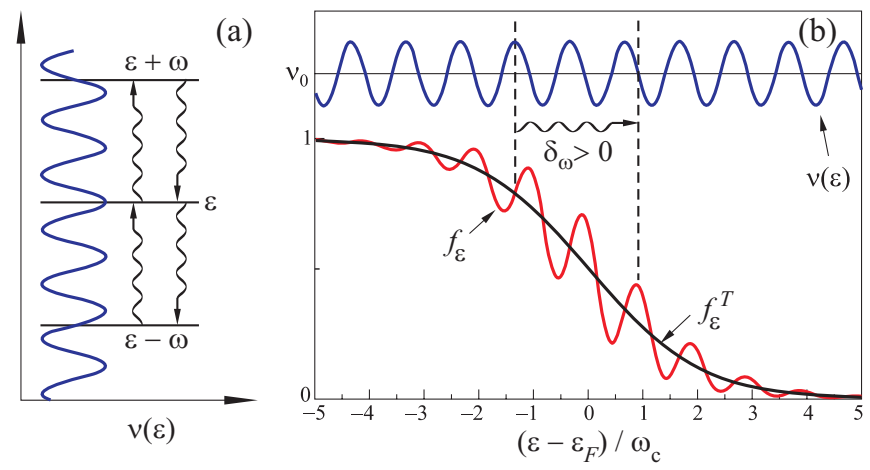

FIG. 11 (a) Emission/absorption of microwave quanta for the detuning $\delta_{\omega}=\omega / \omega_{\mathrm{c}}-2=1 / 4$ and (b) the DOS $\nu(\varepsilon)$ and the resulting oscillatory distribution function $f_{\varepsilon}$ for $T=$ $\omega_{\mathrm{c}}$, compared to the thermal distribution $f_{\varepsilon}^{T}$. Adapted from Dmitriev et al. (2005).

that the sign of $-\partial_{\varepsilon} \delta f_{\varepsilon}$ at the maxima of $\tilde{\nu}(\varepsilon)$ coincides with the sign of $\sigma^{\text {in }}$ (Fig. 11), i.e., $\delta_{\omega}>0$ yields $\sigma^{\text {in }}<0$.

In the limit of separated LLs, $\omega_{\mathrm{c}} \tau_{\mathrm{q}} \gg 1$, the DOS is a sequence of semicircles (28) of width $2 \Gamma=$ $2\left(2 \omega_{\mathrm{c}} / \pi \tau_{\mathrm{q}}\right)^{1 / 2} \ll \omega_{\text {c }}$, i.e., $\tilde{\nu}(\varepsilon)=\tau_{\mathrm{q}} \operatorname{Re} \sqrt{\Gamma^{2}-(\delta \varepsilon)^{2}}$, where $\delta \varepsilon$ is the detuning from the center of the nearest LL. In this limit, one has

$$
\begin{aligned}
& \mathcal{R}_{1}=\mathcal{R}_{0} \frac{\omega}{\Gamma} \sum_{n} \operatorname{sgn}\left(\Omega_{n}\right) \mathcal{H}_{2}\left(\left|\Omega_{n}\right|\right) \\
& \mathcal{R}_{2}=-\mathcal{R}_{0} \frac{4 \omega \omega_{\mathrm{c}}}{\Gamma^{2}} \sum_{n} \operatorname{sgn}\left(\Omega_{n}\right) \Phi_{2}\left(\left|\Omega_{n}\right|\right), \\
& \mathcal{R}_{3}=\mathcal{R}_{0}\left[1-\sum_{n} \mathcal{H}_{1}\left(\left|\Omega_{n}\right|\right)\right]
\end{aligned}
$$

where $\mathcal{R}_{0}$ [which also enters Eq. (76)] reads

$$
\mathcal{R}_{0} \equiv\left\langle\tilde{\nu}_{\varepsilon}^{2}\right\rangle_{\varepsilon}=16 \omega_{\mathrm{c}} / 3 \pi^{2} \Gamma
$$

The parameterless functions of $\Omega_{n}=\left(\omega-n \omega_{\mathrm{c}}\right) / \Gamma$ are nonzero at $0<\left|\Omega_{n}\right|<2$, where they are given by

$$
\begin{aligned}
\mathcal{H}_{1}(x) & =(2+x)\left[\left(4+x^{2}\right) E(Y)-4 x K(Y)\right] / 8, \\
\mathcal{H}_{2}(x) & =3 x[(2+x) E(Y)-4 K(Y)] / 8, \\
4 \pi \Phi_{2}(x) & =3 x \arccos (x-1)-x(1+x) \sqrt{x(2-x)} .
\end{aligned}
$$

Here $Y=(2-x)^{2} /(2+x)^{2}$ and the functions $E$ and $K$ are the complete elliptic integrals of the first and second kind, respectively [see (Dmitriev et al., 2007b) for a graphical representation of the functions (89)-(91)]. A distinctive feature of the photoresponse in separated LLs is the presence of windows in $\omega_{\mathrm{c}}$ within which $\left|\omega-n \omega_{\mathrm{c}}\right|>2 \Gamma$ for any integer $n$ and hence both intra- and inter-LL singlephoton transitions are impossible, i.e., $\tilde{\nu}_{\varepsilon} \tilde{\nu}_{\varepsilon+\omega}=0$ for any $\varepsilon$. The resulting gaps in the dependence of the photoresponse on $B$ for a given radiation frequency were observed by Dorozhkin et al. (2005), see Fig.12] At sufficiently high microwave power, the photoresponse in the gaps becomes visible due to multiphoton effects (Sec.VI).
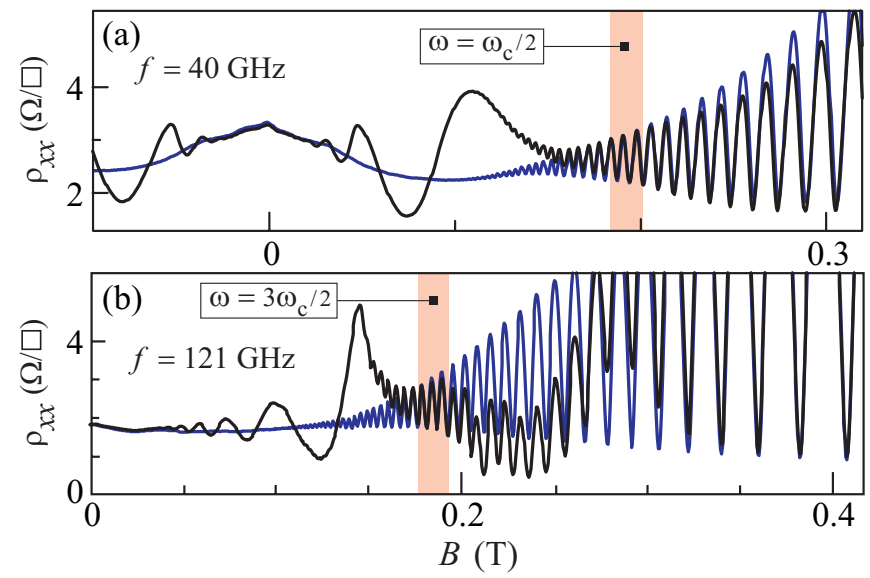

FIG. 12 Magnetoresistivity at a radiation frequency of (a) $40 \mathrm{GHz}$ and (b) $121 \mathrm{GHz}$ as a function of $B$, compared to the magnetoresistivity in the absence of radiation. The shaded boxes around the points at which (a) $\omega=\omega_{\mathrm{c}} / 2$ and (b) $\omega=3 \omega_{\mathrm{c}} / 2$ mark the range of $B$ within which the magnetoresistivity with and without radiation coincide. Adapted from Dorozhkin et al. (2005).

b. Polarization dependence. While the phase of MIRO in $\sigma^{\text {dis }}$ and $\sigma^{\text {in }}$ is essentially the same, their polarization and temperature dependences are qualitatively different. For linear polarization with $s_{ \pm}=e^{\mp i \psi} / \sqrt{2}$ characterized by the angle $\psi$ between the directions of the microwave and dc fields, $\sigma^{\text {dis }}$ contains the anisotropic term $\operatorname{Re}\left(\mathcal{E}_{+} \mathcal{E}_{-}^{*}\right) \propto 2 \operatorname{Re} s_{+}^{2}=\cos 2 \psi$, while $\sigma^{\text {in }} \propto \mathcal{P}$ does not depend on $\psi$. The polarization dependence of $\sigma^{\text {dis }}$ is illustrated in Fig. 13 in Vavilov and Aleiner (2004). Experimental results on the polarization dependence are discussed in Sec.VII.A.6.

c. Screening of the microwave field. The dimensionless field $\mathcal{E}_{ \pm}$and the polarization parameters $s_{ \pm}$in Eq. (75) represent the strength and the polarization of the total (screened) microwave field. As discussed in Sec.II.C.3 (see also Sec.VII.A.6), in high mobility samples the active circular component of the incoming electromagnetic wave is strongly suppressed near the CR due to a nearly complete reflection. Expressed in terms of the field $E_{\omega}^{(0)}$ and the polarization $s_{ \pm}^{(0)}$ of the incoming wave, Eq. (75) acquires the form

$$
\mathcal{E}_{ \pm}=\frac{s_{ \pm}^{(0)} e v_{F} E_{\omega}^{(0)}}{\omega\left(\omega \pm \omega_{\mathrm{c}}+i \gamma\right)}
$$

where $\gamma$ [Eq. (57), see also footnote11] is assumed to be much larger than $\tau^{-1}$. Since in typical experiments $\gamma$ is of order $\omega \gg \tau^{-1}$, the CR in $\mathcal{P}$ [Eq. (74)] is strongly broadened by the screening. Note also that the polarization of the screened field is generally different from that of the field in the incident wave and depends on $B$. 
d. Sensitivity to different types of disorder. The displacement contribution $\sigma^{\text {dis }}$ is highly sensitive to the details of the disorder potential Auerbach and Pail, 2007; Khodas and Vavilov, 2008), which are difficult to extract from standard transport measurements. In the mixeddisorder model (Sec. II.A), believed to provide an adequate description of high mobility 2DEGs, the partial contributions (71) to the scattering rate are given by

$$
\frac{1}{\tau_{n}}=\frac{\delta_{n 0}}{\tau_{\mathrm{sh}}}+\frac{1}{\tau_{\mathrm{q}, \mathrm{sm}}} \frac{1}{1+\chi n^{2}},
$$

where the first term describes scattering off the shortrange component of the random potential (modeled here as white-noise disorder) and the second term describes small-angle scattering off the smooth random potential created by remote donors. The characteristic scattering angle in the latter case is $\sqrt{\chi}=\left(2 k_{F} d\right)^{-1} \ll 1$. Since in high mobility structures the quantum scattering rate $\tau_{\mathrm{q}}^{-1}$, given by $\tau_{0}^{-1}$, is much larger than the transport scattering rate $\tau^{-1}=\tau_{0}^{-1}-\tau_{1}^{-1}$, the quantum scattering rate is dominated by the contribution of the long-range component: $\tau_{\mathrm{q}, \mathrm{sm}}^{-1} \gg \tau_{\mathrm{sh}}^{-1}$. That is, the LL broadening is largely determined by the smooth disorder, whereas the relative weight of the short- and long-range components in $1 / \tau \simeq 1 / \tau_{\mathrm{sh}}+\chi / \tau_{\mathrm{q}, \mathrm{sm}}$ may be arbitrary. Moreover, the displacement contribution $\sigma^{\text {dis }}$ is proportional to the rate $\tau_{\star}^{-1}$ given by Eq. (80) (Khodas and Vavilov, 2008),

$$
\frac{1}{\tau_{\star}} \simeq \frac{3}{\tau_{\mathrm{sh}}}+\frac{12 \chi^{2}}{\tau_{\mathrm{q}, \mathrm{sm}}},
$$

which contains one more power of the small parameter $\chi$ in front of $\tau_{\mathrm{q}, \mathrm{sm}}^{-1}$ compared to $\tau^{-1}$ (Vavilov and Aleiner, 2004). As a result, even a small amount of short-range scatterers may give the main contribution to $\sigma^{\text {dis }}$, as illustrated in Fig. 13 a.
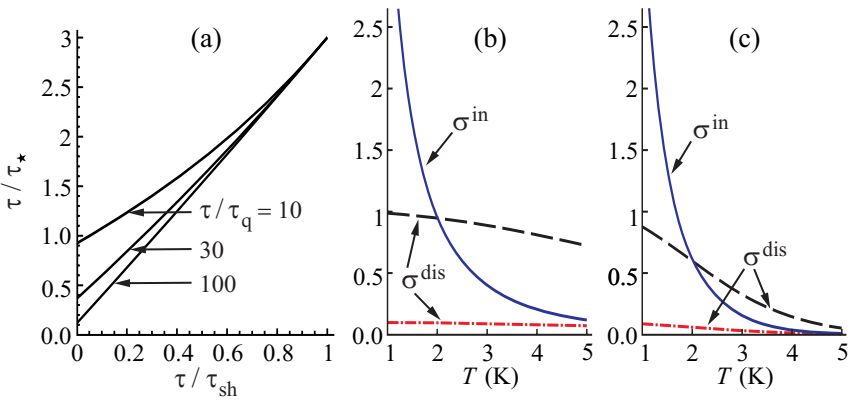

FIG. 13 (a) Dependence of $\tau / \tau_{\star}$ on the relative weight $\tau / \tau_{\text {sh }}$ of the short-range component of disorder in the transport scattering rate for fixed $\tau / \tau_{\mathrm{q}}=100,30$, and 10. (b),(c) Dependence of $\sigma^{\text {in }}$ and $\sigma^{\text {dis }}$ (in arbitrary units) on $T$ for (b) $T_{\mathrm{q}}=10 \mathrm{~K}$ and (c) $T_{\mathrm{q}}=3 \mathrm{~K}$. The curves for $\sigma^{\text {in }}$ are calculated for $\tau_{\text {in }}=2 \tau$ at $T=1 \mathrm{~K}$; the dashed curves for $\sigma^{\text {dis }}$-for strong short-range disorder with $\tau / 2 \tau_{\star}=1, \tau / \tau_{\text {sh }}=0.6$, and

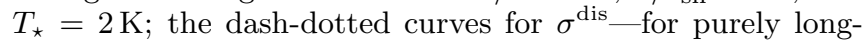
range disorder with $\tau / 2 \tau_{\star}=0.1, \tau / \tau_{\mathrm{q}}=50$, and $T_{\star} \simeq 6 \mathrm{~K}$.) Adapted from Dmitriev et al. (2009b). e. Temperature dependence. The displacement contribution [Eq. (77)] is $T$-independent (apart from the $T$ dependence of the DOS, which becomes relevant at high $T$ for both mechanisms, see Sec.III.B.1.f), while the inelastic contribution [Eq. (82)], proportional to the inelastic scattering time $\tau_{\text {in }}$, grows with lowering $T$. A detailed analysis of the inelastic relaxation was performed by Dmitriev et al. (2005). It was found that, for relevant $T$, the amplitude of the oscillations in the distribution function is controlled by electron-electron collisions. In overlapping LLs, the ansatz $f=f_{\varepsilon}^{T}+\varphi(\varepsilon) \partial_{\varepsilon} f_{\varepsilon}^{T}$ with periodic $\varphi(\varepsilon)=\varphi\left(\varepsilon+\omega_{\mathrm{c}}\right)$ reduces the linearized collision integral (68) to

$$
\begin{aligned}
\operatorname{St}_{\text {in }}\{f\} & =-\frac{\pi^{2} T^{2}+\varepsilon^{2}}{2} \frac{\partial f_{\varepsilon}^{T}}{\partial \varepsilon}\langle A(E)[\varphi(\varepsilon)-\varphi(\varepsilon+E) \\
& \left.\left.+\varphi\left(\varepsilon^{\prime}\right)-\varphi\left(\varepsilon^{\prime}-E\right)\right]\right\rangle_{\varepsilon^{\prime} E},
\end{aligned}
$$

where the angular brackets denote averaging over $\varepsilon^{\prime}$ and $E$ within the period $\omega_{c}$. For a harmonic modulation of the distribution function with $\varphi(\varepsilon) \propto \cos \left(2 \pi \varepsilon / \omega_{c}+\theta\right)$, the collision integral then acquires the form $\mathrm{St}_{\text {in }} \propto$ $\left\langle A(E)\left[1-\cos \left(2 \pi E / \omega_{c}\right)\right]\right\rangle_{E}$, where the last factor strongly suppresses the relevant energy-relaxation rate compared to the out-scattering rate. As shown by Dmitriev et al. (2005), the relaxation time approximation is justified for this collision integral and the inelastic scattering time that enters Eq. 82 is given in the relevant domain $\omega_{\mathrm{c}} \ll T \ll \omega_{\mathrm{c}}\left(\omega_{\mathrm{c}} \tau\right)^{1 / 2}$ by

$$
\tau_{\text {in }}=\int \frac{d \varepsilon}{2 \omega} \tau_{\mathrm{ee}}(\varepsilon, T)\left(f_{\varepsilon-\omega}^{T}-f_{\varepsilon+\omega}^{T}\right),
$$

where

$$
\tau_{e e}^{-1}(\varepsilon, T)=\frac{\pi^{2} T^{2}+\varepsilon^{2}}{4 \pi \varepsilon_{F}} \ln \frac{2 v_{F} / a_{B}}{\omega_{c}\left(\omega_{c} \tau\right)^{1 / 2}} .
$$

For $T \gg \omega$, Eq. (96) gives $\tau_{\text {in }} \simeq 0.822 \tau_{e e}(0, T) \propto$ $T^{-2}$ (Fig.13b,c), while for $T \ll \omega$ the result is $\tau_{\text {in }}=$ $\left(\pi^{2} T / 2 \omega\right) \tau_{\text {ee }}(0, T)$. It follows that the $T^{-2}$ scaling of $\sigma^{\text {in }}$ for $T \gg \omega$ crosses over to a $T^{-1}$ scaling for $T \ll \omega$. In separated LLs, the relaxation time approximation is not accurate parametrically; still, for an estimate - up to a factor of order unity - one can use Eq. (82) with $\tau_{\text {in }} \sim\left(\Gamma / \omega_{\mathrm{c}}\right) \tau_{e e}(0, T)$.

f. Exponential $B$ - and T-damping. According to Eqs. (77), (82), (84), the Dingle factor squared, $\delta^{2}=\exp \left(-2 \pi / \omega_{\mathrm{c}} \tau_{\mathrm{q}}\right)$, determines the damping of the MIRO in the limit of overlapping LLs for both the displacement and inelastic mechanisms. This same damping factor also describes the oscillations of the absorption coefficient for $T \gg \omega_{c}$ (Sec. II.C.3) and, as will be seen in Sec. V. HIRO and PIRO. Experimental data for these types of oscillations measured in the same sample support this prediction. On the other hand, the $\mathrm{SdH}$ measurements - if the data are fitted 
by the damping factor $\delta$-systematically yield shorter $\tau_{\mathrm{q}}^{(\mathrm{SdH})}<\tau_{\mathrm{q}}$. As discussed in Sec.II.C.2 the reason for the enhanced damping is that the $\mathrm{SdH}$ oscillations are sensitive to small, with an amplitude of the order of $\omega_{c}$, macroscopic inhomogeneities of the chemical potential, whereas the magnetooscillations that survive at $T \gg \omega_{\mathrm{c}}$ are robust with respect to them.

The experimentally observed MIRO (Sec.III.A) show at sufficiently high $T$ an exponential suppression of the oscillation amplitude as $T$ is increased (Hatke et al., 2009c; Wiedmann et al., 2010b). This effect can be explained in terms of a $T$-dependent renormalization of the DOS by electron-electron interactions (Chaplik, 1971; Dmitriev et al., 2009b; Ryzhii et al., 2004). Specifically, the quantum scattering rate $\tau_{\mathrm{q}}^{-1}$ that enters the DOS $\tilde{\nu}(\varepsilon)=1-2 \delta \cos \left(2 \pi \varepsilon / \omega_{\mathrm{c}}\right)$ via the Dingle factor $\delta$ should be substituted by

$$
\tilde{\tau}_{\mathrm{q}}^{-1}=\tau_{\mathrm{q}}^{-1}+\tau_{\mathrm{ee}}^{-1}(\varepsilon, T)
$$

where $\tau_{\mathrm{ee}}^{-1}$ is given by Eq. (97). Substitution of the modified DOS into Eqs. (66)- 68) yields an additional factor $\exp \left(-T^{2} / T_{\mathrm{q}}^{2}\right)$ in the amplitude of MIRO for $T$ above the temperature $T_{\mathrm{q}}$ defined by $2 \pi / \omega_{\mathrm{c}} \tau_{\text {ee }}\left(0, T_{\mathrm{q}}\right)=1$ [which gives $T_{\mathrm{q}} \sim\left(\omega_{\mathrm{c}} \varepsilon_{F}\right)^{1 / 2}$ up to a logarithmic factor], see Figs. 8 and 13 b,c. It is worth recalling that electronelectron scattering produces no additional exponential damping for the $\mathrm{SdH}$ oscillations, see footnote 6. The difference in the manifestation of electron-electron interactions in the damping factor for $\mathrm{SdH}$ oscillations and in the damping factor for MIRO is related to the fact that the former oscillations emerge at linear order in $\delta$, while the latter-at order $\delta^{2}$.

g. Relative weight of the inelastic and displacement contributions. The discussion in Secs. III.B.1.d-f shows that the relative weight of $\sigma^{\text {dis }}$ and $\sigma^{\text {in }}$ in the amplitude of MIRO strongly depends on $T$ and the correlation properties of disorder. For an estimate of their relative importance, we omit the anisotropic part [Eq. (77)] and the subleading for $\omega \gg \omega_{c}$ term proportional to $\mathcal{R}_{3}[\mathrm{Eq}$. (84) $]$ in $\sigma^{\text {dis }}$, and combine Eqs. (77)-(79) and (82)-(84) into

$$
\frac{\sigma^{\mathrm{in}}+\sigma^{\mathrm{dis}}}{\sigma_{\mathrm{D}}} \sim-\left(\frac{2 \tau_{\mathrm{in}}}{\tau}+\frac{\tau}{2 \tau_{\star}}\right) 4 \delta^{2} \mathcal{P} \frac{\pi \omega}{\omega_{\mathrm{c}}} \sin \frac{2 \pi \omega}{\omega_{\mathrm{c}}} .
$$

Since $\tau_{\text {in }} \sim \varepsilon_{F} / T^{2}$ (Sec. III.B.1.e), Eq. (99) defines the temperature $T_{\star} \sim\left(\varepsilon_{F} \tau_{\star}\right)^{1 / 2} / \tau$ at which $\sigma^{\text {dis }}=\sigma^{\text {in }}$. In the case of smooth disorder [when $\tau_{\star}^{-1}$ is given by the second term in Eq. (94)], Eq. (99) yields

$$
\frac{\sigma^{\text {in }}}{\sigma^{\text {dis }}}=\frac{4 \tau_{\text {in }}(T) \tau_{\star}}{\tau^{2}} \simeq \frac{\tau_{\text {in }}(T)}{3 \tau_{\mathrm{q}}}, \quad \frac{\tau}{\tau_{\text {sh }}}<\frac{4 \tau_{\mathrm{q}}}{\tau} .
$$

For overlapping LLs, Eq. (100) gives $T_{\star} \sim\left(\varepsilon_{F} / \tau_{\mathrm{q}}\right)^{1 / 2} \gg$ $T_{\mathrm{q}} \sim\left(\omega_{\mathrm{c}} \varepsilon_{F}\right)^{1 / 2}$, i.e., in the case of smooth disorder, the inelastic contribution dominates in the whole temperature range $T \lesssim T_{\mathrm{q}}$ where $\mathrm{MIRO}$ can be observed
(Fig. 13b,c). By contrast, for the case of a strong shortrange component of disorder $\left(\tau_{\mathrm{sh}} \sim \tau \sim \tau_{\star}\right)$, one has $T_{\star} \sim T_{\mathrm{q}}\left(\tau_{\star} / \omega_{\mathrm{c}} \tau^{2}\right)^{1 / 2}$, much smaller than $T_{\mathrm{q}}$ for $\omega_{\mathrm{c}} \tau \gg 1$. As a result, the $\sigma^{\text {in }}$-dominated $T^{-2}$ dependence of the MIRO amplitude for $T<T_{\star}$ crosses over as $T$ is increased to a plateau (whose height is given by $\sigma^{\text {dis }}$ ) in the interval $T_{\star}<T<T_{\mathrm{q}}$ (Fig. 13b) before starting to fall off exponentially for $T>T_{\mathrm{q}}$. In the intermediate case of $T_{\star} \sim T_{\mathrm{q}}$, the range of $T$ in which MIRO are $T$ independent shrinks to zero (Fig. 13.).

The experimental results, presented in Fig.8, are in overall agreement with the above theory. Apart from the $T$ dependence, the two mechanisms are different in the photoresponse at the "odd nodes" $\omega / \omega_{\mathrm{c}}=n+1 / 2$, where $\sigma^{\text {in }}=0$ while $\sigma^{(\text {dis })} \propto \mathcal{R}_{3} \neq 0$, see Eqs. (77),$(79)$, and (84). Moreover, their polarization dependence is different (Sec.III.B.1.b). Experimental studies of these features [omitted in the estimate (99)] can serve as an additional tool to quantify the relative magnitude of $\sigma^{\text {dis }}$ and $\sigma^{\text {in }}$.

\section{Saturation of the inelastic contribution at high radiation power and/or in a strong dc field}

When the inelastic contribution dominates the oscillatory photoresponse (Sec.III.B.1.g), the leading correction to $\sigma^{\text {in }}$ linear in $\mathcal{P}$ and independent of $E$ [Eq. (82)] is given by the terms of higher order in $\mathcal{P}$ and $E^{2}$ in the distribution function. These are governed by the kinetic equation (68), a generalized - to include higher powers of $\mathcal{P}$ and $E^{2}$-solution of which in the regime of overlapping LLs reads (Dmitriev et al., 2005)

$$
\begin{aligned}
& \delta f=\delta \frac{\omega_{c}}{2 \pi} \frac{\partial f_{\varepsilon}^{T}}{\partial \varepsilon} \sin \frac{2 \pi \varepsilon}{\omega_{c}} \mathcal{F}\left(\tau_{\text {in }}, \mathcal{P}, \zeta\right) \\
& \mathcal{F}\left(\tau_{\text {in }}, \mathcal{P}, \zeta\right)=\frac{\mathcal{P} \frac{2 \pi \omega}{\omega_{c}} \sin \frac{2 \pi \omega}{\omega_{c}}+2 \zeta^{2}}{\tau / \tau_{\text {in }}+\mathcal{P} \sin ^{2} \frac{\pi \omega}{\omega_{c}}+\zeta^{2} / 2}
\end{aligned}
$$

where the dimensionless parameter

$$
\zeta \equiv \pi \epsilon_{\mathrm{dc}}=2 \pi e E R_{c} / \omega_{\mathrm{c}}
$$

characterizes the strength of the dc field. The current induced by the inelastic mechanism is then given by

$$
j_{d}=\sigma_{\mathrm{D}} E\left[1+2 \delta^{2}-2 \delta^{2} \mathcal{F}\left(\tau_{\text {in }}, \mathcal{P}, \zeta\right)\right] .
$$

The dependence of the resulting magnetoresistivity on $\mathcal{P}$ and $E$ is illustrated in Fig. 14.

Let us consider first the limit $E \rightarrow 0$. At fixed $\omega / \omega_{\mathrm{c}}$, MIRO saturate with increasing microwave power:

$$
\mathcal{F}\left(\tau_{\text {in }}, \mathcal{P}, 0\right) \rightarrow \frac{4 \pi \omega}{\omega_{c}} \cot \frac{\pi \omega}{\omega_{c}}, \quad \mathcal{P} \sin ^{2} \frac{\pi \omega}{\omega_{c}} \gg \frac{\tau}{\tau_{\text {in }}}
$$

In the limit of high power $\mathcal{P} \gg \tau / \tau_{\text {in }}$, the maxima and minima of MIRO at $\epsilon_{\mathrm{ac}}=\epsilon_{\mathrm{ac}}^{ \pm}[\mathrm{Eq}$. (63)] shift towards the nearest nodes at $\epsilon_{\mathrm{ac}}=n$, with the amplitude of MIRO 
at the extrema being proportional to $\left(\tau_{\text {in }} \mathcal{P} / \tau\right)^{1 / 2}$ :

$$
\epsilon_{\mathrm{ac}}^{ \pm}=n \mp \frac{1}{\pi}\left(\frac{\tau}{\tau_{\mathrm{in}} \mathcal{P}}\right)^{1 / 2},\left.\quad \mathcal{F}\right|_{\epsilon_{\mathrm{ac}}=\epsilon_{\mathrm{ac}}^{ \pm}}=\mp 2 \pi\left(\frac{\tau_{\mathrm{in}} \mathcal{P}}{\tau}\right)^{1 / 2}
$$

Note that the $T$ dependence changes in the nonlinear regime. As follows from Eq. (102), there remains - for arbitrarily strong $\mathcal{P}$ - a range of $\epsilon_{\text {ac }}$ around the nodes within which the photoresponse is linear in $\mathcal{P}$ (apart from heating by microwaves which can modify $\tau_{\text {in }}$ ); however, this range shrinks with increasing $\mathcal{P}$. In a broader vicinity of $\omega / \omega_{c}=n$ which includes the nearest maxima and minima of MIRO, the result (104) appliesunder the conditions $\tau_{\mathrm{q}} \ll \omega_{r}^{-1} \ll \tau \lesssim \tau_{\text {in }}$, which are met in typical experiments (Dmitriev et al., 2007a, 2005; Hatke et al., 2011a; Khodas and Vavilov, 2008)-for arbitrary microwave power and for arbitrary relation between the components of mixed disorder (93). The resulting behavior with $\mathcal{P}$ reproduces the observations in Fig.77, and is also in agreement with other experimental results (Mani et al., 2010; Ye et al., 2001).

For $\tau_{\mathrm{sh}} \sim \tau$, i.e., in the presence of a strong shortrange component of disorder, one should for $\tau_{\text {in }} \lesssim \tau$ take into account the displacement contribution and also multiphoton processes (the latter are important at $\mathcal{P} \sin ^{2} \pi \omega / \omega_{\mathrm{c}} \gtrsim 1$, see Sec.VI.B.4). This results in Eq. (106) with a factor of order unity substituted for $\tau_{\text {in }} / \tau$ (Hatke et al., 2011a; Khodas and Vavilov, 2008). If only the smooth component of disorder is present, Eqs. (104), (102) are applicable and give the main contribution to $j_{d}$ in a broader range $\mathcal{P} \sin ^{2} \pi \omega / \omega_{\mathrm{c}}<\tau / \tau_{\mathrm{q}}$; at higher $\mathcal{P}$, both the excitation of higher angular and temporal harmonics of the distribution function and the multiphoton processes become important, which results in the emergence of a series of distinct strongly nonequilibrium regimes as $\omega / \omega_{\mathrm{c}}$ is varied [for more details see (Dmitriev et al., 2007a)]. The higher harmonics of the distribution function also determine the Hall photoresponse (Sec.III.B.4).

The above consideration of the nonlinear-in- $\mathcal{P}$ effects [Eqs. (102), (106)] shows that the linear-response conductivity [Eq. (104) at $E \rightarrow 0$ ] can become negative despite $\delta \ll 1$, see Fig. 14, As discussed in Sec.IV.B this causes an electric instability leading to the formation of domains. The electric field $E^{*}$ in the domains can be obtained from the current-voltage characteristics (104), as illustrated in the inset to Fig. 14, see Sec.IV.C for details. The behavior of the magnetooscillations at high microwave power and in a strong dc field is further discussed in Secs. V, VI.

\section{General approach: Quantum kinetic equation}

A comprehensive approach to nonequilibrium transport in high LLs was initially formulated for the case of smooth disorder (Dmitriev et al., 2007a, 2005; Vavilov and Aleiner, 2004) and later generalized to the

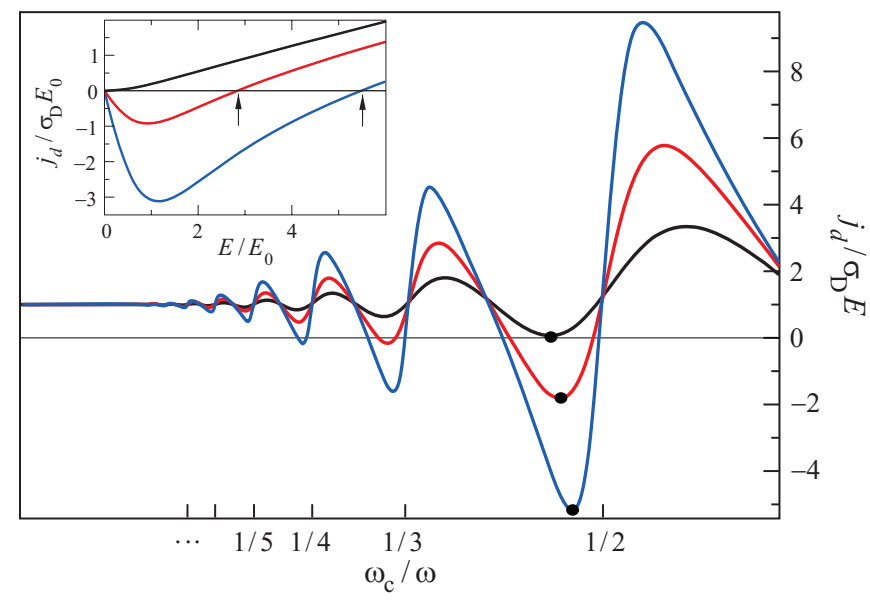

FIG. 14 Conductivity normalized to the dark Drude value (given, as we assume $\omega_{\mathrm{c}} \tau \gg 1$, by $j_{d} / \sigma_{\mathrm{D}} E$ ) as a function of $\omega_{c} / \omega$ at fixed $\omega \tau_{\mathrm{q}}=2 \pi$ for different levels of microwave power $\left.\left(\tau_{\text {in }} / \tau\right) \mathcal{P}\right|_{\omega_{\mathrm{c}}=0}=0.24$ (bottom), 0.8, 2.4 (top). Inset: $I-V$ characteristics at the minima (marked by the dots) of the $\omega / \omega_{c}$ dependence of the resistivity. The arrows show the value of the dc field $E^{*}$ in domains in units of $E_{0}=\pi \omega_{\mathrm{c}} \tau / 2 \tau_{\text {in }} e R_{c}$. Adapted from Dmitriev et al. (2005).

case of generic [Eq. (71)] disorder (Dmitriev et al., 2009b; Khodas and Vavilov, 2008; Vavilov et al., 2007). The key steps of the derivation are highlighted as follows.

(1) Transformation $\mathbf{r} \rightarrow \mathbf{r}-\boldsymbol{\xi}(t)$ to a moving coordinate frame, where $\boldsymbol{\xi}(t)$ obeys

$$
\partial_{t} \boldsymbol{\xi}(t)=\left(\frac{\partial_{t}-\omega_{c} \hat{\varepsilon}}{\partial_{t}^{2}+\omega_{c}^{2}}\right) \frac{e}{m}\left[\boldsymbol{E}_{\mathrm{dc}}+\boldsymbol{E}_{\omega}(t)\right]
$$

with $\hat{\varepsilon}_{x y}=-\hat{\varepsilon}_{y x}=1$, is similar to the transformation to Floquet states, used in a number of works (Auerbach and Pai, 2007; Kashuba, 2006b; Lee and Leinaas, 2004; Lvapilin and Patrakov, 2006; Park, 2004; Torres and Kunold, 2005; Volkov and Takhtamirov, 2007) on the displacement mechanism of MIRO. This transformation (unambiguously defined for $\omega \neq \omega_{c}$ ) eliminates homogeneous electric fields in the Hamiltonian of a clean electron system at the expense that the impurity potential, "dressed by the electric fields," becomes time-dependent. Scattering off the moving impurities can then change the energy of electrons.

(2) Keldysh equations within the SCBA. In the moving frame, the Green's functions $\hat{G}^{\alpha}$ and the self-energies $\hat{\Sigma}^{\alpha}(\alpha=R, A, K$ refer to the retarded, advanced, and Keldysh components) are related within the SCBA (Sec. II.C.1) as

$$
\hat{\Sigma}_{21}^{\alpha}=\int \frac{d^{2} q}{(2 \pi)^{2}} W_{q} e^{-i \boldsymbol{q} \boldsymbol{\xi}_{21}}\left(e^{i \boldsymbol{q} \hat{\mathbf{r}}} \hat{G}^{\alpha} e^{-i \boldsymbol{q} \hat{\mathbf{r}}}\right)_{21},
$$

where $W_{q}$ is the correlation function of the bare disorder potential, the subscript (21) denotes times $t_{2}$ and $t_{1}$ on the Keldysh contour, and $\boldsymbol{\xi}_{21}=\boldsymbol{\xi}\left(t_{2}\right)-\boldsymbol{\xi}\left(t_{1}\right)$. 
(3) Quasiclassical approximation. For a degenerate 2DEG in high LLs, Eq. (108) can be reduced to a simpler quasiclassical equation in the "action-angle" representation:

$$
\begin{aligned}
& \Sigma_{21}^{\alpha}(\varphi)=-i \hat{\mathcal{K}}_{21} g_{21}^{\alpha}(\varphi), \\
& g_{21}^{\alpha}(\varphi) \equiv i \omega_{\mathrm{c}} \sum_{k} \hat{G}_{21}^{\alpha}(\hat{n}+k ; \hat{\varphi}),
\end{aligned}
$$

where the operators $\hat{n}$ and $\hat{\varphi}$ are canonically conjugated, $[\hat{n}, \hat{\varphi}]=-i$. The eigenvalues of $\hat{n}$ and $\hat{\varphi}$ are the LL index and the angle coordinate of the momentum, respectively. The effects of disorder and external fields are encoded in the integral operator $\hat{\mathcal{K}}$,

$$
\hat{\mathcal{K}}_{21} F(\varphi)=\int \frac{d \varphi^{\prime}}{2 \pi} \frac{e^{i k_{\mathrm{F}}\left(\boldsymbol{n}_{\varphi}-\boldsymbol{n}_{\varphi^{\prime}}\right) \boldsymbol{\xi}_{21}}}{\tau_{\varphi-\varphi^{\prime}}} F\left(\varphi^{\prime}\right)
$$

The distribution function $\hat{f}$, defined by $\hat{G}^{R}-\hat{G}^{A}-\hat{G}^{K}=$ $2\left(\hat{G}^{R} \hat{f}-\hat{f} \hat{G}^{A}\right)$, commutes with $\hat{\varphi}$. Accordingly, the operator $\hat{\varphi}$, which enters Eq. (109) and the impurity collision integral,

$$
i \operatorname{St}_{i m}\{f\}=\hat{\Sigma}^{R} \hat{f}-\hat{f} \hat{\Sigma}^{A}+\left(\hat{\Sigma}^{K}+\hat{\Sigma}^{A}-\hat{\Sigma}^{R}\right) / 2,
$$

can be treated as a $c$-number. The result is the quantum kinetic equation

$$
\begin{aligned}
\left(\partial_{t}+\omega_{\mathrm{c}} \partial_{\varphi}\right) f_{21} & -\mathrm{St}_{\mathrm{in}}\{f\}_{21}=\mathrm{St}_{\mathrm{im}}\{f\}_{21} \\
\operatorname{St}_{\mathrm{im}}\{f\}_{21} & =\int d t_{3}\left[\hat{\mathcal{K}}_{21}\left(g_{23}^{R} f_{31}-f_{23} g_{31}^{A}\right)\right. \\
& \left.-f_{31} \hat{\mathcal{K}}_{23} g_{23}^{R}+f_{23} \hat{\mathcal{K}}_{31} g_{31}^{A}\right] .
\end{aligned}
$$

Here $t=\left(t_{1}+t_{2}\right) / 2$ is the "center-of-mass" time. The inelastic collision integral $\mathrm{St}_{\text {in }}\{f\}_{21}$ accounts for electronelectron scattering and for the coupling to a thermal (phonon) bath.

Except for specific cases (Sec.VI.A.3) in the regime of separated LLs, the effect of external fields on the spectrum can be neglected, meaning $g_{t_{1}-t_{2}}^{R}$ in the moving frame remains the same as in the static frame and does not depend on $t$ and $\phi$. It follows that the direct current

$$
\boldsymbol{j}=2 e v_{F} \int d \varepsilon \nu(\varepsilon)\left\langle\overline{\boldsymbol{n}_{\varphi} f(\varepsilon, \varphi, t)}\right\rangle-2 e \nu_{0} \varepsilon_{F} \overline{\partial_{t} \boldsymbol{\xi}(t)}
$$

is determined by the first angular harmonic of the Wigner-transformed distribution function $f(\varepsilon, \varphi, t)$. The bar denotes time averaging over the period of the microwave field in the steady state.

The approach formulated above [Eqs. (111), (113)(115)] validates the description of the inelastic and displacement mechanisms of MIRO in Sec.III.B.1 It has been applied to a wealth of nonequilibrium phenomena that we address in the remaining part of the review.
4. Quadrupole and photovoltaic contributions to the photoconductivity

Careful study of the quantum kinetic equation (113) shows (Dmitriev et al., 2007a) that there are in total four different contributions to the photocurrent at the leading order $E E_{\omega}^{2}$. Diagrams (A)-(D) in Fig. 15 represent four ways to obtain perturbatively the first angular harmonic $f_{10}$ of the distribution function $f(\varepsilon, \varphi, t) \equiv$ $\sum f_{\nu n} e^{i \nu \varphi+i n \omega t}$, which defines the current (115), starting from the isotropic dark distribution $f_{\varepsilon}^{T}$. In the displacement contribution $(\mathrm{A}), f_{10} \propto\left(\omega_{\mathrm{c}} \partial_{\varphi}\right)^{-1} \mathrm{St}_{\mathrm{im}}\left\{f_{\varepsilon}^{T}\right\} \mathrm{re}-$ sults directly from the action of the collision operator $\mathrm{St}_{\mathrm{im}} \propto E E_{\omega}^{2}$. In the inelastic contribution (B), $f_{10} \propto$ $\left(\omega_{\mathrm{c}} \partial_{\varphi}\right)^{-1} \mathrm{St}_{\mathrm{im}}\left\{\delta f_{00}\right\}$, where $\mathrm{St}_{\mathrm{im}} \propto E$ and $\delta f_{00} \propto \tau_{\mathrm{in}} E_{\omega}^{2}$ is the microwave-induced correction to the isotropic part of $f$. Apart from $\delta f_{00}$, the action of $\mathrm{St}_{\mathrm{im}} \propto E_{\omega}^{2}$ on $f_{\varepsilon}^{T}$ results in excitation of the second angular harmonics $f_{20}$. The linear dc response in the resulting state, $f_{10} \propto\left(\omega_{\mathrm{c}} \partial_{\varphi}\right)^{-1} \mathrm{St}_{\mathrm{im}}\left\{f_{20}\right\}$ with $\mathrm{St}_{\mathrm{im}} \propto E$, produces the "quadrupole" contribution $(\mathrm{C})$ to the direct current. Finally, in the "photovoltaic" mechanism (D), a combined action of the microwave and dc fields $\mathrm{St}_{\mathrm{im}} \propto$ $E E_{\omega}$ produces nonzero temporal harmonics $f_{10}$ and $f_{12}$. The ac response in the resulting state, $f_{10} \propto\left(\partial_{t}+\right.$ $\left.\omega_{\mathrm{c}} \partial_{\varphi}\right)^{-1} \mathrm{St}_{\mathrm{im}}\left\{f_{10}, f_{12}\right\}$ with $\mathrm{St}_{\mathrm{im}} \propto E_{\omega}$, also gives rise to the direct current.

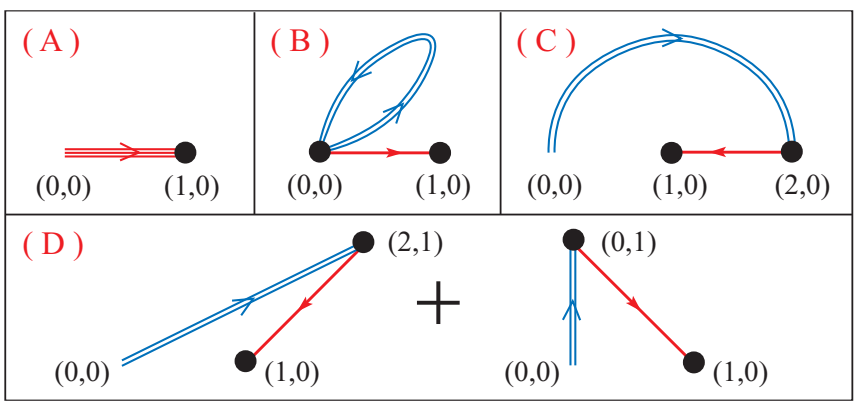

FIG. 15 Diagrams representing four distinct quantum contributions (A)-(D) to the photocurrent at order $E E_{\omega}^{2}$. Adapted from Dmitriev et al. (2007a).

Calculations performed in (Dmitriev et al., 2007a) (for smooth disorder) and (Dmitriev et al., 2009b) (for generic disorder) show that all four mechanisms (A)-(D) produce microwave-induced corrections to the direct current $\mathbf{j}-\mathbf{j}_{\text {dark }}=\hat{\sigma}_{p h} \boldsymbol{E}$. The full photoconductivity tensor $\hat{\sigma}^{(\mathrm{ph})} \propto E_{\omega}^{2}$ consists of components of different symmetry

$$
\hat{\sigma}^{(\mathrm{ph})}=-2 \delta^{2} \sigma_{\mathrm{D}}\left(\begin{array}{ll}
d_{s}+d_{a} & h_{s}+h_{a} \\
h_{s}-h_{a} & d_{s}-d_{a}
\end{array}\right)
$$

oscillating with $\omega / \omega_{\mathrm{c}}$. The Hall part is solely due to mechanisms (C) and (D). In particular, the quadrupole mechanism (C) yields an unusual symmetric off-diagonal term $h_{s}$ violating the Onsager symmetry, while the antisymmetric $h_{a}$ is produced by the photovoltaic mechanism (D). The photovoltaic mechanism also yields corrections 
to both isotropic $\left(d_{s}\right)$ and anisotropic $\left(d_{a}\right)$ diagonal components. It is worth mentioning that, unlike contributions (A)-(C), the photovoltaic contribution (D) cannot be described in terms of the scattering rates similar to (66). In particular, it involves not only the DOS given by the imaginary part of $G^{R}$ but also quantum corrections to the real part of the Green's function which do not appear in the golden-rule approximation.

In the case of smooth disorder, the largest contribution comes from the inelastic mechanism (B), which contributes only to $d_{s}$. In this case, $d_{s} \gg d_{a} \sim h_{s} \sim h_{a}$, meaning the contributions of subleading mechanisms (A), (C), and (D) are comparable in magnitude. A sufficiently large amount of short-range scatterers enhances contribution (A), see Sec.III.B.1.d while the magnitude of contributions (C) and (D) remains essentially the same, $d_{s} \sim d_{a} \gg h_{s} \sim h_{a}$. Thus, the description of MIRO in Sec.III.B.1, which accounts only for the inelastic and displacement contributions, is justified as long as the diagonal part of $\hat{\sigma}^{(\mathrm{ph})}$ is concerned. Mechanisms (C) and (D) yield oscillations in the Hall component (in the terms $h_{a}$ and $h_{s}$ ). Upon tensor inversion, the terms $d_{a}$ and $d_{a}$ also contribute to the Hall components of the resistivity tensor. Although these contributions are suppressed by an additional small factor $1 / \omega_{c} \tau$, they may compete with those originating from $h_{s, a}$, since $d_{s, a}$ may be much larger than $h_{s, a}$. Microwave-induced oscillations in the Hall resistivity were observed experimentally (Mani et al., 2004a; Studenikin et al., 2004; Wiedmann et al., 2011b). While the amplitude of the oscillations in the Hall component observed by Wiedmann et al. (2011b) is in good agreement with theory, Mani et al. (2004a); Studenikin et al. (2004) reported considerably stronger oscillations. In fact, a similar issue is known for $\mathrm{SdH}$ oscillations whose measured amplitude in the Hall component of the resistivity often considerably exceeds the theoretical predictions. It is likely that the physical mechanism of the enhancement of the Hall component is the same in both cases.

\section{Classical mechanisms of MIRO}

Most experimental results on MIRO reported so far have been consistently explained within the quantum kinetic approach described above. These quantum effects are directly linked (Dmitriev et al., 2003; Fedorych et al., 2010) to quantum magnetooscillations $\sigma^{(\mathrm{q})}(\omega)$ in the dynamic conductivity (Sec.II.C.3). On the other hand, the quasiclassical memory effects, discussed in Sec.II.B.3. also produce large $\omega / \omega_{\mathrm{c}}-$ oscillations in the ac conductivity, $\sigma^{(c)}(\omega)$ [Eqs. (23), (24)], see the comparison of the classical and quantum contributions in Fig. 2, In turn, the oscillatory $\sigma^{(\mathrm{c})}(\omega)$ translates into $\omega / \omega_{\mathrm{c}}$-oscillations of the electronic temperature $T_{e}$, while the oscillating $T_{e}$ manifests itself in dc transport via the renormalization of the elastic scattering rate by electron-electron interac- tions. The resulting oscillatory contribution to the photoconductivity has the form (Dmitriev et al., 2004)

$$
\frac{\Delta \sigma_{\mathrm{ph}}^{(c)}(0)}{\sigma_{\mathrm{D}}} \sim-\frac{\tau_{\text {in }}^{\mathrm{e}-\mathrm{ph}}}{\tau} \mathcal{P} \frac{\omega^{2}}{\varepsilon_{F} T} \frac{\Delta \sigma^{(c)}(\omega)}{\sigma_{\mathrm{D}}(\omega)},
$$

where $\tau_{\text {in }}^{\mathrm{e}-\mathrm{ph}}$ is the electron-phonon inelastic relaxation time (which controls the heating of the 2DEG) and $\Delta \sigma^{(c)}$ is given by Eqs. (23) and (24). The phase of the magnetooscillations in $\Delta \sigma_{\mathrm{ph}}^{(c)}(0)$ is opposite to the phase in the dynamical conductivity $\Delta \sigma^{(c)}(\omega)$ and is shifted by $\pi / 4$ with respect to the quantum oscillations [Eqs. (82) and (77)]. At low $T$, the magnitude of the oscillations in Eq. (117) may become comparable to that in Eq. (82) since the ratio $\Delta \sigma^{(c)}(\omega) / \sigma_{\mathrm{D}}(\omega)$ can be of the order of unity (Fig. 2), while the small parameter $\omega / \varepsilon_{F} \sim 10^{-1}-10^{-2}$ is easily compensated by the large ratio $\tau_{\text {in }}^{\mathrm{e}-\mathrm{ph}} / \tau_{\text {in }}$ of the electron-phonon and electronelectron inelastic relaxation times [electron-electron scattering provides for relaxation of the fast oscillations in the energy distribution of electrons in Eq. (82) but conserves the total energy of the electron gas]. However, unlike the quantum oscillations of the photoconductivity, the oscillations described by Eq. (117) saturate with increasing $\mathcal{P}$ well before $\Delta \sigma_{\mathrm{ph}}^{(c)}(0) / \sigma_{\mathrm{D}}$ becomes of order unity, and therefore, cannot cause ZRS. Namely, in the regime of strong heating $\left(T_{e}-T \gtrsim T\right), \Delta \sigma_{\mathrm{ph}}^{(c)}(0) / \sigma_{\mathrm{D}}$ does not exceed $T_{e} / \varepsilon_{F} \ll 1$.

It is important to mention that all classical corrections to the Drude formula induced by the homogeneous external ac and dc fields vanish to zero in the Boltzmann equation framework in the case of a parabolic dispersion relation and $\varepsilon$-independent $\tau$ (Dmitriev et al., 2004). Apart from the interaction-induced effects discussed above, finite nonlinear corrections may result either from the energy dependence of $\tau$ [which typically occurs on the scale of $\varepsilon_{F}$, thus yielding an additional small factor $T / \varepsilon_{F}$ compared to Eq. (117) (Dmitriev et al., 2004)] or from a weak nonparabolicity [which leads to the appearance of an even larger energy scale of the order of the bandgap in the denominator (Joas et al., 2004; Koulakov and Raikh, 2003)]. By contrast, the Landau quantization provides the much smaller scale $\omega_{\mathrm{c}}$ in the dependence on $\varepsilon$ [Eqs. (78) and (83)], which explains the leading role of the quantum mechanisms of MIRO.

Two recent works addressed classical effects close to the boundary of a 2DEG. Chepelianskii and Shepelyansky (2009) studied the influence of the microwave field on the phase space portrait of electron dynamics near the sample edge. The conclusion was that at $\omega / \omega_{\mathrm{c}}=n+1 / 4$ the microwave radiation tends to trap electrons in trajectories propagating near the edge, while at $\omega / \omega_{\mathrm{c}}=n$ the particles are more efficiently kicked out into the bulk. Mikhailov (2011) discussed an effective electrostatic (ponderomotive) potential for electrons (whose cyclotron dynamics is modeled using Newton's equation 
with a friction term), created by a strong inhomogeneity (Mikhailov and Savostianova, 2006) of the microwave field in the near-contact region. Depending on the ratio $\omega / \omega_{\mathrm{c}}$, the ponderomotive force repels electrons from or attracts them to the near-contact area. Since the above works did not develop a systematic theoretical analysis of the contributions of the boundary effects to the resistance of the $2 \mathrm{D}$ system, it is difficult to compare these contributions to those predicted by the bulk theory (Sec.III.B.1). In any case, in the systems in which MIRO are observed transport is dominated by the bulk contribution, so that even a strong modification of edge transport may modify the total current only weakly. Indeed, experimentally, no dependence of the MIRO amplitude on the sample dimension or geometry, characteristic of the edge effects, has been reported so far. Moreover, edge transport obviously plays no role in Corbino geometry. Furthermore, recent observations of MIRO using various contactless techniques (Andreev et al. ., 2011; Bykov et al., 2010b) have ruled out the contact-related phenomena as a generic cause of MIRO.

\section{Related microwave-induced phenomena}

\section{Microwave-induced photovoltaic effects}

Recent experiments Bykov, 2008a; Dorozhkin et al., 2009), performed using an asymmetric contact configuration, discovered that microwaves can induce oscillatory current and voltage signals very similar to MIRO even in the absence of external dc driving, see Fig. 16. These photovoltaic effects were attributed (Dmitriev et al., 2009a) to the violation of the Einstein relation between the conductivity and the diffusion coefficient, induced by the microwave illumination. Consider an inhomogeneous system characterized by the electrostatic potential $\phi(\mathbf{r})$ and the electron density $n_{e}(\mathbf{r})$, both smoothly varying in space. In equilibrium, the electron system is characterized by the constant electrochemical potential $\eta=e \phi(r)+n_{e}(\mathbf{r}) / \chi^{(\text {dark })}$, while a generic weak perturbation leads to the current $j^{\text {(dark) }}=-\sigma^{(\text {dark })} \nabla \phi-$ $e D^{\text {(dark) }} \nabla n_{e} \equiv-e D^{\text {(dark) }} \chi^{(\text {dark })} \nabla \eta$. At $2 \pi^{2} T / \omega_{\mathrm{c}} \gg 1$, the dark compressibility $\chi^{\text {(dark) }}=2 \nu_{0}$, so that the Einstein relation in equilibrium reads $\sigma^{\text {(dark) }}=2 e^{2} \nu_{0} D^{\text {(dark) }}$, where $D^{\text {(dark) }}=\left\langle\tilde{\nu}^{2}(\varepsilon)\right\rangle_{\varepsilon} R_{c}^{2} / 2 \tau$, see Eq. (76)).

Analysis of Eqs. (65)- (68), adapted to the inhomogeneous conditions, shows (Dmitriev et al., 2009a) that the direct current under microwave illumination,

$$
j=-\sigma_{\eta} \nabla \phi-2 e \nu_{0} D \nabla \eta,
$$

necessarily contains an extra "anomalous term" $-\sigma_{\eta} \nabla \phi$ which violates the Einstein law. The term $\sigma_{\eta}=\sigma^{\text {in }}+\sigma_{1}^{\text {dis }}$ includes the inelastic contribution to MIRO (82) and the most important part $\sigma_{1}^{\text {dis }} \propto \mathcal{R}_{1}$ of the displacement contribution (77). The other part $\sigma_{3}^{\text {dis }} \propto \mathcal{R}_{3}$ enters the nonequilibrium diffusion coefficient, $D=D^{\text {(dark) }}+$

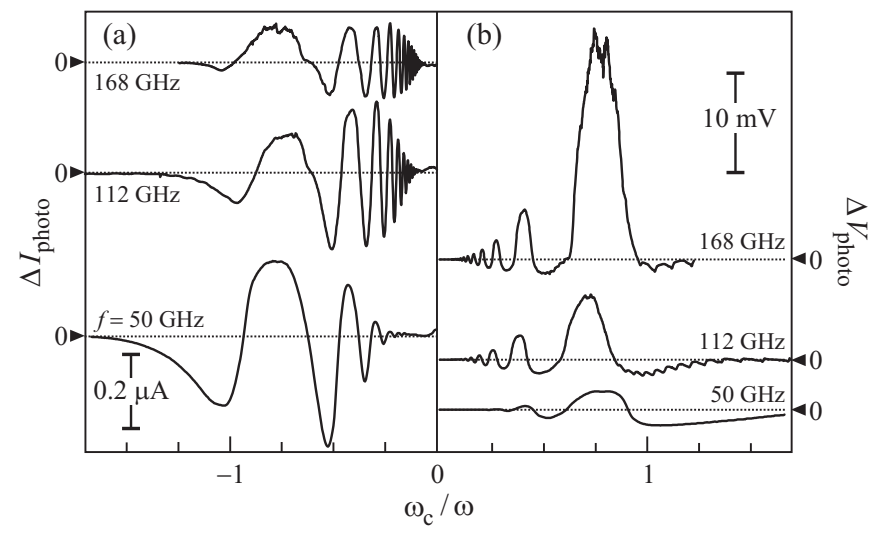

FIG. 16 (a) Photovoltaic current and (b) voltage at a fixed radiation frequency $f=50,112$, and $168 \mathrm{GHz}$ versus $\omega_{c} / \omega$ in the absence of dc bias applied to the sample. The curves are offset for clarity. Adapted from Dorozhkin et al. (2009).

$\sigma_{3}^{\text {dis }} / 2 e^{2} \nu_{0}$. The total conductivity $\sigma$, which defines the direct current $j=\sigma E$ in a homogeneous system, is given by $\sigma=\sigma_{\eta}+2 e^{2} \nu_{0} D$.

The current (118) is nonzero even at $\nabla \eta=0$, provided there is a nonzero built-in electric field (created, e.g. by an asymmetric contact configuration or by local gates), as observed in (Bvkov, 2008a, 2010; Dorozhkin et al., 2009, 2011b; Willett et al., 2004). The simplest model, proposed by Dmitriev et al. (2009a), assumes that the builtin field $\mathcal{U}_{c} / L$ is provided by two contacts with the difference of work functions $\mathcal{U}_{c}$, attached to a 2DEG stripe of width $L$. The $I-V$ characteristic for this setup reads

$$
j=\sigma_{\eta} \mathcal{U}_{c} / L+\sigma V / L
$$

where the voltage between the contacts $\left.\mathrm{eV} \equiv \eta\right|_{x=0}-$ $\left.\eta\right|_{x=L}$. As seen from Eq. (119), there is a finite oscillatory photocurrent $j$ at zero bias voltage $V=0$, as well as a finite photovoltage $V$ (asymmetric with respect to $V=0$ ) at $j=0$, as shown in Fig.16. The corresponding field and density distributions are calculated in (Dorozhkin et al., 2011a).

\section{Microwave-induced compressibility oscillations}

The violation of the Einstein relation under microwave illumination has a number of other important consequences. In particular, the generalized compressibility $\chi_{q}^{(\phi)} \equiv n_{q} /\left(-e \phi_{q}\right)$, defined as a static density response $n_{q} e^{i q r}$ to a weak local perturbation $\phi_{q} e^{i q r}$ of the screened electrostatic potential, strongly deviates from its equilibrium value $\chi^{\text {(dark) }}=2 \nu_{0}$. Assuming a stationary nonequilibrium state with the homogeneous component of the current $j=0$, one obtains from Eq. (118):

$$
\chi_{q \rightarrow 0}^{(\phi)}=2 \nu_{0}+\sigma_{\eta} / e^{2} D=\sigma / e^{2} D .
$$

Note that, in contrast, the quantity $\chi_{q}^{(\mu)} \equiv n_{q} / \mu_{q}$ is not modified: $\chi_{q}^{(\mu)}=2 \nu_{0}$. Using the generalized compress- 
ibility, one can define the nonequlibrium screening length (Dorozhkin et al., 2011a)

$$
\lambda=\frac{\epsilon D}{2 \pi \sigma} \equiv \frac{\epsilon}{2 \pi e^{2} \chi_{q}^{(\phi)}},
$$

which replaces the equilibrium Thomas-Fermi screening length $\lambda_{0}=\epsilon / 2 \pi e^{2} \chi^{\text {(dark) }}$ in all electrostatic problems.

At $q^{-1} \gg l_{\text {in }} \equiv\left(D \tau_{\text {in }}\right)^{1 / 2}$ but $q R_{c} \ll 1$, the local approximation for the current (118) is still justified. At the same time, the effect of spatial variations of the electric field on the distribution function becomes essential (Vavilov et al., 2004). Summarizing the results of Dmitriev et al. (2009a) and Vavilov et al. (2004) for the leading inelastic contribution, the generalized compressibility at $q R_{c} \ll 1$ is given by

$$
\frac{\chi_{q}^{(\phi)}}{2 \nu_{0}}=1-\delta^{2} \mathcal{F}\left(\tau_{\text {in }}, \mathcal{P}, \zeta\right) \frac{2+q^{2} l_{\text {in }}^{2}}{1+q^{2} l_{\text {in }}^{2}},
$$

meaning the amplitude of the oscillations in $\chi_{q}^{(\phi)}$ reduces by a factor of 2 at $q l_{\text {in }} \gg 1$ compared to the limit $q \rightarrow 0$ [function $\mathcal{F}$ is defined in (102)]. These results suggest that ZRS corresponds to a plateau in the compressibility, as illustrated in Fig. 17] for the case $q l_{\text {in }} \gg 1$. Therefore, local measurements of the compressibility [e.g., using the techniques that utilize single-electron transistors (Ilani et al., 2000, 2001)] may provide a real space snapshot of the domain structure in ZRS. Experimental work in this direction is currently underway.

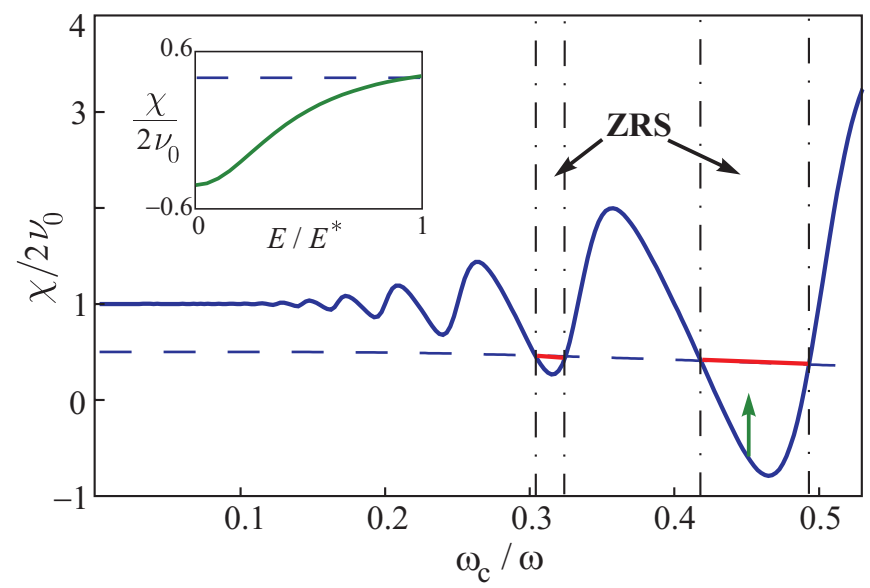

FIG. 17 Microwave-induced oscillations of the compressibility as a function of $\omega_{\mathrm{c}} / \omega$ at fixed $\omega \tau_{\mathrm{q}}=2 \pi$ and $\left.\left(\tau_{\text {in }} / \tau\right) \mathcal{P}\right|_{\omega_{\mathrm{c}}=0}=$ 1. In ZRS, the electric field inside domains $E^{*}$ fixes the compressibility at the level shown by a dashed line. Inset: the dependence of the compressibility on the electric field $E$ inside the domain wall. Adapted from Vavilov et al. (2004).

\section{Effects of a parallel magnetic field}

Yang et al. (2006) observed the effect of a parallel magnetic field $B_{\|}$on MIRO: the oscillations were suppressed as $B_{\|}$is increased and virtually disappeared at
$B_{\|} \sim 0.5 \mathrm{~T}$. This observation came as a surprise because, according to the theory presented above, MIRO is an orbital effect and should not be essentially sensitive to the spin degree of freedom. Moreover, the transverse motion of $2 \mathrm{D}$ electrons (that would be affected by the parallel field) is frozen. In (Mani, 2005), MIRO were indeed practically independent of $B_{\|}$up to about $1 \mathrm{~T}$, i.e., in the same range of $B_{\|}$as in Yang et al. (2006). The most likely explanation of the experimental data of Yang et al. (2006) is the effect of $B_{\|}$on the quantum scattering rate $1 / \tau_{\mathrm{q}}$. Indeed, the parallel magnetic field does affect the structure of the wave function across the 2DEG plane, which can in general modify the impurity scattering rate. This is a nonuniversal effect, as it depends on microscopic details of the nanostructure in which the 2DEG is formed, as well as on the character of imperfections. It is plausible that in the QW used by Yang et al. (2006) this effect may be stronger than in a typical single-interface structure. In fact, a closer inspection of the experimental data of Yang et al. (2006) shows that the dark resistivity (and thus the transport scattering rate $1 / \tau$ ) was enhanced by the parallel field by a factor of about 3 . While in general the enhancement factors for $1 / \tau$ and $1 / \tau_{\mathrm{q}}$ may be different (because $1 / \tau$ and $1 / \tau_{\mathrm{q}}$ may be controlled by different components of disorder), this clearly shows that $B_{\|}$did strongly enhance disorder-induced scattering. The effects of $B_{\|}$were also studied by Hatke et al. (2011f) in the nonlinear (with respect to the dc field) transport regime, see Sec.V.A It was demonstrated that $B_{\|}$suppresses quantum oscillations via the enhancement of $1 / \tau_{\mathrm{q}}$.

\section{MIRO in multisubband structures}

The microwave-induced effects in magnetotransport have also been intensively studied in structures with several occupied subbands, both experimentally (Bykov et al., 2008, 2010d; Gusev et al., 2011; Wiedmann et al., 2010b.c, 2011b, 2008, 2009b) and theoretically (Raichev, 2008; Wiedmann et al., 2010b, 2008, $2009 \mathrm{~b})$. Already the first experiments in a double QW in the Hall-bar geometry (Wiedmann et al., 2008) as well as in van der Pauw and Corbino geometries (Bvkov et al., 2008) demonstrated strong interference between MIRO and MISO (the latter are discussed in Sec. II.C.4). Specifically, it was observed that (i) MIRO are strongly enhanced (suppressed) in the maxima (minima) of MISO and that (ii) MISO in the minima of MIRO are inverted by sufficiently strong microwave radiation. Similar effects were recently observed also in triple QWs (Wiedmann et al., 2009b).

Wiedmann et al. (2008) performed a direct comparison of the experimental results with theory that combines the inelastic mechanism of MIRO (Dmitriev et al., 2005) and the intersubband scattering effects (Raichev, 2008). The theory reproduced with a good accuracy the complicated interference pattern, as well as the nontriv- 
ial dependence of the combined oscillations on the microwave power, frequency, and temperature, observed in the experiment. In all cases (Wiedmann et al., 2010b, 2008, 2009b), the comparison confirmed the $T^{-2}$ dependence of MIRO amplitude (Dmitriev et al., 2005) up to $T \sim 4 \mathrm{~K}$. Similar $T^{-2}$ dependence of the inelastic scattering time was extracted from the exponential high- $T$ damping of MISO (Mamani et al., 2008). This damping, governed by the interaction-induced broadening of LLs, has the same origin as the high- $T$ damping of MIRO discussed in Sec.III.B.1.e.

In the case of a balanced double QW, with negligible interlayer correlations of the scattering potential and identical scattering rates, the theoretical result of Raichev (2008) reads [cf. Eq. (104) and Eqs. (58)-(60)]

$$
\frac{j_{d}}{\sigma_{\mathrm{D}} E}-1=\delta^{2}\left[1-\mathcal{F}\left(\tau_{\mathrm{in}}, \mathcal{P}, \zeta\right)\right]\left(1+\cos \frac{2 \pi \Delta}{\omega_{\mathrm{c}}}\right)
$$

where $\mathcal{F}$ is given by Eq. (102). This expression clearly shows features (i) and (ii) mentioned above. Indeed, MISO are inverted for $\mathcal{F}>1$. Further, if $2 \delta^{2}(\mathcal{F}-1)$ exceeds unity, the conductivity (and, therefore, resistivity) at the MISO maximum becomes negative, which leads to the emergence of ZRS (Sec. IV]). ZRS of this kind have been observed by Wiedmann et al. (2010c), see Fig. 18 .

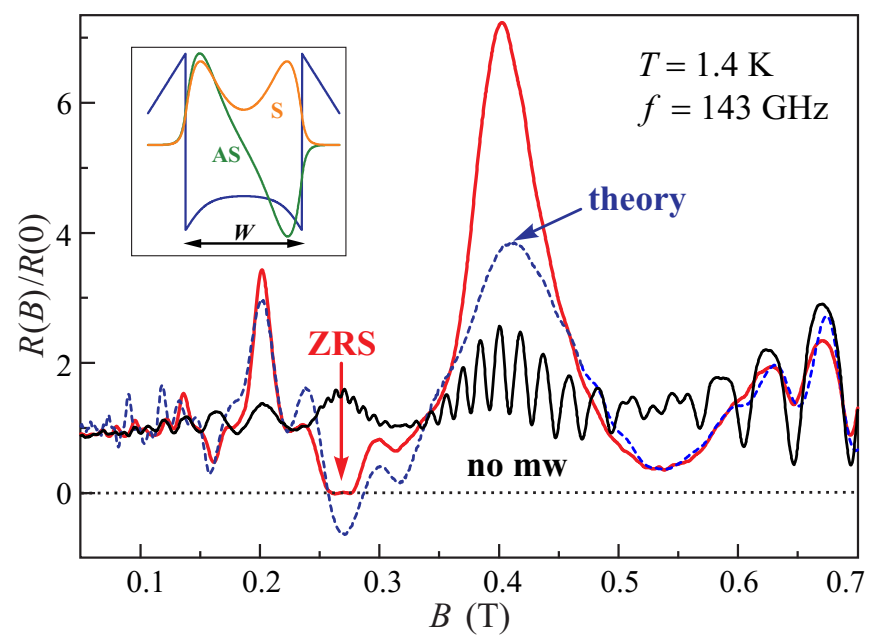

FIG. 18 Measured resistance (solid lines) of a two-subband electron system without (no mw) and under microwave irradiation at $f=143 \mathrm{GHz}$ as a function of $B$. The inverted by microwaves MISO peak at $B \simeq 0.27 \mathrm{~T}$ exhibits vanishing resistance. Dashed line: theory. Inset: symmetric (S) and antisymmetric (AS) wavefunctions for the two lowest subbands in a wide QW. From Wiedmann et al. (2010c).

\section{MIRO in spatially modulated systems}

The discussion in Secs. III.C.1 and III.C.2 shows that the violation of the Einstein relation strongly modifies the transport and thermodynamic properties of the microwave-illuminated 2DEG already in the presence of a weak smooth inhomogeneity. The opposite limit of a strong short-period spatial modulation was studied in (Dietel, 2006; Dietel et al., 2005; Joas et al., 2005; Kennett et al., 2005; Robinson et al., 2004; Torres and Kunold, 2006).

Dietel (2006); Dietel et al. (2005); Joas et al. (2005) examined the transport properties of the 2DEG with a unidirectional static modulation [this system has been intensively studied before in the context of Weiss oscillations and transport anisotropies (Beenakker, 1989; Gerhardts et al., 1989; Mirlin and Wölfle, 1998; Weiss et al., 1989; Zhang and Gerhardts, 1990)]. In the presence of a periodic potential $V(x)=\tilde{V} \cos Q x$ the degeneracy of LLs is lifted and LL bands appear with the dispersion relation

$$
\varepsilon_{n k}=\omega_{\mathrm{c}}(n+1 / 2)+\tilde{V} J_{0}\left(Q R_{c}\right) \cos \left(Q k l_{\mathrm{B}}^{2}\right),
$$

where the Bessel function $J_{0}$ is an approximation that is valid for high LLs. The DOS of the clean modulated system has square-root singularities at the band edges. In the $x$ direction, the photoconductivity can be calculated using the golden-rule approach formulated in Sec. III.B.1. The results for the displacement and inelastic mechanisms are given by Eqs. (77) and (82) with the oscillating factors (78), (79), and (83) calculated using the DOS corresponding to Eq. (124). The oscillating factors acquire additional sign changes, related to singular DOS, which can be detected in the experiment. In the perpendicular direction, the linear (in the dc field) photoresponse diverges for the displacement mechanism. The divergence is cut off by inelastic processes. As a result, both the displacement and inelastic contributions to the transverse photoresponse are proportional to $\tau_{\text {in }}$ and have a similar magnitude.

Kennett et al. (2005); Robinson et al. (2004) studied the magnetoresistivity in the presence of surface acoustic waves (SAW). This perturbation combines (Levinson et al., 1998) both the unidirectional spatial modulation with the wavevector $Q$ and the ac excitation at frequency $\omega=s Q$, where $s$ is the sound velocity. Apart from an ac analog of the Weiss oscillations, which results in an anisotropic positive MR oscillating with both $Q R_{c}$ and $\omega / \omega_{\mathrm{c}}$, Kennett et al. (2005); Robinson et al. (2004) calculated the associated quantum contribution for the inelastic mechanism of MIRO, which potentially gives rise to SAW-induced ZRS. The quantum contribution is given by Eqs. (104) and (102), where the microwave power $\mathcal{P}$ is replaced by properly normalized SAW power absorbed by the 2DEG. Since the absorbed SAW power is proportional to the dynamic conductivity of the classical system at the wavevector $Q$, $\sigma_{\omega Q}^{(c)}$, which oscillates with $Q R_{c}, \rho_{x x}$ (Fig. 19) shows additional $Q R_{c^{-}}$oscillations on top of relatively slow $\omega / \omega_{\mathrm{c}^{-}}$ oscillations identical to MIRO.

Yuan et al. (2006) investigated experimentally the microwave photoresistance in the presence of a triangular 


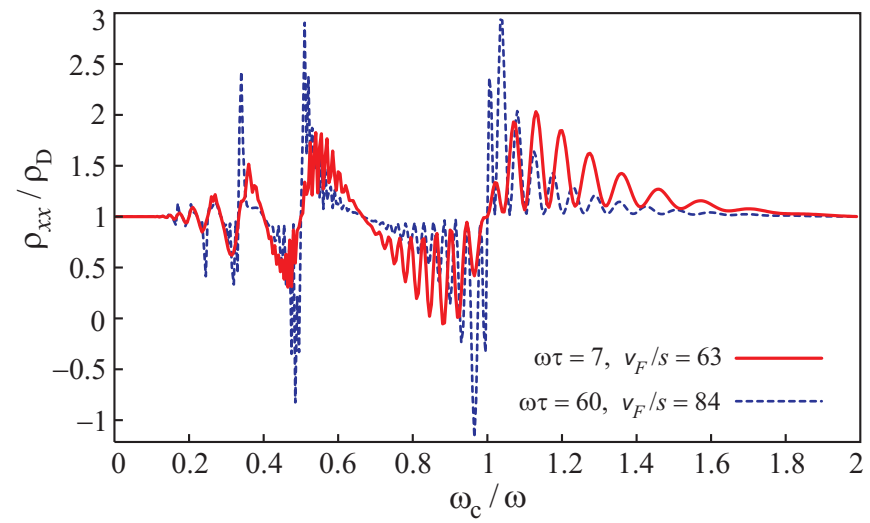

FIG. 19 Calculated resistivity magnetooscillations in the presence of a surface acoustic wave for $\omega \tau=7$ and $v_{F} / s=63$ (solid line) and $\omega \tau=60$ and $v_{F} / s=84$ (dashed), both curves for $\omega \tau_{\mathrm{q}}=6$ and $\omega \tau_{\text {in }}=302$. From Kennett et al. (2005).

antidot superlattice. This study revealed the conventional MIRO with a superimposed magnetoplasmon peak on top of a series of narrow geometrical resonances positioned at $2 R_{c}=\gamma_{n} a$, where $a$ is the lattice period and $\gamma_{n}$ are numbers of order unity corresponding to various commensurate orbits. Essentially no interference between MIRO and the commensurability oscillations was observed. Note, however, that a strong interplay of the two types of oscillations was predicted (Torres and Kunold, 2006) for a short-period superlattice with $a$ of the order of $l_{\mathrm{B}} \ll R_{c}$.

\section{Microwave-induced $B$-periodic oscillations}

Kukushkin et al. (2004) discovered a different kind of photoresistance and photovoltage magnetooscillations in the region $\omega<\omega_{\mathrm{c}}$, see Fig.20. These $B$-periodic oscillations were attributed to the interference of edge magnetoplasmons (EMP) emitted from different potential probes along the Hall-bar edge. The condition for constructive interference of the EMP injected from two contacts separated by the distance $L$ reads $q L=\omega L / v=2 \pi N$, where the EMP velocity $v$ is proportional to the Hall conductivity $\sigma_{x y} \propto n_{s} / B$. This interpretation explains the period $\Delta B \propto n_{s} / \omega L$ found in the experiment. Kukushkin et al. (2004) established the following properties of the $B$ periodic oscillations: (i) the amplitude of the resistivity oscillations shows a threshold behavior as a function of $\mathcal{P}$, (ii) the photovoltage scales linearly with $\mathcal{P}$ in the limit of small $\mathcal{P}$ and saturates with increasing $\mathcal{P}$, (iii) the saturation power for the photovoltage is close to the threshold power for the resistivity oscillations, (iv) the resistivity and photovoltage oscillations are phase shifted by $\pi / 4$ with respect to each other, and (v) the threshold $\mathcal{P}$ for the resistivity oscillations is an order of magnitude lower for the microwave electric field perpendicular to the Hall-bar edge (which supports the EMP scenario). The phenomenon showed only a weak dependence on $T$ in the broad interval $T \sim 1-10 \mathrm{~K}$. The photovoltage reduced only by one order of magnitude at $T$ as high as $70 \mathrm{~K}$. Systematic theory of the $B$-periodic oscillations remains to be developed.

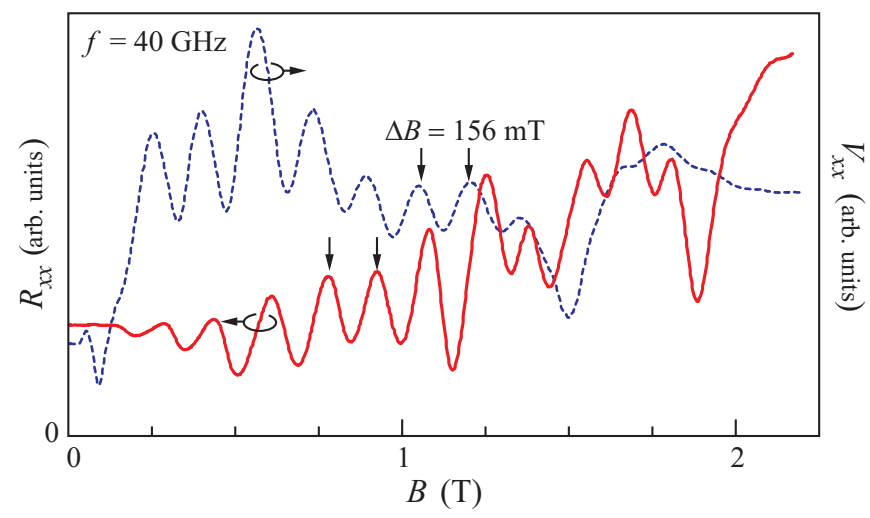

FIG. $20 B$-periodic magnetoresistance (solid line) and photovoltage (dashed) oscillations at $f=40 \mathrm{GHz}$ measured in a Hall bar with potential probes separated by $0.5 \mathrm{~mm}$. From Kukushkin et al. (2004).

Kukushkin et al. (2005) also reported a successful operation of the microwave spectrometer based on the $B$ periodic photovoltaic oscillations. The $B$-tunable selective detection was demonstrated in the frequency range $20 \mathrm{GHz}<f<150 \mathrm{GHz}$ and for temperatures up to $T \sim 80 \mathrm{~K}$ (with a foreseen possibility of extension to the $\mathrm{THz}$ frequency range). Implementation of plasmon resonances in such a device allows one to overcome the temperature limitation $T<\omega$ of the conventional selective detectors based on electronic transitions. The major advantage of magnetoplasmons is that they can be exploited at arbitrary $\omega \tau$ provided $\omega_{\mathrm{c}} \tau \gg 1$, while the operation of the conventional plasmonic $B=0$ detectors in field-effect transistors is limited to the region $\omega \tau>1$.

In a recent experiment Stone et al. (2007), where MIRO, ZRS, and $B$-periodic oscillations were observed simultaneously, the period $\Delta B$ of the $B$-periodic oscillations was reported to scale as $\Delta B \propto n_{s} / \omega$ and be independent of the distance $L$ between the contacts. This result is in contradiction to that by Kukushkin et al. (2004): the controversy has remained unresolved. Several other experiments, in particular, by Dorozhkin et al. (2007a); Yuan et al. (2006), reported no interference of the magnetoplasmon effects and MIRO with each other. This agrees with the calculation by Volkov and Takhtamirov (2007), according to which the magnetoplasmons can strongly affect MIRO only in the limit of well-separated LLs - which is out of the range explored in the above experiments. 


\section{RADIATION-INDUCED ZERO-RESISTANCE STATES (ZRS)}

\section{A. ZRS: Experimental discovery and basic properties}

Zero resistance is a rare occurrence in condensed matter physics, usually signaling a novel state of matter, such as superconductivity and QH effects (von Klitzing et al., 1980; Tsui et al., 1982), see Sec.IV.B.2 for further examples. Experiments by Mani et al. (2002) and by Zudov et al. (2003) on microwave-irradiated very-high mobility $\left(\mu \gtrsim 10^{7} \mathrm{~cm}^{2} / \mathrm{V} \cdot \mathrm{s}\right)$ 2DEG revealed that the lower order minima of MIRO (Sec.III.A) can extend all the way to zero forming the zero-resistance states (ZRS), see Fig.21. With appropriate microwave intensity, temperature, and sample quality, ZRS can span magnetic field ranges corresponding to several tens in filling factors. However, unlike the $\mathrm{QH}$ effect, vanishing of diagonal resistance in microwave-irradiated $2 \mathrm{DEG}$ is not accompanied by Hall quantization.

Over the past decade ZRS were observed over a wide frequency range, from as low as $9 \mathrm{GHz}$ (Willett et al., 2004) to as high as $254 \mathrm{GHz}$ (Smet et al., 2005). Experiments in ultra-high mobility $\left(\mu \gtrsim 2 \cdot 10^{7} \mathrm{~cm}^{2} / \mathrm{V} \cdot \mathrm{s}\right) 2 \mathrm{DEG}$ also revealed ZRS stemming from the minima of fractional MIRO (Sec.VI.A.1), namely near $\epsilon_{\mathrm{ac}}=3 / 2,1 / 2$ and 2/3 (Zudov et al., 2006b), as well as a ZRS presumably appearing due to a frequency mixing under bichromatic microwave irradiation (Zudov et al., 2006b). Recently, ZRS were also observed to emerge from the MISO maxima in double QWs (Wiedmann et al., 2010c), see Fig.18. Shortly after the discovery of ZRS, experiments by Yang et al. (2003) in Corbino-disk shaped 2DEG demonstrated the existence of corresponding zeroconductance states (ZCS) (Fig.22). Later studies by Bykov et al. (2010b) reported observation of ZCS in capacitively-coupled 2DEG. As discussed in Sec.VII.B.1. ZCS were also realized in a non-degenerate 2D electron system on liquid ${ }^{3}$ He surface (Konstantinov and Kono, 2010).

The temperature dependence of the ZRS has been examined by several groups (Mani et al., 2002; Willett et al., 2004; Zudov et al., 2003). All studies found that ZRS disappear with increasing temperature (see Fig.23), transforming into MIRO minima where the resistance approximately followed the Arrhenius law $\rho_{\omega} \propto \exp (-\Delta / T)$. The "energy gaps" $\Delta \propto B$ extracted in this way exceed relevant $T \sim \omega$ by an order of magnitude.

As discussed in Sec.IV.B a homogeneous state with negative resistivity is electrically unstable and is expected to break into domains with local current density at which the nonlinear resistance is zero (Andreev et al., 2003; Auerbach et al., 2005; Finkler and Halperin, 2009). To date direct confirmation of absolute negative resistivity and the domain model has been limited. First experimental support of the domain picture was provided by Willett et al. (2004) who observed large volt-
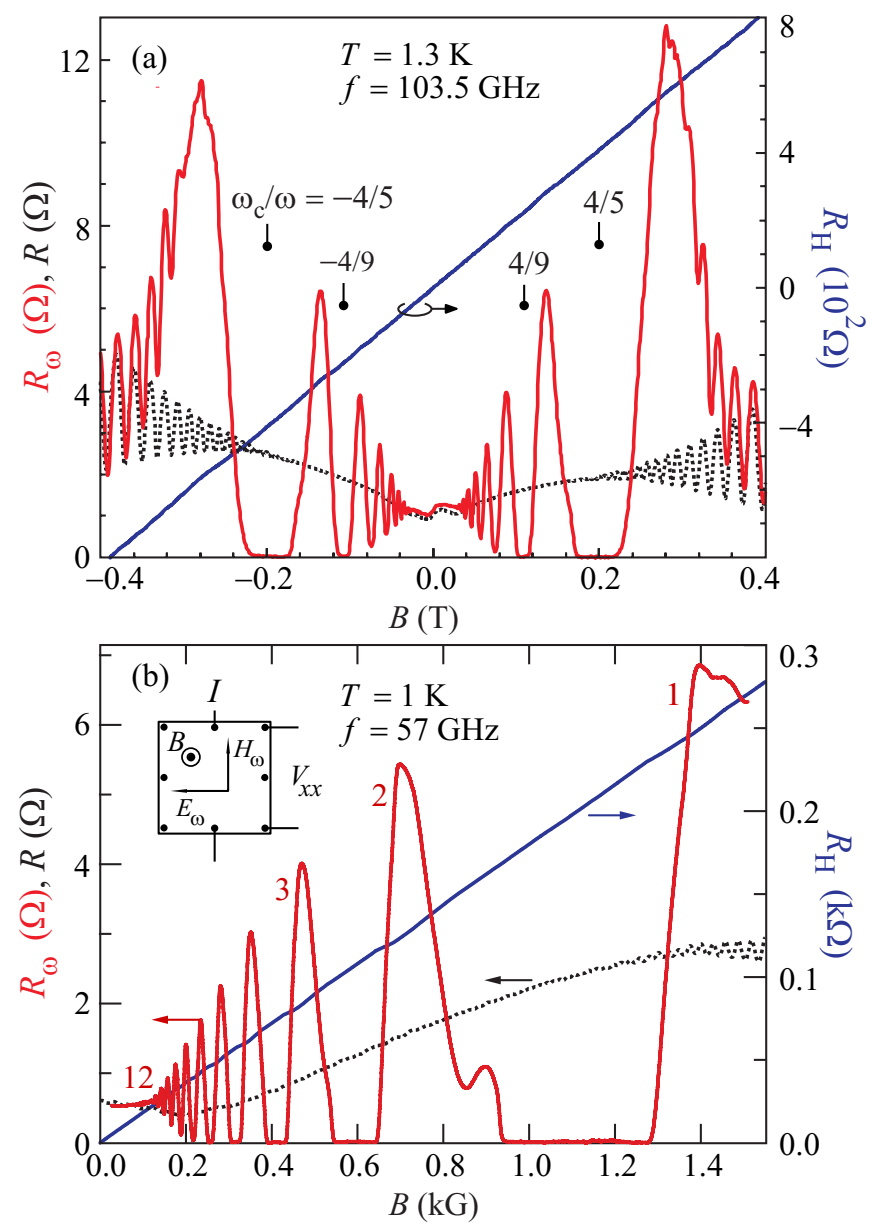

FIG. 21 Longitudinal $\left[R_{\omega}(B)\right.$, left axis $]$ and Hall $\left[R_{H}(B)\right.$, right axis] magnetoresistance under microwave irradiation. Longitudinal magnetoresistance $R(B)$ without irradiation is also shown. Parameters: (a) microwave frequency $f=103.5$ GHz, temperature $T=1.3 \mathrm{~K}$, electron density $n_{e} \simeq 3 \times 10^{11}$ $\mathrm{cm}^{-2}$, and mobility $\mu \simeq 1.5 \times 10^{7} \mathrm{~cm}^{2} / \mathrm{Vs}$; (b) $f=57 \mathrm{GHz}$, $T \simeq 1.0 \mathrm{~K}, n_{e} \simeq 3.5 \times 10^{11} \mathrm{~cm}^{-2}$, and $\mu \simeq 2.5 \times 10^{7} \mathrm{~cm}^{2} / \mathrm{Vs}$. Adapted from (a) Mani et al. (2002) and (b) Zudov et al. (2003).

ages between an internal and an external contact to 2DEG in the ZRS regime, see Sec.IV.C. Experiments by Zudov et al. (2006a) employing radiation of two distinct frequencies found that, away from ZRS, the bichromatic photoresistance can be approximated by a simple superposition of monochromatic photoresistances, e.g. $\delta \rho_{\omega_{1} \omega_{2}} \simeq \alpha_{1} \delta \rho_{\omega_{1}}+\alpha_{2} \delta \rho_{\omega_{2}}$. Theoretically the bichromatic photoresponse was addressed by Lei (2006); Lei and Liu (2006a). Assuming that such a superposition also holds in the regime where one of the frequencies gives rise to ZRS, e.g. underlying microscopic resistance is negative $\rho+\delta \rho_{\omega_{1}}<0$, but $\rho+\delta \rho_{\omega_{1} \omega_{2}}>0$, one can reconstruct negative resistance which is otherwise masked by instability (see Fig.24). In other words, the experiment uses one of the frequencies to probe the absolute negative resistance corresponding to a ZRS induced by another frequency.

Dorozhkin et al. (2011b) performed time-resolved 


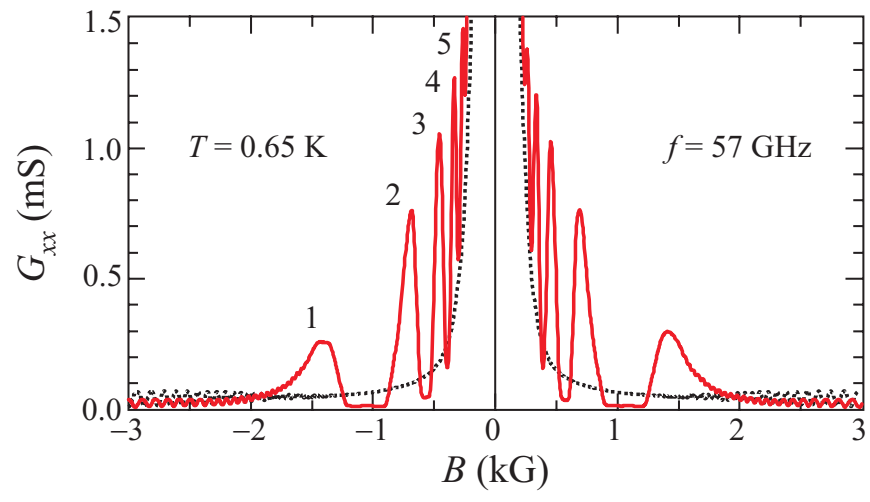

FIG. 22 Magnetoconductance $G_{x x}(B)$ under microwave irradiation of frequency $f=57 \mathrm{GHz}$. These data were obtained at $T=0.65 \mathrm{~K}$ on a Corbino-shaped 2DEG with inner (outer) diameter $0.5(3.0) \mathrm{mm}$, density $n_{e} \simeq 3.55 \times 10^{11} \mathrm{~cm}^{-2}$, and mobility $\mu \simeq 1.28 \times 10^{7} \mathrm{~cm}^{2} / \mathrm{Vs}$. Adapted from Yang et al. (2003).

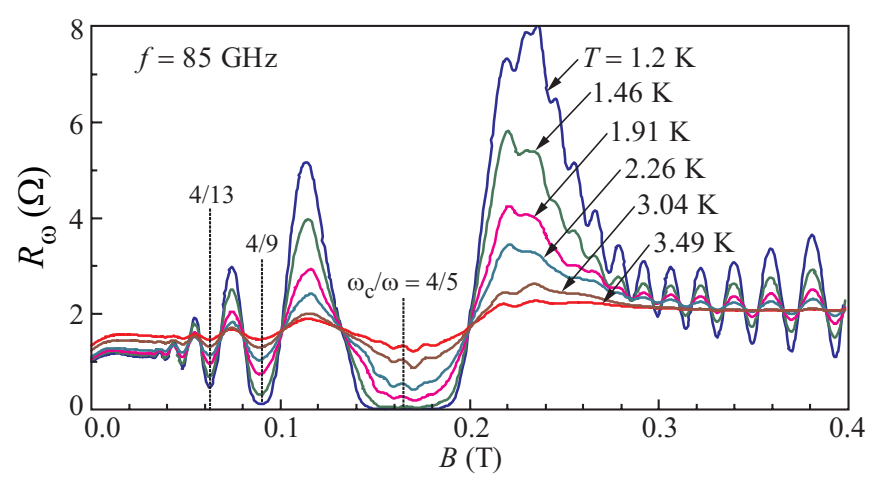

FIG. 23 Temperature evolution of ZRS and MIRO obtained under $f=85 \mathrm{GHz}$ illumination on the same sample as in Fig.21. Adapted from Mani et al. (2002).

measurements of Hall voltages between internal probes and observed random telegraph signals in the ZRS regime. These signals were interpreted in terms of spontaneous switching between two nearly degenerate configurations of spontaneously formed domains (Fig.25).

\section{B. Instabilities of a nonequilibrium 2D electron gas and the emergence of domains in a strong magnetic field: Phenomenological approach}

As discussed in Sec. III, for a sufficiently strong radiation power (and other parameters in the appropriate range), the linear resistivity of the microwave-illuminated 2DEG becomes negative. Such a state is unstable, which results in formation of spontaneous current and voltage breaking rotational symmetry (Andreev et al., 2003); the corresponding theory is presented in Sec. IV.B.1. In Sec. IV.B.2 we review some previously obtained results on related systems.

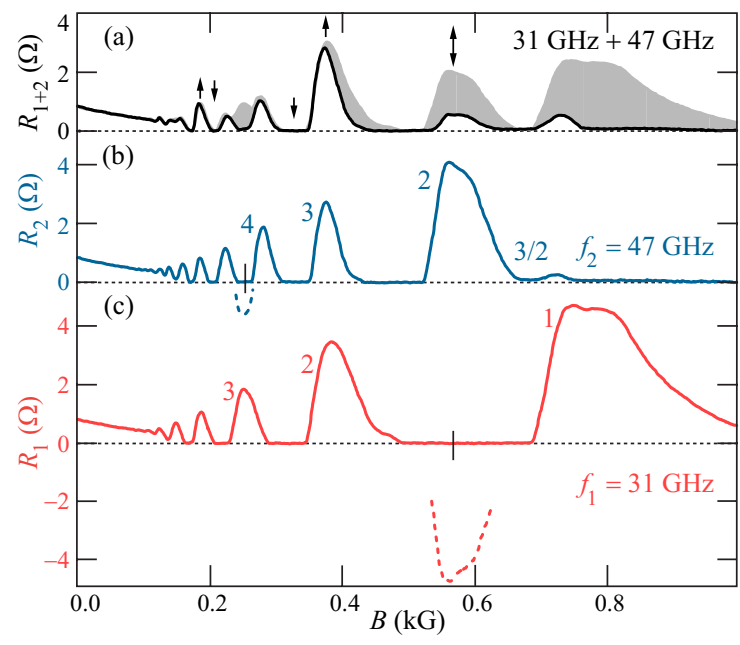

FIG. 24 Magnetoresistances (a) $R_{1}(B)$ at $f_{1}=31 \mathrm{GHz}$, (b) $R_{2}(B)$ at $f_{2}=47 \mathrm{GHz}$, and (c) $R_{1+2}$ at both $f_{1}$ and $f_{2}$. Maximum-maximum, minimum-minimum and maximumminimum overlaps are marked by $\uparrow, \downarrow$, and $\uparrow$, respectively. The upper boundary of the shaded area represents the average of monochromatic resistances $R_{1}$ and $R_{2}$. Dotted lines in (b) and (c) represent reconstructed negative resistance. These data were obtained on a 2 DEG $(\sim 5 \mathrm{~mm} \times 5 \mathrm{~mm})$ with density $n_{e} \simeq 3.6 \times 10^{11} \mathrm{~cm}^{-2}$ and mobility $\mu \simeq 2.0 \times 10^{7} \mathrm{~cm}^{2} / \mathrm{Vs}$. Adapted from Zudov et al. (2006a).

\section{ZRS effective theory: Spontaneous symmetry breaking}

The starting point of the theory of Andreev et al. (2003) is a model with a constant Hall resistivity $\rho_{H}$ and a nonlinear current dependence of the dissipative component $\mathbf{E}_{d}$ of the electric field:

$$
\mathbf{E}=\rho_{H} \mathbf{j} \times \hat{\mathbf{z}}+\frac{\mathbf{j}}{|\mathbf{j}|} E_{d}(|\mathbf{j}|) .
$$

It is assumed that the function $E_{d}(j)$ has a negative derivative at $j=0$ and a zero at $j=j_{c}$. The theory is phenomenological, i.e. it assumes that there is a certain microscopic mechanism that provides such currentvoltage characteristics. A distinct feature of the considered strong magnetic field regime is a duality between current and voltage. Specifically, Eq. (125) is appropriate in the Hall-bar geometry, when only one (say, $j_{x}$ ) component of the current is present; the two terms in Eq. (125) then give the Hall $\left(E_{y}\right)$ and the longitudinal $\left(E_{x}\right)$ field respectively. In contrast, for the Corbino-disc geometry one field component is zero (say, $E_{x}=0$ ) and one is interested in $\mathbf{j}\left(E_{y}\right)$. Since the Hall component is the dominant one in the conductivity tensor, the dependence of the field dependence of the dissipative component $j_{d}(E)$ is obtained from $E_{d}(j)$ in Eq. (125) by exchanging current and voltage (Bergeret et al., 2003),

$$
\mathbf{j}=\sigma_{H} \hat{\mathbf{z}} \times \mathbf{E}+\frac{\mathbf{E}}{|\mathbf{E}|} j_{d}(|\mathbf{E}|),
$$

with $\sigma_{H} \simeq\left(\rho_{H}\right)^{-1}$ and $j_{d}(E)=\sigma_{H} E_{d}\left(\sigma_{H} E\right)$. In other words, the dissipative current-voltage characteristics are 


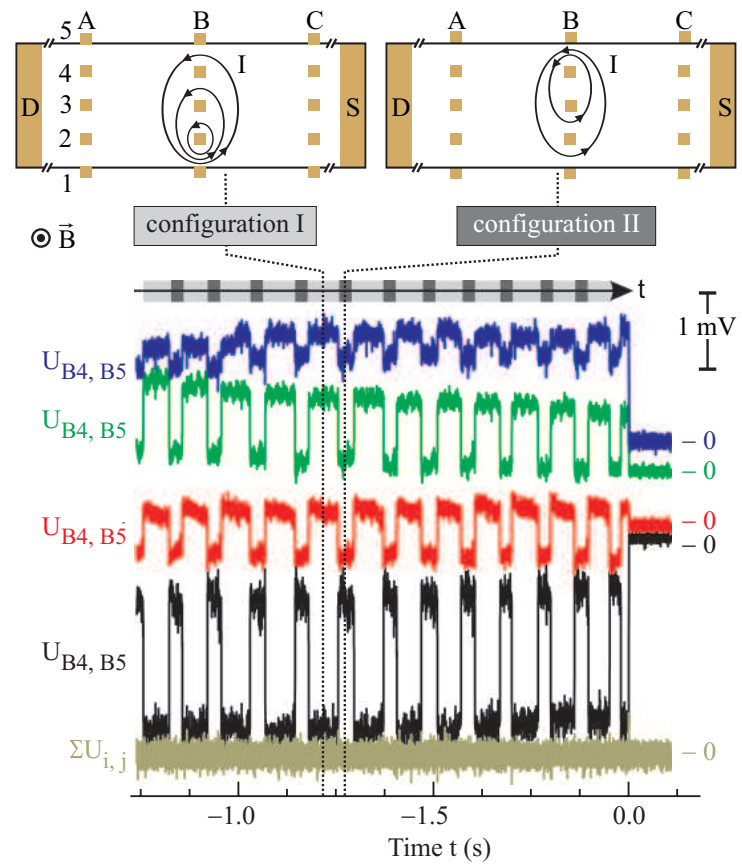

FIG. 25 Photovoltages (for $B=95 \mathrm{mT}, T=0.5 \mathrm{~K}$, and $f=48.1 \mathrm{GHz}$ ) across different pairs of adjacent contacts shown in the inset (middle column). The voltages at $t>0$ (no radiation) correspond to the zero reference level. Closed loops in the insets indicate the Hall current flow between the contacts in two different configurations. From Dorozhkin et al. (2011b).

S-shaped in the first case and N-shaped in the second case, see Fig. 26.
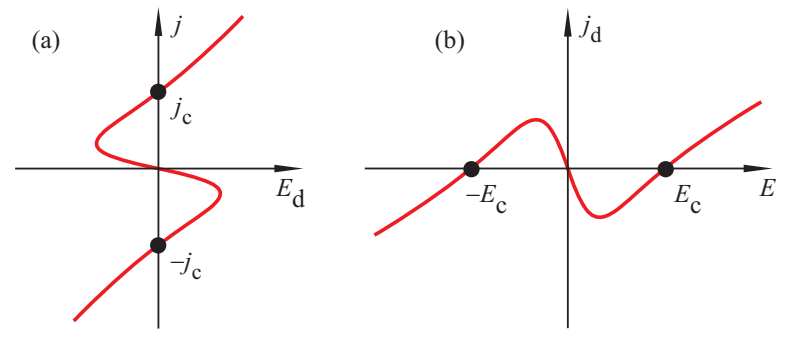

FIG. 26 S-shaped [(a), Hall bar setup, Eq. (125)] and Nshaped [(b), Corbino setup, Eq. (126)] current-voltage characteristics.

Equation (125) is supplemented by the continuity equation,

$$
\partial n_{e} / \partial t+\nabla \cdot \mathbf{j}=0
$$

Further,

$$
\mathbf{E}=-\nabla \phi(\mathbf{r}),
$$

where $\phi(\mathbf{r})$ is the electrostatic potential; its variations are related to those of density according to

$$
\delta \phi(\mathbf{r})=\int d^{2} r^{\prime} U\left(\mathbf{r}, \mathbf{r}^{\prime}\right) \delta n_{e}\left(\mathbf{r}^{\prime}\right)
$$

where $U\left(\mathbf{r}, \mathbf{r}^{\prime}\right)$ is the Coulomb interaction (that may be screened in the presence of an external gate). To explore the stability of the system, one considers a small deviation in density $\delta n_{e}(\mathbf{r}, t)$. Using Eqs. (126)-(129) and linearizing in $\delta n_{e}$, one gets

$$
\partial \delta n_{e} / \partial t=\boldsymbol{\nabla} \hat{\sigma}_{d} \boldsymbol{\nabla} \hat{U} \delta n_{e},
$$

where $\hat{\sigma}_{d}$ is the dissipative differential conductivity $\left(\sigma_{d}\right)_{\alpha \beta}=\partial\left(j_{d}\right)_{\alpha} / \partial E_{\beta}$ satisfying $\left(\sigma_{d}\right)_{\alpha \beta}=\left(\sigma_{d}\right)_{\beta \alpha}$. Note that the Hall component of the conductivity dropped out of Eq. (130) because of its antisymmetric character. The stability requires that the real part of all eigenvalues of the operator on the r.h.s. of Eq. (130) is nonpositive. Transforming it into the momentum space and using $U(q)>0$, the stability condition reduces to the requirement that eigenvalues of $\hat{\sigma}_{d}$ are positive, which yields

$$
\begin{aligned}
& d j_{d} / d E \geq 0 \\
& j_{d} / E \geq 0 .
\end{aligned}
$$

In other words, the stability requires that both absolute and differential conductivity are non-negative. As is clear from the above derivation, Eqs. (131) and (132) represent the stability to longitudinal and transverse fluctuations of the electric field with respect to its direction in the state under consideration.

Therefore, when the linear-response conductivity is negative the system is unstable near $j=E=0$ : fluctuations will grow until the stability point with current $j_{c}$ and electric field $E_{c}=\rho_{H} j_{c}$ is reached, see Fig.26. This implies that the system will break into domains with spontaneous currents and fields of these magnitudes. The simplest possible domain structure consists of two domains as shown in Fig. 27 for the case of Hall-bar and Corbino-disc setups (see Sec. IV.D for a discussion of more complicated domain structures). The values of spontaneous current and field correspond to dissipative current $j_{d}=0$ (i.e. zero absolute dissipative conductivity) in the Corbino disc setup and to dissipative field $E_{d}=0$ (and thus zero absolute dissipative resistivity) in the Hall bar geometry. When a finite current (for the Hall bar) or voltage (Corbino disc) is applied, the domain wall shifts to accommodate it. The system remains in the ZRS (or ZCS in the Corbino geometry) until the applied current (field) exceeds $j_{c}$ (respectively, $E_{c}$ ).

A further step forward in the study of the ZRS was done by Bergeret et al. (2003); Volkov and Pavlovskii (2004) who included on the r.h.s. of Eq. (126) the diffusive term $-e \hat{D} \nabla n_{e}$ and assumed that, contrary to the conductivity, the diffusion tensor is not affected by microwave radiation. TThis assumption was later justified by Dmitriev et al. (2009a).] This introduces in the problem the nonequilibrium screening length that governs the width of domain walls, see Sec.III.C.2. Volkov and Pavlovskii (2004) assumed however a 3D relation between the potential and the density [i.e. they assume $V(q) \sim 1 / q^{2}$ momentum dependence of the 


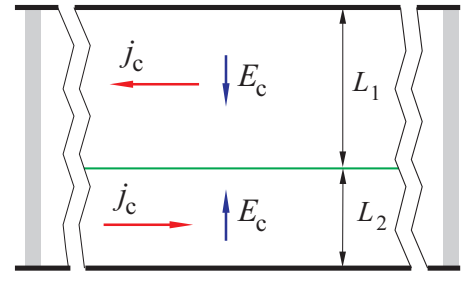

(a)

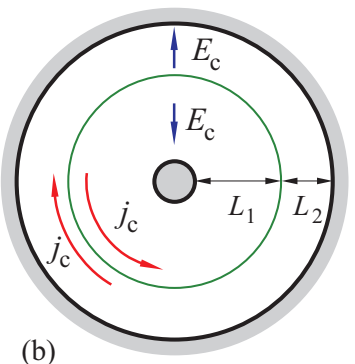

(b)
FIG. 27 Simplest domain structures for (a) Hall-bar and (b) Corbino-disc geometries. The current density $j_{c}$ and the electric field $E_{c}=\rho_{H} j_{c}$ in the domains are fixed by the condition (a) $E_{d}=0$ or (b) $j_{d}=0$, see Fig. 26 Corbino-disc [Hall-bar] system adapts to an applied bias $V=E_{c}\left(L_{1}-L_{2}\right)$ [applied current $I=j_{c}\left(L_{1}-L_{2}\right)$ ] remaining in ZCS [ZRS]. Adapted from Andreev et al. (2003); Vavilov and Aleiner (2004).

Coulomb interaction rather than $V(q) \sim 1 / q$ appropriate for 2D geometry, see (Dorozhkin et al., 2011a)] and, as a consequence, obtain the $3 \mathrm{D}$ screening length $\lambda_{3 D}=$ $\left(\epsilon D / 4 \pi\left|\sigma_{d}^{(3 D)}\right|\right)^{1 / 2}$. The resulting equations are used to find the spatial profile of the domain wall. The central result of Volkov and Pavlovskii (2004) is the residual resistivity of a finite sample in the ZRS phase (with a single domain wall assumed), which is found to be negative and exponentially small: $\rho_{\text {res }} \propto-\exp \left\{-L / \lambda_{3 \mathrm{D}}\right\}$. Here $L$ is the sample size in the direction transverse to the domain wall.

Dorozhkin et al. (2011a) established the electrical stability condition in a finite sample with realistic $2 \mathrm{D}$ Coulomb interaction. Instead of conditions (131) and (132) (which in the linear regime read $\sigma_{d}>0$ ), one gets $\sigma_{d}>-\epsilon D / 2 L$, or $\lambda^{-1}>\pi / L$ in terms of the $2 \mathrm{D}$ nonequilibrium screening length (121). In the diffusion-stabilized regime of negative conductivity, $-\epsilon D / 2 L<\sigma_{d}<0$, the theory of Dorozhkin et al. (2011a) predicts the emergence of two regions with opposite directions of electric field (this requires broken inversion symmetry, for instance, due to asymmetric contact configuration). The amplitude of these fields increases when the system approaches the instability threshold. This effect is a precursor of the domain structure in the regime $\sigma_{d}<-\epsilon D / 2 L$.

\section{Earlier results on related problems}

The instability of a semiconductor with dc negative conductivity was first pointed out by Zakharov (1960). Subsequently many performed detailed analyses of such instabilities in various systems with negative differential or absolute conductivity. The corresponding literature is extensive, and we restrict ourselves to quoting several early articles (Bonch-Bruevich and Kogan, 1965; Elesin and Manykin, 1967; Ridley, 1963; Volkov and Kogan, 1967), a review by Volkov and Kogan (1969), and the books by Bonch-Bruevich et al. (1975); Pozhela (1981); Schöll
(2001). The interested reader can find there an overview of various mechanisms of emergence of negative conductivity (characterized by N-shape or S-shape currentvoltage-characteristics), derivation of stability conditions, and an analysis of domain formation as a result of instabilities. In this context, particularly well known is the Gunn effect (Gunn, 1963): an N-type currentvoltage-characteristic leads to an instability resulting in the formation of moving domains and in microwave generation. The corresponding semiconductor device (Gunn diode) has found various application in high-frequency electronics.

The absolute negative conductivity was observed by Banis et al. (1972) [see also Sec. VI.3 in Pozhela (1981)]. This was achieved by exposing GaAs samples exhibiting a negative differential conductance to a microwave radiation. The explanation given by Banis et al. (1972); Pozhela (1981) assumes that the microwave field can be treated adiabatically and simply leads to an (oscillating in time) shift of the operation point of the device. After the time averaging of the corresponding differential conductivity this leads to a negative dc conductivity. It seems, however, that this explanation (that would be perfectly correct for a low-frequency field) does not fully catch the physics of the experiment. Indeed, the frequency of the microwave field (of order $10 \mathrm{GHz}$ ) does not seem to be small compared to inverse characteristic times in the device, so that the adiabaticity assumption is not met. Furthermore, the authors report that, in the absence of an external circuit, a large spontaneous dc voltage developed on the sample (whose polarity could be stabilized by a small symmetry breaking perturbation). The amplitude of the voltage was determined by the zero of the current-voltage characteristics, $I(V)=0$. This spontaneous formation of dc field domains resulting from the microwave-induced absolute negative conductivity indicates certain analogy between the experiment of Banis et al. (1972) and the systems constituting the subject of this review.

It was experimentally discovered (Basun et al., 1983; Liao et al., 1980) that intense laser illumination generates strong electric fields (detected via the splitting of luminescence lines) in ruby crystals. A phenomenological theory of this phenomenon was developed by Dyakonov (1984); Dvakonov and Furman (1984). It is based on an assumption that the absolute conductivity in ruby becomes negative for a sufficiently strong illumination power. The resulting instability leads to formation of electric field domains, thus explaining the experimental observations. There is a clear analogy between the theory of ZRS as resulting from regions of negative resistivity in MIRO and this theory of ruby domains. There are, however, also essential differences. First, is the presence of a strong magnetic field in the MIRO problem. Second, contrary to an isotropic $2 \mathrm{DEG}$, ruby is anisotropic: the electric field and the current are directed along the $C_{3}$ axis. This modifies the analysis of the stability (only longitudinal fluctuations need to be considered) and ren- 
ders the part of the current-voltage-characteristics with $I / V<0$ but $d I / d V>0$ stable. To our knowledge, no microscopic theory of negative conductivity in ruby has been developed.

The impact of a strong transverse magnetic field on this class of phenomena was also appreciated approximately half a century ago. Kazarinov and Skobov (1963) showed that a quantizing transverse magnetic field may lead under nonequilibrium conditions to strong nonlinearities in current-voltage-characteristics, in particular, to a negative differential resistance. The fact that in the case of large Hall angle $\left(\omega_{c} \tau \gg 1\right)$ current-voltagecharactertics have a dual shape in the Hall bar $\left(j_{y}=0\right)$ and Corbino disk $\left(E_{y}=0\right)$ geometries (in particular, an S-shaped characteristic of the Hall-bar device gives rise to an N-shaped characteristic for the Corbino disc) was pointed out by Bass (1965); Bogomolov et al. (1967). Kogan (1968) studied an instability in a system with negative differential resistance at intermediate Hall angles $\left(\omega_{c} \tau \sim 1\right)$. Elesin (1969); Gladun and Ryzhii (1970) and particularly Ryzhii (1970); Ryzhii et al. (1986) discussed the photoconductivity of a $2 \mathrm{D}$ gas in a quantizing magnetic field and strong electric field (see Sec.III.B) and concluded that it is possible to reach an absolute negative conductivity in this class of systems.

Another broad class of semiconductor systems in which similar phenomena have been intensively studied are single and multiple QW heterostructures. In particular, much work was devoted to resonant transmission via double-barrier structures, where a negative differential resistance was found (Sollner et al., 1983; Tsu and Esaki, 1973). More recently, it was predicted that under laser illumination the system may show absolute negative resistance (Dakhnovskii and Metiu, 1995). Further, the absolute negative conductivity was theoretically predicted (Ignatov et al., 1995; Pavlovich and Epshtein, 1976) and experimentally observed (Keay et al., 1995) in semiconductor superlattices under $\mathrm{THz}$ radiation; another theoretical work considers a superlattice in a magnetic field (Cannon et al., 2000). The theoretical predictions include generation of spontaneous voltages and currents resulting from instabilities. A negative absolute conductivity was also found in a related model Hartmann et al., 1997) of a particle in a periodic lattice driven by an ac field and coupled to a dissipative bath.

Absolute negative conductance under strongly nonequilibrium conditions emerges also in other types of systems. In particular, Aronov and Spivak (1975) predicted this effect to happen in a Josephson junction of two superconductors with different gaps one of which is subjected to light illumination. This prediction was experimentally confirmed by Gershenzon and Falei (1986, 1988). In view of the spatially local character of the junction, the negative conductance does not lead in this case to an instability (contrary to extended systems that tend to break into domains in such situations). Finally, an absolutely negative mobility was obtained in a purely classical model of a Brownian particle subjected to non- equilibrium noise (Eichhorn et al., 2002).

It is clear from the above discussion that many of the aspects of the ZRS problem have appeared earlier in related problems. On the other hand, to our knowledge, the combination of the key features of the ZRS problem, namely, (i) a strong magnetic field (large Hall angle), (ii) instability induced by an absolute negative dissipative conductivity, and (iii) 2D isotropy of the problem, has not appeared in any other context.

\section{Microscopic theory; determination of currents and fields in domains}

We combine now the phenomenological ZRS theory of Sec. IV.B.1 with microscopic calculations of photoresistivity. As discussed in Sec. III, the linear resistivity in the presence of microwaves may become negative around its minima. Specifically, consider the inelastic mechanism that is dominant for sufficiently low temperatures and assume first the regime of overlapping LLs. According to Sec. III.B.2, the linear resistivity is negative when the dimensionless microwave power satisfies $\mathcal{P}>\mathcal{P}^{*}>0$, with the threshold value given by (Dmitriev et al., 2004, 2005)

$$
\mathcal{P}^{*}=\frac{\tau}{\tau_{\text {in }}}\left(4 \delta^{2} \frac{\pi \omega}{\omega_{c}} \sin \frac{2 \pi \omega}{\omega_{c}}-\sin ^{2} \frac{\pi \omega}{\omega_{c}}\right)^{-1},
$$

see Fig. 14. The spontaneous field in the ZRS domains is found to be

$$
E_{c}=E_{0} \sqrt{\frac{\mathcal{P}}{\mathcal{P}^{*}}-1}, \quad E_{0}=\frac{\omega_{\mathrm{c}}}{\pi e R_{c}} \sqrt{\frac{\tau}{2 \tau_{\mathrm{in}}}} .
$$

For the marked MIRO minima in Fig. 14, the corresponding values $E^{*} / E_{0}$ are shown by arrows in the inset.

In the regime of separated LLs with width $2 \Gamma=$ $2\left(2 \omega_{c} / \pi \tau_{\mathrm{q}}\right)^{1 / 2}$ and assuming a small-angle impurity scattering, the threshold power is

$$
\mathcal{P}^{*} \sim \Gamma^{2} \tau / \omega \omega_{c} \tau_{\text {in }},
$$

and the spontaneous field is of the order of

$$
E_{c} \sim\left(\frac{\tau}{\tau_{\mathrm{q}}}\right)^{1 / 2} \frac{\omega_{c}}{e R_{c}}
$$

Within the displacement mechanism (relevant for higher temperatures) and for a small-angle impurity scattering the corresponding results were obtained by Vavilov and Aleiner (2004). In this case the characteristic value of the spontaneous field is given by Eq. (136) in limits of both overlapping and separated LLs.

We point out that the form $\left(\tau / \tau_{\mathrm{q}}\right)^{1 / 2}$ of the factor in front of $\omega_{c} / e R_{c}$ in Eq. (136) depends on the character of disorder. For a model of mixed disorder (93) and for overlapping LLs this factor is reduced and becomes of order unity when the weight of the short-range component 
becomes sufficiently large, see (Dmitriev et al., 2009b; Khodas and Vavilov, 2008) and Sec. III.B, A related result was obtained by Auerbach and Pai (2007), who studied the displacement mechanism for mixed disorder (with short-range component determining the transport rate) in the regime of separated LLs and found $\mathcal{E}_{\mathrm{dc}}^{*} \sim \Gamma / e R_{c}$ (if one sets the correlation length of short-range disorder to be $\sim k_{F}^{-1}$ ). To our knowledge, the inelastic mechanism has not been studied for the case of separated LLs and mixed disorder.

These predictions can be confronted with the experiment. Focusing on the inelastic mechanism and on the regime of overlapping LLs and assuming characteristic values of parameters $\omega / 2 \pi \simeq 50 \mathrm{GHz}, T \sim 1 \mathrm{~K}$, and $\tau_{\mathrm{in}}^{-1} \sim 10 \mathrm{mK}, \tau_{\mathrm{q}}^{-1} \sim 0.3 \mathrm{~K}, \tau^{-1}=10 \mathrm{mK}$, and $v_{F}=2 \cdot 10^{7} \mathrm{~cm} / \mathrm{s}$, one gets (Dmitriev et al., 2004, 2005) the threshold microwave intensity required for the emergence of the ZRS $P^{*} \lesssim 1 \mathrm{~mW} / \mathrm{cm}^{2}$, in conformity with the experiments. Further, for these parameter values and for $\mathcal{P}-\mathcal{P}^{*} \simeq \mathcal{P}^{*}$ (i.e. for the microwave power exceeding its threshold by roughly a factor of 2) the estimated dc electric field in the domains, Eq. (134), is found to be $E_{c} \sim 1 \mathrm{~V} / \mathrm{cm}$. In the experiment of Willett et al. (2004) the voltage drop between an internal and an external contact (separated by $200 \mu \mathrm{m}$ ) generated by the radiation in the absence of the drive current was of the order of $5 \mathrm{mV}$ for $\omega_{c} / 2 \pi \simeq 20 \mathrm{GHz}$. Assuming the simplest domain geometry (i.e no additional domain walls between the contacts), this yields $E_{c} \sim 0.25 \mathrm{~V} / \mathrm{cm}$. On the other hand, the above theoretical estimate yields $E_{c} \sim 0.15 \mathrm{~V} / \mathrm{cm}$ [taking into account the $\omega_{c}^{2}$ dependence of $E_{c}$ following from Eq. (134)], so that the experimental value is somewhat larger. In a more recent experiment (Dorozhkin et al., 2011b) spontaneous fields of the order of $E_{c} \sim 0.15 \mathrm{~V} / \mathrm{cm}$ were reported for $\omega_{c} / 2 \pi \simeq 50 \mathrm{GHz}$, which is in this case several times smaller than the theoretical estimate. In general, the agreement between theory and experiment appears to be very reasonable if one takes into account some deviations of parameters (relaxation rates, ratio of the microwave power to its threshold value) from those used in the theoretical estimate.

\section{Effective theories of the phase transition into ZRS and of ZRS dynamics}

The transition into ZRS belongs to the class of dynamical phase transitions (Hohenberg and Halperin, 1977). These phenomena are in general governed by nonlinear differential equations (of hydrodynamic type) with stochastic (noise) terms and encompass spontaneous pattern formation in a great variety of systems driven away from equilibrium (Cross and Hohenberg, 1993). Contrary to conventional (thermodynamic) phase transitions, such phenomena in general are not characterized by a free energy functional. This invalidates, in particular, the Mermin-Wagner theorem, opening the way to spontaneous breaking of a continuous symmetry in $2 \mathrm{D}$ systems, which is of direct relevance to the ZRS problem.

Auerbach et al. (2005); Finkler et al. (2006) performed an analysis of possible domain patterns in the ZRS phase. In spirit of Sec. IV.B. they started with a model with a constant Hall conductivity $\sigma_{H}$ and a non-linear field dependence of the dissipative current $j_{d}$, Eqs. (126)-(129). Their crucial observation is that for this problem one can define a Lyapunov functional

$$
G[\phi]=\int d^{2} r g(\mathbf{E}(\mathbf{r})), \quad g(\mathbf{E})=\int_{0}^{\mathbf{E}} d \mathbf{E}^{\prime} \mathbf{j}_{d}\left(\mathbf{E}^{\prime}\right),
$$

with the key property $d G / d t \leq 0$. This implies that minima of the Lyapunov functional are stable steady states. To explore the ZRS phase (negative linear dissipative conductivity), Auerbach et al. (2005); Finkler et al. (2006) expand $g(\mathbf{E})$ near the zero $E_{c}$ of the currentvoltage characteristics $j_{d}(E)$ :

$$
g(\mathbf{E})=g\left(E_{c}\right)+\frac{\sigma_{c}}{2}\left(E-E_{c}\right)^{2}+\kappa|\nabla \cdot \mathbf{E}|^{2} .
$$

The last term here [penalizing large field gradients and determining the width of domain walls, $\left.l_{\mathrm{dw}} \sim\left(\kappa / \sigma_{c}\right)^{1 / 2}\right]$ is added on phenomenological grounds. On the microscopic level, a finite width of the domain wall emerges when one takes into account the diffusive contribution $-e \hat{D} \nabla n_{e}$ to the current $j$. However, the local form of the corresponding term in Eq. (138) correspond to the relation between $E$ and $n_{e}$ characteristic for $3 \mathrm{D}$ rather than for $2 \mathrm{D}$ systems, see the discussion at the end of Sec. IV.B.1 It remains to be seen, to what extent this may affect the results.

Minimization of the Lyapunov functional yields the simple domain structures in the Corbino and Hall bar geometries, see Figs. 27 and 28a. Introducing further a weak and smooth disorder field $\mathbf{E}_{\text {dis }}(\mathbf{r})$, with $\left|\mathbf{E}_{\text {dis }}\right| \ll E_{c}$, Auerbach et al. (2005); Finkler et al. (2006) generalized Eq. (138) [denoted below as $\left.g_{0}(\mathbf{E})\right]$ to

$$
g\left(\mathbf{E}, \mathbf{E}_{\mathrm{dis}}\right)=g_{0}(\mathbf{E})-\sigma_{1}(E) \mathbf{E E}_{\mathrm{dis}}+O\left(E_{\mathrm{dis}}^{2}\right) .
$$

The term proportional to $\mathbf{E}_{\text {dis }}$ induces a correction to the stability condition, with the result that the current density at a domain wall is $\mathbf{j}=-\sigma_{1}\left(E_{c}\right) \mathbf{E}_{\text {dis }}+O\left(E_{\text {dis }}^{2}\right)$. For a short correlation length of the disorder a comparison of the domain wall and bulk contribution to the Lyapunov functional in the spirit of Imry and Ma (1975) shows (Auerbach et al., 2005) that no new domains are formed. On the other hand, disorder with a sufficiently large correlation length $\xi_{\text {dis }}$ favors the breakdown of the system in multiple domains with a size set by $\xi_{\text {dis }}$. Further, Auerbach et al. (2005); Finkler et al. (2006) used this approach to estimate the effect of the potential $\phi_{\text {dis }}(\mathbf{r})$ (regular or random) that induces multiple domain walls on the ZRS conductivity. They obtained $\sigma=C \sigma_{1}\left(\left\langle\left|\mathbf{E}_{\text {dis }}\right|\right\rangle\right) / E_{c}$, with a numerical prefactor $C \sim 1$ depending on the specific form (or statistics) of the potential. 

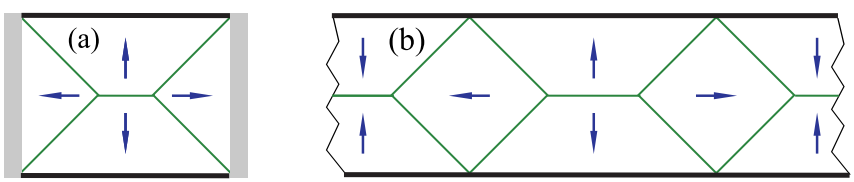

FIG. 28 (a) Proposed domain structure near metallic contacts (shaded grey regions) in a rectangular sample; (b) possible structure with an additional pair of domains. Arrows show the direction of the electric field $\boldsymbol{E}_{c}$, see also Fig.27. Adapted from Finkler et al. (2006); Finkler and Halperin (2009).

Finkler and Halperin (2009) found that even in a homogeneous system creating additional domains may be favorable. They pointed out that the Lyapunov "energy" $\epsilon_{\mathrm{dw}}$ of the domain wall depends on the angle $\phi$ between the wall and the field in the domains. If $\epsilon_{\mathrm{dw}}(\pi / 4) / \epsilon_{\mathrm{dw}}(\pi / 2) \leq 1 / 2 \sqrt{2}$, additional domains as shown in Fig. 28b will emerge. The dependence $\epsilon_{\mathrm{dw}}(\phi)$ is determined by the precise form of the Lyapunov function $g(E)$. Remarkably, for the simplest choice of this function Finkler and Halperin (2009) obtain for the ratio $\epsilon_{\mathrm{dw}}(\pi / 4) / \epsilon_{\mathrm{dw}}(\pi / 2)$ exactly the value $1 / 2 \sqrt{2}$, which implies degeneracy between the states with and without additional domains with $\pi / 4$ domain walls. They conjecture that a more realistic Lyapunov function might yield a smaller value of this ratio, thus favoring additional domains. Finkler and Halperin (2009) consider further the effect of spatial variation of the Hall conductivity $\sigma_{H}$ and show that in this situation non-stationary (periodic) solutions may emerge. The simplest example is the Corbino geometry with Hall conductivity changing linearly along the $y$ axis (radial coordinate), $d \sigma_{H} / d y=\alpha$. Then $\boldsymbol{\nabla} \cdot \mathbf{j}_{H}=\alpha E_{x}$. Combining this with $j_{d}=0$ (characteristic for a homogeneous system away from domain walls), we get $\partial n / \partial t=-\boldsymbol{\nabla} \cdot \mathbf{j}=-\alpha E_{x}(\mathbf{r})$. In the simplest domain geometry (two domains with electric field in the radial direction, Fig. 27) $E_{x}=0$ and we do not get any time dependence. However, in the presence of additional $\pi / 4$ domains, Fig. 28 $\mathrm{b}$, the field there is along the $x$ axis, which implies that such domains should move. Finkler and Halperin (2009) also performed a numerical modeling of the problem in the torus geometry and with a spatially varying Hall conductivity and indeed found nonstationary, time-periodic solutions, in a certain range of the parameter $\alpha$ controlling a typical gradient of $\sigma_{H}$.

What is the character of the transition into the ZRS phase? For equilibrium phase transitions the answer to such a question is usually based on the Landau (meanfield) theory of phase transitions complemented by a renormalization group ( $\mathrm{RG}$ ) analysis. A counterpart of this approach applicable to dynamical critical phenomena (including those in strongly non-equilibrium systems) is known as the dynamical renormalization group (Forster et al., 1977; Hohenberg and Halperin, 1977). A powerful framework for its technical implementation is the Martin-Siggia-Rose formalism (De Dominicis, 1976; De Dominicis and Peliti, 1978; Janssen, 1976;
Martin et al., 1973) closely related to the Keldysh formalism. This approach allows one to cast the evolution governed by a stochastic non-linear equation into a Lagrangian form convenient for the implementation of the RG procedure.

Alicea et al. (2005) made a first step in application of these ideas to the problem of ZRS transition. They started with formulating a general equation describing the long-scale, long-time dynamics of a microwave-driven system:

$$
\begin{aligned}
\partial_{t} \mathbf{j}+ & \omega_{0}^{-1} \partial_{t}^{2} \mathbf{j} \\
= & -\mu \boldsymbol{\nabla} \phi+\omega_{c} \hat{\mathbf{z}} \times \mathbf{j}-r \mathbf{j}-u|\mathbf{j}|^{2} \mathbf{j} \\
& +\eta_{1} \nabla^{2} \mathbf{j}+\eta_{2} \boldsymbol{\nabla}(\boldsymbol{\nabla} \cdot \mathbf{j})-\eta_{3} \nabla^{4} \mathbf{j}-\eta_{4} \boldsymbol{\nabla}^{3}(\boldsymbol{\nabla} \cdot \mathbf{j}) \\
& -\nu_{1}(\mathbf{j} \cdot \boldsymbol{\nabla}) \mathbf{j}-\nu_{2} \boldsymbol{\nabla}\left(\mathbf{j}^{2}\right)-\nu_{3}(\boldsymbol{\nabla} \cdot \mathbf{j}) \mathbf{j} \\
& +\gamma_{1} \phi \boldsymbol{\nabla} \phi+\gamma_{2} \phi \mathbf{j}+\gamma_{3} \phi \hat{\mathbf{z}} \times \mathbf{j}+\boldsymbol{\zeta}+\ldots
\end{aligned}
$$

Here the $\mu$ and $\omega_{c}$ terms represent the electric-field and Lorentz forces, the $r$ and $u$ terms describe the non-linear resistivity, the term with $\partial_{t}^{2}$ (on the left-hand side) originates from the frequency dispersion of the resistivity, the $\eta_{i}$ terms characterize its momentum dispersion, the $\nu_{i}$ terms are convective non-linear contributions, and the $\gamma_{i}$ terms account for the density dependence of transport coefficients. Finally, $\boldsymbol{\zeta}$ is the Langevin noise source with a correlation function

$$
\left\langle\zeta_{\alpha}(\mathbf{r}, t) \zeta_{\beta}\left(\mathbf{r}^{\prime}, t^{\prime}\right)\right\rangle=2 g \delta_{\alpha \beta} \delta\left(\mathbf{r}-\mathbf{r}^{\prime}\right) \delta\left(t-t^{\prime}\right)
$$

Equation (140) is supplemented by the continuity equation (127) and the relation (129) between the potential and the density. Alicea et al. (2005) postulate Eq. (140) on symmetry grounds: it includes leading terms of the expansion in gradients, time derivative, and amplitudes of the current and the potential. It should be pointed out that the assumption (Alicea et al., 2005) of local relation between $\mathbf{j}$ and $\phi$ appears to be an oversimplification, since there are contributions to $\partial_{t} \mathbf{j}(\mathbf{r}, t)$ that depend in a local way on the density $n_{e}(\mathbf{r})$ and since Eq. (129) linking the density to the potential is non-local. It remains to be seen how sensitive is the result to this assumption.

Equation (140) describes a transition from a conventional resistive state at $r>0$ to the ZRS at $r<0$. Within the transition picture of Sec. IV.B. the transition is continuous (second order), and the spontaneous current in the symmetry-broken phase is $j=\sqrt{|r| / u}$, i.e. $j \sim|r|^{\beta}$ with the critical index $\beta=1 / 2$. Clearly, this is just the Landau mean-field description of the transition, and one needs to find out how fluctuations affect these results. This is what Alicea et al. (2005) do; their main findings are as follows:

(i) The symmetry-broken state is stable with respect to current-density fluctuations (there is no infrared divergence contrary to usual thermodynamic transitions where the Mermin-Wagner theorem implies a logarithmic divergence in $2 \mathrm{D}$ leading to destruction of the long-range order). 
(ii) For the model with short-range interaction, $U(\mathbf{r}-$ $\left.\mathbf{r}^{\prime}\right)=C^{-1} \delta\left(\mathbf{r}-\mathbf{r}^{\prime}\right)$ (that would correspond to a system with a screening gate) and with a symmetry with respect to $\phi \rightarrow \phi+$ const the Gaussian fixed point of the mean-field theory is stable, with non-linear terms being marginally irrelevant. As a result, the meanfield exponents hold, implying the scaling $j \sim|r|^{\beta}$ with $\beta=1 / 2$ on the ZRS side of the transition, as well as $j \sim|E|^{\delta}$ with $\delta=1 / 3$ and the overdamped "diffusion mode" $\sim 1 /\left(-i \omega+D q^{4}\right)$ at criticality, with logarithmic corrections to scaling. The invariance with respect to $\phi \rightarrow \phi+$ const forbids in Eq. (140) those terms that explicitly depend on the value of $\phi(\mathbf{r})$ (rather than on its gradients). In the physical system such invariance does approximately hold; however, it gets violated when variations of density [linked to those of $\phi$ via Eq. (129)] become comparable to the total density of the electron gas.

(iii) The long-range Coulomb interaction drives the system away from the Gaussian fixed point. Alicea et al. (2005) analyze the RG equations in the model with $1 / k^{\epsilon}$ interaction (in momentum space) that has the upper critical dimension $d=2+\epsilon$ and find no stable fixed points in $2 \mathrm{D}$. This leads them to the conclusion that the transition becomes first order in the presence of long-range interaction. Presumably, this should also hold in the physical case of $1 / k$ interaction, i.e. $\epsilon=1$.

(iv) The terms not included in the $\phi \rightarrow \phi+$ const model drive the transition first order as well (whether with short-range or with long-range interaction). These results are obtained by the analysis of the RG flow $d_{\mathrm{UC}}-\epsilon$ dimensions, where the upper critical dimension $d_{\mathrm{UC}}$ is found to be $d_{\mathrm{UC}}=4$ and $d_{\mathrm{UC}}=7$ for short- and longrange interactions, respectively. In both cases no stable fixed points are found, implying that the transition gets first order. This conclusion is then extrapolated to the physical dimension $d=2$.

(v) Alicea et al. (2005) also point out a similarity between the ZRS problem and a phase transition in the $2 \mathrm{D}$ bird-flocking model. The latter is essentially a dynamical generalization of the 2D $X Y$-model: birds move with a constant velocity and at each time step every of them picks up a new direction governed by average velocity of surrounding birds, with some level of noise (Grégoire and Chaté, 2004; Toner and Tu, 1995, 1998; Vicsek et al., 1995). For a sufficiently weak noise the rotational symmetry gets spontaneously broken: the bird flock acquires a macroscopic collective velocity. While Vicsek et al. (1995) found a second-order phase transition, a more recent work (Grégoire and Chaté, 2004) obtains a first-order transition. (Alicea et al., 2005) find that the ZRS and the bird-flocking model are almost equivalent, up to the Lorentz-force term (present in the former but not in the latter), so that the first-order transitions in both of them would be mutually consistent.

\section{HALL FIELD- AND PHONON-INDUCED RESISTANCE OSCILLATIONS (HIRO AND PIRO)}

\section{A. HIRO: Experimental discovery and basic properties}

A decade ago Yang et al. (2002a) discovered prominent $1 / B$-oscillations of the differential resistivity $r \equiv$ $\rho+I(d \rho / d I)$ in a 2DEG with moderate mobility $\mu \sim$ $10^{6} \mathrm{~cm}^{2} / \mathrm{V} \mathrm{s}$ subject to a constant direct current $I$, see Fig. 29a. These oscillations, termed the Hall fieldinduced resistance oscillations (HIRO), can alternatively be observed at fixed $B$ and varying $I$. The latter approach allows one to observe more oscillations since the amplitude of the DOS modulation (fixed by the value $\left.\omega_{\mathrm{c}} \tau_{\mathrm{q}}\right)$ does not change during the measurement. Examples of both realizations of HIRO in a high mobility $\left(\mu \sim 10^{7} \mathrm{~cm}^{2} / \mathrm{Vs}\right) 2 \mathrm{DEG}$ are shown in Fig.29 and c. Yang et al. (2002a) proposed that HIRO stem
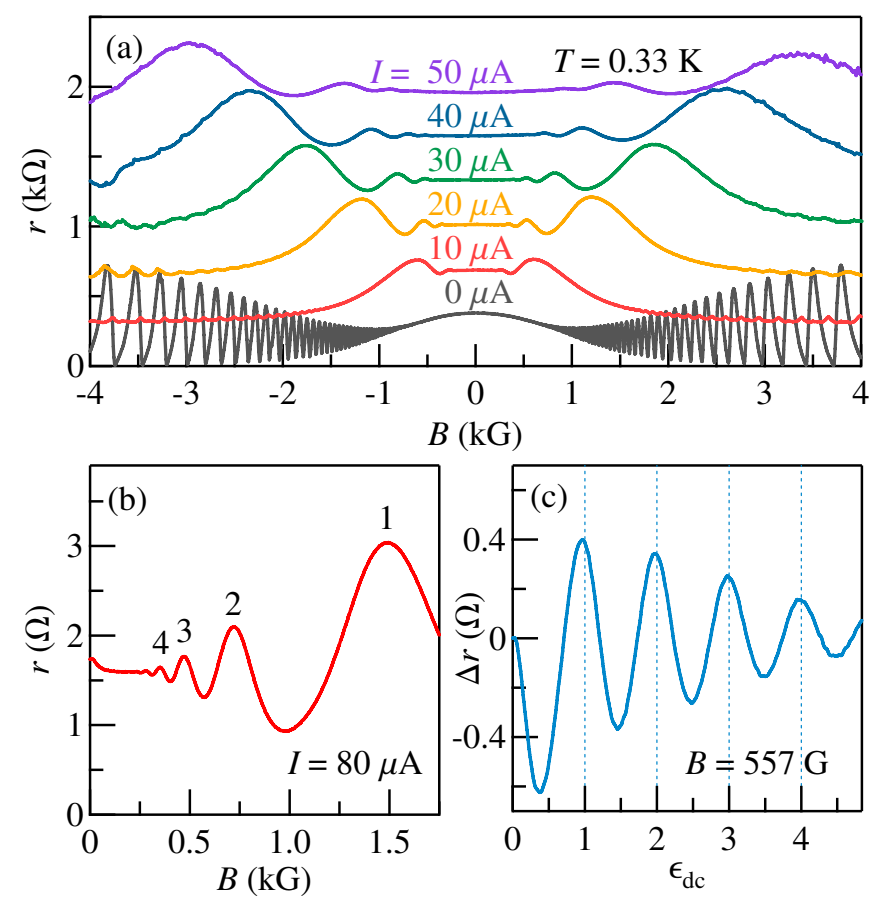

FIG. 29 (a) Differential magnetoresistance $r(B)$ measured at different dc current $I$, as marked, in a Hall bar sample of width $w=50 \mu m$ with $\mu \simeq 3.0 \times 10^{6} \mathrm{~cm}^{2} / \mathrm{V} \mathrm{s}$ and $n_{e} \simeq 2.0 \times 10^{11}$ $\mathrm{cm}^{-2}$. Adapted from Yang et al. (2002a). (b) Differential magnetoresistivity $r$ showing four HIRO maxima marked by integers measured by sweeping $B$ at fixed $I=80 \mu \mathrm{A}$. (c) Correction to the differential resistivity $\delta r$ versus $\epsilon_{\mathrm{dc}}$ obtained by sweeping $I$ at fixed $B=557 \mathrm{G}$. The data were obtained at $T \simeq 1.5 \mathrm{~K}$ in a $100 \mu \mathrm{m}$ wide Hall bar sample with $n_{e} \simeq$ $3.7 \times 10^{11} \mathrm{~cm}^{-2}$ and $\mu \simeq 1.2 \times 10^{7} \mathrm{~cm}^{2} / \mathrm{V} \mathrm{s}$. Adapted from Zhang et al. (2007b).

from the commensurability between the cyclotron diameter $2 R_{\mathrm{c}}$ and the spatial separation $\Delta y=\omega_{\mathrm{c}} / e E$ between the Hall field-tilted LLs. Here $E=\rho_{H} j$ is the Hall electric field, $j=I / w$ is the current density, and $w$ is the Hall bar width. This yields oscillations with 
$\epsilon_{\mathrm{dc}}=2 R_{\mathrm{c}} / \Delta y=2 e E R_{\mathrm{c}} / \omega_{\mathrm{c}}$ with a characteristic dependence $\epsilon_{\mathrm{dc}} \propto n_{e}^{-1 / 2}$ on the electron density, confirmed by Yang et al. (2002a). In addition to single-layer 2D electron systems, HIRO were also observed in double QWs (Bvkov, 2008c; Mamani et al., 2009a; Wiedmann et al., 2011a) and in 2D hole systems based on carbon-doped GaAs/AlGaAs QWs (Dai et al., 2009). HIRO were reported to retain their dc character under ac excitation with frequencies up to $100 \mathrm{kHz}$ (Bykov et al., 2005b). Very recently HIRO were also observed in a Corbino ringshaped 2DEG (Bykov et al., 2012).

At $2 \pi \epsilon_{\mathrm{dc}} \gtrsim 1$, the oscillatory part $\Delta r$ of HIRO in Fig. 29 is well described (Zhang et al., 2007b) by

$$
\Delta r / \rho \simeq a \delta^{2} \cos \left(2 \pi \epsilon_{\mathrm{dc}}\right)
$$

In agreement with other experiments (Hatke et al., 2010, 2009b, 2011f), the HIRO maxima and minima in Fig.29 occur at integer and half-integer values of $\epsilon_{\mathrm{dc}}$, respectively. The amplitude $a$, obtained by Vavilov et al. (2007) for $\epsilon_{\mathrm{dc}} \gg 1$ in the form

$$
a=16 \tau / \pi \tau_{\pi},
$$

is proportional to the backscattering rate $1 / \tau_{\pi}$, see Eq. (150) below. HIRO can thus be understood in terms of resonant inter-LL transitions resulting from backscattering off impurities, i.e., HIRO require a sufficient amount of short-range scatterers for their observation. Dingle-plot analysis using Eqs. (142), (143) allows one to extract both the quantum lifetime $\tau_{\mathrm{q}}$ and the backscattering time $\tau_{\pi} \simeq \tau_{\mathrm{sh}}$. For a $2 \mathrm{DEG}$ with $\mu \sim 10^{7} \mathrm{~cm}^{2} / \mathrm{V} \mathrm{s}$, such analysis at low $T$ produced $\tau_{\mathrm{q}} \simeq 20 \mathrm{ps}$ and $\tau_{\pi} \simeq 5 \tau$ (Hatke et al., 2009b).

Hatke et al. (2009b) demonstrated that, similar to MIRO, the decay of HIRO at high $T$ is primarily due to the LL broadening induced by electron-electron interactions, which gives $\delta^{2}=e^{-2 \pi / \omega_{\mathrm{c}} \tau_{\mathrm{q}}^{*}}$ in Eq. (142) with $1 / \tau_{\mathrm{q}}^{*}(T)-1 / \tau_{\mathrm{q}} \propto T^{2}$, see Secs. III.A. III.B.1.f. At low $T$, the experimentally observed $T$ dependence of $1 / \tau_{\mathrm{q}}^{*}(T)$ deviates from the $T^{2}$ law, which can be related to the heating of $2 \mathrm{DEG}$ by the direct current. The heating may also explain the decay of HIRO with $\epsilon_{\mathrm{dc}}$ at constant $B$, see Fig.29b. According to Eq. (142), the decay likely results from a suppression of $\delta$ as $I$ increases.

Hatke et al. (2011f) found that the HIRO amplitude is strongly suppressed [similar to MIRO (Yang et al., 2006) (see Sec.[II.C.3)] by the in-plane magnetic field $B_{\|} \sim 1 \mathrm{~T}$, while the HIRO period depends only on the perpendicular component. The experiment demonstrates that the suppression should be attributed to a $B_{\|}$-induced enhancement of the quantum scattering rate $1 / \tau_{\mathrm{q}}^{*}-1 /\left.\tau_{\mathrm{q}}^{*}\right|_{B_{\|}=0} \propto B_{\|}^{2}$. In the regime of strongly developed $\mathrm{SdH}$ oscillations, $B_{\|}$starts to manifest itself also via the Zeeman splitting, see Sec.VII.A.5.

\section{B. Theory of nonlinear dc transport}

Similar to the mechanisms of MIRO (Sec. III.B), two most important contributions to the nonlinear electric current in the absence of microwaves come from the direct effect of the dc field on impurity scattering (displacement mechanism) and from nonequilibrium changes of the isotropic part of the distribution function (inelastic mechanism). For smooth disorder, these effects were systematically studied by Vavilov and Aleiner (2004) (displacement mechanism) and by Dmitriev et al. (2005) (inelastic). A comprehensive analysis for a generic model of disorder [Eq. (71)] for high $T \gg \omega_{\mathrm{c}}$ and overlapping LLs was performed in (Vavilov et al., 2007).

Next we provide an overview of the main results of these works using the formulation of Sec. III.B.1 (which, in turn, follows from the general quantum kinetic approach outlined in Sec. III.B.3). In the absence of the microwave field, Eqs. (65)-(68) reduce to

$$
j_{d}=2 \nu_{0} e \int d \varepsilon\left\langle\Delta X_{\varphi \varphi^{\prime}} \tau_{\varphi-\varphi^{\prime}}^{-1} \tilde{\nu}_{\varepsilon} \tilde{\nu}_{\varepsilon^{\prime}}\left(f_{\varepsilon}-f_{\varepsilon^{\prime}}\right)\right\rangle_{\varphi \varphi^{\prime}},
$$

where $\varepsilon^{\prime}=\varepsilon+e E \Delta X_{\varphi \varphi^{\prime}}$ and the angle brackets denote averaging over $\varphi$ and $\varphi^{\prime}$. The isotropic part $f$ of the distribution function obeys

$$
\left(f_{\varepsilon}-f_{\varepsilon}^{T}\right) / \tau_{\text {in }}=\left\langle\tau_{\varphi-\varphi^{\prime}}^{-1} \tilde{\nu}_{\varepsilon^{\prime}}\left(f_{\varepsilon}-f_{\varepsilon^{\prime}}\right)\right\rangle_{\varphi \varphi^{\prime}} .
$$

We restrict further analysis to $2 \pi^{2} T / \omega_{\mathrm{c}} \gg 1$ (thermally suppressed $\mathrm{SdH}$ oscillations) and $\omega_{\mathrm{c}} \tau_{\mathrm{q}} \ll 1$ (overlapping LLs). In this limit, Eqs. (144) and (145) yield the main result of (Vavilov et al., 2007)

$$
\frac{j_{d}}{\sigma_{\mathrm{D}} E}=1-2 \delta^{2} \tau \gamma^{\prime \prime}(\zeta)+2 \delta^{2} \tau \frac{\gamma^{\prime}(\zeta)}{\zeta} \mathcal{F}\left(\tau_{\text {in }}, \zeta\right),
$$

where $\gamma(\zeta)$, for $\tau_{\varphi-\varphi^{\prime}}^{-1}$ parametrized by Eq. (71), reads

$$
\gamma(\zeta)=\sum_{n} \tau_{n}^{-1} J_{n}^{2}(\zeta), \quad \zeta \equiv \pi \epsilon_{\mathrm{dc}}=\frac{2 \pi e E R_{c}}{\omega_{\mathrm{c}}},
$$

with $J_{n}(\zeta)$ being the Bessel functions. The second (displacement) term in Eq. (146) comes from Eq. (144) when substituting the equilibrium distribution $f_{\varepsilon}^{T}$ for $f_{\varepsilon}$. The last (inelastic) term is proportional to the amplitude

$$
\mathcal{F}\left(\tau_{\mathrm{in}}, \zeta\right)=\frac{-2 \zeta \gamma^{\prime}(\zeta)}{\tau_{\mathrm{in}}^{-1}+\tau_{0}^{-1}-\gamma(\zeta)}
$$

of the oscillations of $f_{\varepsilon}$ in Eq. (145),

$$
f_{\varepsilon}=f_{\varepsilon}^{T}+\delta \sin \left(\frac{2 \pi \varepsilon}{\omega_{\mathrm{c}}}\right) \mathcal{F}\left(\tau_{\mathrm{in}}, \zeta\right) \frac{\omega_{\mathrm{c}}}{2 \pi} \partial_{\varepsilon} f_{\varepsilon}^{T} .
$$

In the large- $\zeta$ limit, Eq. (146) is significantly simplified:

$$
j_{d} \rightarrow \sigma_{\mathrm{D}} E+2 \delta^{2} \sigma_{\mathrm{D}} E \frac{4 \tau}{\pi \tau_{\pi} \zeta} \sin 2 \zeta,
$$


where the backscattering rate $\tau_{\pi}^{-1}=\sum_{n} \tau_{n}^{-1} \exp (i n \pi)$ (the actual condition on $\zeta$ depends on the type of disorder, see below). The nonlinear current is then dominated by the displacement term and shows oscillations proportional to $\sin 2 \zeta$, which yields the $\cos 2 \zeta$ dependence of the differential resistivity observed in the experiment, cf. Eq. (142).

To explain the HIRO phase, let us look at Eq. (144) for $T \gg \omega_{\mathrm{c}} \zeta \sim e E R_{c}$ and neglect the oscillations of $f_{\varepsilon}$ by assuming $f_{\varepsilon}=f_{\varepsilon}^{T}$. Then, Eq. (144) is expressible as an energy averaging over the period $\omega_{\mathrm{c}}$ :

$$
\frac{j_{d}}{2 \nu_{0} e^{2} E}=\left\langle\frac{\left(\Delta X_{\varphi \varphi^{\prime}}\right)^{2}}{\tau_{\varphi-\varphi^{\prime}}}\left\langle\tilde{\nu}_{\varepsilon} \tilde{\nu}_{\varepsilon+e E \Delta X_{\varphi \varphi^{\prime}}}\right\rangle_{\varepsilon}\right\rangle_{\varphi \varphi^{\prime}} .
$$

In overlapping LLs, $\left\langle\tilde{\nu}_{\varepsilon} \tilde{\nu}_{\varepsilon+\omega}\right\rangle_{\varepsilon}=1+2 \delta^{2} \cos \left(2 \pi \omega / \omega_{\mathrm{c}}\right)$. The angular integrations in Eq. (151) within the stationary-phase approximation in the limit $E \rightarrow \infty$ yield Eq. (150). The main contribution to HIRO comes from the backscattering processes with $\varphi \simeq \pm \pi / 2$ and $\left|\varphi-\varphi^{\prime}\right| \simeq \pi$. These correspond to the maximum possible shift $\Delta X_{\varphi \varphi^{\prime}} \simeq 2 R_{c}$ along the electric field. The average $\left\langle\tilde{\nu}_{\varepsilon} \tilde{\nu}_{\varepsilon+2 e E R_{c}}\right\rangle_{\varepsilon}$ is maximized at integer $\epsilon_{\mathrm{dc}}=2 e E R_{c} / \omega_{\mathrm{c}}$, which results in HIRO maxima in the differential resistivity at integer $\epsilon_{\mathrm{dc}}$, cf. Eq. (142).

The amplitude of the resistivity oscillations in Eq. (150) does not depend on $E$ and $T$, while experiments show considerable suppression for strong current and/or high T. As discussed in Sec.V.A this suppression can be explained as a result of additional broadening of LLs by electron-electron interactions, combined with the effect of heating of the 2DEG by the dc field which is controlled by electron-phonon interactions.

To analyze the obtained results, Vavilov et al. (2007) introduced the mixed-disorder model (93) (used in Sec. III.B to describe MIRO). The smooth (characterized by $\left.\tau_{\mathrm{q}, \mathrm{sm}}^{-1}\right)$ and sharp $\left(\tau_{\mathrm{sh}}^{-1}\right)$ components of disorder yield two separate contributions to

$$
\gamma(\zeta)=\frac{J_{0}^{2}(\zeta)}{\tau_{\mathrm{sh}}}+\frac{1}{\tau_{\mathrm{q}, \mathrm{sm}} \sqrt{1+\chi \zeta^{2}}}
$$

The contribution of the sharp component changes on a scale of $\zeta \sim 1$ and shows strong oscillations for $\zeta \gtrsim 1$. The second term describes the nonlinear effects, studied by Vavilov and Aleiner (2004), that result from small-angle scattering by the long-range component ( $\chi^{1 / 2} \ll 1$ gives a typical value of the scattering angle, see Sec. III.B. . These become relevant in a much stronger dc field, $\zeta \sim \chi^{-1 / 2} \gg 1$, and do not contribute to HIRO. At $\zeta \ll 1$, Eqs. (146) and (148) reduce to

$$
\begin{aligned}
& \frac{j_{d}}{\sigma_{\mathrm{D}} E}=1+2 \delta^{2}-\frac{3 \tau}{4 \tau_{\star}} \delta^{2} \zeta^{2}-2 \delta^{2} \mathcal{F}\left(\tau_{\mathrm{in}}, \zeta\right) \\
& \mathcal{F}\left(\tau_{\mathrm{in}}, \zeta\right)=\frac{2 \tau_{\mathrm{in}} \zeta^{2} / \tau}{1+\tau_{\mathrm{in}} \zeta^{2} / 2 \tau}
\end{aligned}
$$

where $1 / \tau_{\star} \simeq 3 / \tau_{\mathrm{sh}}+12 \chi^{2} / \tau_{\mathrm{q}, \mathrm{sm}}$ and $1 / \tau \simeq 1 / \tau_{\mathrm{sh}}+$ $\chi / \tau_{\mathrm{q}, \mathrm{sm}}$. Note that the nonlinear terms $\propto \zeta^{2}$ in Eqs. (153) and (154) can be equivalently obtained by taking the limit of $\omega \rightarrow 0$ in the MIRO terms in Eqs. (77), (82), and (84). At order $E^{3}$ in $j_{d}$, the ratio of the inelastic and displacement contributions in the case of smooth disorder is of the order of $\tau_{\text {in }} \tau_{\star} / \tau^{2} \sim \tau_{\text {in }} / \tau_{\mathrm{q}} \gg 1$ [similar to MIRO, Eq. (100)]. That is, the inelastic term, obtained for smooth disorder by Dmitriev et al. (2005), dominates and Eqs. (153) and (154) are valid for all $\zeta \ll \chi^{-1 / 2}$. The suppression of $j_{d} / E$ in a relatively weak dc field at $\zeta \sim\left(\tau / \tau_{\text {in }}\right)^{1 / 2}$ is the strongest effect in the nonlinear dc response for the case of smooth disorder.

It is important to note the dual role that the electric field plays in Eqs. (148) and (154). On the one hand, it creates the oscillations (149) in the energy distribution of electrons; on the other, it also opens an additional channel of inelastic relaxation, thus controlling the magnitude of the oscillations. Indeed, in the presence of the dc field, an electron can change its kinetic energy via elastic collisions with impurities. At $\zeta \gg\left(\tau / \tau_{\text {in }}\right)^{1 / 2}$, the resulting "spectral diffusion" becomes more efficient than the inelastic relaxation due to electron-electron collisions. This leads to the saturation of the oscillations in Eqs. (154) and (149) and makes the nonlinear response independent of $T$ for $\zeta \gg\left(\tau / \tau_{\text {in }}\right)^{1 / 2}$. Because of the spectral diffusion, $\mathcal{F}\left(\tau_{\text {in }}, \zeta\right)$ remains smaller than or of the order of unity for arbitrary $\zeta$. Note that the oscillatory term in Eq. (149) contains additionally two small factors $\delta$ and $\omega_{\mathrm{c}} \partial_{\varepsilon} f_{\varepsilon}^{T} \sim-\omega_{\mathrm{c}} / T$ and, therefore, remains small at any $\zeta$, which makes the expansion (149) legitimate. Unlike Eq. (153), which contains the "pure" displacement and inelastic contributions, the last term in Eq. (146) is, strictly speaking, a result of the interplay of the inelastic and displacement mechanisms in the strongly nonlinear response.

Let us now consider the case $\chi \tau_{\text {sh }} \ll \tau_{\text {q,sm }} \ll \tau_{\text {sh }}$ and $\zeta \ll \chi^{-1 / 2}$, when the short-range component of disorder determines the transport scattering rate, i.e., $\tau_{\mathrm{sh}} \simeq \tau$, while the nonlinear corrections generated by the smooth component are negligible. Equations (146) and (148) then reduce to

$$
\frac{j_{d}}{\sigma_{\mathrm{D}} E} \simeq 1-2 \delta^{2}\left[J_{0}^{2}(\zeta)\right]^{\prime \prime}-\frac{16 \delta^{2} J_{0}^{2}(\zeta) J_{1}^{2}(\zeta)}{\tau / \tau_{\mathrm{in}}+1-J_{0}^{2}(\zeta)}
$$

In this limit, $\tau_{\mathrm{q}, \mathrm{sm}} \simeq \tau_{\mathrm{q}}$ enters $j_{d}$ only via the Dingle factor $\delta=\exp \left(-\pi / \omega_{\mathrm{c}} \tau_{\mathrm{q}}\right)$. The above expression captures both the high-field limit (150) for HIRO and its essential modifications at intermediate $(\zeta \sim 1)$ and small $(\zeta \sim$ $\sqrt{\tau / \tau_{\text {in }}}$ ) electric fields. Unless $\tau_{\text {in }} \ll \tau$ (which may occur at elevated $T$ ), the inelastic and displacement terms in Eq. (155) are equally important at $\zeta \sim 1$. Provided $\tau_{\mathrm{sh}} \sim$ $\tau$, the smooth component only slightly modifies Eq. (155) in the relevant range of $\zeta \ll \chi^{-1 / 2}$, see Eq. (3.8) and Figs. 2,3 in (Vavilov et al., 2007). 


\section{Nonlinear resistivity: Inelastic effects}

In accordance with the theoretical predictions, the most pronounced inelastic effects in the nonlinear resistivity were observed in 2DEGs with high density $n_{e} \sim 10^{12} \mathrm{~cm}^{-2}$ and moderate mobility $\mu \sim 10^{6} \mathrm{~cm}^{2} / \mathrm{V} \mathrm{s}$ (Bvkov et al., 2005b, 2007; Kalmanovitz et al., 2008; Mamani et al., 2009a; Vitkalov, 2009; Zhang et al., 2009, 2007a). Indeed, according to Eqs. (153) and (154), the differential resistivity at order $\zeta^{2}$ reads

$$
r=\rho_{\mathrm{D}}\left(1+2 \delta^{2}\right)-12 \delta^{2} \rho_{\mathrm{D}}\left(\frac{3 \tau}{16 \tau_{\star}}+\frac{\tau_{\text {in }}}{\tau}\right) \zeta^{2} .
$$

The maximum value of the factor $3 \tau / 16 \tau_{\star}$ in the displacement term is $9 / 16$ (which corresponds to $\tau=\tau_{\text {sh }}$, see Fig.13a), while the factor $\tau_{\text {in }} / \tau$ in the inelastic contribution can be estimated as $\varepsilon_{F} / T^{2} \tau \simeq 130(1 \mathrm{~K} / T)^{2}$ (for $\mu=10^{6} \mathrm{~cm}^{2} / \mathrm{Vs}$ and $n_{e}=10^{12} \mathrm{~cm}^{-2}$ ). This gives $16 \tau_{\star} \tau_{\text {in }} / 3 \tau^{2} \gtrsim(15 \mathrm{~K} / T)^{2}$ for the relative magnitude meaning the inelastic contribution dominates up to high $T \gtrsim 15 \mathrm{~K}$. This justifies the analysis of the low-field nonlinear resistivity performed by Mamani et al. (2009a); Vitkalov (2009); Zhang et al. (2009, 2007a) solely in terms of the inelastic mechanism [and also justifies retaining the higher-order terms in Eq. (154)]. All these experiments reproduced the theoretical predictions in the range of applicability of the theory ${ }^{18}$ and reported values of $\tau_{\text {in }} \propto T^{-2}$ close to those calculated by Dmitriev et al. (2005). In particular, Mamani et al. (2009a) generalized the theory (Dmitriev et al., 2005) to the two-subband case and found that the MISO peaks in the nonlinear response are inverted at $\zeta=\left(2 \tau / 3 \tau_{\text {in }}\right)^{1 / 2}$, which was used to accurately determine $\tau_{\text {in }}$. The effect is similar to the interplay of MISO and MIRO discussed in Sec.III.C.4. In the nonlinear dc response, interaction of MISO and MIRO was observed by Wiedmann et al. (2011a) and explained therein in terms of the inelastic mechanism. The data (Wiedmann et al., 2011a) indicate also a twosubband counterpart of the nonlinear mixing of HIRO and MIRO described in Sec.VI.B.

In the ultra-high mobility $2 \mathrm{DEG}$ with $n_{e} \simeq 3.95 \times$ $10^{11} \mathrm{~cm}^{-2}$ and $\mu \simeq 8.9 \times 10^{6} \mathrm{~cm}^{2} / \mathrm{Vs}$ used by (Hatke et al., 2012a), the estimated ratio $\tau_{\text {in }} / \tau \simeq$ $\varepsilon_{F} / T^{2} \tau \simeq(2 \mathrm{~K} / T)^{2}$ is much smaller than in the samples discussed above and, as a result, both contributions in Eq. (156) are relevant. In this case, the Dingle analysis of the low-field differential resistivity at fixed $T=1.5 \mathrm{~K}$ yielded a value of 2.25 for the expression in the brackets in the nonlinear term of Eq. (156). Using Eq. (94), for

18 The analytical results by (Dmitriev et al., 2005; Mamani et al., 2009a) are applicable for $2 \pi^{2} T / \omega_{\mathrm{c}} \gg 1$ and overlapping LLs. In the regime of $\mathrm{SdH}$ oscillations $\left(2 \pi^{2} T / \omega_{\mathrm{c}} \lesssim 1\right)$, which was also studied experimentally by Bykov et al. (2007); Kalmanovitz et al. (2008); Zhang et al. (2007a), such a description is not parametrically justified and additional theoretical analysis is required, see (Dmitriev, 2011). $\tau=20 \tau_{\mathrm{q}}$ and $\tau_{\mathrm{sh}} \simeq 5 \tau$ (Hatke et al., 2009b) one obtains $3 \tau / 16 \tau_{\star} \simeq 0.23$ which divides almost equally between the sharp and smooth components of disorder, see Fig.13. It follows that the inelastic term $\tau_{\text {in }} / \tau \simeq 2$ at $T=1.5 \mathrm{~K}$, which agrees well with the estimate above. This example shows that measurements of the nonlinear magnetoresistivity in high LLs in both limits $\zeta \gg 1$ (HIRO) and $\zeta \ll 1$ [Eq. (156)] provide a method to determine various scattering rates - in particular, $T$-dependent $\tau_{\mathrm{q}}$ and $\tau_{\text {in }}$, as well as $\tau_{\star}$ and $\tau_{\pi}$-which bring valuable information about disorder and interactions in $2 \mathrm{D}$ electron systems.

\section{Zero-differential resistance states (ZdRS)}

Experiments by Bykov et al. (2007); Zhang et al. (2008b) revealed that the differential resistance can drop all the way to zero (Fig. 30), leading to the formation of the zero-differential resistance states (ZdRS). Bykov et al. (2007); Kalmanovitz et al. (2008) (in samples with $n_{e} \simeq 8 \times 10^{11} \mathrm{~cm}^{-2}$ and $\left.\mu \simeq 8 \times 10^{5} \mathrm{~cm}^{2} / \mathrm{V} \mathrm{s}\right)$ observed ZdRS emerging from the maxima of SdH oscillations. The transition to ZdRS was accompanied by a reproducible negative spike (Fig. [30a) and by temporal fluctuations at higher dc bias.
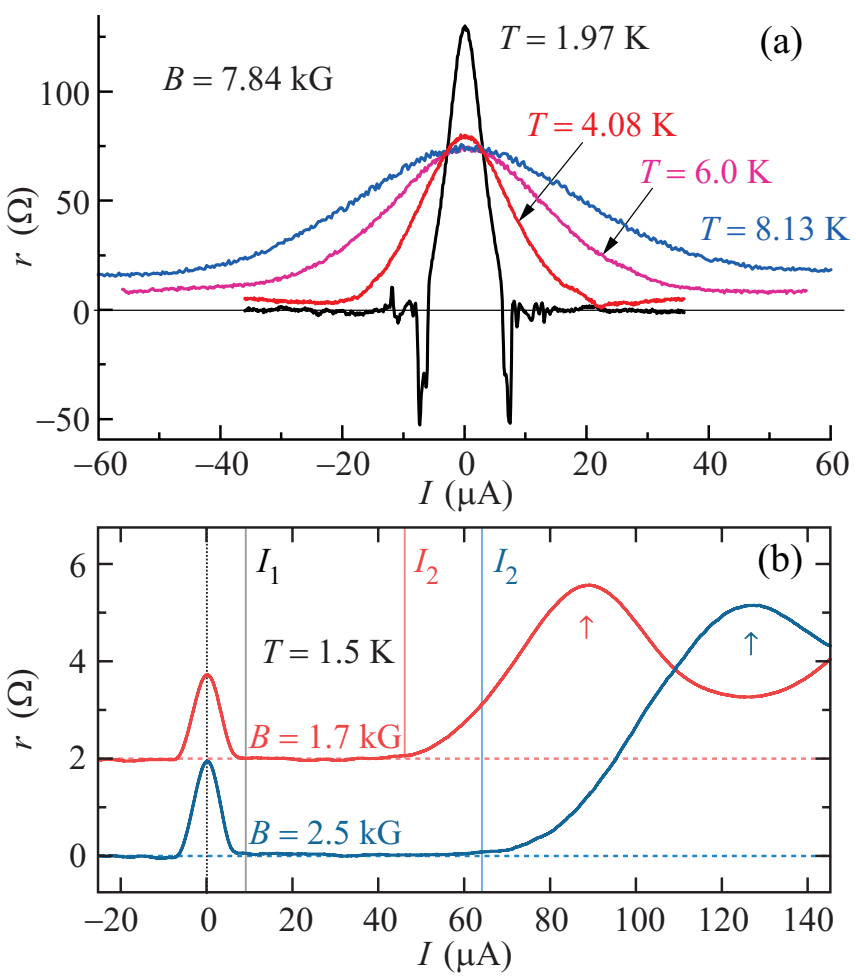

FIG. 30 (a) Differential resistivity $r$ vs current $I$ for several values of $T$ at fixed $B=7.64 \mathrm{kG}$, measured in a $50 \mu \mathrm{m}$ wide Hall bar sample. Adapted from Bvkov et al. (2007). (b) Similar dependence at $B=1.7$ and $B=2.5 \mathrm{kG}$, measured at $T \simeq 1.5 \mathrm{~K}$ in a $100 \mu \mathrm{m}$ wide Hall bar sample. The first HIRO maximum is marked by $\uparrow$. The curve for $B=1.7 \mathrm{kG}$ is shifted by $2 \Omega$ upward. Adapted from Hatke et al. (2010). 
By contrast, Zhang et al. $2008 \mathrm{~b})$ (in a sample with $n_{e} \simeq 4.8 \times 10^{11} \mathrm{~cm}^{-2}$ and $\mu \simeq 4.4 \times 10^{6} \mathrm{~cm}^{2} / \mathrm{V} \mathrm{s}$ ) and Hatke et al. (2010) (in samples with $n_{e} \simeq 3.8 \times 10^{11} \mathrm{~cm}^{-2}$ and $\mu \simeq 1.0 \times 10^{7} \mathrm{~cm}^{2} / \mathrm{V} \mathrm{s}$ ) demonstrated the possibility of ZdRS evolving from the principal minimum of HIRO in the regime of suppressed $\mathrm{SdH}$ oscillations. Neither overshoot to negative values nor temporal fluctuations were detected (Fig.30b). Hatke et al. (2010) reported ZdRS over a continuous range of currents and in magnetic fields extending well below the onset of SdH oscillations. Similar to ZRS, ZdRS were found to disappear with increasing temperature $(T \gtrsim 2 \mathrm{~K})$ and with increasing overlap between LLs $(B \lesssim 1 \mathrm{kG})$. The minimum current $I_{1}$ (Fig. 30 b) required to support ZdRS was found to be roughly $B$-independent. The maximum current $I_{2}$ was found to increase roughly linearly with $B$, tracing the fundamental HIRO peak (cf. $\uparrow$ in Fig.30b). ZdRS were also observed to develop from the maxima of MIRO (Zhang et al., 2007c) and MISO (Bvkov et al., 2010d; Gusev et al., 2011; Wiedmann et al., 2011a).

On the theoretical side, ZdRS originate from the parts of (local) $I-V$ characteristics where the differential resistivity is negative, which violates the stability condition (131). Similar to the case of ZRS (Sec.IV), this results in an instability leading to the formation of current and field domains. Unlike ZRS, however, the absolute resistivity remains positive, which leads to an essential difference between the Corbino (N-shaped $I-V$ characteristics) and Hall bar (S-shaped $I-V$ characteristics) geometries. While in the Hall bar geometry a stationary domain configuration with the current flowing along the domain walls is expected (Bykov et al., 2007), in the Corbino geometry nonstationary (moving) domains are predicted (Vavilov and Aleiner, 2004) similar to the Gunn effect (Gunn, 1963).

\section{E. PIRO: Experimental discovery and basic properties}

Resonant interaction of 2D electrons with longitudinal optical (LO) phonons was predicted (Gurevich and Firsov, 1961) and confirmed in magnetotransport measurements in GaAs/AlGaAs heterostructures (Tsui et al., 1980) long time ago. This interaction manifests itself as an enhancement of the longitudinal resistivity whenever the LO-phonon frequency $\omega_{\mathrm{LO}} \simeq n \omega_{\mathrm{c}}, n=1,2,3, \ldots$ In GaAs, $\omega_{\mathrm{LO}} \sim 10^{13} \mathrm{~s}^{-1}$ and observation of the LO-phonon-induced oscillations requires high $T \gtrsim 10^{2} \mathrm{~K}$ and strong $B \gtrsim 10^{2} \mathrm{kG}$ (Tsui et al., 1980).

A decade ago, another class of phonon-induced oscillations, termed the phonon-induced resistance oscillations (PIRO), was discovered in the linear-response resistivity of a 2DEG with mobility $\mu \sim 10^{6} \mathrm{~cm}^{2} / \mathrm{V} \mathrm{s}$ (Zudov et al. , 2001b). PIRO emerged at much lower $T \sim 1-10 \mathrm{~K}$ and much lower $B \sim 1-10 \mathrm{kG}$ and are understood in terms of resonant interaction of $2 \mathrm{D}$ electrons in high LLs with acoustic phonons which carry momentum $2 k_{F}$ and have a
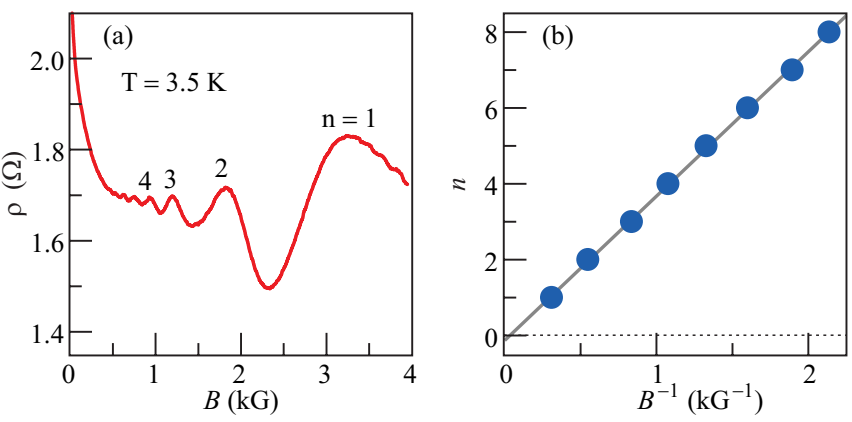

FIG. 31 (a) Magnetoresistivity $\rho$ as a function of $B$, showing eight PIRO maxima, four of which are marked by integers. (b) PIRO order $n$ versus $B^{-1}$. The linear fit yields $s \simeq 3.4 \mathrm{~km} / \mathrm{s}$. The data were obtained at $T=3.5 \mathrm{~K}$ in a $100 \mu \mathrm{m}$ wide Hall bar sample with $n_{e} \simeq 3.8 \times 10^{11} \mathrm{~cm}^{-2}$ and $\mu \simeq 1.2 \times 10^{7} \mathrm{~cm}^{2} / \mathrm{V} \mathrm{s}$. Adapted from Hatke et al. (2009a).

characteristic frequency $\omega_{\pi}=2 k_{F} s$. Here $s$ is the speed of sound and the out-of-plane component of the phonon momentum is neglected. Such interaction causes a correction to the resistivity $\Delta \rho^{\mathrm{ph}}$ which oscillates with the ratio

$$
\epsilon_{\mathrm{ph}}=\omega_{\pi} / \omega_{\mathrm{c}} \propto n_{e}^{1 / 2}
$$

The $n_{e}^{1 / 2}$ dependence was confirmed experimentally (Zudov et al., 2001b).

Electron backscattering by an acoustic phonon is most effective when $\omega_{\pi}=n \omega_{\mathrm{c}}, n=1,2 \ldots$, which maximizes the (thermally averaged) product of the initial and final densities of states. In the simplest model of $2 \mathrm{D}$ isotropic phonons, the oscillatory part of the resistivity for $\epsilon_{\mathrm{ph}} \gtrsim 1$ and $T \gg \omega_{\mathrm{c}}, \omega_{\pi}$ reads (Dmitriev et al., 2010)

$$
\frac{\Delta \rho^{\mathrm{ph}}}{\rho_{\mathrm{D}}} \simeq \frac{2 g^{2} T \tau}{\pi \sqrt{\epsilon_{\mathrm{ph}}}} \delta^{2} \cos \left(2 \pi \epsilon_{\mathrm{ph}}-\pi / 4\right)
$$

[see also Eq. (161)]. The phase $-\pi / 4$ was confirmed experimentally by Hatke et al. $(2011 \mathrm{e})$ in a variety of high mobility samples. Many other experiments (Bvkov and Goran, 2009; Bvkov et al., 2005a; Hatke et al., 2009a; Yang et al., 2002b; Zhang et al., 2008b; Zudov et al., 2009, 2001b) reported PIRO maxima at integer $\epsilon_{\mathrm{ph}}$. As discussed in Sec.V.G the phase of PIRO (unlike that for MIRO and HIRO) is not expected to be universal and is sensitive to the anisotropy of relevant phonon modes, crystallographic orientation of the sample, width of the QW, etc. (Dmitriev et al., 2010; Raichev, 2009).

In contrast to other low- $B$ magnetoresistance oscillations ( $\mathrm{SdH}$ oscillations, MIRO, MISO, and HIRO), which are observed at low $T$, PIRO are best resolved at $T \sim \omega_{\pi}$ and get strongly suppressed at both low and high $T$ (Bvkov and Goran, 2009; Hatke et al., 2009a; Yang et al., 2002b; Zudov et al., 2001b). At low $T$, both emission and absorption of $2 k_{F}$-phonons is exponentially suppressed by the thermal factors for relevant electron 
and phonon states, see Sec.V.G. On the other handsimilar to MIRO, MISO, and HIRO-PIRO are insensitive to the temperature smearing of the Fermi surface which suppresses $\mathrm{SdH}$ oscillations at $2 \pi^{2} T / \omega_{\mathrm{c}} \gg$ 1. A detailed study of the $T$ dependence of PIRO revealed that higher- $B$ (lower- $\epsilon_{\mathrm{ph}}$ ) oscillations are best developed at progressively higher $T$ : the temperature at which a given oscillation reaches its maximum amplitude was found to scale with $\sqrt{B}$ (Bykov and Goran, 2009; Hatke et al., 2009a). This suggests that, at high $T$, the suppression of PIRO (similar to MIRO and HIRO, see Secs. III.A, III.B.1.f and V.A. but unlike the SdH oscillations) is due to interaction-induced broadening of LLs.

Similar to HIRO and MIRO, PIRO are most pronounced in high-quality 2DEGs. Early experiments in moderate mobility samples $\left(\mu \sim 10^{6} \mathrm{~cm}^{2} / \mathrm{Vs}\right)$ required temperatures in the range about $5-20 \mathrm{~K}$ and revealed only a few oscillations (Bykov et al., 2005a; Zudov et al., 2001b). Recent experiments in high mobility samples $\left(\mu \sim 10^{7} \mathrm{~cm}^{2} / \mathrm{Vs}\right)$ showed up to eight oscillations (Fig. 31) which remained visible down to $T \simeq 2 \mathrm{~K}$ (Hatke et al., 2009a).

Related phonon-induced oscillations in the magnetothermopower were observed by Zhang et al. (2004). Interplay of PIRO and MISO was investigated by Bykov et al. (2010a) in a single GaAs QW with two populated subbands.

\section{F. PIRO in a strong Hall field}
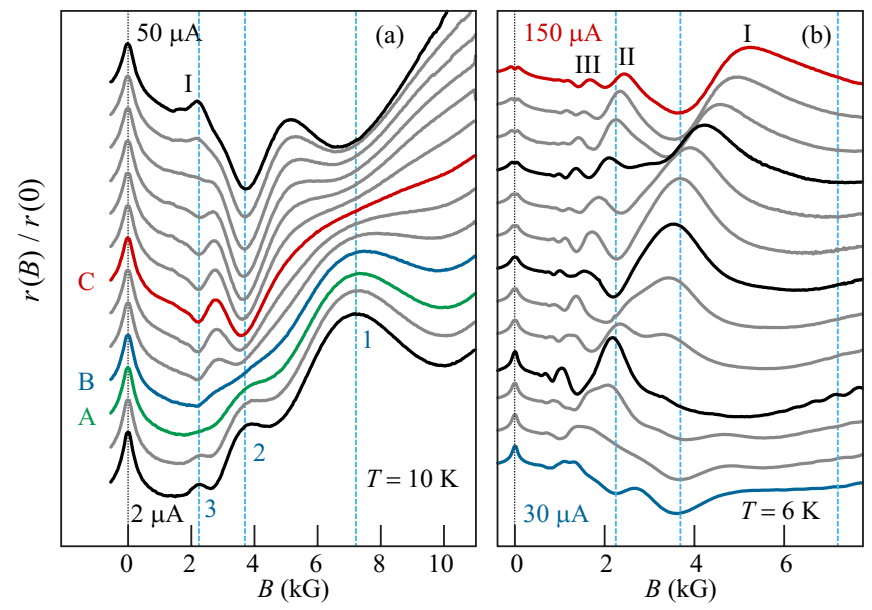

FIG. 32 Differential magnetoresistivity $r$ as a function of $B$, normalized to the resistivity at $B=0$, for different $I$ (a) from 2 to $50 \mu \mathrm{A}$ in steps of $4 \mu \mathrm{A}$ at $T=10 \mathrm{~K}$ and (b) from 30 to $150 \mu \mathrm{A}$ in steps of $10 \mu \mathrm{A}$ at $T=6 \mathrm{~K}$. The traces are offset for clarity. Integers I, II, and III at the top traces mark the order of the HIRO peaks. The vertical lines correspond to integer $\epsilon_{\mathrm{ph}}=1,2$ and 3 . The curves labeled by (A), (B), and $(\mathrm{C})$ in (a) are discussed in the caption to Fig. 33. The data were obtained in a $50 \mu \mathrm{m}$ wide Hall bar sample with $n_{e} \simeq 4.8 \times 10^{11} \mathrm{~cm}^{-2}$ and $\mu \simeq 4.4 \times 10^{6} \mathrm{~cm}^{2} / \mathrm{V} \mathrm{s}$. Adapted from Zhang et al. (2008b).
Recent studies (Zhang et al., 2008b) of PIRO in a strong Hall field established that the dc field leads to the evolution of the PIRO maxima into minima and back, see Fig.32. It was also found that the strong dc field enables detection of PIRO at low $T$ where the linear-response PIRO are exponentially suppressed. One additional experimental finding was the observation of a pronounced resistance maximum at $\epsilon_{\mathrm{dc}}=\epsilon_{\mathrm{ph}}$, where the Hall velocity equals the speed of sound. All these results are reproduced within the theoretical model of Dmitriev et al. (2010), see Sec.V.G.

\section{G. Microscopic theory of PIRO}

In the case of MIRO (Sec. III.B) or nonlinear dc transport (Sec. V.B), the inelastic effects that lead to oscillations in the energy distribution of electrons play an essential and often dominant role. By contrast, PIRO are observed at elevated $T$, where a fast inelastic relaxation makes effects of this type much less important. Therefore, one can consider only effects that are similar to the displacement contribution to HIRO and MIRO. In terms of migration of the guiding centers of cyclotron orbits [Eqs. (65)-(67)], the phonon-assisted dissipative current $j_{d}^{(p)}$ has the form of Eq. (65) with

$$
\begin{aligned}
& W_{x_{1} \rightarrow x_{2}}=\left\langle\int d \varepsilon_{1} \int d \varepsilon_{2} \mathcal{M}_{\varepsilon_{1} \varepsilon_{2}} \delta\left(x_{1}-x_{2}+\Delta X_{\varphi_{1} \varphi_{2}}\right)\right. \\
& \left.\times \sum_{ \pm} \Gamma_{\varphi_{1} \varphi_{2}}^{(\mathrm{sp})}\left(\mathcal{N}_{\omega_{12}}+\delta_{1, \pm 1}\right) \delta\left(\Delta \varepsilon_{12}^{\mathrm{tot}} \mp \omega_{12}\right)\right\rangle_{\varphi_{1} \varphi_{2}}
\end{aligned}
$$

where $\Gamma_{\varphi_{1} \varphi_{2}}^{(\mathrm{sp})}$ denotes the probability of spontaneous emission of a phonon with frequency $\omega_{12}$ and $\mathcal{N}_{\omega}$ is the Planck distribution function. In the factor $\mathcal{M}_{\varepsilon \varepsilon^{\prime}}$ [Eq. [67)], one can substitute, in accordance with the above, $f_{\varepsilon}^{T}$ for $f_{\varepsilon}$. Similar to Eq. (66), the delta function containing $\Delta \varepsilon_{12}^{\text {tot }}$ ensures energy conservation.

We start with a simplified single-mode model which assumes interaction with 2D isotropic acoustic phonons via a deformation potential. We also assume that the out-ofplane component of the phonon momentum is negligible, i.e., the width $b$ of the QW to which the 2DEG is confined is large, $b \gg k_{F}^{-1}$. In this case,

$$
\Gamma_{\varphi_{1} \varphi_{2}}^{(\mathrm{sp})}=g^{2} \omega_{12} / 2, \quad g^{2}=m \mathcal{D}^{2} / \rho b s^{2},
$$

where phonon frequency for quasielastic scattering is given by $\omega_{12}=2 k_{F} s \sin \left|\left(\varphi_{1}-\varphi_{2}\right) / 2\right|, \rho$ is the mass density, and $\mathcal{D}$ is the deformation-potential constant. In overlapping LLs and at high $T \gg \omega_{\mathrm{c}}, \omega_{\pi}$, Eqs. (65), (159), and (160) yield (Dmitriev et al., 2010)

$$
j_{d}^{(p)}=\sigma_{\mathrm{D}} E \tau g^{2} T\left(1+2 \delta^{2}\left[J_{0}\left(2 \pi \epsilon_{\mathrm{ph}}\right)-J_{2}\left(2 \pi \epsilon_{\mathrm{ph}}\right)\right]\right) .
$$

The linear-in- $T$ dependence of $j_{d}^{(p)}$ is due to the fact that at $T \gg \omega_{\pi}$ the occupation number for relevant phonon 
modes is large, so that $\Gamma_{\varphi_{1} \varphi_{2}}^{(\mathrm{sp})} \mathcal{N}_{\omega_{12}} \simeq g^{2} T / 2$, while the contribution of spontaneous emission is negligible. The current shows oscillations with $\epsilon_{\mathrm{ph}}=\omega_{\pi} / \omega_{\mathrm{c}}$, controlled by commensurability between the cyclotron energy and the maximum possible - in the process of scatteringphonon energy. That is, in accord with the original interpretation (Zudov et al., 2001b), PIRO originate from resonant inter-LL transitions caused by the backscattering of electrons by acoustic phonons. The position of higher-order maxima of PIRO in Eq. (161) at $\epsilon_{\mathrm{ph}} \gg 1$ is given by Eq. (158). In narrow QWs $\left(k_{F} b \sim 1\right)$ or in wide QWs for $\epsilon_{\mathrm{ph}} \gg\left(k_{F} b\right)^{2}$, one should take into account the $3 \mathrm{D}$ character of acoustic phonons. Still in the isotropic approximation, the $\cos \left(2 \pi \epsilon_{\mathrm{ph}}-\pi / 4\right)$ behavior of PIRO [Eq. (158)] changes then to $\cos \left(2 \pi \epsilon_{\mathrm{ph}}\right)$, while the amplitude of PIRO reduces by a factor of $\pi \epsilon_{\mathrm{ph}}^{1 / 2} / 2 b k_{F}$ (Raichev, 2009).

While the isotropic single-branch model captures the essential physics of PIRO, in real structures the electronphonon interaction is more complicated. In bulk GaAs, there are three anisotropic phonon branches and two mechanisms (via the deformation and piezoelectric potentials) of electron-phonon interaction. A comprehensive study of PIRO, using the general form of interaction with bulk acoustic phonons, was performed by Raichev (2009). For higher harmonics of PIRO, the effects of anisotropy were treated there analytically, producing three distinct contributions with different phases and periods (Raichev, 2009). Additional contributions can arise from interaction with interface phonon modes (Zudov et al., 2001b).

The analysis by Raichev (2009) shows that PIRO in the ultra-high mobility and moderate electron density sample used by Hatke et al. (2009a) are dominated by the bulk transverse acoustic mode propagating along the $2 \mathrm{D}$ plane and polarized perpendicular to the plane. The sound velocity of this mode $s_{T O}=3.40 \mathrm{~km} / \mathrm{s}$ is indeed very close to $s=3.44 \mathrm{~km} / \mathrm{s}$ extracted from the period of PIRO by (Hatke et al., 2009a). A subleading contribution of the longitudinal mode with a noticeably higher velocity produces an extra peak observed by Hatke et al. (2009a) at sufficiently high $B$, and also explains the beating pattern at lower $B$, caused by the interference of these two contributions to PIRO [see Fig. 1 in (Raichev, 2009)]. By contrast, in the moderate mobility and high electron density sample studied by (Bykov et al., 2005a), PIRO are dominated by the higher-energy longitudinal mode. In another recent experiment (Zhang et al., 2008b), which studied the effects of a strong dc field on PIRO (discussed below), the longitudinal mode also prevailed, which explains the good agreement between experiment (Zhang et al., 2008b) and theory (Dmitriev et al., 2010) based on the single-mode approximation (160).

The Hall field tilts LLs and changes the commensurability condition for electron-phonon scattering. This results in oscillations with $\epsilon_{ \pm} \equiv \epsilon_{\mathrm{dc}} \pm \epsilon_{\mathrm{ph}}$, where $\epsilon_{\mathrm{dc}}$ is the parameter that controls HIRO (Sec. V.B). The change of the oscillations period by the dc field can be

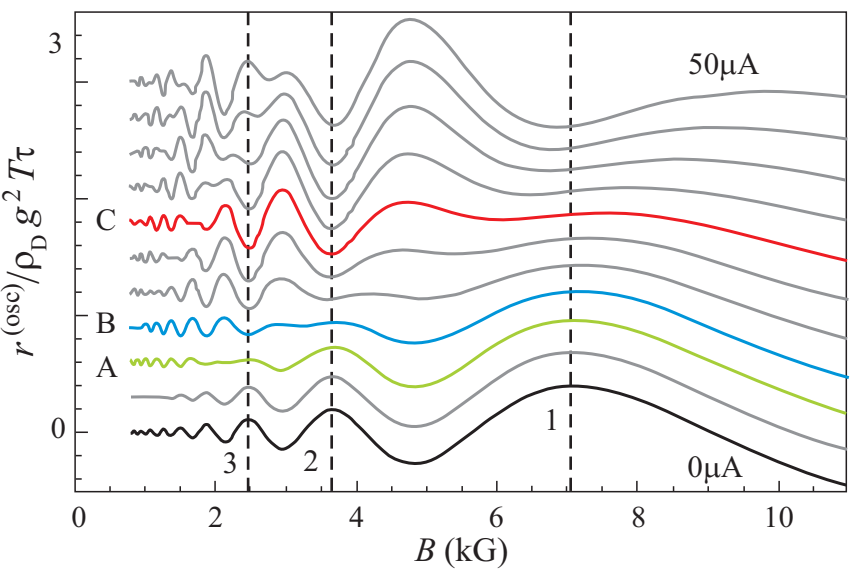

FIG. 33 Calculated oscillatory part $r^{\text {(osc) }}$ of the differential resistivity $\partial E / \partial j_{d}$ versus $B$, in units of $\rho_{\mathrm{D}} g^{2} T \tau$, for several values of current $I$ from 0 to $50 \mu \mathrm{A}$ in steps of $5 \mu \mathrm{A}$. The traces are offset for clarity. The vertical lines correspond to integer $\epsilon_{\mathrm{ph}}$. The traces for (A) $10 \mu \mathrm{A}$, (B) $15 \mu \mathrm{A}$, and (C) $30 \mu \mathrm{A}$ correspond approximately to the values of $I$ at which the third, second, and first PIRO peaks, respectively, disappear before evolving into minima as $I$ increases. The curves are very similar to those measured in the experiment: cf. the curves in Fig. 32 labeled as A $(10 \mu \mathrm{A}), \mathrm{B}(14 \mu \mathrm{A})$, and $\mathrm{C}$ $(28 \mu \mathrm{A})$. From Dmitriev et al. (2010).

viewed as a Doppler shift of the phonon modes in the frame moving with the Hall velocity $v_{H}=c E / B$ across the field. In the moving frame, the electric field is absent while the phonon dispersion becomes anisotropic, $s \rightarrow s-v_{H} \cos \left[\left(\varphi_{1}+\varphi_{2}\right) / 2\right]$ (this formulation is particularly useful in the case of a complicated dispersion relation of phonons). For $1 \ll \epsilon_{\mathrm{dc}}<\epsilon_{\mathrm{ph}}$ and $1 \ll \epsilon_{\mathrm{ph}}-\epsilon_{\mathrm{dc}}$, Eqs. (65), (159), and (160) yield the following for the oscillating part of $j_{d}^{(\mathrm{p})}$ :

$$
\frac{j_{d}^{(\mathrm{p}, \mathrm{osc})}}{\sigma_{\mathrm{D}} E}=\frac{4 \delta^{2}}{\pi^{2}} g^{2} T \tau\left(\frac{\sin 2 \pi \epsilon_{+}}{\sqrt{\epsilon_{\mathrm{dc}} \epsilon_{+}}}+\frac{\cos 2 \pi \epsilon_{-}}{\sqrt{\epsilon_{\mathrm{dc}}\left|\epsilon_{-}\right|}}\right) .
$$

The evolution of the differential resistivity $r=\partial E / \partial j_{d}$ with varying $\epsilon_{\mathrm{dc}}$ in this regime was studied experimentally by Zhang et al. (2008b). The results of calculation according to Eqs. (159) and (160), illustrated in Fig. 33. quantitatively reproduce the experimental data in Fig. 32 without fitting parameters. Note that the theoretical plot does not include HIRO which become relevant at higher dc bias, see Fig.32b, but are negligible in the region of interest, $B>2 \mathrm{kG}$ and $I<40 \mu \mathrm{A}$. In qualitative agreement with the experimental results of Zhang et al. (2008b), similar oscillations with $\epsilon_{\mathrm{ph}}-\epsilon_{\mathrm{dc}}$ were also obtained numerically, using the balance-equation approach, by Lei (2008).

In a stronger $\mathrm{dc}$ field, $\epsilon_{\mathrm{dc}}>\epsilon_{\mathrm{ph}}$, corresponding to a supersonic Hall velocity $v_{H}>s$, the theory of Dmitriev et al. (2010) predicts that the phase of the oscillations changes in the second term of Eq. (162) from $\cos 2 \pi \epsilon_{-}$to $\sin 2 \pi \epsilon_{-}$for $\epsilon_{\mathrm{dc}}>\epsilon_{\mathrm{ph}}$. Note that at $v_{H}>s$, 
the effective sound velocity $s-v_{H} \cos \left[\left(\varphi_{1}+\varphi_{2}\right) / 2\right]$ in the moving frame becomes negative for certain transitions. Emission of a phonon with energy $\omega$ in such transitions increases the electron kinetic energy by $\Delta>0$ at the expense of the electrostatic energy which is decreased by $\Delta+\omega$.

The physical meaning of the "sound barrier" is clear from the low-temperature behavior of the phononassisted transport. At $T \ll \omega_{\pi}$, stimulated emission and absorption of phonons are exponentially suppressed by the Planck factor $\mathcal{N}_{\omega_{\pi}} \simeq \exp \left(-\omega_{\pi} / T\right)$ in Eq. (159). On the other hand, spontaneous emission in the scattering processes reducing the kinetic energy is also strongly suppressed by the factor $\mathcal{M}_{\varepsilon_{1} \varepsilon_{2}}$. However, in the spontaneous emission processes that increase the kinetic energy, the constraint imposed by the factor $\mathcal{M}_{\varepsilon_{1} \varepsilon_{2}}$ is not effective. As a result, at $\epsilon_{\mathrm{dc}}>\epsilon_{\mathrm{ph}}$ the phonon-assisted transport due to spontaneous phonon emission survives even at $T=0$. Specifically, for $1 \ll \epsilon_{-} \ll \epsilon_{\mathrm{dc}}$ the phononinduced oscillations at $T \ll \omega_{\pi}$ are described by

$$
\frac{j_{d}^{(\mathrm{p}, \mathrm{osc})}}{\sigma_{\mathrm{D}} E \tau}=\frac{2 \delta^{2} \epsilon_{\mathrm{ph}} \epsilon_{-}^{1 / 2}}{\pi^{2} \epsilon_{\mathrm{dc}}^{3 / 2}} g^{2} \omega_{\mathrm{c}} \sin 2 \pi \epsilon_{-} .
$$

The evolution of the oscillations with temperature is illustrated in Fig. 34 (where also the smooth part of the phonon-assisted current at $T=0$ is shown).

Nonlinear mixing of PIRO and MIRO was investigated by Raichev (2010b). This study predicted oscillations with $\omega_{\pi} \pm \omega$ and discussed the feasibility of their experimental observation. Further, the analysis by Raichev $(2010 \mathrm{~b})$ showed that heating of the 2DEG leads to additional $\omega_{\pi} / \omega_{\mathrm{c}}$-oscillations with a phase shift with respect to the equilibrium PIRO. Separately, nonlinear mixing of PIRO and MISO in two-subband systems was shown by Bykov et al. (2010a); Raichev (2010a) to lead to oscillations at frequencies determined by $\omega_{\pi}$ and the subband splitting energy. A further example of frequency mixing for the case of MIRO and HIRO is discussed in Sec.VI.B.2

\section{TRANSPORT IN STRONG AC AND DC FIELDS}

In the preceding sections, we discussed two types of nonequilibrium magnetooscillations, MIRO (Sec.III) and HIRO (Sec.V). The nature of MIRO has so far been considered at the level of single-photon processes. We now turn to mode-mixing phenomena; specifically, to multiphoton processes in MIRO and to nonlinear mixing of MIRO and HIRO.

\section{A. Fractional MIRO}

\section{Experiments at high microwave power levels}

In addition to MIRO, whose extrema in the vicinity of integer $\epsilon_{\mathrm{ac}}$ values are described by Eq. (63) and the
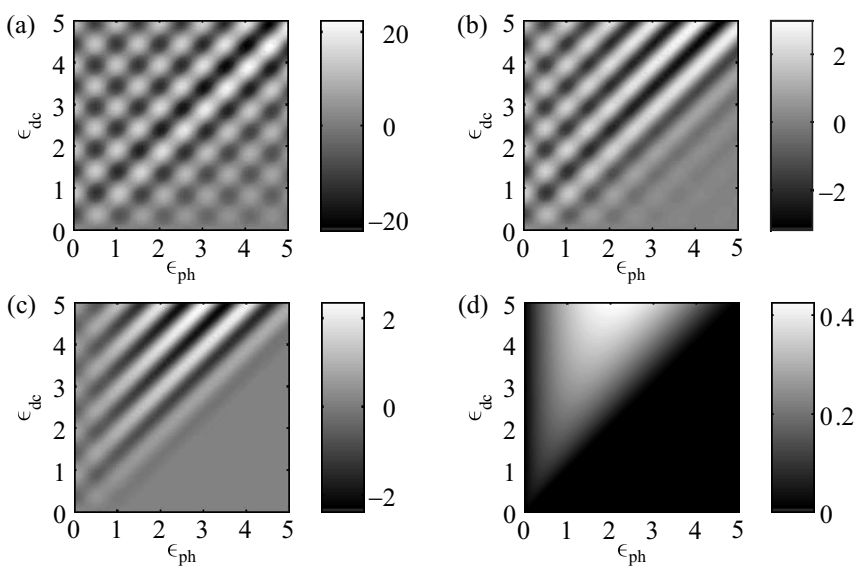

FIG. 34 (a)-(c) Oscillatory part of the phonon-assisted current $j_{d}^{\text {(p,osc) }}$ in units of $2 \delta^{2} g^{2} \omega_{\mathrm{c}} \tau \sigma_{\mathrm{D}} E$, calculated for (a) $T=$ $5 \omega_{\mathrm{c}}$, (b) $T=0.7 \omega_{\mathrm{c}}$, and (c) $T=0.25 \omega_{\mathrm{c}}$. (d) Smooth part of the phonon-assisted current at $T=0$. From Dmitriev et al. (2010).

oscillatory structure is shown in Fig. 6, similar oscillatory features have been observed near $\epsilon_{\mathrm{ac}}$ given by certain rational fractions, with maxima $(+)$ and minima $(-)$ of the photoresistance at

$$
\epsilon_{\mathrm{ac}}^{ \pm}=\frac{n}{m} \mp \varphi_{\mathrm{ac}}^{ \pm}
$$

where $n$ and $m$ are integers (with noninteger $n / m$ ) and $\varphi_{\mathrm{ac}}^{ \pm}>0$ is, in typical experiments, considerably smaller than $1 / 4$ (Fig. [35). The oscillatory feature at $\epsilon_{\mathrm{ac}}=$ $1 / 2$ was observed by Dorozhkin (2003); Dorozhkin et al. (2007b, 2005); Wiedmann et al. (2009a); Willett et al. (2004) [in retrospective, the first signature of the halfinteger MIRO can be recognized already in early experiments on moderate-mobility samples (Zudov et al., 2001a), see a weak oscillation at $B \simeq 2 \mathrm{kG}$ in the $f=45 \mathrm{GHz}$ trace there]. Similar fractional MIRO (fMIRO) for other fractions in the $n=1$ series $\epsilon_{\mathrm{ac}}=$ $1 / m$ were reported for $m=3,4$ (Dorozhkin et al., 2007b; Wiedmann et al., 2009a) and $m=5,6,7,8$ (Wiedmann et al., 2009a). Fractions that have been observed in the fMIRO series with $n=2$ (apart from the even denominator fractions coinciding with the members of the $n=1$ series) are represented by $m=3$ (Dorozhkin et al., 2007b; Wiedmann et al., 2009a; Zudov, 2004; Zudov et al., 2006a) and $m=5,7$ (Wiedmann et al., 2009a). Note that the fMIRO series with $n=1,2$ belong to the high- $B$ side of the $\mathrm{CR}$ and thus do not overlap with the integer MIRO which occur at $\epsilon_{\mathrm{ac}}>1$. A third series of fMIRO among those observed is, in contrast to the variable-denominator series above, characterized by fixed $m=2$ and variable $n$. The reported oscillatory features in the $\epsilon_{\mathrm{ac}}=n / 2$ series that fall in the intervals between integer $\epsilon_{\mathrm{ac}}$ are (apart from $\epsilon_{\mathrm{ac}}=1 / 2$ mentioned above) those with $n=3$ and 5 (Zudov, 2004; Zudov et al., 2003, 2006a). The halfinteger fMIRO with $n \geq 3$ lie on the low- $B$ side of the 

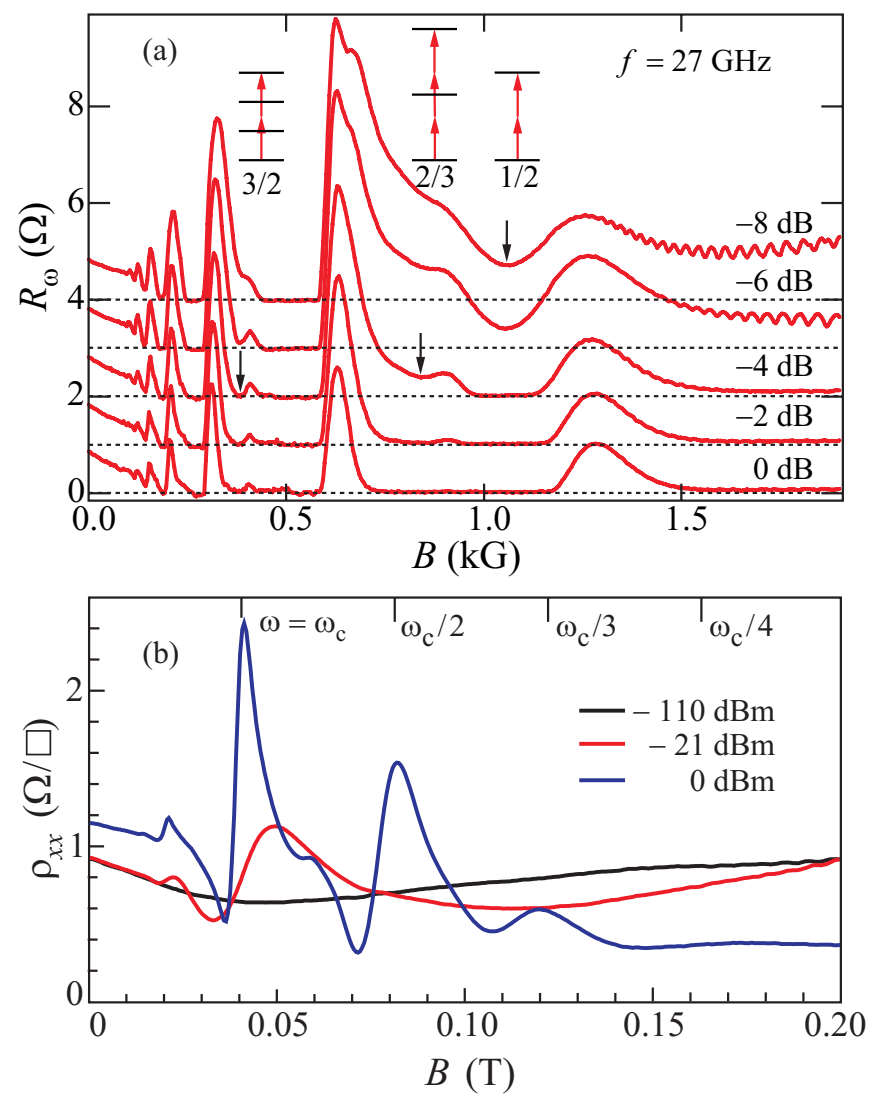

FIG. 35 (a) Magnetoresistance under microwave illumination at $f=27 \mathrm{GHz}$ for different radiation intensities. The traces, offset vertically for clarity, are labeled according to the attenuation levels. The downward arrows mark the minima near $\epsilon_{\mathrm{ac}}=3 / 2,2 / 3$, and $1 / 2$, which develop into fractional ZRS at higher radiation intensities. The inset illustrates multiphoton processes responsible for these features. The data were obtained at $T \simeq 1.0 \mathrm{~K}$ in a sample with $n_{e} \simeq 3.6 \times 10^{11} \mathrm{~cm}^{-2}$ and $\mu \simeq 2 \times 10^{7} \mathrm{~cm}^{2} / \mathrm{V}$ s. From Zudov et al. (2006b). (b) Similar data for $f=17 \mathrm{GHz}$ at $T \simeq 1.4 \mathrm{~K}$ in a sample with $n_{e} \simeq 2.7 \times 10^{11} \mathrm{~cm}^{-2}$ and $\mu \simeq 1.7 \times 10^{7} \mathrm{~cm}^{2} / \mathrm{Vs}$. From Dorozhkin et al. (2007b).

$\mathrm{CR}$ and therefore require for their observation (other experimental conditions being equal) higher mobilities compared to the fMIRO series with $n=1$.

Similar to MIRO, the most prominent minima from all three fMIRO series, namely those in the vicinity of $\epsilon_{\mathrm{ac}}=1 / 2,2 / 3,3 / 2$, were shown to evolve into ZRS with increasing microwave power in ultra-high mobility samples (Zudov et al., 2006a). As seen in Fig. 35a , the formation of these fractional ZRS was accompanied by the diminishing and narrowing of the neighboring photoresistance peaks, as well as by the overall suppression of the photoresistance at $\epsilon_{\mathrm{ac}}<1 / 2$. A similar strong suppression of the resistance by radiation on the high- $B$ side of the CR was examined by Dorozhkin et al. (2005).

\section{Multiphoton absorption via virtual states}

The theoretical framework developed to describe fMIRO relates them to multiphoton processes in which $m$ photons are absorbed in transitions between electron states separated in energy by $n \omega_{c}$ (Dmitriev et al., 2007b; Lei and Liu, 2003, 2006b; Pechenezhskii et al., 2007; Torres and Kunold, 2005), as illustrated in the inset to Fig. 35h. One can distinguish two (generally, interfering with each other) scattering channels for multiphoton transitions: sequential absorption of single photons via real (resonant) intermediate electron states and multiphoton absorption via virtual intermediate states. For overlapping LLs, both types of multiphoton transitions yield in the limit of a strong ac field oscillatory features in the vicinity of $\epsilon_{\mathrm{ac}}=n / m$-with an amplitude proportional at $T \gg m \omega$ to $\delta^{2 m}$ - which, for given $\delta$, are damped by disorder much more strongly than the integer MIRO (Pechenezhskii et al., 2007). For separated LLs, however, single-photon real transitions are forbidden if $\omega$ is not sufficiently large to bridge the gap between the LLs (see Fig. 12), so that microwave absorption occurs then by means of multiphoton transitions via virtual states. This is, in particular, the case for two-photon transitions between neighboring LLs at $\omega=\omega_{c} / 2 \gg 1 / \tau_{\mathrm{q}}$.

In the limit of well-separated LLs, the mechanism of multiphoton absorption via virtual intermediate states is much similar to that of multiphoton ionization in atomic or semiconductor systems (Keldvsh, 1965). In particular, a perturbative expansion in the radiation power is justified if $\omega$ is large compared to the transition rate between LLs the slope of which oscillates in phase with the ac field. The parameter that governs the validity of perturbation theory is $\left(\tau_{\mathrm{a}} / \tau\right) \mathcal{P} \ll 1$, where $\mathcal{P}$ is given by Eq. (72) (Dmitriev et al., 2007a). Here and in the rest of Sec. VI.A we overview the approach developed by Dmitriev et al. (2007b) and Pechenezhskii et al. (2007) to describe fMIRO for the case of smooth disorder. We expect that the picture that results from this approach remains qualitatively correct for more general models of disorder. Similar to the integer MIRO, fMIRO induced by the inelastic mechanism (Dmitriev et al., 2007b) are much stronger than those induced by the displacement mechanism $^{19}$ (Dmitriev et al., 2007b; Lei and Liu, 2003, 2006b; Torres and Kunold, 2005), provided $\tau_{\text {in }} / \tau_{\mathrm{q}} \gg 1$. Under this condition, the half-integer fMIRO near $\epsilon_{\mathrm{ac}}=$

19 The method of "force- and energy-balance equations" Lei, 2010; Lei and Liu, 2003, 2006b) tacitly assumes an instant thermal equilibration in the frame moving with the drift velocity, i.e., the inelastic mechanism of photoconductivity is lost within this formalism "by construction". Similarly, the argument by Torres and Kunold (2005) that the "inelastic processes can be safely ignored" if they are slower than the elastic ones is in contradiction with the fact that without them the stationary regime of photoconductivity cannot be established: in effect, Torres and Kunold (2005) also implicitly assume an infinitely fast equilibration of the same kind. 
$n / 2$ with $n=1,3,5, \ldots$ are described at order $\mathcal{P}^{2}$ for well-separated LLs (Dmitriev et al., 2007b) by

$$
\frac{\rho_{\mathrm{ph}}}{\rho_{\mathrm{D}}}=\frac{3 \tau_{\mathrm{in}} \tau_{\mathrm{q}}}{32 \tau^{2}} \mathcal{P}^{2} \mathcal{R}_{2}(2 \omega)(1+\vartheta),
$$

where the function $\mathcal{R}_{2}(\omega)$, odd in the detuning $2 \omega-n \omega_{c}$, is defined in Eq. (85), and $\vartheta=2\left|\mathcal{E}_{+} \mathcal{E}_{-}\right|^{2} / \mathcal{P}^{2}$ depends on the polarization of microwaves; specifically, $\vartheta=\left[\left(\omega^{2}-\right.\right.$ $\left.\left.\omega_{c}^{2}\right) /\left(\omega^{2}+\omega_{c}^{2}\right)\right]^{2} / 2$ and $\vartheta=0$ for the cases of linear and circular polarization, respectively. For $n=1$, Eq. (165) describes the two-photon $\mathrm{CR}$ in the photoresistivity.

The two-photon contribution of the displacement mechanism is smaller (in the parameter $\tau_{\mathrm{q}} / \tau_{\text {in }} \ll 1$ ) but possesses, in the case of linear polarization, anisotropy with respect to the mutual orientation of the dc and ac fields. At order $\mathcal{P}^{2}$, the depth of the modulation, as the orientation is changed, between the principal values of the photoresistivity tensor induced by the displacement mechanism is given by the factor $1 \pm \sqrt{2 \vartheta} /(1+\vartheta)$ (for the full analytical expression, see Dmitriev et al., 2007b). The orientation dependence thus provides a measure of the role of the displacement mechanism in fMIRO.

\section{Multiphoton absorption via sidebands}

The multiphoton absorption via virtual states [Eq. (165)] is the main mechanism of fMIRO in the limit of well-separated LLs. A subleading (in the parameter $\left.1 / \omega_{c} \tau_{\mathrm{q}} \ll 1\right)$ contribution to fMIRO comes from single-photon transitions via radiation-induced "sidebands" formed in the gaps of the DOS between the LLs (Dmitriev et al., 2007b). The sideband mechanism can be viewed as resulting from disorder-induced transitions between the quasienergy levels (Zeldovich, 1967) that characterize the stationary states of a homogeneous electron system under the action of a periodicin-time perturbation. A systematic approach to studying the spectral properties of a 2DEG driven by the ac field in the presence of disorder is formalized in terms of a time-dependent self-energy of the Wignertransformed retarded Green's function (Dmitriev et al., 2007a,b; Vavilov and Aleiner, 2004). The notion of a time-dependent DOS was also introduced to describe sidebands in a closely related problem of the dynamical Franz-Keldysh effect (Yacobv, 1968) by Jauho and Johnsen (1996); Johnsen and Jauho (1998). In the fMIRO problem, the nonequilibrium DOS of each LL acquires, to first order in $1 / \omega_{c} \tau_{\mathrm{q}}$, two static satellite peaks

$$
\nu_{ \pm}^{\mathrm{sb}}(\epsilon)=\left(\pi \mathcal{P} / 8 \omega_{c} \tau\right) \nu^{(N)}(\epsilon \pm \omega),
$$

where $\nu^{(N)}(\epsilon)$ is given by Eq. (27), centered for $\omega=\omega_{c} / 2$ in the middle between LLs, and also satellites centered at the same energies and oscillating in time with a pe$\operatorname{riod} 2 \pi / \omega$ (Dmitriev et al., $2007 \mathrm{~b})$. Note that the DOS peaks (166) can be probed by resonant spectroscopy in the same way as if they were present at equilibrium. For the half-integer fMIRO, single-photon transitions to and from the static sidebands yield a contribution which, at order $\mathcal{P}^{2}$, has the same shape as in Eq. (165) and is of order $\omega_{c} \tau_{\mathrm{q}}$ times smaller (Dmitriev et al., 2007b). On the other hand, the time-dependent sidebands give a contribution which is smaller than that in Eq. (165) by only a factor of order $\left(\omega_{c} \tau_{\mathrm{q}}\right)^{1 / 2}$ and has a distinctly different shape; in particular, it is even in the detuning from the fMIRO resonance (for details, see Dmitriev et al., $2007 \mathrm{~b}$ ).

\section{Multiphoton absorption via real states}

Multiphoton absorption via virtual states or sidebands provides the mechanisms of fMIRO in the case of wellseparated LLs. If, however, gaps between LLs are opened (i.e., $\omega_{c} \tau_{\mathrm{q}}>\pi / 2$, Sec. II.C.1), but the width of the gaps $\Delta_{g}<\omega_{c} / 2$, then for any given $\omega$ in the interval $\Delta_{g}<\omega<\omega_{c}-\Delta_{g}$ real single-photon transitions are possible both between neighboring LLs and within the same LL. For $\omega$ from the above interval, an electron can thus be transferred between neighboring LLs via two sequential single-photon transitions. More generally, for $(n-1) \omega_{c}+\Delta_{g}<\omega<n \omega_{c}-\Delta_{g}$, single-photon real transitions are possible with a change of the LL index by both $n-1$ and $n$, where $n=1,2,3, \ldots$ For given $n$, therefore, there exists a finite range of $\omega$ and $\omega_{c}$ within which $m \neq n$ photons can bridge the gap between $n$ LLs via real intermediate states, which leads to the emergence of an additional mechanism of fMIRO (Dorozhkin et al., 2007b; Pechenezhskii et al., 2007). fMIRO induced by this mechanism of sequential single-photon absorption are specific to the crossover between the regimes of overlapping and well-separated LLs and disappear altogether for $2 \Delta_{g}>\omega_{c}$. At the crossover, however, they are the main fMIRO channel at order $\mathcal{P}^{2}$ if $\tau_{\text {in }} / \tau_{\mathrm{q}} \gg 1$, with an amplitude proportional to $\left(\tau_{\text {in }} \mathcal{P} / \tau\right)^{2}$, similar to the second-order expansion in $\mathcal{P}$ of the integer MIRO contribution in Eq. (97).

\section{B. Nonlinear mixing of MIRO and HIRO}

The physics of high LLs out of equilibrium is further enriched by the possibility of simultaneously applying both ac and strong dc fields to make MIRO and HIRO superimpose onto each other. A nonlinear response of the 2DEG to the fields provides a way of mixing the "frequencies" $\epsilon_{\mathrm{ac}}$ and $\epsilon_{\mathrm{dc}}$ of the two types of magnetooscillations.

\section{Experiments on MIRO in a strong dc field}

The nonlinear mixing of MIRO and HIRO was demonstrated in experiments on ultra-high mobility structures by Zhang et al. (2007c) and Hatke et al. (2008b). Figure 36a shows the effect of a direct current $I$ varied un- 
der continuous microwave irradiation on the differential resistivity $r_{\omega}$ : the MIRO maxima are seen to evolve into minima, and vice versa, with increasing $I$. One of the most remarkable features in Fig. 36 is the crossing points (marked by arrows) of the curves measured at different currents: the resistivity shows little change at $\epsilon_{\mathrm{ac}}=n$ and $n+1 / 2$, where $n$ is an integer.

More insight into this phenomenon is gained if one plots $r_{\omega}$ as a function of $\epsilon_{\mathrm{dc}}$ for different fixed $\epsilon_{\mathrm{ac}}$, as in Fig. 36 $\mathrm{b}$. The amplitude of the oscillations of $r_{\omega}$ in Fig. 36b is seen to be much larger around $\epsilon_{\mathrm{ac}}=n \pm 1 / 4$ (near which $\epsilon_{\mathrm{ac}}$ the extrema of MIRO are located) than around $\epsilon_{\mathrm{ac}}=n, n+1 / 2$ (near which $\epsilon_{\mathrm{ac}}$ MIRO exhibit zero response). Figure $36 \mathrm{~b}$ also reveals a substantial difference between integer and half-integer $\epsilon_{\mathrm{ac}}$ : while at $\epsilon_{\mathrm{ac}}=2,3$ the oscillations are suppressed but still clearly visible, they virtually disappear at $\epsilon_{\mathrm{ac}}=5 / 2,7 / 2$. The phase of the oscillations of $r_{\omega}$ as a function of $\epsilon_{\mathrm{dc}}$ also shows a strong dependence on $\epsilon_{\mathrm{ac}}$. Specifically, at integer $\epsilon_{\mathrm{ac}}=2,3, r_{\omega}$ behaves very similar to HIRO, with maxima occurring at $\epsilon_{\mathrm{dc}} \simeq n$, Eq. (142). In contrast, the oscillations of $r_{\omega}$ at the MIRO maximum for $\epsilon_{\mathrm{ac}} \simeq 3-1 / 4$ and the MIRO minimum for $\epsilon_{\mathrm{ac}} \simeq 3+1 / 4$ are phase shifted by about a quarter cycle in opposite directions compared to HIRO.

As discussed in Sec.V.D, a minimum of HIRO may evolve into a ZdRS (Hatke et al., 2010). The nonlinear mixing of MIRO and HIRO gives rise to an interplay between ZRS associated with MIRO and ZdRS. Namely, as $\epsilon_{\mathrm{dc}}$ increases, ZRS that are present at zero dc field trans-
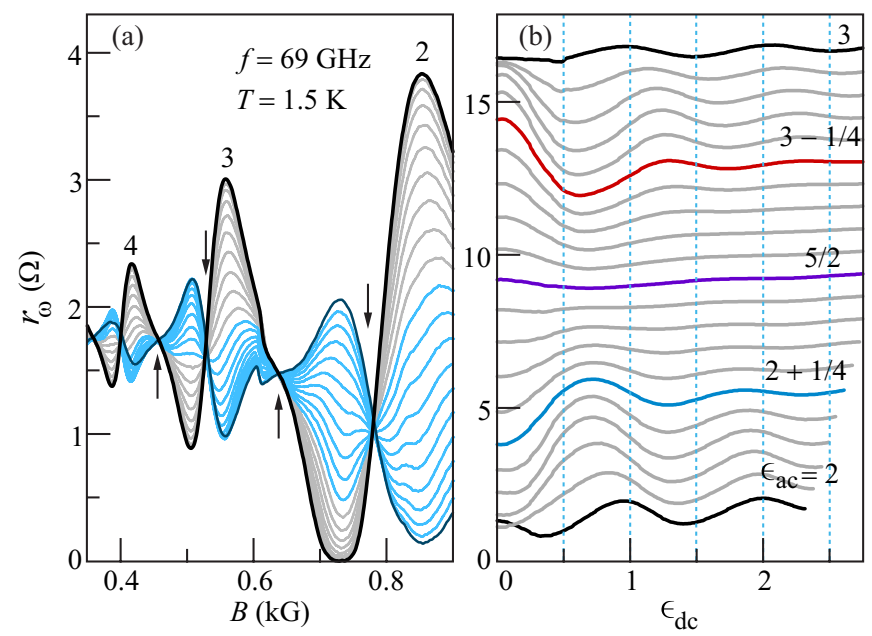

FIG. 36 (a) Differential resistivity $r_{\omega}$ versus magnetic field at a radiation frequency of $69 \mathrm{GHz}$ for different levels of dc excitation, from $I=0$ (thick black curve) to $20 \mu \mathrm{A}$ in steps of $1 \mu \mathrm{A}$. Integers show the order of the MIRO peaks. The arrows mark the zero-response nodes that remain largely immune to dc excitation. (b) Part of the data in (a), represented as the dependence of $r_{\omega}$ on $\epsilon_{\mathrm{dc}}$ at different fixed $\epsilon_{\mathrm{ac}}$ ranging from 2 to 3 in steps of 0.05 . The traces are offset in steps of $0.75 \Omega$. The data were obtained at $T=1.5 \mathrm{~K}$ in a $100 \mu \mathrm{m}$ wide Hall bar sample with $n_{e} \simeq 3.7 \times 10^{11} \mathrm{~cm}^{-2}$ and $\mu \simeq 1.2 \times 10^{7} \mathrm{~cm}^{2} / \mathrm{V} \mathrm{s}$. Adapted from Hatke et al. (2008b). form into maxima of $r_{\omega}$, as shown in Fig. 36a for the case of ZRS near $\epsilon_{\mathrm{ac}}=2+1 / 4$. In turn, a "strong maximum" of MIRO may be converted by the strong dc field into a ZdRS. The ZdRS in a microwave-driven $2 \mathrm{DEG}$, originating from the maximum of MIRO near $\epsilon_{\mathrm{ac}}=2-1 / 4$, was observed by Zhang et al. (2007c, 2008a). In fact, this observation appears to be an example of a more general (not relying on the presence of microwaves) phenomenon which relates ZdRS to the maxima of magnetooscillations at zero dc field. The vanishing of $r_{\omega}$ of a similar nature with ZdRS evolving from the maxima of oscillationshas also been observed in the absence of radiation: in particular, the emergence of ZdRS from the maxima of strongly developed $\mathrm{SdH}$ oscillations was demonstrated by Bykov et al. (2007). The ZdRS was also found to emerge from the maxima of MISO (Bvkov et al., 2010d; Gusev et al., 2011; Wiedmann et al., 2011a).

\section{Frequency mixing for magnetooscillations}

Analysis of the experimental data in Fig. $36 \mathrm{~b}$ led Hatke et al. (2008b) to conclude that the local maxima of $r_{\omega}$ in the $\epsilon_{\mathrm{ac}}-\epsilon_{\mathrm{dc}}$ plane occur at the points given by

$$
\epsilon_{\mathrm{ac}}+\epsilon_{\mathrm{dc}} \simeq n, \quad \epsilon_{\mathrm{ac}}-\epsilon_{\mathrm{dc}} \simeq m-1 / 2,
$$

where $n$ and $m$ are integers [the first condition was established in earlier work by Zhang et al. (2007c)]. Hatke et al. (2008b) also argued that Eqs. (167) point to the key role played by backscattering off disorder in shaping the oscillations in Fig. 36b (similar to HIRO in the limit of $\epsilon_{\mathrm{dc}} \gg 1$ ). Within a qualitative picture proposed by Hatke et al. (2008b), $r_{\omega}$ has a maximum when disorder-induced scattering, accompanied by shifts of the cyclotron orbit in real space by $2 R_{c}$, is maximized for shifts in the direction of the dc field (first condition) and minimized for shifts in the opposite direction (second condition).

As the calculation by Khodas and Vavilov (2008) shows, the above picture is qualitatively accurate (to first order in $\mathcal{P}$ and for $\epsilon_{\mathrm{ac}}>\epsilon_{\mathrm{dc}}$ ) in describing the mechanism of "frequency mixing" of MIRO and HIRO; however, it misses an important microwave-induced correction to the amplitude of HIRO which arises at the same order in $\mathcal{P}$. Specifically, to order $\mathcal{O}(\mathcal{P})$ and at $\epsilon_{\mathrm{dc}} \gg 1$, the oscillatory correction to the resistivity $\delta r_{\omega}=r_{\omega}-\rho_{\mathrm{D}}$ for overlapping LLs reads (Khodas and Vavilov, 2008)

$$
\begin{aligned}
& \delta r_{\omega} / \rho_{\mathrm{D}}=\left(16 \delta^{2} / \pi \epsilon_{\mathrm{dc}}\right)\left(\tau / \tau_{\pi}\right)\left(C_{0}+C_{+}+C_{-}\right) \\
& C_{0}=(1-2 \mathcal{P}) \epsilon_{\mathrm{dc}} \cos 2 \pi \epsilon_{\mathrm{dc}} \\
& C_{ \pm}=\mathcal{P}\left(\epsilon_{\mathrm{dc}} \pm \epsilon_{\mathrm{ac}}\right) \cos 2 \pi\left(\epsilon_{\mathrm{dc}} \pm \epsilon_{\mathrm{ac}}\right)
\end{aligned}
$$

The frequency-mixing terms $C_{ \pm}$are seen to be exactly canceled by the linear-in- $\mathcal{P}$ correction to the amplitude of HIRO in $C_{0}$ at integer $\epsilon_{\mathrm{ac}}$ (in which case the oscillatory correction $\delta r_{\omega}$ reduces to the plain HIRO).

Moreover, Eqs. (167) can be obtained from Eqs. (168) only if the modified HIRO, described by the term $C_{0}$, 

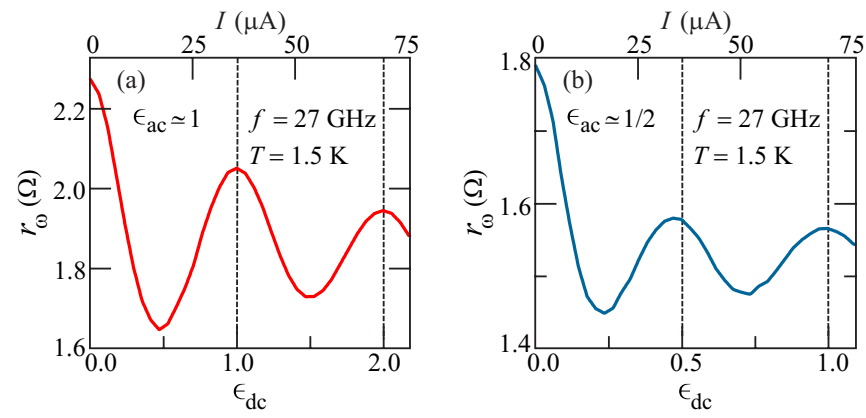

FIG. 37 Differential resistivity $r_{\omega}$ versus $\epsilon_{\mathrm{dc}}$ (bottom axis) and the direct current $I$ (top axis) at (a) $\epsilon_{\mathrm{ac}} \simeq 1 / 2$ and (b) $\epsilon_{\mathrm{ac}} \simeq 1$. The data were obtained at $f=27 \mathrm{GHz}$ and $T=1.5 \mathrm{~K}$ in a $100 \mu \mathrm{m}$ wide Hall bar sample with $n_{e} \simeq 3.8 \times 10^{11} \mathrm{~cm}^{-2}$ and $\mu \simeq 1.3 \times 10^{7} \mathrm{~cm}^{2} /$ v s. From Hatke et al. (2008a).

are neglected. In general, the location of the extrema of $r_{\omega}$ given by Eqs. (168), and the whole picture of the oscillations for that matter, depend in an essential way on $\mathcal{P}$. In particular, the most characteristic feature in Figs. 36 the vanishing of the oscillations at half-integer $\epsilon_{\mathrm{ac}}$ - can be obtained, within the theoretical framework on which Eqs. (168) are based, at special values of $\mathcal{P}$ only. Specifically, Eqs. (168) yield for half-integer $\epsilon_{\mathrm{ac}}$ :

$$
\delta r_{\omega} / \rho_{\mathrm{D}}=\left(16 \delta^{2} / \pi\right)\left(\tau / \tau_{\pi}\right)(1-4 \mathcal{P}) \cos 2 \pi \epsilon_{\mathrm{dc}} .
$$

The amplitude of the oscillations in Eq. (169) is seen to be reduced by microwaves. In fact, extending the calculation of $\delta r_{\omega}$ for $\epsilon_{\mathrm{dc}} \gg 1$ to arbitrary $\mathcal{P}$ (Khodas et al., 2010; Khodas and Vavilov, 2008) gives for half-integer $\epsilon_{\text {ac }}$ Eq. (169) with the factor $1-4 \mathcal{P}$ being substituted by the Bessel function $J_{0}(4 \sqrt{\mathcal{P}})$ [see Eq. (170) below]. The nodes of the oscillations of $r_{\omega}$ as a function of $\epsilon_{\mathrm{dc}}$ at half-integer $\epsilon_{\mathrm{ac}}$ are thus only reproduced at zeros ${ }^{20}$ of $J_{0}(4 \sqrt{\mathcal{P}})$. Given the accuracy of the reported nodes at half-integer $\epsilon_{\mathrm{ac}}$, it appears that the data in Fig. 36 were likely obtained in the very close vicinity of a zero of $J_{0}(4 \sqrt{\mathcal{P}})$.

\section{Fractional HIRO}

The nonlinear response of a 2DEG to the dc field was also investigated experimentally in the regime of fractional MIRO, in the vicinity of the most prominent fraction $\epsilon_{\mathrm{ac}}=1 / 2$, by Hatke et al. (2008a). Remarkably, the experiment revealed the emergence of fractional HIRO at $\epsilon_{\mathrm{ac}}=1 / 2$, with "frequency-doubling" for the oscillations of $r_{\omega}$ as a function of $\epsilon_{\mathrm{dc}}$. As seen in Fig. 37 the overall behavior of $r_{\omega}$ at $\epsilon_{\mathrm{ac}}=1 / 2$ in the interval

20 A reversal of the sign of the oscillations with $\epsilon_{\mathrm{dc}}$ when $\mathcal{P}$ reaches a certain threshold, reported by Zudov et al. (2009), may provide a valuable calibration point for the microwave intensity seen by the $2 \mathrm{DEG}$, which is difficult to obtain from other experiments. $0<\epsilon_{\mathrm{dc}}<1$ closely replicates that at the $\mathrm{CR}$ in the interval $0<\epsilon_{\mathrm{dc}}<2$-with the period of the oscillations in $\epsilon_{\mathrm{dc}}$ at $\epsilon_{\mathrm{ac}}=1 / 2$ being half that at $\epsilon_{\mathrm{ac}}=1$ (which means that the period of the oscillations of $r_{\omega}$ as a function of the direct current is the same).

A description of the fractional HIRO in a microwaveexcited 2DEG was suggested by Lei (2009) in terms of a postulated ansatz which assumes an infinitely fast thermal equilibration in the frame moving with the drift velocity (see footnote 19). Lei (2009) argued that the maxima of $r_{\omega}$ should obey the condition $\epsilon_{\mathrm{dc}}+n \epsilon_{\mathrm{ac}}=m$ with integer $n$ and $m$, and the numerically obtained solution to the ansatz equation yielded a picture similar to the one in Fig. 37 however, the dependence of the strength and shape of the oscillations on the major parameters, such as $\mathcal{P}$ or the degree of overlap of LLs, remained unclarified.

On the other hand, the observed frequency-doubling for HIRO may be a signal of the inelastic mechanism of nonequilibrium magnetooscillations. Indeed, in overlapping LLs, a controlled calculation by Khodas and Vavilov (2008) at order $\delta^{2}$ and at first order in $\mathcal{P}$ in the collision integral showed that, while the displacement mechanism is dominant in the limit of $\epsilon_{\mathrm{dc}} \gg 1$, the inelastic mechanism for $\tau_{\text {in }} / \tau \gtrsim 1$ is equally important at $\epsilon_{\mathrm{dc}} \sim 1$ (see also Sec. V.C) and produces strong subharmonics in the dependence of $r_{\omega}$ on $\epsilon_{\mathrm{dc}}$-in contrast to the displacement mechanism at this order in $\delta$. Importantly, the strength of the subharmonics in the presence of radiation depends on $\mathcal{P}$ and oscillates with $\epsilon_{\mathrm{ac}}$ (Khodas and Vavilov, 2008). In particular, for overlapping LLs, the subharmonic with a period in $\epsilon_{\mathrm{dc}}$ equal to $1 / 2$ is as strong at $\epsilon_{\mathrm{ac}}=1 / 2$, $\epsilon_{\mathrm{dc}} \sim 1$, and $\mathcal{P} \sim 1$ as the harmonic with period 1 . In the case of separated LLs, the half-integer MIRO and half-integer HIRO are also likely to strengthen each other in analogy with the theory of two-photon absorption (Sec. VI.A). Further theoretical work is necessary to study the exact form of the interplay of the two types of fractional oscillations.

\section{AC field-periodic oscillations}

In Sec. VI.B.3 the focus was on the regime of a moderate microwave power $(\mathcal{P} \lesssim 1)$. The nonlinear mixing of HIRO and MIRO in the case of high-power radiation $(\mathcal{P} \gg 1)$ results in the emergence of oscillations of $r_{\omega}$ as a function of $\mathcal{P}$ (in addition to the oscillations in $\epsilon_{\mathrm{dc}}$ and $\epsilon_{\mathrm{ac}}$ ). These were observed (Khodas et al., 2010) as a series of oscillations grouped around the CR and decaying away from it, with much less pronounced but similar behavior also detected around $\epsilon_{\mathrm{ac}}=2$. In contrast to the integer MIRO, which have one maximum and one minimum per unit interval of $\epsilon_{\mathrm{ac}}$ (Fig. [38 a), or the fractional MIRO with their oscillatory features around rational values of $\epsilon_{\mathrm{ac}}$, these oscillations exhibit multiple maxima and minima around the CR (and its second harmonic) with a period which depends on $\mathcal{P}$, as shown in Fig. 38b.

A theoretical description by Khodas et al. (2010); 


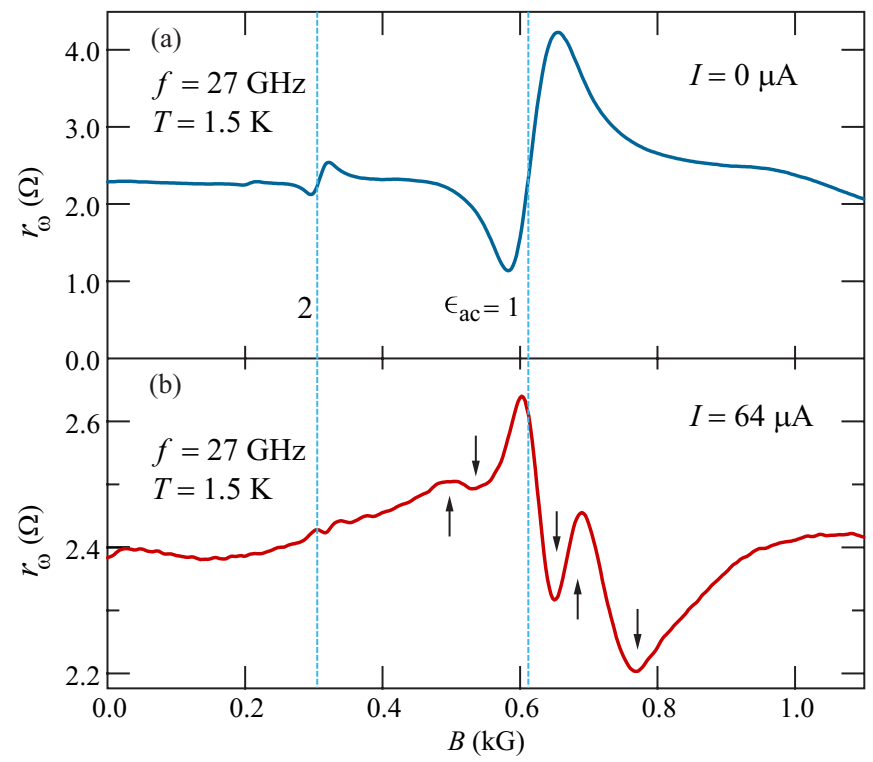

FIG. 38 Differential resistivity $r_{\omega}(B)$ (a) for zero direct current $I$ and (b) for $I=64 \mu \mathrm{A}$. The vertical lines mark the position of the $\mathrm{CR}$ and its second harmonic. Parameters of the sample, frequency $f=27 \mathrm{GHz}$, and temperature $T=1.5 \mathrm{~K}$ are the same as in Fig. 37. Adapted from Khodas et al. (2010).

Khodas and Vavilov (2008) of the reported oscillations in $\mathcal{P}$ gives for the oscillatory correction $\delta r_{\omega}$ at order $\delta^{2}$ for $\epsilon_{\mathrm{dc}} \gg \max \{1, \sqrt{\mathcal{P}}\}$ :

$$
\begin{aligned}
& \frac{\delta r_{\omega}}{\rho_{\mathrm{D}}}=\frac{16 \delta^{2}}{\pi \epsilon_{\mathrm{dc}}} \frac{\tau}{\tau_{\pi}}\left[\epsilon_{\mathrm{dc}} \cos \left(2 \pi \epsilon_{\mathrm{dc}}\right) J_{0}\left(4 \sqrt{\mathcal{P}} \sin \pi \epsilon_{\mathrm{ac}}\right)\right. \\
& \left.-2 \epsilon_{\mathrm{ac}} \sin \left(2 \pi \epsilon_{\mathrm{dc}}\right) \cos \left(\pi \epsilon_{\mathrm{ac}}\right) \sqrt{\mathcal{P}} J_{1}\left(4 \sqrt{\mathcal{P}} \sin \pi \epsilon_{\mathrm{ac}}\right)\right],
\end{aligned}
$$

which generalizes Eqs. (168) to the case of large $\mathcal{P}$, with the Bessel functions $J_{0,1}$ oscillating with a period which depends on $\mathcal{P}$ and $\epsilon_{\text {ac }}$. Oscillations in $\mathcal{P}$ of this form are a characteristic signature of multiphoton transitions between stationary states of a periodically (in time) driven electron system. Namely, the absorption rate for $N$ photon transitions between these states is proportional ${ }^{21}$ to $J_{N}^{2}[2 \sqrt{\mathcal{P}} \sin (\theta / 2)]$, where $\theta$ is the angle between the initial and final directions of the velocity (in the quasiclassical formulation) (Khodas et al., 2010; Lei, 2009). In the limit $\epsilon_{\mathrm{dc}} \gg 1$, where the main contribution to HIRO comes from $\theta \simeq \pi$, summing over $N$ (Khodas et al., 2010) reproduces Eq. (170). The characteristic number of photons $N$ in the transitions that yield Eq. (170) for $\mathcal{P} \gg 1$

21 A similar structure arises in a variety of closely related problems, e.g., in studying the multiphoton $\mathrm{CR}$ in three dimensions (Seelv, 1974) or the dynamical Franz-Keldysh effect (Johnsen and Jauho, 1998).
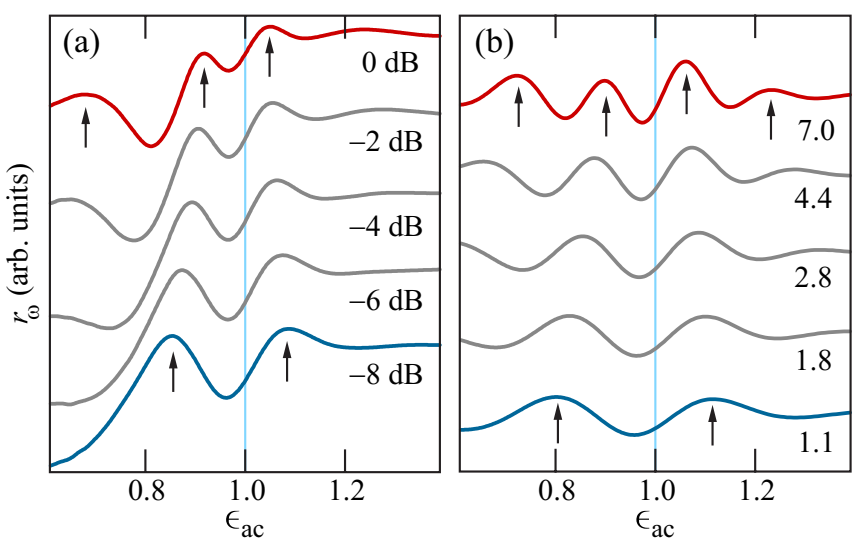

FIG. 39 (a) Measured and (b) calculated differential resistivity $r_{\omega}$ versus $\epsilon_{\mathrm{ac}}$ for $I=54 \mu \mathrm{A}$ and different microwave intensities. The traces are labeled according to (a) the attenuation levels and (b) the value of $\mathcal{P}$. Parameters of the sample, frequency $f=27 \mathrm{GHz}$, and temperature $T=1.5 \mathrm{~K}$ are the same as in Figs. 37 and 38 . From Khodas et al. (2010).

is $^{22} 2 \sqrt{\mathcal{P}}$. This conclusion is further corroborated by the observation that $r_{\omega}$ from Eq. (170) in the limit $\mathcal{P} \gg 1$ oscillates, as a function of $\epsilon_{\mathrm{ac}}$ in the vicinity of integer $\epsilon_{\mathrm{ac}}$, with a period $1 / 2 \sqrt{\mathcal{P}}$. The experimental data (Khodas et al., 2010), shown in Fig. 39a, demonstrate that the oscillations of $r_{\omega}$ with $\epsilon_{\mathrm{ac}}$ become more rapid with increasing microwave power, in close agreement with the result of Eq. (170), shown in Fig. 39 b.

\section{OUTLOOK}

\section{A. Perspectives}

Here we briefly outline several particularly promising directions of ongoing and future research in the field of nonequilibrium magnetotransport phenomena in semiconductor structures.

\section{Domain structure of ZRS and ZdRS}

The present status of research in this area has been reviewed in Sec. IV] The physics of the broken-symmetry states with spontaneously formed domains remains one

\footnotetext{
${ }^{22}$ More specifically, for $1 / \mathcal{P}^{1 / 2} \ll\left|\bar{\epsilon}_{\mathrm{ac}}\right|$, where $\bar{\epsilon}_{\mathrm{ac}}=\epsilon_{\mathrm{ac}}-n$ and $n$ is the nearest integer, the sum over $N$, which is of the type $\sum_{N} J_{N}^{2}(2 \sqrt{\mathcal{P}})\left(\epsilon_{\mathrm{dc}}+N \epsilon_{\mathrm{ac}}\right) \cos \left[2 \pi\left(\epsilon_{\mathrm{dc}}+N \epsilon_{\mathrm{ac}}\right)\right]$ and contains the factor oscillating in $N$ with a period $1 /\left|\bar{\epsilon}_{\text {ac }}\right|$, is determined by a close vicinity of the sharp peak in $J_{N}^{2}(2 \sqrt{\mathcal{P}})$ at $N=2 \sqrt{\mathcal{P}}$ whose width is of order $\mathcal{P}^{1 / 6}$. The peak falls off exponentially on the side of larger $N$ and as $(2 \sqrt{\mathcal{P}}-N)^{-1 / 2}$ on the other side. For $1 / \mathcal{P}^{1 / 2} \ll\left|\bar{\epsilon}_{\text {ac }}\right| \ll 1 / \mathcal{P}^{1 / 6}$, the main contribution to $\delta r_{\omega}$ comes from a narrow interval of positive $2 \sqrt{\mathcal{P}}-N$ whose width is of order $1 /\left|\bar{\epsilon}_{\mathrm{ac}}\right|$.
} 
of the central research directions in the field. Particularly important open questions include the character of the transition into ZRS, the corresponding critical behavior (in the case when the transition is continuous) beyond the mean-field picture, the residual resistance of a finitesize system, the origin of the experimentally observed Arrhenius-like temperature dependence of the ZRS resistivity, the effect of thermal and nonequilibrium noise on ZRS, as well as dynamics of the domain structure.

\section{Giant photoresponse at the second harmonic of the cyclotron resonance}

Recent experiments on ultra-high mobility structures reported a narrow spike at $\omega \simeq 2 \omega_{\mathrm{c}}$ superposed on the $n=2$ maximum of MIRO (Dai et al., 2010; Hatke et al., $2011 \mathrm{~b})$. The height of the spike was several times (up to an order of magnitude) larger than the height of other MIRO maxima. Dai et al. (2010) also observed an enhancement of higher-order even MIRO maxima ( $n=4,6,8)$, see Fig.40. It is important to note that these effects were observed in samples which also show a very strong negative MR (also in the absence of microwaves) attributed to the quasiclassical memory effects, see Sec.II.B.2, which indicates a possible connection between these phenomena. As a matter of fact, the memory effects that are responsible for the QCMR may also produce spikes in the photoconductivity at the CR harmonics, see Secs. II.B.2 I.B.3, and ПI.B.5. However, it is unclear why only even harmonics (and, most prominently, the one at $n=2$ ) are enhanced. Further theoretical work is thus needed.

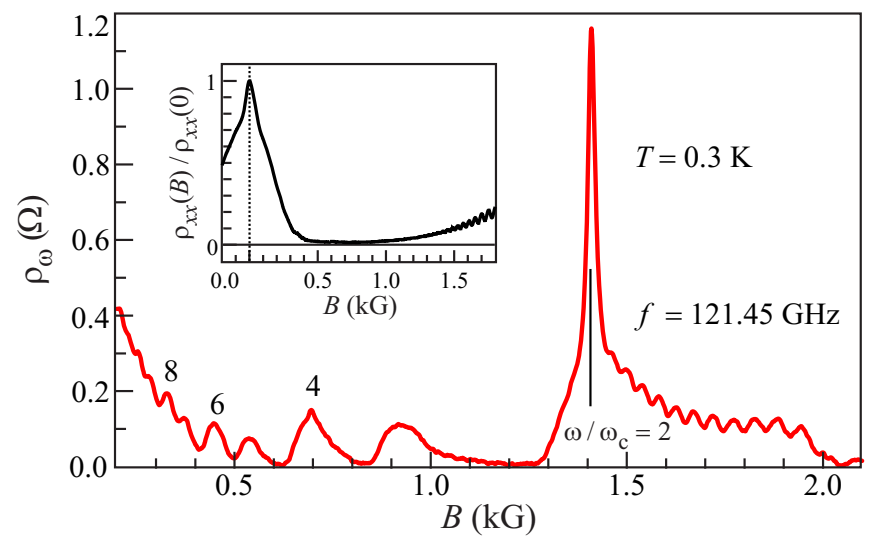

FIG. 40 Magnetoresistivity measured at $f=121.45 \mathrm{GHz}$ and $T=0.3 \mathrm{~K}$ in a Hall bar sample with $n_{e} \simeq 2.9 \times 10^{11} \mathrm{~cm}^{-2}$ and $\mu \simeq 3.0 \times 10^{7} \mathrm{~cm}^{2} / \mathrm{V} \mathrm{s}$. The dark resistivity, normalized to its value at $B=0$, is shown in the inset. Adapted from Dai et al. (2010).

\section{Nonequilibrium phenomena in separated Landau levels}

Nonequilibrium phenomena in the regime of fully separated LLs deserve further study. On the theoretical side, the theory for mixed disorder, which is the type of disorder characteristic of most of the experimentally relevant samples (see Secs. III and V]), needs to be extended into this regime. On the experimental side, this corresponds to studying the nonequilibrium phenomena in stronger magnetic fields. It is further interesting to see how the phenomena discussed in this review evolve when entering the QH regime, see also Sec. VII.A.7.

\section{Nonequilibrium transport at low temperature}

Most of the experiments and theories presented in this review focussed on the temperature regime where $\mathrm{SdH}$ oscillations are suppressed. The interplay of the nonequilibrium physics and $\mathrm{SdH}$ oscillations at lower $T$ is also of interest. A remarkable phenomenon of this kind that was studied both experimentally (Bvkov et al., 2007; Kalmanovitz et al., 2008; Studenikin et al., 2012; Zhang et al., 2009, 2007a; Zudov et al., 2011) and theoretically (Dmitriev, 2011) is the dc field-induced inversion of the phase of $\mathrm{SdH}$ oscillations. In particular, Bykov et al. (2007) reported the formation of ZdRS that arise, as the strength of the dc field increases, from the maxima of SdH oscillations, see Sec. V.D. A similar effect on the $\mathrm{SdH}$ oscillations by the microwave field was observed by Mani (2004b).

\section{Spin-related phenomena}

In the regime of strongly developed SdH oscillations, the spin degree of freedom starts to play an important role. Romero et al. (2008) explored the effect of a parallel magnetic field $B_{\|}$on ZdRS in this regime (Sec. IV.D) and found that the ZdRS are destroyed for sufficiently strong $B_{\|}$. The result was explained (Romero et al., 2008) within the kinetic equation formalism described in Secs. IIIV in terms of a Zeeman splitting-induced modification of the oscillatory component of the DOS. Studenikin et al. (2012) further extended the study of the effect of Zeeman splitting on nonlinear magnetotransport to the regime of strong magnetic fields normal to the 2DEG. Still, spin-related phenomena in nonequilibrium magnetotransport remain largely unexplored. This research direction overlaps with the investigations of the regime of separated LLs (Sec. VII.A.3) and of the $\mathrm{QH}$ regime (Sec. VII.A.7).

\section{Polarization dependence of MIRO}

As discussed in Sec.III.B.1.b the relative orientation of the dc field and the linearly polarized microwave field affects considerably the MIRO amplitude within the 
framework of the displacement mechanism, while the inelastic contribution is insensitive to the direction of the linear polarization. Mani (2004a); Mani et al. (2002); Wiedmann et al. (2011a) reported equal MIRO signal strength for the microwave field polarized along and perpendicular to the current direction, which is consistent with the dominance of the inelastic contribution. On the other hand, a recent experimental study (Mani et al., 2011) reported a pronounced difference of the MIRO amplitude for two directions of the linear polarization, indicating the relevance of the displacement contribution. Systematic experimental study of the dependence of this effect on the temperature and microwave power would permit a quantitative comparison with theoretical predictions (see Sec.III.B.1.g).

Smet et al. (2005) used an optical setup in order to study MIRO induced by circularly polarized microwaves. According to the theories presented in Sec. III the amplitude of MIRO depends on the direction of circular polarization through the factor $1 /\left[\left(\omega \pm \omega_{c}\right)^{2}+1 / \tau^{2}\right]$, where the minus (plus) sign corresponds to active (inactive) polarization. Surprisingly, Smet et al. (2005) reported very similar results for two directions of circular polarization, with a difference being much smaller than expected from this factor, see Fig. 41, To understand a possible reason for the failure to see a large effect after changing the direction of circular polarization of the incoming radiation, it is important to take into account that the electric field in Eqs. (74), (77), and (82) is the total electric field acting on electrons in the sample.

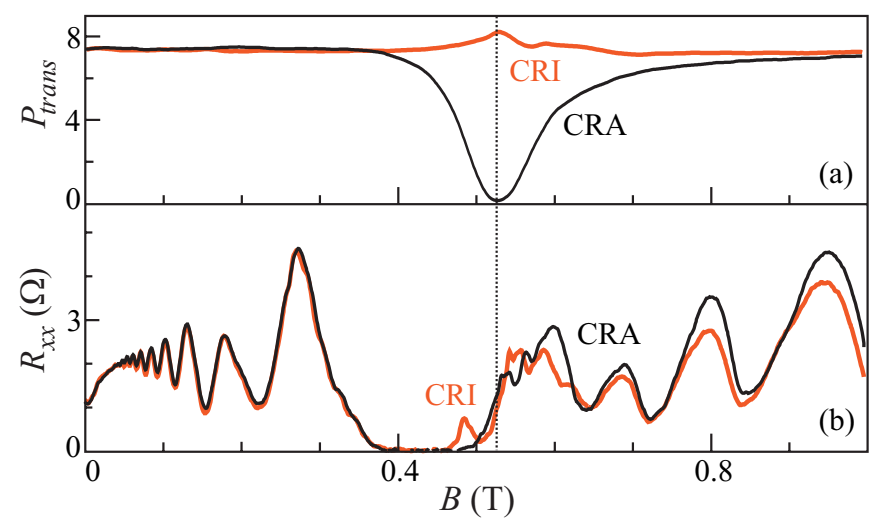

FIG. 41 (a) Transmitted power (arb. units) and (b) magnetoresistivity measured at $f=200 \mathrm{GHz}$ and $T \simeq 1.7 \mathrm{~K}$ in a van der Pauw sample with $n_{e} \simeq 2.6 \times 10^{11} \mathrm{~cm}^{-2}$ and $\mu \simeq 1.8 \times 10^{7} \mathrm{~cm}^{2} / \mathrm{Vs}$ for both active (CRA) and inactive (CRI) senses of circular polarization. Adapted from Smet et al. (2005).

The total field is different from the source field because of screening by the 2DEG, see Secs. II.C.3, III.B.1.C. This leads to a strong broadening of the CR: the above factors are modified to $1 /\left[\left(\omega \pm \omega_{c}\right)^{2}+\gamma^{2}\right]$, where $\gamma=$ $2 \pi e^{2} n_{e} / m c n_{r}$ [Eq. (57)] for $\gamma \gg 1 / \tau$. For a typical concentration $n_{e}=3 \times 10^{11} \mathrm{~cm}^{-2}$ and the effective refractive index $n_{r}=(\sqrt{12.4}+1) / 2 \simeq 2.3$ for the case of a GaAs- based structure one finds $\gamma / 2 \pi \simeq 17 \mathrm{GHz}$, which is comparable to characteristic microwave frequencies. While this explains why no sharp resonance features is experimentally observed near $\omega=\omega_{c}$, this is not sufficient to explain why the difference in the amplitude of the MIRO harmonics with $n=2,3,4$ for two directions of the circular polarization in Smet et al. (2005) was within a few percent (Fig. 41). Indeed, since $\gamma$ is several times smaller than the frequencies $\omega / 2 \pi=100 \div 250 \mathrm{GHz}$ used in this experiment, one would expect a much larger difference (a factor of about 9 for $n=2$ and of about 4 for $n=3$, etc.). This discrepancy can be most likely attributed to the fact that the metallic electrodes and other elements of the sample setup may strongly affect the polarization of the electric field. In particular, Mikhailov and Savostianova (2006) showed, for a simple geometry, that metallic contacts may strongly reduce the degree of circular polarization of microwaves [the actual geometry of the experiment (Smet et al., 2005) is much more complex, and a quantitative evaluation of the polarization of the actual electric field would require a numerical modeling of the whole setup].

In fact, close inspection of the transmission data shown in Fig. 41a [see also Fig. 1 of Smet et al. (2005)] provides additional support to this explanation. While the data do show, as expected, a CR dip around $\omega_{c}=\omega$ for the active polarization, the $-1 /\left(\omega-\omega_{c}\right)^{2}$ tail of the Lorentzian for the active polarization is not observed when $\left|\omega_{c}\right|$ becomes smaller than $\omega$ by more than $\gamma$. Instead, the data for both polarizations of the incoming wave become practically identical, with the transmission amplitude for the active polarization being even slightly higher. This suggests that, in this range of magnetic fields, the polarization of the incoming radiation was essentially immaterial for the actual polarization of the electric field. Since this is exactly the range of magnetic fields where MIRO are measured, this observation is consistent with the fact that the MIRO signal was almost identical for both directions of the circular polarization of the incoming wave.

It appears that the presence of nearby metallic contacts and, more generally, the effects of a finite sample geometry may strongly affect the actual polarization of microwaves, thus seriously complicating the study of the polarization dependence of MIRO. Devising an experimental setup that would be free from these drawbacks remains a challenge for future work.

\section{Nonequilibrium magnetotransport in the integer and fractional quantum Hall regime}

It is natural to ask whether the phenomena described in this review have their counterparts in the $\mathrm{QH}$ regime, both on a $\mathrm{QH}$ plateau and near a $\mathrm{QH}$ transition. In this respect, it is worth recalling another nonequilibrium phenomenon - the breakdown of the $\mathrm{QH}$ effect at a high current density - that has been extensively studied, see Nachtwei (1999) for a review. While this is an 
essentially different phenomenon, certain aspects bear similarity with the MIRO and HIRO and, especially, ZRS and ZdRS physics (the nonequilibrium phase transition between states with zero and finite resistivity in a transverse magnetic field; the inhomogeneous spatial distribution of the electric field and current; the importance of relaxation processes; etc.). Recent experiments (Nachtwei et al., 2008; Stellmach et al., 2007) addressed the photoresponse of QH structures on both sides of the breakdown bias. It remains to be seen whether there is a deeper connection between the two classes of phenomena.

It would be further highly interesting to observe phenomena analogous to those reviewed here near halffilling of the lowest LL, where transport is associated with composite fermions (CFs) (Heinonen, 1998; Jain, 2007). A possibility to observe MIRO of CFs was discussed by Park (2004). There is, however, a serious difficulty related to the fact that CFs are much more strongly scattered by disorder than electrons. The dominant type of disorder for CFs is an effective random magnetic field produced by the random scalar potential through the flux attachment (Halperin et al., 1993). The damping of quantum magnetooscillations induced by the random magnetic field is much stronger than the one $\left(\propto e^{-\pi / \omega_{c} \tau_{\mathrm{q}}}\right)$ characteristic of the regime of low magnetic fields (Aronov et al., 1995; Evers et al., 1999; Mirlin et al., 1996, 1998). This appears to be an essential obstacle for the observation of pronounced MIRO or HIRO in this regime. On the other hand, while the DOS oscillations around $\nu=1 / 2$ are very strongly damped, one does observe conductivity oscillations which are of a different origin: they are induced by quantum localization of CFs, leading eventually to the fractional QH effect (Evers et al., 1999; Mirlin et al., 1998). An intriguing question, deserving theoretical and experimental investigation, is whether these oscillations would be sufficient to induce MIRO or HIRO under nonequilibrium conditions.

\section{B. Nonequilibrium magnetotransport phenomena in other 2D systems}

\section{Electrons on liquid helium}

Electrons on a surface of liquid helium represent a remarkable 2D electronic system with parameters strongly different from those of 2DEGs in semiconductor structures, see Andrei (1997); Grimes (1978); Monarkha and Kono (2004) for reviews. First, because of a very low density, correlations play an essential role: in the zero-temperature limit this system would be a Wigner crystal. Second, the electron gas on helium becomes nondegenerate already for very low temperatures. Finally, relaxation times at Kelvin temperatures are several orders of magnitude longer than in semiconductor 2DEGs. Quasiclassical magnetotransport in this system has been studied both experimentally and theoretically during the last two decades (Dykman et al., 1997, 1993;
Dykman and Prvadko, 2003; Lea and Dykman, 1998; Monarkha and Kono, 2004). The focus of a more recent activity was on the photoresponse of the electron fluid. A characteristic feature of the photoresponse is resonant absorption at a frequency of order $100 \mathrm{GHz}$ (Konstantinov et al., 2009, 2012b, 2007, 2008a, b), corresponding to the transition from the lowest to the first excited subband (i.e., the first excited state for motion in the direction transverse to the surface). It is worth noting that the conductivity of electrons on the helium surface is measured in a contactless way, by capacitively coupling the $2 \mathrm{D}$ electron system to electrodes and measuring the complex conductance (admittance) of the circuit.

In recent experiments (Konstantinov and Kono, 2009, 2010), the resonant intersubband excitation measurements were performed in a transverse magnetic field. Remarkably, the system showed $1 / B$-periodic magnetoconductance oscillations and zero-conductance states (the experiments were performed in the Corbino-disk geometry), similar to those found in the semiconductor 2DEG (Secs. III, IV). The similarity includes the same periodicity and phase, as well as a strong temperature dependence. Further, Konstantinov et al. (2012a) reported spontaneous currents (in the absence of driving voltage) in the regime of zero-conductance states. An explanation of these oscillations put forward by Konstantinov and Kono (2009, 2010); Monarkha (2011a, b) is analogous to the MIRO theory (Sec III) and combines features of both the inelastic and displacement mechanisms. Specifically, it was proposed that the resonant microwave absorption leads to a strongly nonequilibrium occupation of the first excited subband, which is followed by a quasielastic transition from the lowest LL of the first excited subband to one of higher LLs of the lowest subband.

The above observations appear truly remarkable as they show that the nonequilibrium phenomena in high LLs, discussed in the review, are also relevant to strongly interacting and nondegenerate $2 \mathrm{D}$ electron systems. On the other hand, a systematic theory of the nonequilibrium magnetotransport phenomena in the electron liquids on helium remains to be developed. In particular, one has to clarify the role of resonant absorption to the excited subband, the relative weight of the contributions of different mechanisms, relevant relaxation processes, etc.

\section{Graphene}

The experimental breakthrough in fabrication of graphene (Novoselov et al., 2004) has brought a new 2D system with fascinating properties into condensedmatter physics (Geim and Novoselov, 2007). The hallmark of graphene is the massless Dirac-fermion character of charge carriers. It manifests itself, in particular, in the unconventional QH effect in both graphene (Novoselov et al., 2005; Zhang et al., 2005) and graphene bilayers (McCann and Fal'ko, 2006; Novoselov et al. 
2006). The Dirac spectrum implies also a linearly vanishing DOS at the neutrality point, i.e., the effective mass vanishes as $\sqrt{\left|n_{e}\right|}$ as a function of the electron concentration or, equivalently, of the gate voltage. As a result, the LLs are not equidistant, with the distance between them (cyclotron frequency) being strongly enhanced near the Dirac point. One of the manifestations of this is the observation of the $\mathrm{QH}$ effect up to room temperature (Novoselov et al., 2007). For a recent review of the electronic properties of graphene see (Castro Neto et al., 2009). One of the most active directions in the graphene research is devoted to the optoelectronic properties of graphene, including a variety of prospective applications (photodetectors, light emitters, solar cells, lasers, etc) (Bonaccorso et al., 2010; Mueller et al., 2010).

Spectroscopy studies of graphene in a transverse magnetic field confirmed the Dirac-fermion LL spectrum (Sadowski et al., 2006). Neugebauer et al. (2009) presented a spectroscopic manifestation of the exceptionally high purity of graphene samples: the LL quantization was observed at $B$ as low as $1 \mathrm{mT}$ and $T$ up to $50 \mathrm{~K}$. For a review of LL spectroscopy of graphene see Orlita and Potemski (2010).

The properties of graphene make it a very interesting and promising system for studying nonequilibrium physics in quantizing magnetic fields. Several differences compared to the semiconductor systems may be foreseen. First, according to the above, one can expect that in high-quality graphene samples with the chemical potential located near the Dirac point the quantum phenomena will become pronounced at relatively high $T$ and relatively low $B$. Second, the nonequidistant character of LLs may lead to a modification of the functional dependence of magnetooscillations on $T$ and the strength of disorder. In fact, a first experiment in this direction appeared recently: Tan et al. (2011) studied SdH oscillations in graphene under dc bias and observed a phase inversion analogous to that found in semiconductor structures, see Sec. VII.A.4

\section{ACKNOWLEDGMENTS}

We are grateful to Y. Adamov, I.L. Aleiner, H.-S. Chiang, S.I. Dorozhkin, R.R. Du, A.L. Efros, F. Evers, O.M. Fedorych, R. Gellmann, I.V. Gornyi, J.A. Gupta, A.T. Hatke, M. Khodas, T.A. Knuuttila, F. von Oppen, I.V. Pechenezhskii, L.N. Pfeiffer, I.V. Ponomarev, M. Potemski, J.L. Reno, J.A. Simmons, S.A. Studenikin, M.G. Vavilov, Z.R. Wasilevski, K.W. West, J. Wilke, P. Wölfle, C.L. Yang, Z.Q. Yuan, and W. Zhang for fruitful collaborations on the topics included in this review. We also thank many of our colleagues, in particular, A. Auerbach, A.A. Bykov, K. von Klitzing, I.V. Kukushkin, R.G. Mani, O.E. Raichev, M.E. Raikh, V.I. Ryzhii, B.I. Shklovskii, J.H. Smet, R.A. Suris, S.A. Vitkalov, V.A. Volkov, and R.L. Willett, for illuminating discussions over the past years. This work was supported by DFG, DFG-CFN, DFG-RFBR, DFG-SPP "Halbleiter Spintronik", DOE Grant No. DE-SC002567, and NSF Grant No. DMR-0548014.

Note added in proof.- Several important developments occurred after the submission of this review. References to some of them are added to the main text, others are listed below.

- In a series of papers (Dietrich et al., 2012a, b; Dmitriev et al., 2012), the transport properties of a 2DEG in a GaAs quantum well surrounded by AlAs/GaAs superlattices were investigated. A peculiar feature of these structures is a substantial concentration of mobile (but highly resistive) $X$ electrons in the AlAs layers. These layers serve as additional screening gates and induce a spatially inhomogeneous electron density profile across the Hall bar when a direct current is driven through the 2DEG. The obtained results include the suppression of the transport and quantum scattering rates (particularly strong for the latter) due to the screening by $X$ electrons (Dmitriev et al., 2012), as well as the observation of a current-induced modulation of SdH oscillations (Dietrich et al., 2012a) and MISO (Dietrich et al., 2012b) in the nonlinear regime resulting from the electron density variation induced by the $X$-electron layer. Unlike HIRO, the period of these oscillations is independent of $B$.

- SdH oscillations in ultrahigh mobility quantum wells in tilted magnetic fields were measured by Hatke et al., 2012b at very high tilt angles. The data were explained in terms of the dependence of the effective mass on the in-plane magnetic-field component $B_{\|}$due to a finite thickness of the quantum wells. Recall that the effect of $B_{\|}$on the scattering rates has also been observed, see Sec. III.C.3 and V.A.

- A strong photoresistance peak that originates from the magnetoplasmon resonance (MPR) superimposed on ZRS was observed by Hatke et al., $2012 \mathrm{~d}$. The experiment suggests that the contributions of MPR and MIRO to the resistivity sum up and can be independently controlled by tuning the magnetic field and microwave frequency. This makes it possible to extract separately the MPR and MIRO contributions. Similar to the bichromatic MIRO experiments (see Sec. IV.A), the extrapolation of the extracted individual contributions to the region where the observed resistance is zero yields a negative resistivity.

- For electrons on liquid helium (see Sec. VII.B.1), the theory of microwave-induced magnetoconductance oscillations was further developed (Monarkha, 2012) to include strong Coulomb interaction between electrons. The theory demonstrates the dramatic effect that slow thermal fluctuations of the electric field in the electron 
liquid have on inter-LL scattering. The theoretical results agree well with the experimental findings by Konstantinov and Kono, 2009, 2010.

\section{References}

Abrahams, E., S. V. Kravchenko, and M. P. Sarachik, 2001, Rev. Mod. Phys. 73, 251.

Abstreiter, G., J. P. Kotthaus, J. F. Koch, and G. Dorda, 1976, Phys. Rev. B 14, 2480.

Adamov, Y., I. V. Gornyi, and A. D. Mirlin, 2006, Phys. Rev. B 73, 045426.

Alferov, Z. I., 2001, Rev. Mod. Phys. 73, 767.

Alicea, J., L. Balents, M. P. A. Fisher, A. Paramekanti, and L. Radzihovsky, 2005, Phys. Rev. B 71, 235322.

Altshuler, B. L., and A. G. Aronov, 1985, in Electron-Electron Interactions in Disordered Systems, edited by A. L. Efros and M. Pollak (North-Holland, Amsterdam).

Anderson, P. W., and W. F. Brinkman, 2003, arXiv:condmat/0302129 .

Ando, T., 1974a, J. Phys. Soc. Jpn. 36, 1521.

Ando, T., 1974b, J. Phys. Soc. Jpn. 37, 1233.

Ando, T., 1975, J. Phys. Soc. Jpn. 38, 989.

Ando, T., 1982, J. Phys. Soc. Jpn. 51, 3900.

Ando, T., A. B. Fowler, and F. Stern, 1982, Rev. Mod. Phys. $\mathbf{5 4}, 437$.

Ando, T., Y. Matsumoto, and Y. Uemura, 1975, J. Phys. Soc. Jpn. 39, 279.

Ando, T., and Y. Uemura, 1974, J. Phys. Soc. Jpn. 36, 959.

Andreev, A. V., I. L. Aleiner, and A. J. Millis, 2003, Phys. Rev. Lett. 91, 056803.

Andreev, I. V., V. M. Muravev, I. V. Kukushkin, S. Schmult, and W. Dietsche, 2011, Phys. Rev. B 83, 121308(R).

Andrei, E. Y. (ed.), 1997, Two-dimensional electron systems on helium and other cryogenic substrates (Springer Verlag).

Aronov, A. G., E. Altshuler, A. D. Mirlin, and P. Wölfle, 1995, Europhys. Lett. 29, 239.

Aronov, A. G., and B. Z. Spivak, 1975, JETP Lett. 22, 101.

Auerbach, A., I. Finkler, B. I. Halperin, and A. Yacoby, 2005, Phys. Rev. Lett. 94, 196801.

Auerbach, A., and G. V. Pai, 2007, Phys. Rev. B 76, 205318.

Averkiev, N. S., L. E. Golub, S. A. Tarasenko, and M. Willander, 2001, J. Phys.: Condens. Matter 13, 2517.

Banis, T. Y., I. V. Parshelyunas, and Y. K. Pozhela, 1972, Sov. Phys. Semicond. 5, 1727.

Baskin, E. M., L. N. Magarill, and M. V. Entin, 1978, Sov. Phys. JETP 48, 365.

Bass, F. G., 1965, Sov. Phys. JETP 21, 181.

Basun, S. A., A. A. Kaplyanskii, and S. P. Feofilov, 1983, JETP Lett. 37, 586.

Beenakker, C. W. J., 1989, Phys. Rev. Lett. 62, 2020.

Beenakker, C. W. J., and H. van Houten, 1991 (NorthHolland, Amsterdam), volume 44 of Solid State Physics, p. 1.

Benedict, K. A., 1987, Nucl. Phys. B 280, 549.

Benedict, K. A., and J. T. Chalker, 1986, J. Phys. C 19, 3587.

Bergeret, F. S., B. Huckestein, and A. F. Volkov, 2003, Phys. Rev. B 67, 241303(R).

Bobylev, A. V., F. A. Maaø, A. Hansen, and E. H. Hauge, 1995, Phys. Rev. Lett. 75, 197.

Bockhorn, L., P. Barthold, D. Schuh, W. Wegscheider, and R. J. Haug, 2011, Phys. Rev. B 83, 113301.
Bogomolov, V. N., S. G. Shulman, A. G. Aronov, and G. E. Pikus, 1967, JETP Lett. 5, 169.

Bonaccorso, F., Z. Sun, T. Hasan, and A. C. Ferrari, 2010, Nature Phot. 4, 611.

Bonch-Bruevich, V. L., and S. M. Kogan, 1965, Sov. Phys. Solid State 7, 15.

Bonch-Bruevich, V. L., I. P. Zvyagin, and A. G. Mironov, 1975, Domain electrical instabilities in semiconductors (Consultants Bureau, New York).

Brézin, E., D. J. Gross, and C. Itzykson, 1984, Nucl. Phys. B 235, 24.

Buks, E., M. Heiblum, Y. Levinson, and H. Shtrikman, 1994a, Semicond. Sci. Technol. 9, 2031.

Buks, E., M. Heiblum, and H. Shtrikman, 1994b, Phys. Rev. B 49, 14790(R).

Bykov, A. A., 2008a, JETP Lett. 87, 233.

Bykov, A. A., 2008b, JETP Lett. 88, 64.

Bykov, A. A., 2008c, JETP Lett. 88, 394.

Bykov, A. A., 2010, JETP Lett. 91, 361.

Bykov, A. A., D. V. Dmitriev, I. Marchishin, S. Byrnes, and S. A. Vitkalov, 2012, Appl. Phys. Lett. 100, 251602.

Bykov, A. A., and A. V. Goran, 2009, JETP Lett. 90, 578.

Bykov, A. A., A. V. Goran, and S. A. Vitkalov, 2010a, Phys. Rev. B 81, 155322.

Bykov, A. A., D. R. Islamov, A. V. Goran, and A. I. Toropov, 2008, JETP Lett. 87, 477.

Bykov, A. A., A. K. Kalagin, and A. K. Bakarov, 2005a, JETP Lett. 81, 523.

Bykov, A. A., I. V. Marchishin, A. V. Goran, and D. V. Dmitriev, 2010b, Appl. Phys. Lett. 97, 082107.

Bykov, A. A., E. G. Mozulev, and A. K. Kalagin, 2010c, JETP Lett. 92, 379.

Bykov, A. A., E. G. Mozulev, and S. A. Vitkalov, 2010d, JETP Lett. 92, 475.

Bykov, A. A., J.-Q. Zhang, S. Vitkalov, A. K. Kalagin, and A. K. Bakarov, 2005b, Phys. Rev. B 72, 245307.

Bykov, A. A., J.-Q. Zhang, S. Vitkalov, A. K. Kalagin, and A. K. Bakarov, 2007, Phys. Rev. Lett. 99, 116801.

Cannon, E. H., F. V. Kusmartsev, K. N. Alekseev, and D. K. Campbell, 2000, Phys. Rev. Lett. 85, 1302.

Carra, P., J. T. Chalker, and K. A. Benedict, 1989, Ann. Phys. (N.Y.) 194, 1.

Castro Neto, A. H., F. Guinea, N. M. R. Peres, K. S. Novoselov, and A. K. Geim, 2009, Rev. Mod. Phys. 81, 109.

Chaplik, A. V., 1971, Sov. Phys. JETP 33, 997.

Cheianov, V. V., A. P. Dmitriev, and V. Y. Kachorovskii, 2004, Phys. Rev. B 70, 245307.

Chepelianskii, A. D., and D. L. Shepelyansky, 2009, Phys. Rev. B 80, 241308(R).

Chiu, K. W., T. K. Lee, and J. J. Quinn, 1976, Surf. Sci. 58, 182.

Choi, K. K., D. C. Tsui, and S. C. Palmateer, 1986, Phys. Rev. B 33, 8216.

Coleridge, P. T., 1990, Semicond. Sci. Technol. 5, 961.

Coleridge, P. T., 1991, Phys. Rev. B 44, 3793.

Coleridge, P. T., 1997, Semicond. Sci. Technol. 12, 22.

Coleridge, P. T., R. Stoner, and R. Fletcher, 1989, Phys. Rev. B 39, 1120.

Cooper, K. B., M. P. Lilly, J. P. Eisenstein, T. Jungwirth, L. N. Pfeiffer, and K. W. West, 2001, Solid State Commun. 119, 89.

Cross, M. C., and P. C. Hohenberg, 1993, Rev. Mod. Phys. 65, 851. 
Dai, Y., R. R. Du, L. N. Pfeiffer, and K. W. West, 2010, Phys. Rev. Lett. 105, 246802.

Dai, Y., Z. Q. Yuan, C. L. Yang, R. R. Du, M. J. Manfra, L. N. Pfeiffer, and K. W. West, 2009, Phys. Rev. B 80, 041310(R).

Dakhnovskii, Y., and H. Metiu, 1995, Phys. Rev. B 51, 4193.

De Dominicis, C., 1976, J. Phys. Colloques 37-C1, 247.

De Dominicis, C., and L. Peliti, 1978, Phys. Rev. B 18, 353.

Dietel, J., 2006, Phys. Rev. B 73, 125350.

Dietel, J., L. I. Glazman, F. W. J. Hekking, and F. von Oppen, 2005, Phys. Rev. B 71, 045329.

Dietrich, S., S. Byrnes, S. Vitkalov, D. V. Dmitriev, and A. A. Bykov, 2012a, Phys. Rev. B 85, 155307.

Dietrich, S., S. Byrnes, S. Vitkalov, A. V. Goran, and A. A. Bykov, 2012b, Phys. Rev. B 86, 075471.

Dietrich, S., S. Vitkalov, D. V. Dmitriev, and A. A. Bykov, 2012c, Phys. Rev. B 85, 115312.

Dingle, R., H. L. Störmer, A. C. Gossard, and W. Wiegmann, 1978, Appl. Phys. Lett. 33, 665.

Dmitriev, D. V., I. S. Strygin, A. A. Bykov, S. Dietrich, and S. A. Vitkalov, 2012, JETP Letters 95, 420.

Dmitriev, I. A., 2011, J. Phys.: Conf. Ser. 334, 012015.

Dmitriev, I. A., S. I. Dorozhkin, and A. D. Mirlin, 2009a, Phys. Rev. B 80, 125418.

Dmitriev, I. A., F. Evers, I. V. Gornyi, A. D. Mirlin, D. G. Polyakov, and P. Wölfle, 2008, Phys. Status Solidi (b) 245, 239.

Dmitriev, I. A., R. Gellmann, and M. G. Vavilov, 2010, Phys. Rev. B 82, 201311(R).

Dmitriev, I. A., M. Khodas, A. D. Mirlin, D. G. Polyakov, and M. G. Vavilov, 2009b, Phys. Rev. B 80, 165327.

Dmitriev, I. A., A. D. Mirlin, and D. G. Polyakov, 2003, Phys. Rev. Lett. 91, 226802.

Dmitriev, I. A., A. D. Mirlin, and D. G. Polyakov, 2004, Phys. Rev. B 70, 165305.

Dmitriev, I. A., A. D. Mirlin, and D. G. Polyakov, 2007a, Phys. Rev. B 75, 245320.

Dmitriev, I. A., A. D. Mirlin, and D. G. Polyakov, 2007b, Phys. Rev. Lett. 99, 206805.

Dmitriev, I. A., M. G. Vavilov, I. L. Aleiner, A. D. Mirlin, and D. G. Polyakov, 2005, Phys. Rev. B 71, 115316.

Dorozhkin, S. I., 2003, JETP Lett. 77, 577.

Dorozhkin, S. I., A. A. Bykov, I. V. Pechenezhskii, and A. K. Bakarov, 2007a, JETP Lett. 85, 576.

Dorozhkin, S. I., I. A. Dmitriev, and A. D. Mirlin, 2011a, Phys. Rev. B 84, 125448.

Dorozhkin, S. I., I. V. Pechenezhskiy, L. N. Pfeiffer, K. W. West, V. Umansky, K. von Klitzing, and J. H. Smet, 2009, Phys. Rev. Lett. 102, 036602.

Dorozhkin, S. I., L. Pfeiffer, K. West, K. von Klitzing, and J. H. Smet, 2011b, Nature Phys. 7, 336.

Dorozhkin, S. I., J. H. Smet, K. von Klitzing, L. N. Pfeiffer, and K. W. West, 2007b, JETP Lett. 86, 543.

Dorozhkin, S. I., J. H. Smet, V. Umansky, and K. von Klitzing, 2005, Phys. Rev. B 71, 201306(R).

Dreizin, Y. A., and A. M. Dykhne, 1973, Sov. Phys. JETP 36, 127.

Du, R. R., M. A. Zudov, C. L. Yang, Z. Q. Yuan, L. N. Pfeiffer, and K. W. West, 2004, Int. J. Mod. Phys. B 18, 3465.

Durst, A. C., 2006, Nature (London) 442, 752.

Durst, A. C., and S. M. Girvin, 2004, Science 304, 1752.

Durst, A. C., S. Sachdev, N. Read, and S. M. Girvin, 2003, Phys. Rev. Lett. 91, 086803.
Dyakonov, M. I., 1984, JETP Lett. 39, 185.

Dyakonov, M. I., and A. S. Furman, 1984, Sov. Phys. JETP 60, 1191.

Dykman, M. I., C. Fang-Yen, and M. J. Lea, 1997, Phys. Rev. B 55, 16249.

Dykman, M. I., M. J. Lea, P. Fozooni, and J. Frost, 1993, Phys. Rev. Lett. 70, 3975.

Dykman, M. I., and L. P. Pryadko, 2003, Phys. Rev. B 67, 235104.

Efetov, K. B., and V. G. Marikhin, 1989, Phys. Rev. B 40, 12126.

Efros, A. L., F. G. Pikus, and G. G. Samsonidze, 1990, Phys. Rev. B 41, 8295.

Eichhorn, R., P. Reimann, and P. Hänggi, 2002, Phys. Rev. E 66, 066132.

Elesin, V. F., 1969, Sov. Phys. JETP 28, 410.

Elesin, V. F., and E. A. Manykin, 1967, Sov. Phys. Solid State 8, 2891.

Evers, F., A. D. Mirlin, D. G. Polyakov, and P. Wölfle, 1999, Phys. Rev. B 60, 8951.

Fal'ko, V. I., and D. E. Khmel'nitskii, 1989, Sov. Phys. JETP 68, 1150 .

Fedorych, O. M., M. Potemski, S. A. Studenikin, J. A. Gupta, Z. R. Wasilewski, and I. A. Dmitriev, 2010, Phys. Rev. B 81, 201302(R).

Ferry, D., and S. Goodnick, 1997, Transport in Nanostructures (Cambridge University, Cambridge).

Finkler, I., B. I. Halperin, A. Auerbach, and A. Yacoby, 2006, J. Stat. Phys. 125, 1093.

Finkler, I. G., and B. I. Halperin, 2009, Phys. Rev. B 79, 085315.

Fitzgerald, R., 2003, Physics Today 56, 24.

Fogler, M. M., A. Y. Dobin, V. I. Perel, and B. I. Shklovskii, 1997, Phys. Rev. B 56, 6823.

Fogler, M. M., and B. I. Shklovskii, 1998, Phys. Rev. Lett. 80, 4749 .

Forster, D., D. R. Nelson, and M. J. Stephen, 1977, Phys. Rev. A 16, 732 .

Friedland, K.-J., R. Hey, H. Kostial, R. Klann, and K. Ploog, 1996, Phys. Rev. Lett. 77, 4616.

Galaktionov, E. A., A. K. Savchenko, and D. A. Ritchie, 2006, Phys. Status Solidi (c) 3, 304.

Geim, A. K., and K. S. Novoselov, 2007, Nature Mat. 6, 183.

Gerhardts, R. R., 1975, Z. Phys. B 21, 285.

Gerhardts, R. R., D. Weiss, and K. von Klitzing, 1989, Phys. Rev. Lett. 62, 1173.

Gershenzon, M. E., and M. I. Falei, 1986, JETP Lett. 44, 682 .

Gershenzon, M. E., and M. I. Falei, 1988, Sov. Phys. JETP 67, 389.

Girvin, S. M., M. Jonson, and P. A. Lee, 1982, Phys. Rev. B 26, 1651.

Gladun, A. D., and V. I. Ryzhii, 1970, Sov. Phys. JETP 30, 534.

Gold, A., 1989, Appl. Phys. Lett. 54, 2100.

Goran, A. V., A. A. Bykov, A. I. Toropov, and S. A. Vitkalov, 2009, Phys. Rev. B 80, 193305.

Gornyi, I. V., and A. D. Mirlin, 2003, Phys. Rev. Lett. 90, 076801.

Gornyi, I. V., and A. D. Mirlin, 2004, Phys. Rev. B 69, 045313.

Grégoire, G., and H. Chaté, 2004, Phys. Rev. Lett. 92, 025702.

Grimes, C. C., 1978, Surface Science 73, 379. 
Gunn, J. B., 1963, Solid State Commun. 1, 88.

Gurevich, V. L., and Y. A. Firsov, 1961, Sov. Phys. JETP 13, 137.

Gusev, G. M., S. Wiedmann, O. E. Raichev, A. K. Bakarov, and J. C. Portal, 2011, Phys. Rev. B 83, 041306(R).

Halperin, B. I., P. A. Lee, and N. Read, 1993, Phys. Rev. B 47, 7312 .

Hartmann, L., M. Grifoni, and P. Hänggi, 1997, Europhys. Lett. 38, 497.

Hatke, A. T., H.-S. Chiang, M. A. Zudov, L. N. Pfeiffer, and K. W. West, 2008a, Phys. Rev. Lett. 101, 246811.

Hatke, A. T., H.-S. Chiang, M. A. Zudov, L. N. Pfeiffer, and K. W. West, 2008b, Phys. Rev. B 77, 201304(R).

Hatke, A. T., H.-S. Chiang, M. A. Zudov, L. N. Pfeiffer, and K. W. West, 2010, Phys. Rev. B 82, 041304(R).

Hatke, A. T., M. Khodas, M. A. Zudov, L. N. Pfeiffer, and K. W. West, 2011a, Phys. Rev. B 84, 241302(R).

Hatke, A. T., M. A. Zudov, L. N. Pfeiffer, and K. W. West, 2009a, Phys. Rev. Lett. 102, 086808.

Hatke, A. T., M. A. Zudov, L. N. Pfeiffer, and K. W. West, 2009b, Phys. Rev. B 79, 161308(R).

Hatke, A. T., M. A. Zudov, L. N. Pfeiffer, and K. W. West, 2009c, Phys. Rev. Lett. 102, 066804.

Hatke, A. T., M. A. Zudov, L. N. Pfeiffer, and K. W. West, 2011b, Phys. Rev. B 83, 121301(R).

Hatke, A. T., M. A. Zudov, L. N. Pfeiffer, and K. W. West, 2011c, Phys. Rev. B 84, 241304(R).

Hatke, A. T., M. A. Zudov, L. N. Pfeiffer, and K. W. West, 2011d, Phys. Rev. B 83, 201301(R).

Hatke, A. T., M. A. Zudov, L. N. Pfeiffer, and K. W. West, 2011e, Phys. Rev. B 84, 121301(R).

Hatke, A. T., M. A. Zudov, L. N. Pfeiffer, and K. W. West, 2011f, Phys. Rev. B 83, 081301(R).

Hatke, A. T., M. A. Zudov, L. N. Pfeiffer, and K. W. West, 2012a, Phys. Rev. B 86, 081307(R).

Hatke, A. T., M. A. Zudov, L. N. Pfeiffer, and K. W. West, 2012b, Phys. Rev. B 85, 241305.

Hatke, A. T., M. A. Zudov, J. L. Reno, L. N. Pfeiffer, and K. W. West, 2012c, Phys. Rev. B 85, 081304(R).

Hatke, A. T., M. A. Zudov, J. D. Watson, and M. J. Manfra, 2012d, Phys. Rev. B 85, 121306.

Heiblum, M., E. E. Mendez, and F. Stern, 1984, Appl. Phys. Lett. 44, 1064.

Heinonen, O. (ed.), 1998, Composite Fermions: A Unified View of the Quantum Hall Regime (World Scientific, Singapore).

Herring, C., 1960, J. Appl. Phys. 31, 1939.

Hohenberg, P. C., and B. I. Halperin, 1977, Rev. Mod. Phys. 49, 435.

Houghton, A., J. R. Senna, and S. C. Ying, 1982, Phys. Rev. B 25, 2196.

Ignatov, A. A., E. Schomburg, J. Grenzer, K. F. Renk, and E. P. Dodin, 1995, Z. Phys. B 98, 187.

Ilani, S., A. Yacoby, D. Mahalu, and H. Shtrikman, 2000, Phys. Rev. Lett. 84, 3133.

Ilani, S., A. Yacoby, D. Mahalu, and H. Shtrikman, 2001, Science 292, 1354.

Imry, Y., and S.-K. Ma, 1975, Phys. Rev. Lett. 35, 1399.

Isichenko, M. B., 1992, Rev. Mod. Phys. 64, 961.

Isihara, A., and L. Smrčka, 1986, J. Phys. C 19, 6777.

Jain, J. K., 2007, Composite Fermions (Cambridge University, Cambridge).

Janssen, H.-K., 1976, Z. Physik B 23, 377.

Jauho, A. P., and K. Johnsen, 1996, Phys. Rev. Lett. 76,
4576.

Joas, C., J. Dietel, and F. von Oppen, 2005, Phys. Rev. B 72, 165323.

Joas, C., M. E. Raikh, and F. von Oppen, 2004, Phys. Rev. B 70, 235302.

Johnsen, K., and A.-P. Jauho, 1998, Phys. Rev. B 57, 8860.

Kalmanovitz, N. R., A. A. Bykov, S. A. Vitkalov, and A. I. Toropov, 2008, Phys. Rev. B 78, 085306.

Kashuba, A., 2006a, JETP Lett. 83, 293.

Kashuba, A., 2006b, Phys. Rev. B 73, 125340.

Kazarinov, R. F., and V. G. Skobov, 1963, Sov. Phys. JETP 17, 921.

Keay, B. J., S. Zeuner, J. S. J. Allen, K. D. Maranowski, A. C. Gossard, U. Bhattacharaya, and M. J. W. Rodwell, 1995, Phys. Rev. Lett. 75, 4102.

Keldysh, L. V., 1965, Sov. Phys. JETP 20, 1307.

Kennett, M. P., J. P. Robinson, N. R. Cooper, and V. I. Fal'ko, 2005, Phys. Rev. B 71, 195420.

Khodas, M., H.-S. Chiang, A. T. Hatke, M. A. Zudov, M. G. Vavilov, L. N. Pfeiffer, and K. W. West, 2010, Phys. Rev. Lett. 104, 206801.

Khodas, M., and M. G. Vavilov, 2008, Phys. Rev. B 78, 245319.

Khveshchenko, D. V., 1996, Phys. Rev. Lett. 77, 1817.

von Klitzing, K., 1986, Rev. Mod. Phys. 58, 519.

von Klitzing, K., G. Dorda, and M. Pepper, 1980, Phys. Rev. Lett. 45, 494.

Kogan, S. M., 1968, Sov. Phys. JETP 10, 1213.

Konstantinov, D., A. Chepelianskii, and K. Kono, 2012a, J. Phys. Soc. Jpn. 81, 093601.

Konstantinov, D., M. I. Dykman, M. J. Lea, Y. Monarkha, and K. Kono, 2009, Phys. Rev. Lett. 103, 096801.

Konstantinov, D., M. I. Dykman, M. J. Lea, Y. P. Monarkha, and K. Kono, 2012b, Phys. Rev. B 85, 155416.

Konstantinov, D., H. Isshiki, Y. Monarkha, H. Akimoto, K. Shirahama, and K. Kono, 2007, Phys. Rev. Lett. 98, 235302.

Konstantinov, D., H. Isshiki, Y. Monarkha, H. Akimoto, K. Shirahama, and K. Kono, 2008a, J. Phys. Soc. Jpn. 77, 034705.

Konstantinov, D., and K. Kono, 2009, Phys. Rev. Lett. 103, 266808.

Konstantinov, D., and K. Kono, 2010, Phys. Rev. Lett. 105, 226801.

Konstantinov, D., K. Kono, and Y. Monarkha, 2008b, Low Temp. Phys. 34, 377.

Koulakov, A. A., and M. E. Raikh, 2003, Phys. Rev. B 68, 115324.

Kroemer, H., 2001, Rev. Mod. Phys. 73, 783.

Kukushkin, I. V., M. Y. Akimov, J. H. Smet, S. A. Mikhailov, K. von Klitzing, I. L. Aleiner, and V. I. Falko, 2004, Phys. Rev. Lett. 92, 236803.

Kukushkin, I. V., S. A. Mikhailov, J. H. Smet, and K. von Klitzing, 2005, Appl. Phys. Lett. 86, 044101.

Laikhtman, B., and E. L. Altshuler, 1994, Ann. Phys. (N.Y.) 232, 332 .

Langenbuch, M., M. Suhrke, and U. Rössler, 2004, Phys. Rev. B 69, 125303.

Laughlin, R. B., 1999, Rev. Mod. Phys. 71, 863.

Lea, M. J., and M. I. Dykman, 1998, Physica B 249-251, 628.

Leadley, D. R., R. Fletcher, R. J. Nicholas, F. Tao, C. T. Foxon, and J. J. Harris, 1992, Phys. Rev. B 46, 12439.

Leadley, D. R., R. J. Nicholas, J. J. Harris, and C. T. Foxon, 
1989, Semicond. Sci. Technol. 4, 885.

Lee, D.-H., and J. M. Leinaas, 2004, Phys. Rev. B 69, 115336.

Lee, K., M. S. Shur, T. J. Drummond, and H. Morkoç, 1983, J. Appl. Phys. 54, 6432.

Lei, X. L., 2006, Phys. Rev. B 73, 235322.

Lei, X. L., 2008, Phys. Rev. B 77, 205309.

Lei, X. L., 2009, Phys. Rev. B 79, 115308.

Lei, X. L., 2010, Mater. Sci. Eng., R 70, 126.

Lei, X. L., and S. Y. Liu, 2003, Phys. Rev. Lett. 91, 226805.

Lei, X. L., and S. Y. Liu, 2006a, Appl. Phys. Lett. 89, 182117.

Lei, X. L., and S. Y. Liu, 2006b, Appl. Phys. Lett. 88, 212109.

Levinson, Y., O. Entin-Wohlman, A. D. Mirlin, and P. Wölfle, 1998, Phys. Rev. B 58, 7113.

Li, L., Y. Y. Proskuryakov, A. K. Savchenko, E. H. Linfield, and D. A. Ritchie, 2003, Phys. Rev. Lett. 90, 076802.

Liao, P. F., A. M. Glass, and L. M. Humphrey, 1980, Phys. Rev. B 22, 2276.

Lifshitz, I. M., M. Y. Azbel, and M. I. Kaganov, 1973, Electron Theory of Metals (Consultants Bureau, New York).

Lyapilin, I. I., and A. E. Patrakov, 2004, Low Temp. Phys. $\mathbf{3 0}, 834$.

Lyapilin, I. I., and A. E. Patrakov, 2006, Phys. Met. Metallogr. 102, 560.

Mamani, N. C., G. M. Gusev, T. E. Lamas, A. K. Bakarov, and O. E. Raichev, 2008, Phys. Rev. B 77, 205327.

Mamani, N. C., G. M. Gusev, O. E. Raichev, T. E. Lamas, and A. K. Bakarov, 2009a, Phys. Rev. B 80, 075308.

Mamani, N. C., G. M. Gusev, E. C. F. da Silva, O. E. Raichev, A. A. Quivy, and A. K. Bakarov, 2009b, Phys. Rev. B 80, 085304 .

Mani, R., 2004a, Physica E 22, 1.

Mani, R. G., 2004b, Appl. Phys. Lett. 85, 4962.

Mani, R. G., 2005, Phys. Rev. B 72, 075327.

Mani, R. G., 2007a, Solid State Commun. 144, 409.

Mani, R. G., 2007b, Appl. Phys. Lett. 91, 132103.

Mani, R. G., 2008, Appl. Phys. Lett. 92, 102107.

Mani, R. G., C. Gerl, S. Schmult, W. Wegscheider, and V. Umansky, 2010, Phys. Rev. B 81, 125320.

Mani, R. G., W. B. Johnson, V. Umansky, V. Narayanamurti, and K. Ploog, 2009, Phys. Rev. B 79, 205320.

Mani, R. G., V. Narayanamurti, K. von Klitzing, J. H. Smet, W. B. Johnson, and V. Umansky, 2004a, Phys. Rev. B 69, 161306(R).

Mani, R. G., V. Narayanamurti, K. von Klitzing, J. H. Smet, W. B. Johnson, and V. Umansky, 2004b, Phys. Rev. B 70, 155310 .

Mani, R. G., A. N. Ramanayaka, and W. Wegscheider, 2011, Phys. Rev. B 84, 085308.

Mani, R. G., J. H. Smet, K. von Klitzing, V. Narayanamurti, W. B. Johnson, and V. Umansky, 2002, Nature (London) 420, 646 .

Mani, R. G., J. H. Smet, K. von Klitzing, V. Narayanamurti, W. B. Johnson, and V. Umansky, 2004c, Phys. Rev. Lett. 92, 146801.

Mani, R. G., J. H. Smet, K. von Klitzing, V. Narayanamurti, W. B. Johnson, and V. Umansky, 2004d, Phys. Rev. B 69, 193304.

Markus, Y., U. Meirav, H. Shtrikman, and B. Laikhtman, 1994, Semicond. Sci. Technol. 9, 1297.

Martin, G. W., D. L. Maslov, and M. Y. Reizer, 2003, Phys. Rev. B 68, 241309.

Martin, P. C., E. D. Siggia, and H. A. Rose, 1973, Phys. Rev. A 8, 423.

McCann, E., and V. I. Fal'ko, 2006, Phys. Rev. Lett. 96,
086805 .

Mikhailov, S. A., 2004, Phys. Rev. B 70, 165311.

Mikhailov, S. A., 2011, Phys. Rev. B 83, 155303.

Mikhailov, S. A., and N. A. Savostianova, 2006, Phys. Rev. B 74, 045325.

Mirlin, A. D., E. Altshuler, and P. Wölfle, 1996, Ann. Phys. (Leipzig) 508, 281.

Mirlin, A. D., D. G. Polyakov, F. Evers, and P. Wölfle, 2001, Phys. Rev. Lett. 87, 126805.

Mirlin, A. D., D. G. Polyakov, and P. Wölfle, 1998, Phys. Rev. Lett. 80, 2429.

Mirlin, A. D., J. Wilke, F. Evers, D. G. Polyakov, and P. Wölfle, 1999, Phys. Rev. Lett. 83, 2801.

Mirlin, A. D., and P. Wölfle, 1998, Phys. Rev. B 58, 12986.

Mkhitaryan, V. V., and M. E. Raikh, 2011, Phys. Rev. B 83, 045406 .

Monarkha, Y., and K. Kono, 2004, Two-Dimensional Coulomb Liquids and Solids (Springer Verlag).

Monarkha, Y. P., 2011a, Low Temp. Phys. 37, 90.

Monarkha, Y. P., 2011b, Low Temp. Phys. 37, 655.

Monarkha, Y. P., 2012, Low Temp. Phys. 38, 451.

Mueller, T., F. Xia, and P. Avouris, 2010, Nature Phot. 4, 297.

Murzin, S. S., 1984, JETP Lett. 39, 695.

Nachtwei, G., 1999, Physica E 4, 79.

Nachtwei, G., F. Gouider, C. Stellmach, G. Vasile, Y. B. Vasilyev, G. Hein, and R. R. Gerhardts, 2008, Phys. Rev. B 78, 174305 .

Neugebauer, P., M. Orlita, C. Faugeras, A.-L. Barra, and M. Potemski, 2009, Phys. Rev. Lett. 103, 136403.

Novoselov, K. S., A. K. Geim, S. V. Morozov, D. Jiang, M. I. Katsnelson, I. V. Grigorieva, S. V. Dubonos, and A. A. Firsov, 2005, Nature (London) 438, 197.

Novoselov, K. S., A. K. Geim, S. V. Morozov, D. Jiang, Y. Zhang, S. V. Dubonos, I. V. Grigorieva, and A. A. Firsov, 2004, Science 306, 666.

Novoselov, K. S., Z. Jiang, Y. Zhang, S. V. Morozov, H. L. Stormer, U. Zeitler, J. C. Maan, G. S. Boebinger, P. Kim, and A. K. Geim, 2007, Science 315, 1379.

Novoselov, K. S., E. McCann, S. V. Morozov, V. I. Fal'ko, M. I. Katsnelson, U. Zeitler, D. Jiang, F. Schedin, and A. K. Geim, 2006, Nature Phys. 2, 177.

Olshanetsky, E. B., V. Renard, Z. D. Kvon, J. C. Portal, N. J. Woods, J. Zhang, and J. J. Harris, 2005, Europhys. Lett. $\mathbf{7 1}, 665$.

Orlita, M., and M. Potemski, 2010, Semicond. Sci. Technol. 25, 063001.

Paalanen, M. A., D. C. Tsui, and J. C. M. Hwang, 1983, Phys. Rev. Lett. 51, 2226.

Park, K., 2004, Phys. Rev. B 69, 201301(R).

Pavlovich, V. V., and E. M. Epshtein, 1976, Sov. Phys. Semicond. 10, 1196.

Pechenezhskii, I. V., S. I. Dorozhkin, and I. A. Dmitriev, 2007, JETP Lett. 85, 86.

Pfeiffer, L., and K. W. West, 2003, Physica E 20, 57.

Pfeiffer, L., K. W. West, H. L. Stormer, and K. W. Baldwin, 1989, Appl. Phys. Lett. 55, 1888.

Pippard, A. B., 1989, Magnetoresistance in Metals (Cambridge University, Cambridge).

Polyakov, D. G., 1986, Sov. Phys. JETP 63, 317.

Polyakov, D. G., F. Evers, and I. V. Gornyi, 2002, Phys. Rev. B 65, 125326.

Polyakov, D. G., F. Evers, A. D. Mirlin, and P. Wölfle, 2001, Phys. Rev. B 64, 205306. 
Polyanovsky, V., 1988, Sov. Phys. Semicond. 22, 1408.

Pozhela, J. K., 1981, Plasma and current instabilities in semiconductors (Pergamon, Oxford).

Prange, R. E., and T.-W. Nee, 1968, Phys. Rev. 168, 779.

Pruisken, A. M. M., 1990, in The Quantum Hall Effect, edited by R. E. Prange and S. M. Girvin (Springer, Berlin).

Raichev, O. E., 2008, Phys. Rev. B 78, 125304.

Raichev, O. E., 2009, Phys. Rev. B 80, 075318.

Raichev, O. E., 2010a, Phys. Rev. B 81, 195301.

Raichev, O. E., 2010b, Phys. Rev. B 81, 165319.

Raikh, M. E., and T. V. Shahbazyan, 1993, Phys. Rev. B 47, 1522.

Raikh, M. E., and T. V. Shahbazyan, 1994, Phys. Rev. B 49, 5531.

Ridley, B. K., 1963, Proc. Phys. Soc. 82, 954.

Robinson, J. P., M. P. Kennett, N. R. Cooper, and V. I. Fal'ko, 2004, Phys. Rev. Lett. 93, 036804.

Romero, N., S. McHugh, M. P. Sarachik, S. A. Vitkalov, and A. A. Bykov, 2008, Phys. Rev. B 78, 153311.

Rowe, A. C. H., J. Nehls, R. A. Stradling, and R. S. Ferguson, 2001, Phys. Rev. B 63, 201307(R).

Ryzhii, V., A. Chaplik, and R. Suris, 2004, JETP Lett. 80, 363.

Ryzhii, V., and R. Suris, 2003, J. Phys.: Condens. Matter 15, 6855 .

Ryzhii, V. I., 1970, Sov. Phys. Solid State 11, 2078.

Ryzhii, V. I., R. A. Suris, and B. S. Shchamkhalova, 1986, Sov. Phys. Semicond. 20, 1299.

Sadowski, M. L., G. Martinez, M. Potemski, C. Berger, and W. A. de Heer, 2006, Phys. Rev. Lett. 97, 266405.

Saku, T., Y. Horikoshi, and Y. Tokura, 1996, Jpn. J. Appl. Phys. 35, 34.

Sander, T. H., S. N. Holmes, J. J. Harris, D. K. Maude, and J. C. Portal, 1998, Phys. Rev. B 58, 13856.

Schöll, E., 2001, Nonlinear spatio-temporal dynamics and chaos in semiconductors (Cambridge University, Cambridge).

Sedrakyan, T. A., and M. E. Raikh, 2008, Phys. Rev. Lett. 100, 086808.

Seely, J. F., 1974, Phys. Rev. A 10, 1863.

Shayegan, M., V. J. Goldman, C. Jiang, T. Sajoto, and M. Santos, 1988, Appl. Phys. Lett. 52, 1086.

Shi, J., and X. C. Xie, 2003, Phys. Rev. Lett. 91, 086801.

Shikler, R., M. Heiblum, and V. Umansky, 1997, Phys. Rev. B 55, 15427.

Shoenberg, D., 1984, Magnetic Oscillations in Metals (Cambridge University, Cambridge).

Smet, J. H., 1997, unpublished .

Smet, J. H., 1998, in Composite Fermions, edited by O. Heinonen (World Scientific, Singapore).

Smet, J. H., B. Gorshunov, C. Jiang, L. Pfeiffer, K. West, V. Umansky, M. Dressel, R. Meisels, F. Kuchar, and K. von Klitzing, 2005, Phys. Rev. Lett. 95, 116804.

Sollner, T. C. L. G., W. D. Goodhue, P. E. Tannenwald, C. D. Parker, and D. D. Peck, 1983, Appl. Phys. Lett. 43, 588.

Stellmach, C., G. Vasile, A. Hirsch, R. Bonk, Y. B. Vasilyev, G. Hein, C. R. Becker, and G. Nachtwei, 2007, Phys. Rev. B 76, 035341.

Stern, F., 1983, Appl. Phys. Lett. 43, 974.

Stone, K., C. L. Yang, Z. Q. Yuan, R. R. Du, L. N. Pfeiffer, and K. W. West, 2007, Phys. Rev. B 76, 153306.

Stormer, H. L., 1999, Rev. Mod. Phys. 71, 875.

Studenikin, S. A., G. Granger, A. Kam, A. S. Sachrajda, Z. R. Wasilewski, and P. J. Poole, 2012, Phys. Rev. B 86, 115309.
Studenikin, S. A., M. Potemski, P. T. Coleridge, A. S. Sachrajda, and Z. R. Wasilewski, 2004, Solid State Commun. 129, 341.

Studenikin, S. A., M. Potemski, A. Sachrajda, M. Hilke, L. N. Pfeiffer, and K. W. West, 2005, Phys. Rev. B 71, 245313.

Studenikin, S. A., A. S. Sachrajda, J. A. Gupta, Z. R. Wasilewski, O. M. Fedorych, M. Byszewski, D. K. Maude, M. Potemski, M. Hilke, K. W. West, and L. N. Pfeiffer, 2007, Phys. Rev. B 76, 165321.

Tan, Z., C. Tan, L. Ma, G. T. Liu, L. Lu, and C. L. Yang, 2011, Phys. Rev. B 84, 115429.

Tokura, Y., T. Saku, S. Tarucha, and Y. Horikoshi, 1992, Phys. Rev. B 46, 15558(R).

Toner, J., and Y. Tu, 1995, Phys. Rev. Lett. 75, 4326.

Toner, J., and Y. Tu, 1998, Phys. Rev. E 58, 4828.

Torres, M., and A. Kunold, 2005, Phys. Rev. B 71, 115313.

Torres, M., and A. Kunold, 2006, J. Phys.: Condens. Matter 18, 4029.

Tsu, R., and L. Esaki, 1973, Appl. Phys. Lett. 22, 562.

Tsui, D. C., 1999, Rev. Mod. Phys. 71, 891.

Tsui, D. C., T. Englert, A. Y. Cho, and A. C. Gossard, 1980, Phys. Rev. Lett. 44, 341.

Tsui, D. C., H. L. Stormer, and A. C. Gossard, 1982, Phys. Rev. B 25, 1405(R).

Tung, L.-C., C. L. Yang, D. Smirnov, L. N. Pfeiffer, K. W. West, R. R. Du, and Y.-J. Wang, 2009, Solid State Commun. 149, 1531.

Umansky, V., M. Heiblum, Y. Levinson, J. Smet, J. Nübler, and M. Dolev, 2009, J. Cryst. Growth 311, 1658.

Umansky, V., R. de Picciotto, and M. Heiblum, 1997, Appl. Phys. Lett. 71, 683.

Vavilov, M. G., and I. L. Aleiner, 2004, Phys. Rev. B 69, 035303.

Vavilov, M. G., I. L. Aleiner, and L. I. Glazman, 2007, Phys. Rev. B 76, 115331.

Vavilov, M. G., I. A. Dmitriev, I. L. Aleiner, A. D. Mirlin, and D. G. Polyakov, 2004, Phys. Rev. B 70, 161306(R).

Vicsek, T., A. Czirók, E. Ben-Jacob, I. Cohen, and O. Shochet, 1995, Phys. Rev. Lett. 75, 1226.

Vitkalov, S., 2009, Int. J. Mod. Phys. B 23, 4727.

Volkov, A. F., and S. M. Kogan, 1967, Sov. Phys. JETP 25, 1095.

Volkov, A. F., and S. M. Kogan, 1969, Sov. Phys. Usp. 11, 881.

Volkov, A. F., and V. V. Pavlovskii, 2004, Phys. Rev. B 69, 125305.

Volkov, V. A., and E. E. Takhtamirov, 2007, JETP 104, 602.

Walukiewicz, W., H. E. Ruda, J. Lagowski, and H. C. Gatos, 1984, Phys. Rev. B 30, 4571.

Weiss, D., K. von Klitzing, K. Ploog, and G. Weimann, 1989, Europhys. Lett. 8, 179.

Weiss, D., M. L. Roukes, A. Menschig, P. Grambow, K. von Klitzing, and G. Weimann, 1991, Phys. Rev. Lett. 66, 2790.

Wiedmann, S., G. M. Gusev, O. E. Raichev, A. K. Bakarov, and J. C. Portal, 2009a, Phys. Rev. B 80, 035317.

Wiedmann, S., G. M. Gusev, O. E. Raichev, A. K. Bakarov, and J. C. Portal, 2010a, Phys. Rev. B 82, 165333.

Wiedmann, S., G. M. Gusev, O. E. Raichev, A. K. Bakarov, and J. C. Portal, 2010b, Phys. Rev. B 81, 085311.

Wiedmann, S., G. M. Gusev, O. E. Raichev, A. K. Bakarov, and J. C. Portal, 2010c, Phys. Rev. Lett. 105, 026804.

Wiedmann, S., G. M. Gusev, O. E. Raichev, A. K. Bakarov, and J. C. Portal, 2011a, Phys. Rev. B 84, 165303.

Wiedmann, S., G. M. Gusev, O. E. Raichev, S. Krämer, A. K. 
Bakarov, and J. C. Portal, 2011b, Phys. Rev. B 83, 195317.

Wiedmann, S., G. M. Gusev, O. E. Raichev, T. E. Lamas, A. K. Bakarov, and J. C. Portal, 2008, Phys. Rev. B 78, 121301.

Wiedmann, S., N. C. Mamani, G. M. Gusev, O. E. Raichev, A. K. Bakarov, and J. C. Portal, 2009b, Phys. Rev. B 80, 245306.

Willett, R. L., J. W. P. Hsu, D. Natelson, K. W. West, and L. N. Pfeiffer, 2001, Phys. Rev. Lett. 87, 126803.

Willett, R. L., L. N. Pfeiffer, and K. W. West, 2004, Phys. Rev. Lett. 93, 026804.

Wirthmann, A., B. D. McCombe, D. Heitmann, S. Holland, K.-J. Friedland, and C.-M. Hu, 2007, Phys. Rev. B 76, 195315.

Yacoby, Y., 1968, Phys. Rev. 169, 610.

Yang, C. L., R. R. Du, L. N. Pfeiffer, and K. W. West, 2006, Phys. Rev. B 74, 045315.

Yang, C. L., J. Zhang, R. R. Du, J. A. Simmons, and J. L. Reno, 2002a, Phys. Rev. Lett. 89, 076801.

Yang, C. L., M. A. Zudov, T. A. Knuuttila, R. R. Du, L. N. Pfeiffer, and K. W. West, 2003, Phys. Rev. Lett. 91, 096803.

Yang, C. L., M. A. Zudov, J. Zhang, R. R. Du, J. A. Simmons, and J. L. Reno, 2002b, Physica E 12, 443.

Ye, P. D., L. W. Engel, D. C. Tsui, J. A. Simmons, J. R. Wendt, G. A. Vawter, and J. L. Reno, 2001, Appl. Phys. Lett. 79, 2193.

Yuan, Z. Q., C. L. Yang, R. R. Du, L. N. Pfeiffer, and K. W. West, 2006, Phys. Rev. B 74, 075313.

Zakharov, A. L., 1960, Sov. Phys. JETP 11, 478.

Zaremba, E., 1992, Phys. Rev. B 45, 14143.

Zeldovich, Y. B., 1967, Sov. Phys. JETP 24, 1006.

Zhang, C., and R. R. Gerhardts, 1990, Phys. Rev. B 41, 12850.

Zhang, J., S. K. Lyo, R. R. Du, J. A. Simmons, and J. L.
Reno, 2004, Phys. Rev. Lett. 92, 156802.

Zhang, J. Q., S. Vitkalov, and A. A. Bykov, 2009, Phys. Rev. B 80, 045310.

Zhang, J. Q., S. Vitkalov, A. A. Bykov, A. K. Kalagin, and A. K. Bakarov, 2007a, Phys. Rev. B 75, 081305(R).

Zhang, W., H.-S. Chiang, M. A. Zudov, L. N. Pfeiffer, and K. W. West, 2007b, Phys. Rev. B 75, 041304(R).

Zhang, W., M. A. Zudov, L. N. Pfeiffer, and K. W. West, 2007c, Phys. Rev. Lett. 98, 106804.

Zhang, W., M. A. Zudov, L. N. Pfeiffer, and K. W. West, 2008a, Physica E 40, 982.

Zhang, W., M. A. Zudov, L. N. Pfeiffer, and K. W. West, 2008b, Phys. Rev. Lett. 100, 036805.

Zhang, Y., Y.-W. Tan, H. L. Stormer, and P. Kim, 2005, Nature (London) 438, 201.

Zudov, M. A., 2004, Phys. Rev. B 69, 041304(R).

Zudov, M. A., H.-S. Chiang, A. T. Hatke, W. Zhang, L. N. Pfeiffer, and K. W. West, 2009, Int. J. Mod. Phys. B 23, 2684.

Zudov, M. A., R. R. Du, L. N. Pfeiffer, and K. W. West, 2003, Phys. Rev. Lett. 90, 046807.

Zudov, M. A., R. R. Du, L. N. Pfeiffer, and K. W. West, 2006a, Phys. Rev. Lett. 96, 236804.

Zudov, M. A., R. R. Du, L. N. Pfeiffer, and K. W. West, 2006b, Phys. Rev. B 73, 041303(R).

Zudov, M. A., R. R. Du, J. A. Simmons, and J. L. Reno, 1997, arXiv:cond-mat/9711149v1 .

Zudov, M. A., R. R. Du, J. A. Simmons, and J. L. Reno, 2001a, Phys. Rev. B 64, 201311(R).

Zudov, M. A., A. T. Hatke, H.-S. Chiang, L. N. Pfeiffer, K. W. West, and J. L. Reno, 2011, J. Phys.: Conf. Ser. 334, 012007.

Zudov, M. A., I. V. Ponomarev, A. L. Efros, R. R. Du, J. A. Simmons, and J. L. Reno, 2001b, Phys. Rev. Lett. 86, 3614. 